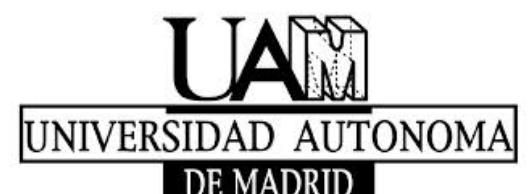

\title{
Applications of Holography to Condensed Matter Physics
}

\author{
Memoria de Tesis Doctoral realizada por

\section{Luis Fernando Melgar del Pozo} \\ presentada ante el Departamento de Física Teórica \\ de la Universidad Autónoma de Madrid \\ para la obtención del título de Doctor en Física Teórica.
}

Tesis Doctoral dirigida por

Karl Landsteiner del I.F.T.

de la Universidad Autónoma de Madrid

Madrid, Mayo de 2014. 
Dedicado a Mi Familia 


\begin{abstract}
We carry out a computation of chiral conductivities at strong coupling using holographic methods in Part II. To that end we implement chiral and mixed anomalies in the bulk by the use of suitable Chern-Simons terms. Then we study the cutoff flow of anomalous conductivities by computing them at a hypersurface placed at certain radial position in spacetime, which models the energy cutoff.

Furthermore, we construct and analyze the holographic version of a two-component superfluid. A two-component superfluid has $U(2) \rightarrow U(1)$ as the pattern of spontaneously broken symmetries and presents typically non-relativistic Goldstone bosons in its spectrum of excitations (the so-called type II Goldstone modes). We compute conductivities and dispersion relations for the hydrodynamic modes of the theory, as well as the gap of the so-called "massive Goldstone", a massive excitation that has been predicted to have a universal gap. Moreover, motivated by the presence of type II Goldstone bosons, we perform an analysis of the well-known Landau Criterion in the framework of our holographic models, showing that it holds. In addition, we construct a $s+$ p-superfluid, which features coexisting scalar and vector condensates. We also study its phase diagram after switching on two chemical potentials.

Finally, we compute anomalous conductivities for $U(1)$ holographic superfluids. Along the way we also point out that there is charge density present whenever the superfluid velocity is aligned with an external magnetic field. This effect absent if the theory is non-anomalous. The outcome of our calculation is that all chiral conductivities seem to approach universal values at zero temperature, even though they are not universal at finite temperature. Such a universal value depends only on the interplay between broken symmetries and the anomaly.
\end{abstract}

\title{
Resumen
}

Llevamos a cabo un cálculo de conductividades quirales en acoplamiento fuerte utilizando métodos holográficos en la Parte II. Para ello implementamos las anomalías quiral y mixta en el bulk utilizando los términos de Chern-Simons adecuados. A continuación se estudia el flujo de las conductividades anómalas en función de una escala de energía, calculándolas en una hipersuperficie colocada a cierto valor de la coordenada radial en el espacio-tiempo, que modela dicho cutoff de energía.

Además, construimos y analizamos la versión holográfica de un superfluido de dos componentes. Un superfluido de dos componentes tiene $U(2) \rightarrow U(1)$ como patrón de simetrías espontáneamente rotas y presenta bosones de Goldstone típicamente no relativistas en su espectro de excitaciones (los llamados modos de Goldstone de tipo II). Calculamos conductividades y relaciones de dispersión para los modos de hidrodinámicos de la teoría, así como la masa del llamado "Goldstone masivo", una excitación que se ha predicho que posee una masa universal. Por otra parte, motivado por la presencia de bosones de Goldstone de tipo II, llevamos a cabo un estudio del conocido Criterio de Landau en el marco de nuestros modelos holográficos, demostrando que se cumple. Además, se construye un superfluido $\mathrm{s}+\mathrm{p}$, que se caracteriza por tener condensados escalar y vectorial coexistentes. También estudiamos el diagrama de fase tras encender dos potenciales químicos.

Por último, calculamos conductividades anómalas para superfluidos holográficas $U(1)$. En el proceso también enfatizamos la existencia de una densidad de carga presente siempre que la supervelocidad esté alineada con un campo magnético externo. Este efecto está ausente si la teoría no es anómala. El resultado de nuestro cálculo se resume en que todas 
las conductividades quirales parecen aproximarse a valores universales a temperatura cero, a pesar de que no son universales a temperatura finita. Dicho valor universal sólo depende de la interacción entre las simetrías rotas y la(s) anomalía(s). 


\section{Acknowledgements}

Me gustaría empezar agradeciendo a Karl por enseñarme a investigar y por estar siempre disponible para hablar de física. También por frenarme cuando me iba demasiado lejos y espolearme cuando me paraba. ¡Muchas gracias por todo Karl! También agradecer a todos aquellos colaboradores que he tenido. Gracias a Fran (mi hermano cientifico mayor), Eugenio, Daniel, Nacho, Irene (mi jóven abuela científica) y sobre todo a Amadeo por su gran trabajo. Dar las gracias también a la gente con la que he hablado de física a menudo, aunque no haya publicado con ellos en ningún artículo (aún), en especial a Ioannis Papadimitriou y a Carlos Hoyos. También a los profesores del Máster en Física Teórica, en concreto a Carlos Pena, José Barbón y Margarita García. Agradezco mucho la confianza y el trato recibido por Jerome P. Gauntlett, Christiana Pantelidou y Graziela De Nadai Sowrey en mi visita científica a Imperial College London.

También quiero agradecer al personal de la quinta planta porque sin ellas realizar un doctorado sería una misión practicamente imposible. Isabel, Mónica, Roxanna, Susana, Chabely y Mónica, muchas gracias por haber estado siempre ahi peleando por nosotros.

En un plano más personal, quiero empezar agradeciendo a los amigos y compañeros del IFT. Gracias a David, Rodrigo, Antonio (de Antonio), Mikel, Jose Daniel, Amadeo, Diego, Pablo, Javi, Carlos (y a alguno más del que seguro me olvido) por todos los buenos ratos y las conversaciones. Gracias de nuevo a las secretarias y a Charo por las charlas en la comida que tan fácilmente me permitían desconectar de la física por un rato.

Más allá del IFT, me gustaría mencionar a Ignacio Guerra y sobre todo a Elia porque sin ella seguramente nunca habría empezado la carrera de física (ni ninguna otra cosa). Allá donde estéis, ¡Gracias!.

Por último y más importante, quiero dar las gracias a mi familia: mis padres Luis y Delmi y mi hermana Sara. Si cada día trato de mejorar es exclusivamente para intentar que ellos se sientan orgullosos y devolverles una infima parte de todo lo que me han dado. Todo lo que esta tesis tiene de mío, es suyo. Excepto las palabras. 
"Words, Words, Words"

Hamlet

Act 2, Scene 2 


\section{Contents}

$\begin{array}{ll}\text { Abstract } & \text { iii }\end{array}$

Acknowledgements $\quad$ v

I Introduction 5

\begin{tabular}{|lll}
1 & Remarks on gauge/gravity duality and the Holographic Dictionary & 6
\end{tabular}

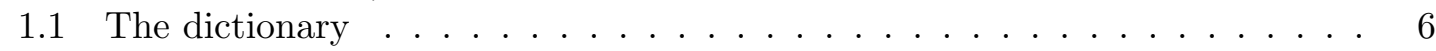

1.2 Global currents and chemical potential in holography . . . . . . . . . . . 8

\begin{tabular}{|lll}
\hline & Holographic approximations to out-of-equilibrium physics & 11
\end{tabular}

2.1 Global Equilibrium . . . . . . . . . . . . . . . . . . . 11

2.1.1 Global Equilibrium state as a geometric object in $A d S / C F T$. . . . 11

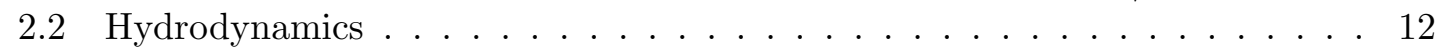

2.2 .1 The frame choice in non-ideal Hydrodynamics. . . . . . . . . . . . . 14

$2.2 .2 \quad$ Hydrodynamics and Holography $\ldots \ldots \ldots \ldots$. . . . . . . . 15

2.3 Linear response theory $\ldots \ldots \ldots \ldots \ldots \ldots$

2.3 .1 Linear Response Theory in Holography . . . . . . . . . . . . . . 18

2.4 Hydrodynamic Linear response $\ldots \ldots \ldots$. . . . . . . . . . . . . . . 21

$\begin{array}{lll}3 & \text { Anomalies and Chiral transport phenomena at weak coupling } & 24\end{array}$

3.1 Chemical potential for anomalous symmetries . . . . . . . . . . . . 27

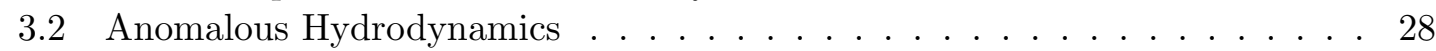

3.2.1 Transformations under Charge Conjugation(C) and Parity $(\mathrm{P})$. . . . 29

3.2 .2 Constraints from positivity of the entropy current . . . . . . . . 30

3.3 Anomalies and Kubo formulae . . . . . . . . . . . . . . . . . . 31

3.3 .1 Anomalous conductivities at weak coupling from Kubo Formulae . . 33

\begin{tabular}{|lll}
\hline & Relativistic Superfluids & 35
\end{tabular}

4.1 A model for a superfluid . . . . . . . . . . . . . . . . . . . 36

4.2 Spectrum of excitations: Sound . . . . . . . . . . . . . . . . 38

4.3 Generalizations $\ldots \ldots \ldots \ldots \ldots$

4.4 Goldstone Theorem and type II NG bosons . . . . . . . . . . . . . . . . . . 41

$4.4 .1 \quad$ A field theoretical model with type II Goldstone boson . . . . . . . . 42

4.5 Superfluid (anomalous) hydrodynamics . . . . . . . . . . . . . . 43 
II Anomalous Transport in Holography 45

$\begin{array}{lll}5 & \text { Holographic Mixed Gauge-Gravitational anomaly and transport } & 46\end{array}$

5.1 Holographic Model . . . . . . . . . . . . . . . . . 47

5.2 Kubo formulas, anomalies and Chiral Vortical Conductivity . . . . . . . . . 49

$6 \quad$ Cutoff Flow of Anomalous Transport Coefficients in Holography 53

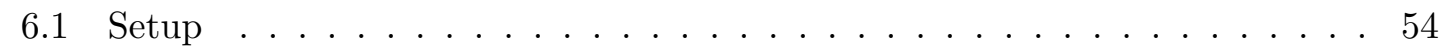

6.2 Flow of anomalous conductivities . . . . . . . . . . . . . 55

6.2 .1 Vector and Axial symmetries . . . . . . . . . . . . 55

$6.2 .2 \quad$ Inclusion of metric perturbations . . . . . . . . . . . . . . . . 59

$6.2 .3 \quad$ Application to the anomalous conductivities . . . . . . . . . . . . 59

6.3 Flow of the transport coefficients as two point functions . . . . . . . . . . 62

6.3 .1 Compatibility with the flow equations . . . . . . . . . . . . 63

6.4 Gravitational Anomaly . . . . . . . . . . . . . . . . . . . . . . . 64

6.4 .1 The Model . . . . . . . . . . . . . . . . . . . . 64

6.4 .2 Contribution of the Gravitational Anomaly . . . . . . . . . . . 65

\begin{tabular}{lll}
\hline & Discussion and Conclusion & 69
\end{tabular}

7.1 Holographic Renormalization . . . . . . . . . . . . . . . . . . . . 71

7.1 .1 Technical details on Holographic Renormalization . . . . . . . . . . . 71

7.1.2 Holographic Renormalization of the Mixed Anomaly . . . . . . . . . 75

7.2 Codazzi form of Equations of Motion . . . . . . . . . . . . . 76

7.3 Solutions at zero frequency and normalized at finite cutoff $u_{c} \ldots \ldots$. . . 77

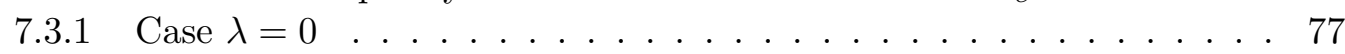

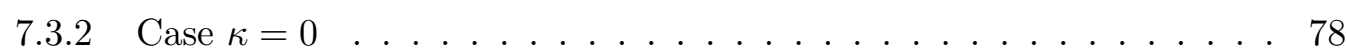

7.4 Equations of motion for the shear sector $\ldots \ldots \ldots \ldots$. . . . . . . 79

\begin{tabular}{lll}
\hline III & Exotic Holographic Superfluids & 80
\end{tabular}

$\begin{array}{lll}8 & \text { Non-superconducting BECs and Type-II Nambu-Goldstone Bosons } & 83\end{array}$

8.1 The ungauged model . . . . . . . . . . . . . . . . . 84

8.2 The gauged model $\ldots \ldots \ldots \ldots \ldots \ldots \ldots$

$8.2 .1 \quad$ Charge Density in the broken phase . . . . . . . . . . . . . . 95

$8.2 .2 \quad$ Fluctuations of the gauged model . . . . . . . . . . . . . . . . . 96

8.2 .3 Conductivities $\ldots \ldots \ldots \ldots$. . . . . . . . . . . . . . 99

$8.2 .4 \quad$ Conductivities in the $(0)-(3)$ sector . . . . . . . . . . . . . . . . 99

8.2 .5 Conductivities in the $(1)-(2)$ sector . . . . . . . . . . . . . . . 100

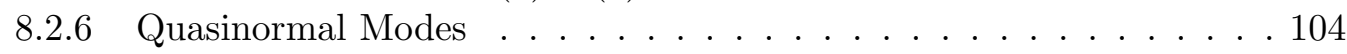

9 The Landau criterion in Holography 109

9.0 .7 The $U(2)$ superfluid with superflow . . . . . . . . . . . . . . . . . 111

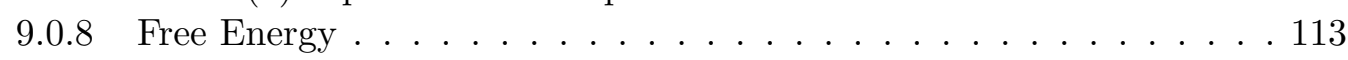

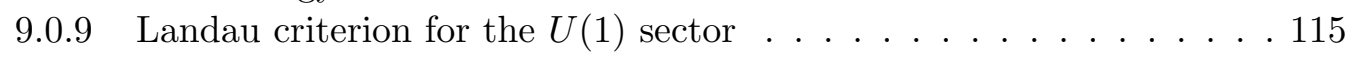

9.0 .10 Landau criterion for holographic Type II Goldstone bosons . . . . . 120

\begin{tabular}{lr}
\hline 0 Holographic s+p Superfluids & 123
\end{tabular}

10.1 The holographic two-component superfluid . . . . . . . . . . . . . . . . . . 124

10.2 The s+p-wave holographic superconductor . . . . . . . . . . . . . . . . 125

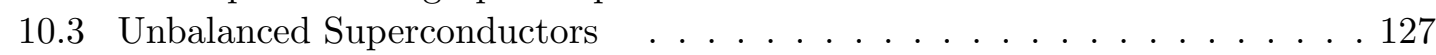


11 Discussion and Outlook 130

11.1 Fluctuation equations in the $(0)-(3)$ sector $\ldots \ldots \ldots . \ldots . \ldots . \ldots 135$

11.2 Fluctuation equations in the $(1)-(2)$ sector . . . . . . . . . . . . . . 135

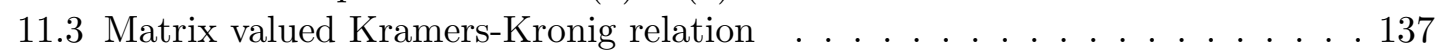

11.4 Solving the fluctuation equations $\ldots \ldots \ldots$. . . . . . . . . . . 139

IV Anomalous transport Phenomena and superfluids $\quad 141$

12 Chiral transport in Holographic Superfluids 142

12.1 Broken Anomalous symmetry . . . . . . . . . . . . . . . . . . 146

12.1.1 The Chiral conductivities in the broken phase: Axial conductivity and CEC $\ldots \ldots \ldots \ldots \ldots \ldots \ldots \ldots$

12.1 .2 The Chiral Charge Generation Effect. . . . . . . . . . . . . . . . 150

12.2 Model with axial and vector currents . . . . . . . . . . . . . . . . 151

$12.2 .1 U(1) \times U(1)$ model with transverse supervelocity . . . . . . . . 155

12.3 Conclusions, Educated guesses and Future directions . . . . . . . . . . . . 155

12.3.1 On the Low temperature behaviour of the Chiral Conductivities . . 157

12.4 Computing the Conductivities . . . . . . . . . . . . . . . . 161

12.4.1 $U(1)$ model: $\mathcal{A}_{I J}$ and $\mathcal{B}_{I J}$ matrices . . . . . . . . . . . . 161

$12.4 .2 U(1) \times U(1)$ model: $\mathcal{A}_{I J}$ and $\mathcal{B}_{I J}$ matrices $\ldots \ldots$. . . . . . . 162

12.5 Equations of Motion . . . . . . . . . . . . . . . . 163

12.5 .1 Momentum transverse to the supervelocity for the $U(1)$ model $\ldots 163$

12.5.2 Momentum parallel to the supervelocity for the $U(1)$ model . . . . . 164

12.5.3 Momentum transverse to the supervelocity for the $U(1) \times U(1)$ model 165

13 General conclusions and outlook of the work 166

$\begin{array}{lr}14 \text { Conclusiones generales del trabajo } & 170\end{array}$

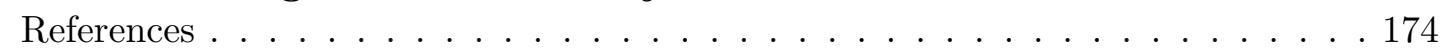




\section{List of Figures}

2.1 The regimes of validity of the two main approaches to out of equilibrium phenomena, in terms of the amplitude (A) and momentum (k) of the external perturbations. The figure has been taken from [1]] . . . . . . . . 23

3.1 The 1-loop diagram that contributes at leading order to the CVC. . . . . . 34

4.1 The spectrum of the field theoretical model. Below the critical value $\mu=M$ there are four massive modes. The masses are $M-\mu$ and $M+\mu$, the numbers indicate that they are doubly degenerate. In the broken phase $\mu>M$ there are two Goldstone modes with exactly zero mass and two gapped modes. The special gapped mode has mass $2 \mu$. . . . . . . . . . . . . . . 43

8.1 (Left) The free energy of the trivial (blue) and condensate (red) background solutions at low temperatures, $T<T_{c}$. (Right) Value of the condensate in the grand canonical ensemble as a function of $T / T_{c} . \ldots \ldots 6$

8.2 Real (left) and imaginary (right) parts of the lowest scalar QNMs as a function of the chemical potential. Solid lines correspond to the unbroken phase. For the broken phase dashed lines stand for modes of the additional scalar while dotdashed lines represent the modes common to the $U(1)$ holographic

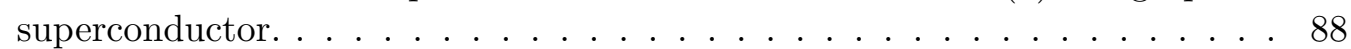

8.3 Speed of sound and damping for the sound mode. The speed of sound goes to zero at the critical temperature. The damping constant first rises quickly and then falls off again. Precisely at the critical temperature its value is such that the sound modes connect continuously to the scalar modes that become massless there. The peak in the damping constant sits close to the

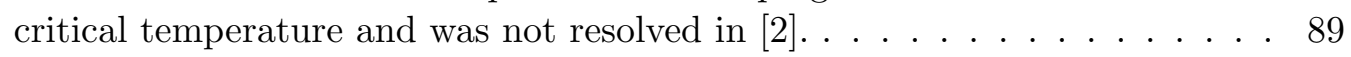

8.4 Dispersion relations of $\operatorname{Re} \omega$ (left) and $\operatorname{Im} \omega$ (right) at $T=T_{c}$ for the type I Goldstone boson in the system studied by 2 . The behavior $\operatorname{Re} \omega \sim k$ becomes quadratic right at this temperature: $\operatorname{Re} \omega=\bar{b} k^{2}$. The coefficient is $\bar{b}=0.22$, which in turn matches the value that one finds if approaches $T_{c}$ from above (i.e. from the unbroken phase). . . . . . . . . . . . . 90

8.5 (Left) Dispersion relation of the gapped pseudo diffusion mode in the broken phase for three different temperatures. The gap widens as the temperature is lowered. (Right) Gap $\gamma$ as a function of the reduced temperature $T / T_{c}$. As one approaches the critical temperature from below the gap vanishes

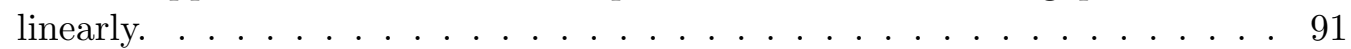


8.6 (Left) Continuation of the second and third scalar QNM into the broken phase. The real part grows as the temperature is lowered whereas the imaginary part shows very little dependence on $T$. (Right) The gap $\gamma$ (blue line) and the imaginary part of the lowest (scalar) mode fluctuation (red line) in the broken phase are shown as function of $T / T_{c}$. At $T_{*} \approx 0.69 T_{c}$ the imaginary parts cross. For lower temperatures the late time response is not dominated anymore by the pseudo diffusion mode and consequently is in form of a exponentially decaying oscillation. . . . . . . . . . . . . . 992

8.7 Real (left) and imaginary (right) parts of the type II Goldstone mode as a function of the momentum for $T / T_{c}=0.9998$ (blue) and $T / T_{c}=0.704$ (red) . The solid lines correspond to the numerical result while the dashed lines are the quadratic fit to a dispersion relation $\omega_{I I}=b k^{2}-i c k^{2} . \quad \ldots .92$

8.8 Coefficients of the type II Goldstone mode dispersion relation $\omega_{I I}=b k^{2}-$ $i c k^{2}$, as a function of the temperature. Dependence with temperature is

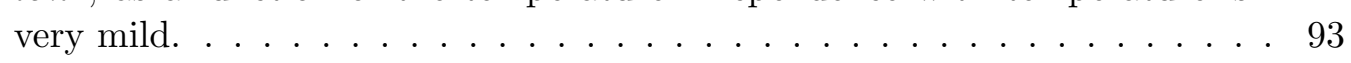

8.9 Charge density of $\Theta, \bar{n}_{\Theta}$, as a function of the temperature $T / T_{c}$. . . . . . 96

8.10 Real part (left) and imaginary part (right) of the conductivity as a function

\begin{tabular}{|c|}
\hline of frequency. The plots correspond to temperatures in the range $T / T_{c} \approx$ \\
\hline $0.91-0.41$, from red to purple. As expected, the plots reproduce the ones \\
\hline
\end{tabular}

\begin{tabular}{|c|}
\hline of frequency. The plots correspond to temperatures in the range $T / T_{c} \approx$ \\
\hline $0.91-0.41$, from red to purple. As expected, the plots reproduce the ones \\
\hline
\end{tabular}

\begin{tabular}{|c|c|c|c|}
\hline of frequency. The plots correspond to temperatures in the range $T / T_{c} \approx$ \\
\hline $0.91-0.41$, from red to purple. As expected, the plots reproduce the ones \\
\hline of $3 . \ldots \ldots \ldots \ldots \ldots \ldots \ldots \ldots \ldots \ldots \ldots \ldots \ldots \ldots \ldots \ldots \ldots \ldots \ldots \ldots \ldots \ldots \ldots \ldots \ldots$
\end{tabular}

\section{(12}

\begin{tabular}{|c|c|}
\hline & a function of the momentum for $T / T_{c}=0.9998$ (blue) and $T / T_{c}=0.704$ \\
\hline & (red) . The solid lines correspond to the numerical result while the dashed \\
\hline & lines are the quadratic fit to a dispersion relation $\omega_{I I}=b k^{2}-i c k^{2}$. \\
\hline 8.8 & Coefficients of the type II Goldstone mode dispersion relation $\omega_{I I}=b k^{2}-$ \\
\hline & $i c k^{2}$, as a function of the temperature. Dependence with temperature is \\
\hline & very mild. \\
\hline 8.9 & Charge density of $\Theta, \bar{n}_{\Theta}$, as a function of the temperature $T / T_{c}$. \\
\hline
\end{tabular}

8.11 Real (left) and imaginary (right) parts of $\sigma^{11}$ versus $\omega$ for five different

\begin{tabular}{|c|c|c|}
\hline temperatures chosen in a range $T / T_{c} \approx 0.91-0.41$, from red to purple. \\
\hline $\operatorname{Im}\left(\sigma^{11}\right)$ clearly blows up as $\omega \rightarrow 0 . \ldots \ldots \ldots 1$
\end{tabular}

8.12 Residue at $\omega=0$ as a function of $T / T_{c} . \ldots \ldots \ldots 10 \ldots \ldots 1$

8.13 Real (left) and imaginary (right) part of $\sigma^{12}$ as a function of $\omega$ for $T / T_{c} \approx$

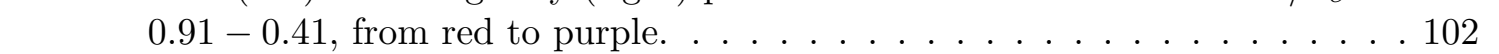

8.14 Real (left) and imaginary (right) part of the conductivity $\sigma_{-+}$for temperatures in the range $T / T_{c} \approx 0.91-0.41$, from red to purple. $\ldots . . . . .103$

8.15 Real (left) and imaginary (right) part of the conductivity $\sigma_{+-}$for temperatures in the range $T / T_{c} \approx 0.91-0.41$, from red to purple. $\ldots \ldots \ldots 104$

8.16 Plots of $\operatorname{Re}(\omega)$ (left) and $\operatorname{Im}(\omega)$ (right) as a function of the momentum. Thick lines correspond to data and thin lines to quadratic fit. At $T=$ $0.995 T_{c}$ the real quadratic parameter $\mathcal{B}(T)$ shows a maximum, see Figure 8.17. Relation (8.2.92) is fulfilled with high accuracy. . . . . . . . . . . 105

$8.17 \mathcal{B}$ (left) and $\mathcal{C}$ (right) as a function of $T / T_{c}$. The zoom-in shows the peak of $\mathcal{C}$ close to the transition. Furthermore at $T \simeq 0.4 T_{c}$ a sharp peak shows up in both coefficients. We relate this feature also to the instability arising in the vector sector. . . . . . . . . . . . . . . . 105

$8.18 \operatorname{Im} \omega$ versus $\operatorname{Re} \omega$ at $k=0$ as a function of the temperature. The shape of the figure is compatible with $T$ symmetry, since there are two pseudo-diffusive modes. Having $\operatorname{Re} \omega(k=0) \neq 0$ is characteristic of the non-Abelian case. . . 107

8.19 Real (left) and imaginary (right) part of $\mathcal{M}(T)$ as a function of $T / T_{c}$. As the temperature approaches $T_{c}$, the value of $\mathcal{M}(T)$ reaches the one prescribed

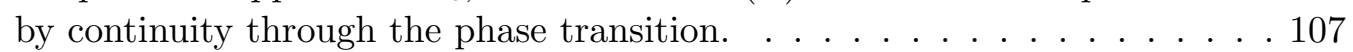

8.20 Real (left) and imaginary (right) part of the special gapped mode versus the chemical potential. We encounter the expected linear behavior with $\mu$. The plot covers both the unbroken (dashed line) and the broken (solid line)

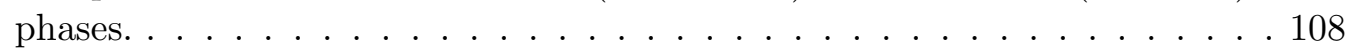


9.1 The condensate for different values of the superfluid velocity, ranging from $\frac{S_{x}}{\mu}=0.005$ (right) to $\frac{S_{x}}{\mu}=0.530$ (left). $\ldots \ldots \ldots \ldots \ldots \ldots 114$

$9.2 \quad$ Free energy of the condensed (solid line) and normal (dashed line) phases for $\frac{S_{x}}{\mu}=0.5$ (left) and $\frac{S_{x}}{\mu}=0.05$ (right). The small plots show the behavior of the condensate. The open circle corresponds to the critical temperature $T$ whereas the filled circle corresponds to the spinodal point (max. overheating). 114

9.3 Sound velocity and damping for $T=0.7 T_{c}$ and several superfluid velocities from $S_{x} / \mu=0$ (blue) to $S_{x} / \mu=0.325$ (green). The radius represents the absolute value of the sound velocity (left) and attenuation constant (right) as a function of the angle $\gamma$ between the momentum and the superfluid

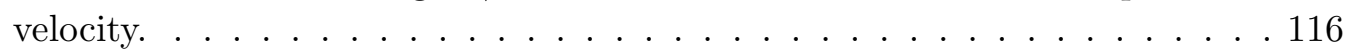

9.4 Sound velocity (left) and attenuation constant (right) for $S_{x} / \mu=0.2$ as a function of the angle $\gamma$ and for a range of temperatures from $T=0.85 T_{c}$

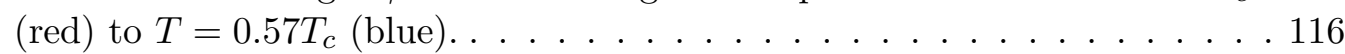

9.5 Real (left) and imaginary (right) parts of the frequency of the lowest hydrodynamic mode (type I Goldstone mode) versus momentum at $S_{x} / \mu=0.1$ and $\gamma=\pi$ for different temperatures from $T=\bar{T}=0.970 T_{c}$ (red) to $T=0.905 T c$ (blue). The instability appears at $T^{*}=0.935 T_{c} . \ldots \ldots 117$

9.6 (Left) Phase diagram after the study of the QNMs . The grey dashed line corresponds to $\tilde{T}$, the apparent transition temperature found by direct analysis of the free energy. At a certain point (disk) the transition in free energy changes from 2nd order (dotted) to 1st order (dash-dotted). The black solid line corresponds to the critical temperature in absence of superfluid velocity. The black dashed line signals the physical phase transition at $T^{*}$, the temperature at which the local instability appears. Points 1, 2 and 3 indicate the values of temperature and velocity used in the plot on the right. (Right) Imaginary part of the lowest QNM for different temperatures at fixed $S_{x} / \mu=0.2$ and $\gamma=\pi$. Dashed lines were obtained in the normal phase whereas solid lines were calculated in the superfluid phase. . . . . . . 118

9.7 Plots of the Real (left) and Imaginary (right) parts of the conductivity for fixed $S_{x} / \mu=0.05$. Different lines correspond to different temperatures from $T=0.99 T_{c}$ (red) to $T=0.38 T_{c}$ (blue). $\ldots \ldots \ldots \ldots \ldots \ldots \ldots \ldots \ldots$

9.8 Real (left) and imaginary (right) parts of the conductivity for fixed $S_{x} / \mu=$ 0.4. Different lines correspond to different temperatures in the range $T=$

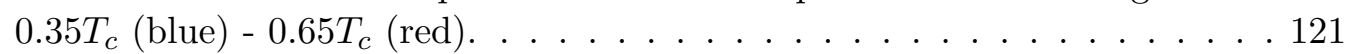

9.9 Real (left) and imaginary (right) parts of the dispersion relation of the lowest QNM of the (1)-(2) sector in the gauged model for fixed $S_{x} / \mu=0.15$ and a range of temperatures from $T=T=0.95 T_{c}$ (red) to $T=0.45 T_{c}$ (blue) and momentum anti-parallel to the superfluid velocity. . . . . . . . . 121

9.10 Real (left) and imaginary (right) parts of the dispersion relation of the lowest QNM in the (1) - (2) sector of the ungauged model for fixed $S_{x} / \mu=$ 0.25 and a range of temperatures from $T=T=0.853 T_{c}$ (red) to $T=$ $0.306 T_{c}$ (blue). Momentum is taken anti-parallel to the superfluid velocity. .122

10.1 Condensates $\psi^{(2)}$ (solid) and $w^{(1)}$ (dashed) as a function of $1 / \mu$ in the $s$ wave (blue) and $s+p$-wave (red) phases. The $p$ condensate appears at $\mu_{s p}$ such that $\mu_{s} / \mu_{s p}=0.395$ as found in Section 8.2 .5 . The inset zooms in on the plot of $\psi^{(2)}$ to show the difference in the scalar condensate between the $s$ (blue) and the $s+p$ (red) solutions. . . . . . . . . . . . . 126 
10.2 Free energy of the different solutions versus $1 / \mu$ : normal phase in black, $s$-wave phase in blue, and $s+p$-wave phase in red. . . . . . . . . . . 127

10.3 Phase diagram of the unbalanced system as a function of $1 / \mu$ and $\mu_{3} / \mu$. Second order phase transitions are denoted by blue lines, whereas the red line corresponds to a first order phase transition. . . . . . . . . . . . . 128

10.4 Free energy as a function of $1 / \mu$ for $\mu_{3} / \mu=-1$. Black corresponds to the normal phase, blue to the $s$-wave phase, and green to the $p$-wave phase. . . 129

12.1 Axial conductivity divided by the chemical potential and the anomaly coefficient versus chemical potential. $\mu_{5 c}^{*}$ is the critical chemical potential at zero supervelocity and $m^{2}=-7 / 2$. (Left) Each line corresponds to a different superfluid velocity, from $\xi_{x} / T=0.1$ (blue) to $\xi_{x} / T=2.1$ (orange). The dashed horizontal line corresponds to the unbroken phase, where $\sigma_{55} \sim \mu_{5}$. In the broken phase this conductivity approaches $1 / 3$ of the unbroken phase value for large enough chemical potential. This is compatible with the results of 44 . (Right) Each line corresponds to a different value for the mass (red $m^{2}=-7 / 2$, blue $m^{2}=-3$, green $m^{2}=-5 / 2$ ) of the scalar field in the bulk. As one can see the $1 / 3$ factor is unaltered by the dimension of the operator that condenses. The conductivity depends linearly with $\kappa . \quad$. . 147

12.2 (Left) Chiral electric conductivity versus chemical potential. Each line corresponds to a different superfluid velocity, $\xi_{x} / T=0.1-2.1$. We observe that $\sigma_{C E E} / T=0$ at $\mu_{5 c}$ and it approaches a constant value at low temperatures/ large chemical potential. (Right) Dots correspond to $\sigma_{C E E} / \kappa T$ versus $\bar{\xi}_{x}$ in the region in which $\sigma_{C E E} / T$ is independent of $\mu_{5}$. The solid line corresponds to a linear fit; the slope is 2.667. The conductivity depends

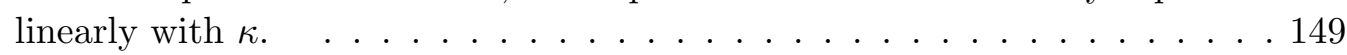

12.3 (Left) Chiral charge generation conductivity versus chemical potential, different lines correspond to different values of the supervelocity, $\xi_{x} / T=$ $0.1-2.1$. (Right) Dots correspond to $\sigma_{C^{2} G E}$ versus supervelocity for large values of the chemical potential. The solid line corresponds to a linear fit; the slope is 2.667. The conductivity depends linearly with $\kappa$. . . . . . . . 150

12.4 (Left) Axial conductivity versus vector chemical potential at $\bar{\mu}_{5}=1$ and $\xi_{x} / T=0.1-2.1$. We find that $\sigma_{55}$ is independent of both the vector chemical potential and the superfluid velocity. (Right) $\sigma_{55}$ versus axial chemical potential. The dependence is linear, as expected. The conductivity depends linearly with $\kappa . \ldots \ldots \ldots \ldots$. . . . . . . . . . . . . . . . . . . . .

12.5 (Left) Chiral separation conductivity divided by vector chemical potential versus vector chemical potential, $\bar{\mu}_{5}=1$ and $\xi_{x} / T=0.1-2.1$. The conductivity now approaches $1 / 2$ of the value at $\bar{\mu}_{c}$, independently of $\xi_{x} / T$. (Right) The plot shows this conductivity against the axial chemical potential for generic values of $\mu . \sigma_{C S E}$ is independent of the axial chemical potential in both the broken and ubroken phases. The conductivity depends

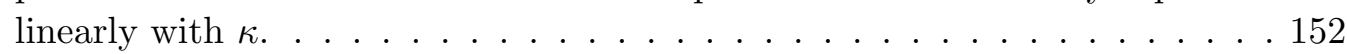

12.6 (Left) Chiral magnetic conductivity versus vector chemical potential with $\bar{\mu}_{5}=1$. Different lines correspond to different values of the superfluid velocity, with $\xi_{x} / T=0.1-2.1$. The best fit shows that for large enough values of $\bar{\mu}$ it decreases as $\sigma \sim 1 / \bar{\mu}^{2}$. (Right) This plot corresponds to $\sigma_{C M E} / T$ vs. axial chemical potential with $\mu / T=2.5$. The linear dependence with $\mu_{5}$, characteristic of the unbroken phase, remains unaltered. The conductivity depends linearly with $\kappa . \ldots \ldots \ldots \ldots \ldots$. . . . . . . . . . . . . . . . 
12.7 (Left) Chiral electric conductivities versus vector chemical potential at $\bar{\mu}_{5}=$ 1 and $\xi_{x} / T=0.1-2.1$ (bottom to top). Both $\sigma_{C E E}^{V} / T$ and $\sigma_{C E E}^{A} / T$ show the same behaviour. (Right) Chiral electric conductivities versus supervelocity at $\bar{\mu}_{5}=1$ in the region where they don't depend on $\mu$. The conductivity depends linearly with $\kappa . . \ldots$. . . . . . . . . . 154

$12.8 \sigma_{C^{2} G E}^{(A)} / T$ (Left) and $\sigma_{C^{2} G E}^{(V)} / T$ (Right) versus vector chemical potential at $\bar{\mu}_{5}=1$ and $\xi_{x} / T=0.1-2.1$ (bottom to top). For large enough values of the chemical potential both conductivities show the same behaviour. Both

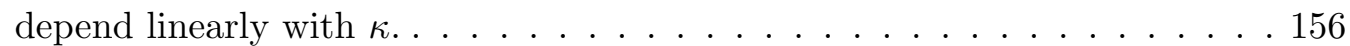

12.9 Both conductivities $\sigma_{C^{2} G E}^{(A, V)}$ show the same dependence on the vector chemical potential $\mu$ and the supervelocity $\xi_{z}$ for large enough values of $\mu$. The slope coincides with the slope for the CEC, despite the radically different behaviour close to the phase transition. . . . . . . . . . . 157

12.10Plot of the $\sigma_{C^{2} G E(V V)}$ conductivity (defined in the text) versus vector chemical potential for several values of the supervelocity. . . . . . . . 158

$12.11 \sigma_{C E E}^{(A)} / T$ (Left) and $\sigma_{C E E}^{(V)} / T$ (Right) versus vector chemical potential at $\bar{\mu}_{5}=1$ and $\xi_{x} / T=0.1-2.1$ (bottom to top) computed in the two different kinematic limits allowed by gauge invariance. For large enough values of the chemical potential the lines overlap. Notice that one of the limits corresponds to the CCGC. . . . . . . . . . . . . . . 160 


\section{Preface}

This thesis is about the application of the AdS/CFT correspondence to Condensed Matter Physics (CMP). Concretely, it focuses mainly on the study of certain exotic strongly coupled superfluids at strong coupling and their out-of-equilibrium properties, as well as the understanding of the implications of the so-called anomalous conductivities from the holographic point of view.

The AdS/CFT correspondence establishes an equivalence between string theory in a AdS background and a quantum field theory living on the boundary of AdS, with the peculiarity of being a strong/weak coupling duality. This appealing feature provides us with a powerful tool to understand strongly coupled field theories by means of perturbative calculations in string theory or its classical low energy description, supergravity. The description of the physics at strong coupling is one of the most important and unresolved problems in High-Energy Physics. At finite temperature and/or density, the understanding of the strong coupling regime has interesting applications to confinement, Quark-Gluon Plasma (QGP), High- $T_{c}$ superconductors or non-Fermi liquids, among others. This is the reason why the AdS/CFT correspondence has attracted so much attention in the last decade.

The first and more direct application of the AdS/CFT correspondence one could think of concerns the study of the QGP. But several years ago it was also realized that an even better motivated focus of the correspondence is the analysis of systems in the framework of Condensed Matter Physics. There exist a broad number of strongly correlated condensed matter systems that cannot be treated by means of usual techniques valid for weakly coupled configurations. Examples of this include the dynamics close to a Quantum Critical Point, the physics of non-Fermi liquids or the study of High- $T_{c}$ superfluids and superconductors. On the other hand, contrary to the case of the QGP, in CMP one does not usually works with a one fundamental theory but with a large variety of effective lagrangians. This opens the possibility of using experimental techniques in order to engineer a condensed matter system whose effective theory has a gravity dual. To conclude, the study of AdS/CMP correspondence appears as a very exciting and rich topic. In this thesis we will apply the correspondence to model simplified condensed matter systems and extract certain results valid in the strong coupling regime of the dual QFT.

One of the most striking and recent discoveries, for which the AdS/CFT correspondence has played a mayor role, is that of the existence of transport properties intrinsically associated to the anomalies present in the dual quantum field theory. In particular, it is now known that external magnetic field/vorticity generate a current parallel to them; the coefficients of proportionality are called anomalous or chiral transport conductivities and present very robust properties, like being non-dissipative (they are actually equilibrium quantities) and being completely fixed by the anomaly coefficient of the theory (they are universal). Chiral conductivities have been recently shown to have important consequences in the response of QGP, Weyl semimetals or superfluids. In the latter case it is known that anomalous conductivities are not anymore universal due to the presence of 
the condensate. Moreover, there arise new types of anomalous transport which induce a current in the presence of an external electric field or a finite charge density when the supervelocity is aligned with the magnetic field. Remarkably, at zero temperature these superfluid chiral conductivities are expected to be universal again. In this thesis we will present several holographic models that account for this new type of transport, both for superfluids and non-superfluids.

On the other hand, one of the main recent applications of holography to CMP is the construction of phases of matter at strong coupling. Transport properties and the spectrum of quasiparticle excitations are crucial to understand the response of such phases to small external perturbations. Probably the most famous example of this is the strongly coupled superfluid, which can be thought of as a toy model for a High- $T_{c}$ superfluid. In this thesis we present the dual of the strongly coupled two-component superfluid, which is known to feature certain exotic Goldstone modes known as Type-II Goldstone bosons (TIIGB). The existence of a TIIGB motivated us to study the Landau criterion at strong coupling in our holographic setup. The Landau criterion is a classic result in superfluids which establishes that there is a critical superfluid velocity above which the superfluid is not stable anymore. Moreover, we also construct a $\mathrm{p}+\mathrm{s}$-wave holographic superfluid, in which both a vector and scalar condensates coexist.

This thesis is divided in four parts. The first of them is an introduction devoted to present the background ingredients and techniques that will be used later on throughout the text. These include

Chapter 1 We elaborate on the gauge/gravity duality. We comment on the holographic dictionary, the possibility of calculating correlators and analyze how global currents and corresponding chemical potentials in the boundary theory are introduced. We also mention several subtleties related to the computation of thermal Lorentz correlators and briefly discuss the bottom-up methodology. We end up devoting some words to the holographic superconductor. The point of view is very practical, trying to cover the material that will be of use for the rest of the work.

Chapter 2 We discuss the well-established techniques that account for the response of a thermalized, finite density system to external perturbations. When considered in full generality, this is an unfeasible problem and hence some degree of approximation is needed. The first one is Hydrodynamics, in which we assume that the external perturbations vary very little in space and time. The second one is Linear Response Theory, which deals with perturbations that have very small amplitude. In this chapter we present both approximations, putting especial emphasis on the latter, and analyze how they can be implemented in holography. We finish the chapter focusing on an overlapping region in which booth Hydrodynamics and Linear Response Theory are good approximations to describe out-of-equilibrium physics.

Chapter 3 We analyze anomalous or chiral transport phenomena, mostly at weak coupling. After presenting the main effects, termed Chiral Magnetic and Chiral Vortical Effects, we move to the presentation of the concept of anomaly, which we do very briefly. We show later on the subtleties associated to the implementation of a chemical potential for an anomalous symmetry, due to the fact that this it is not conserved anymore. Once all this is done, we find ourselves in position of discussing anomalous Hydrodynamics (the combination of usual Hydrodynamics and anomalous transport phenomena) and the Kubo 
formulae associated to chiral conductivities, which is to say, how to use Linear Response Theory to describe anomalous transport.

Chapter 4 Here we present the concept of a superfluid. We describe how it is related to the presence of a condensate and the limitations to superfluidity imposed by the wellknown Landau Criterion. The ideal Hydrodynamics of a superfluid at zero temperature is also commented on, as well as the Meissner effect, which states that a magnetic field passing through a superconducting sample is exponentially suppressed. The spectrum of excitations is also analyzed and several generalizations are remarked. Then we use a toy model at zero temperature to derive the existence of a type II Goldstone boson. These exotic modes will be on of our subjects of study at strong coupling, using holography. Finally, we mention few properties of superfluid anomalous hydrodynamics for it will be useful for future applications.

The second part of this thesis is focused on anomalous transport at strong coupling, using the AdS/CFT. In essence, it contains the strong coupling generalization of the topics discussed in Chapter 3. From here on, the thesis correspond to original work.

Chapter 5 We use a holographic bottom-up model to compute all the anomalous conductivities in a system at finite temperature and finite density. In particular, we include the mixed gravitational anomaly and show that it accounts for the temperature dependence of the Chiral Vortical Conductivity. We also observe that strong and weak coupling results for the conductivities coincide. This implies that its value does not get renormalized as long as the gauge fields coupled to the anomaly are non dynamical.

Chapter 6 We analyze the cutoff flow of chiral conductivities by means of the botton-up model used in Chapter 5. We present several equivalent methods to compute such a flow. Due to complications induced by the mixed anomaly we stick to the most powerful method when computing the flow induced by it. On top of that, it is necessary to introduce some ad-hoc techniques in order to be able to compute two-point functions at finite cutoff in the presence of the mixed anomaly because Diritchlet B.C. become problematic.

Chapter 7 It is devoted to pointing out conclusions that can be extracted from the results of Chapters 5 and 6 .

Part 3 of this thesis studies exotic holographic superfluids. In particular, we construct the dual of a two-component superfluid and analyze its properties. We also present the construction of a $\mathrm{s}+\mathrm{p}$ - holographic superfluid, in which a scalar and vector condensates coexist.

Chapter 8 This extensive chapter serves to introduce a holographic superconductor in which a $U(2)$ symmetry is broken spontaneously to a remaining $U(1)$ symmetry. The consequence of that more complicated pattern of non-abelian broken symmetries is that the spectrum of excitations contains a type II Goldstone boson, which is a massless mode with a typically non-relativistic dispersion relation. Surprisingly enough, such a mode can also be encountered for a model in which the $U(2)$ symmetry does not have a operator realization in the boundary (what we have termed the ungauged model). Along the way we examine many properties of this superfluid, such as conductivities and diffusive modes, 
emphasizing the differences that arise when comparing them with the corresponding quantities of the usual holographic superconductor. There is also a gapped mode in this system whose mass has been shown to be protected against quantum corrections. We explicitly perform a computation of that gapped mode in our holographic model.

Chapter 9 Type II goldstone boson should prevent the system to accommodate supervelocity and hence to superfluid, according to the Landau criterion. The reason has to do with its particular non-relativistic dispersion relation. This fact motivated us to study the Landau Criterion at strong coupling to address the question of whether it holds and, if yes, what is the mechanism that gives rise to it. To that end we considered the model of Chapter 8 and study it in the presence of finite supervelocity. We observe that massless excitations are the main characters because they are responsible for triggering the Landau Criterion in holography.

Chapter 10 One of the main surprises we found when analyzing the model of Chapter 8 is that the two-component superfluid becomes unstable at some temperature. The form of the instability suggests that the new phase will contain a vector condensate. Chapter 10 is devoted to the construction of a phase with a scalar (s-wave) and vector (p-wave) coexisting condensates, that we call $\mathrm{s}+\mathrm{p}$-superconductor. We show that this is the thermodynamically preferred phase for the model of Chapter 8 at low temperatures.

Chapter 11 Here we summarize the results that have arisen in Part 2.

The last piece of this thesis, contained in Part 4, mixes somehow the work presented in Parts 2 and 3. We compute the anomalous transport in a holographic $U(1)$ superfluid, making use of Kubo formulae.

Chapter 12 Firstly we present our main expectations and certain technical subtleties. Then we construct the 5-dimensional s-wave holographic chiral superconductor and compute the anomalous conductivities. Apart from Chiral Magnetic and Vortical effects present in ordinary fluids, superfluids support two new types of chiral transport, namely Chiral Electric and Chiral Charge Generation Effects. The existence of the latter effect had not been emphasized before and we point out its importance in this chapter. Contrary to the case of ordinary fluids, we observe that anomalous transport coefficients are not universal in superfluids. However, all anomalous conductivities seem to recover universality at zero temperature. We point out that this universal value only depends on the interplay between anomalous and broken symmetries. We finish providing some considerations concerning the zero temperature picture suggested by our results.

The first part of this work is essentially a compendium of many papers and reviews. The original work presented here corresponds to Parts 2, 3 and 4. 


\section{Part I}

\section{Introduction}




\section{Chapter 1}

\section{Remarks on gauge/gravity duality and the Holographic Dictionary}

The gauge/gravity duality, holography, or more specifically the AdS/CFT correspondence, is one of the most remarkable and surprising developments in the last five teen years in theoretical physics. Its formulation by Maldacena [5] and subsequent fomalization 6,7] resulted in the culmination of previous advances in the relation between quantum gravity and non-gravitational quantum field theories (QFT) (the so-called Holographic principle 8,9 ) and the connection of large $N$ field theories with string theory [10. In retrospective, one can also identify several studies that anticipated the existence of the AdS/CFT correspondence, such as the fact that Black-Holes have entropy and temperature [11] or the identification of the asymptotic symmetries of $A d S_{3}$ and the computation of entropy from asymptotic data 12,13.

For practical purposes, the weak version of the correspondence is the most interesting one, because it is the one which is better understood. It states that a classical supergravity theory on asymptotically Anti-de-Sitter (AdS) space is dual to a gauge theory with a UV fixed point living on the (conformal) boundary of AdS (i.e. in onw dimension less), under the assumption of certain limits affecting the parameters of the theory. The canonical formulation of Maldacena concerned the duality between Type IIB supergravity in $A d S_{5} \times S_{5}$ and $\mathcal{N}=4$ Super Yang Mills, which is a Conformal Field Theory (CFT) even quantum mechanically, in the limit of large 't Hooft coupling $\lambda$ and large $N$. The gauge/gravity duality can be extended to theories lacking a UV fixed point, but we will not be concerned with that possibilities throughout this work. For very extensive reviews of the AdS/CFT correspondence, we refer the reader to $[14-19]$ and references therein.

What makes the duality extremely useful and powerful (and also very difficult to prove) is that it is a weak/strong duality, meaning that it relates a weak coupling regime in supergravity to a strongly coupled CFT and vice versa. This feature makes it specially adequate to study strongly coupled field theories. Here we provide with an introduction of the correspondence with very practical viewpoint and putting emphasis on applications to out-of-equilibrium systems.

\subsection{The dictionary}

The main ingredients of a CFT are expectation values of possibly composite gaugeinvariant operators. On the contrary, supergravity is formulated in terms of fields. The spectrum of operators of a CFT also changes from one CFT to another, which from the supergravity side is reflected in the freedom of selecting a compact manifold on which one 
compactifies ten dimensional supergravity. It is thus important to have a prescription to relate the quantities that are natural in each side of the duality. Here we will consider a small set of fields that arise very frequently in these constructions, namely the metric $g_{\mu \nu}(x)$, a gauge field $A_{\mu}(x)$ (here we will take it to be associated to a $\mathrm{U}(1)$ symmetry for simplicity) and also a scalar field $\varphi(x)$. According to the AdS/CFT dictionary, those fields are mapped to operators of the boundary CFT: the energy-momentum tensor $T^{\mu \nu}$, a current $J^{\mu}$ and a scalar operator $\mathcal{O}$ respectively.

In the classical supergravity approximation we will stick to henceforth, the so-called Witten's prescription serves to connect the dynamics of both sides of the duality. It states that the generating functional of the CFT is equal to the classical on-shell action

$$
W\left[\Phi_{0}\right]=-S_{\text {on-shell }}^{\text {sugra }}\left[\Phi_{0}\right],
$$

where $\Phi_{0}$, interpreted as a source on the left hand side, turns into a boundary condition of the corresponding supergravity field $\Phi$ on the r.h.s. In general, one finds that $S_{\text {on-shell }}^{\text {sugra }}\left[\Phi_{0}\right]$ is has a infinite value, so strictly speaking it is necessary to consider the renormalized version of the on-shell supergravity action. The procedure by which one obtains a finite on-shell action is called Holographic Renormalization 20 22]; we will not enter into the details of the associated techniques here (we discuss briefly Hamiltonian Holographic Renormalization in Appendix 7.1).

It turns out that for asymptotically AdS spaces it is possible to perform the so-called Fefferman-Graham expansion. A generic supergravity field then reads 11

$$
\Phi(z, x)=z^{\Delta_{-}}\left(\Phi_{0}(x)+z \Phi_{1}(x)+\ldots\right)+z^{\Delta_{+}}\left(\tilde{\Phi}_{0}(x)+z \tilde{\Phi}_{1}(x)+\ldots\right) .
$$

Generically, the expansion contains infinite terms (except for $A d S_{3}$, for which the number of terms is finite). The quantities $\Delta_{+}>\Delta_{-}$are the characteristic exponents of the equation. In literature, $\Phi_{0}(x)$ is called the non-normalizable mode, whereas $\tilde{\Phi}_{0}$ is termed normalizable. Throughout this work we will be imposing Dirichlet B.C. in all cases; $\Phi_{0}(x)$ is thus usually interpreted as a source for the operator dual to the field $\Phi$, and $\tilde{\Phi}_{0}$ (actually, its renormalized counterpart) corresponds to the vacuum expectation value (VEV) of such operator ${ }^{2}{ }^{3}$. Furthermore, there is a relation between the conformal weight of the boundary operator, $\Delta$, and the mass of the supergravity fields, given by $\Delta=d-\Delta_{-}(m)$, being $d$ the dimensionality of the boundary. For instance, a scalar field with bulk mass $m$ is dual to an operator $\mathcal{O}_{\Delta}$ with $\Delta=\frac{d}{2}+\sqrt{\frac{d^{2}}{4}+m^{2} L^{2}}$ ( $L$ is the AdS radius). Plugging 1.1.2 into the equations of motion for $\Phi$, it is possible to show that all modes with weight lower than $\Delta_{+}$can be expressed in terms of $\Phi_{0}$ by solving the equations asymptotically. The normalizable mode is not determined by this asymptotic (UV) analysis; $\tilde{\Phi}_{0}$ has either to be given as an extra B.C. ${ }^{4}$ or needs to be determined from a regularity condition provided

\footnotetext{
${ }^{1}$ The Fefferman-Graham expansion can include also terms of the form $\hat{\Phi} \log z$ that are related to the Weyl anomaly. We will not take them into account here for they are not important to describe the main features of the holographic dictionary.

${ }^{2}$ Strictly speaking, the 1-point function of the boundary operator is identified with the (renormalized) canonical momentum $\pi$ conjugate to $\Phi_{0}$. The difference between $\pi$ and $\tilde{\Phi}_{0}$ is in general a local functional of the non-normalizable mode 23. We will not take into account this subtlety in what follows.

${ }^{3}$ There exist situations in which both $\Phi_{0}(x)$ and $\tilde{\Phi}_{0}$ are normalizable. In those cases the role played by the fluctuations can be chosen at will. This freedom can be potentially taken into account for the model presented in Chapter 8

${ }^{4}$ For two-derivative theories, it is clear that the Dirichlet B.C. that fixes $\Phi_{0}$ is not enough to solve the problem. Provding $\tilde{\Phi}_{0}$ at the boundary accounts for the required extra condition. However, the resulting solutions obtained by this procedure are typically non-regular in the interior and therefore meaningless. It is much better to impose directly a regularity condition in the interior (IR), as mentioned in the text.
} 
in the interior (IR). Giving such a regularity condition and in addition solving the full (non-asymptotic) EOMs amounts to determining the normalizable mode as a functional of the non-normalizable one

$$
\tilde{\Phi}_{0}\left[\Phi_{0}\right]
$$

which is to say, the VEV of the dual operator in terms of the source. The problem is solved completely if one is capable of carrying this procedure out for all supergravity fields in terms of all the non-normalizable modes; all the VEVs of the dual field theory operators would be then determined in terms of the sources.

Now, given (1.1.1), it is clear that one can obtain arbitrary boundary (euclidean) correlators by taking variations of the on-shell supergravity action

$$
\left\langle\mathcal{O}\left(x_{1}\right) \mathcal{O}\left(x_{2}\right) \ldots \mathcal{O}\left(x_{n}\right)\right\rangle=\frac{\delta^{n} S_{\text {on-shell; ren. }}^{\text {sugra }}}{\delta \Phi_{0}\left(x_{1}\right) \delta \Phi_{0}\left(x_{2}\right) \ldots \delta \Phi_{0}\left(x_{n}\right)},
$$

where we have indicated explicitly that the on-shell action must have been renormalized. The above relation holds in euclidean signature. One encounters serious difficulties to compute dynamical quantities at finite temperature, such as transport coefficients, from a naive analytic continuation from euclidean path integral. This is due to the fact that at thermal equilibrium we can compactify time and represent correlators as the sum of partial contributions from the discrete Matsubara frequencies $\omega=2 \pi i n, n \geq 1 \in \mathbb{Z}$. However, when computing hydrodynamic coefficients, we will need correlators evaluated at arbitrary real values of the frequency. The transition from a discrete set of frequencies to arbitrary real values of it turns out to be the main obstacle one has to face to compute transport coefficients using lattice simulations, making the problem almost intractable to date from the lattice perspective.

Since we will be interested in problems at finite temperature and Lorentzian signature, it is important to ensure that the analytic continuation process is undertaken correctly. We postpone the discussion to Section 2.3.1 and continue commenting on the inclusion of global symmetries and chemical potential from the holographic perspective.

\subsection{Global currents and chemical potential in holography}

The first term in a low energy expansion in gravity is the Einstein-Hilbert contribution, which can be considered as a kind of universal sector, common to any (possibly higher derivative) supergravity theory. In order to accommodate a AdS background, we need to include a negative cosmological constant. On top of that, one has to consider matter fields. Instead of sticking to some consistent compactification of supergravity, we can simply add by hand the matter fields that one wants; this procedure is often referred to as a bottom-up approach, because we do not care how the theory under consideration can be derived from a stringy construction. This is similar in spirit to the phenomenological models often worked out in condensed matter physics, in which one considers a certain simplified Hamiltonian which features some of the properties one is intended to study, without focusing so much on the microscopic realization of that Hamiltonian. After all, in holography the asymptotic symmetries of AdS plus the application of a well defined holographic renomalization procedure allows us to define consistently certain finite correlators and Ward identities at the boundary of spacetime at the formal level. The correspondence comes into help only when one wants to interpret such correlators in terms of n-point functions of a quantum CFT that lives on the boundary. We adopt this bottom-up approach 
in all of our models defined in subsequent chapters, so our holographic models will read 5

$$
S=\int d^{d+1} x \sqrt{-g}(R-2 \Lambda)+S_{\text {matter }}
$$

Typically, with Condensed Matter applications in mind, we are interested in introducing a chemical potential associated to some global symmetry, that here we will take to be a $\mathrm{U}(1)$ symmetry for simplicity. Of course, a theory at finite temperature will be even more appealing. We will explain in Section 2.1 that heating the theory up amounts to inserting an asymptotically AdS black hole in spacetime. But for the moment let us elaborate on the introduction of a $\mathrm{U}(1)$ chemical potential, assuming that the theory is already at finite temperature.

If we wanted a conserved global current $\mathcal{J}^{\mu}$ at the boundary, we would be forced to include a dynamical gauge field $A_{M}$ in the action (1.2.5). This is a particular example of the general statement that establishes that gauge symmetries in the bulk are mapped to global symmetries on the boundary. Since in our boundary lagrangian the corresponding coupling is $\int d^{d} x \mathcal{A}_{\mu} \mathcal{J}^{\mu}$, we notice that the non-normalizable mode of the temporal component of the bulk gauge field is dual to the source for the charge density, that is, the chemical potentia:

$$
A_{0}(z \rightarrow 0, x) \leftrightarrow \mathcal{A}_{0}(x) \equiv \mu(x)
$$

Usually one is interested in situations in which the background is homogeneous and thus assumes that $A_{0}(z \rightarrow 0)$ is independent of the transverse coordinates. We conclude that a bulk theory with a global U(1) symmetry on the boundary has to be of the form 1.2.5 with

$$
S_{\text {matter }}=\int d^{d+1} x \sqrt{-g}\left(-\frac{1}{4} F_{M N} F^{M N}\right),
$$

being $F=d A$ the field strength. A solution to the EOMs derived from action (1.2.5), with $S_{\text {matter }}$ given by (1.2.7), is a Reissner-Nordström black brane (see Chapter 2.1 for an explanation of why this is the adequate metric solution in the situation at hand) and a background gauge field

$$
A=\mu\left(1-\left(\frac{z}{z_{H}}\right)^{(d-3) / 2}\right) d t
$$

so that $A_{0}(z \rightarrow 0)=\mu$ as anticipated and the charge density $n=\mu / z_{H}^{2} \equiv\left\langle J_{0}\right\rangle$. Sticking to the bottom-up approach, one can consider further extensions of $S_{\text {matter. A possible }}$ generalization consists in adding a complex massive scalar field $\Psi$ dual to a scalar operator. This has remarkable consequences: by tuning the mass of the scalar, it can be shown that

\footnotetext{
${ }^{5}$ Actually, in Chapters 5 and 6 we will consider a higher derivative model to implement the mixedgauge-gravitational anomaly.

${ }^{6}$ The gauge-invariant quantity that thereby acts as the source is actually $A_{0}(z \rightarrow 0, x)-A\left(z=z_{H}, x\right)$, where $z_{H}$ is the position of the horizon. Typically the gauge field is taken to vanish at the horizon and one recovers 1.2.6; the reason for assuming $A\left(z=z_{H}, x\right)=0$ is to make sure that the field will be well-behaved when analytically continuing to euclidean space. However, in reality the gauge field is not gauge invariant and therefore not observable (this is not true for superfluids/superconductors) and regularity of $A_{M}$ at the horizon, although appealing, is not necessarily a physical requirement. We point this out because it has important implications when discussing the definition of the chemical potential in the presence of anomalies.
} 
the system undergoes a phase transition, the U(1) symmetry becoming spontaneously broken as a consequence of it. For obvious reasons, this set up has been called a (s-wave) holographic superconductor (to be more precise, the system becomes a superfluid after the phase transition due to the fact that the broken symmetry is global, i.e. there is no Higgs mechanism taking place in the boundary theory). The corresponding action for matter fields is

$$
S_{\text {matter }}=\int d^{d+1} x \sqrt{-g}\left(-\frac{1}{4} F_{M N} F^{M N}-\overline{D_{\mu} \Psi} D^{\mu} \Psi+2|\Psi|^{2} / L^{2}\right) .
$$

In the introduction of Part III we elaborate on the features of a holographic superconductor. The discussion serves as an introduction to Chapters 8 and 9 , in which we present a holographic superconductor featuring a $U(2)$ symmetry that gets spontanoeusly broken to $U(1)$. In that case $S_{\text {matter }}$ is more complicated than $(1.2 .9)$ for it features a $U(2)$ gauge symmetry and the complex scalar field transforms as a doublet of $\mathrm{SU}(2)$. We analyze the interesting physical consequences of such a more complicated pattern of broken symmetries in the aforementioned chapters. 


\section{Chapter 2}

\section{Holographic approximations to out-of-equilibrium physics}

This section is devoted to presenting the most famous approximations to out-ofequilibrium physics, meaning hydrodynamics and linear response theory. We will focus mainly on the description at strong coupling, using holographic methods. For general reviews on the application of holography to Condensed Matter Physics, see 2427 We start by giving a picture of the state of Global equilibrium. Then we move to the description of hydrodynamic local equilibrium; even though the hydrodynamic approach will not be the one adopted in the following chapters, for completeness we will give a brief detour for the hydrodynamical description of weakly and strongly coupled theories. This Section concludes with a more detailed description of Linear Response Theory in QFT and Holography.

\subsection{Global Equilibrium}

In Quantum Mechanics the grand canonical ensemble is determined by a density matrix defined on Fock space. We can write it in a covariant form

$$
\rho=Z_{G}^{-1} e^{\beta_{\mu} P^{\mu}+\beta \mu N} ; \quad Z_{G}=\operatorname{tr} e^{\beta_{\mu} P^{\mu}+\beta \mu N}
$$

where $\beta^{\mu}=\beta u^{\mu}\left(u^{\mu}=(\gamma, \gamma \vec{v})\right.$ is the four-velocity $), P^{\mu}$ and $N$ being the momentum and number operators respectively. In the rest frame $u^{\mu}=(1,0,0,0)$ and (2.1.1) reduces to a quantum-mechanical version of (??). Generically, Lorentz invariance (concretely, boost invariance) and Supersymmetry will be broken by thermal states.

\subsubsection{Global Equilibrium state as a geometric object in $A d S / C F T$}

The intuition that Black holes correspond to global equilibrium states in the dual theory could be natural even for physicists not aware of the AdS/CFT correspondence. This is due to the laws of black hole mechanics, established by Bardeen, Carter and Hawking [11, that have a strong resemblance of the laws of Thermodynamics. Moreover, in asymptotically flat spacetimes there are theorems, formulated in the $60 \mathrm{~s}$, that prove the uniqueness of several solutions under the assumption of regularity of the horizon, depending on the theory under consideration and the isometries of the black-hole. These are the Schwarzchild (Sch), Reissner-Nordström (RN) and Kerr (K) Black-Holes! 1. It turns out

\footnotetext{
${ }^{1}$ There exist also a Kerr-Reissner-Nordström BH.
} 
that these BHs can be described entirely by certain quantities measured at infinity, namely the mass, the chemical potential or the angular momentum ("BHs have no hair" [28]). This fact already suggested the relation between BHs and thermodynamic states, in which the whole system is described by a set of macroscopic variables.

Moving to asymptotically AdS spaces, one can basically map the conclusions of the previous paragraph ${ }^{2}$. In holography, the $\mathrm{Sch} \mathrm{BH}$ (actually, Black Brane (BB)) is dual to a thermal state at finite temperature in the Canonical ensemble, whereas the RN BH corresponds to the Grand-Canonical state 2.1.1). The Kerr BH corresponds to a state in which we source the angular velocity of the system, and will not be considered here. In fact, it can be shown that only asymptotically AdS-BHs reach equilibrium with their radiation for temperatures higher that a critical value $T_{c}$, turning them in good candidates for being associated to global equilibrium states. In addition to this, Hawking and Page showed that BHs undergo a first order phase transition [29], from a thermodynamically radiation-preferred phase (the thermal AdS background) for $T_{c}<T<T_{1}$ to a BH preferred phase (AdS-Sch BH) at temperatures $T_{1}<T<T_{2}$. If $T>T_{2}$, all radiation collapse into BHs.

To avoid these potential issues, in the following we will always assume that we sit in the large-temperature regime of the theory, in which we basically have AdS Sch BH only. More specifically, if the temperature in $d$ dimensions reads

$$
T=\frac{d r_{+}^{2}+(d-2) R_{A d S}^{2}}{4 \pi r_{+} R_{A d S}^{2}},
$$

where $r_{+}$is the position of the horizon and $R_{A d s}$ is the radius of AdS, thermal AdS and AdS-Sch BH exchange dominance at $r_{+}=R_{A d S}$ [1]. Going to the large $r_{+}$limit we get the temperature of the theory

$$
T=\frac{d}{4 \pi} r_{+},
$$

Henceforth we will stick to large $\mathrm{BHs}$ and hence take the above definition of temperature (the formula changes if we consider a large $\mathrm{RN} \mathrm{BH}$, due to the presence of a finite chemical potential) . One could ask how to go beyond large Black Branes (i.e. global equilibrium situations in AdS/CFT). Departing from global equilibrium in the dual field theory can be seen as perturbing the $B B$ in different ways in the bulk. In the next subsections we will describe how this is achieved.

\subsection{Hydrodynamics}

Hydrodynamics is an old discipline [30, 31. It is and effective field theory that allows us to describe out-of-equilibrium systems whenever the external perturbations fulfill $k \lambda_{m f p}<<1$, where $k$ is the momentum and $\lambda_{m f p}$ is the mean free path of the constituents $\left(k \lambda_{m f p}\right.$ can be regarded as the expansion parameter). Physically, that condition means that the distance at which the external sources vary is much larger than the mean free path of the constituents. The above condition is equivalent to stating that there is an analytic expansion in $k \lambda_{m f p}$ of the correlators of our theory; hydrodynamic constitutive relations are nothing but the formal representation of those expansions.

In theories in which we have a well-defined scale $M$ (for instance, a mass gap), $\lambda_{m f p} \sim 1 / M$

\footnotetext{
${ }^{2}$ The no-hair conjecture can be violated, however. We will see an example of this later on.
} 
and thus it is always possible to apply hydrodynamics. However, in a Conformal Field Theory (CFT) there is not such a scale. Therefore, there exist long-range correlations and typically the correlators are not analytic functions of momentum. The way out consists in heating up the theory; this would automatically imply that $\lambda_{m f p} \sim 1 / T$ and then hydrodynamics can be fomulated as an effective field theory with an expansion parameter that is the momentum of the external sources over the temperature of the system. Since in a CFT one can always set $T \equiv 1$, hydrodynamics is valid iff $k<<1$.

Here we will focus on relativistic hydrodynamics, because all our forthcoming results apply to relativistic CFTs. We start out in a global equilibrium situation, in which it is possible to define thermodynamic quantities, such as temperature $T$, chemical potential $\mu$, or velocity $u^{\mu}$. The action of external sources will in general drive the system into a complicated out-of-equilibrium state. However, if the perturbations vary very little in space-time, one can argue that there will be still certain regions where the system will be approximately in equilibrium, so we can divide our sample in patches, each of which is in thermal equilibrium and thus has well-defined thermodynamic quantities. After coarsegraining, each patch is associated to a point and our thermodynamic variables now depend slightly on the position in space-time. Notice that the whole construction requires a compromise: regions which are not in equilibrium with respect to each other must be very well sperated, such that objects like temperature can still be defined locally. This is the reason why it is usually claimed that hydrodynamics describes systems that are in local equilibrium.

Hydrodynamics requires two type of constituents: equations describing the dynamics (which in the relativistic regime are just the conservation laws) and constitutive relations, that, as aforementioned, are the expansion in momentum of the one point functions. To be specific, consider a theory containing with a well-defined energy-momentum tensor $T^{\mu \nu}$ and a current associated to a conserved $U(1)$ symmetry, $J^{\mu}$; the conservation equations read

$$
\begin{aligned}
D_{\mu}\left\langle T^{\mu \nu}\right\rangle & =F_{\rho}^{\nu} J^{\rho}, \\
D_{\mu}\left\langle J^{\mu}\right\rangle & =0 .
\end{aligned}
$$

where $D_{\mu}$ is the covariant derivative and $F_{\mu \nu}$ is the $U(1)$ stress-tensor. The simplest constitutive relations are the ideal ones

$$
\begin{aligned}
\left\langle T_{(0)}^{\mu \nu}\right\rangle & =\mathcal{E} u^{\mu} u^{\nu}+\mathcal{P} P^{\mu \nu}, \\
\left\langle J_{(0)}^{\mu}\right\rangle & =\mathcal{N} u^{\mu} .
\end{aligned}
$$

Here $P^{\mu}{ }_{\nu}=\delta^{\mu}{ }_{\nu}+u^{\mu} u_{\nu}$ is the induced metric on the hypersurface orthogonal to the timelike vector $u_{\mu}$ (so that $u_{\mu} P^{\mu \nu}=0$ and $P_{\mu}^{\mu}=3$ in four dimensions). In addition, $\mathcal{E}=\epsilon$ is the energy density, $\mathcal{P}=p$ is the pressure and $\mathcal{N}=n$ the charge density. To obtain those identifications, one has to take advantage of the fact that there is always a frame in which the $T_{(0)}^{\mu \nu}$ is diagonal and $J_{(0)}^{\mu}$ is a vector with only the first entry different from zero; such a reference frame is often called the "fluid rest frame", i.e. $u^{\mu}=(1,0,0,0)$ in it. Ideal Hydrodynamics is equivalent to thermodynamics (this can be seen by going to the rest frame); given the equations of state $\epsilon(\mu, T), p(\mu, T), n(\mu, T)$, the system is completely characterized by the chemical potential $\mu$ and the temperature. Furthermore, the conservation equations are satisfied trivially.

Henceforth, for simplicity we will write the Hydrodynamic expressions, such as 2.2.6)2.2.7, without the brackets. 


\subsubsection{The frame choice in non-ideal Hydrodynamics}

Non-ideal hydrodynamics consists in terms containing powers of the four-momentum. For instance, in the presence of an external electric field, there will be a term of the form $\delta\langle\vec{J}\rangle=\sigma \vec{E}$ in the constitutive relations. Coefficients like $\sigma$ are called "transport coefficients" generically. As in generic effective field theories, they depend on the underlying microscopic theory. First order Hydrodynamics can be obtained by allowing the thermodynamic coefficients to depend on the coordinates a little bit

$$
\left(T, \mu, u^{\mu}\right) \rightarrow\left(T(x), \mu(x), u^{\mu}(x)\right)
$$

so that

$$
\lambda_{m f p} \partial_{\mu}\left(T(x), \mu(x), u^{\mu}(x)\right)<<1 .
$$

The first order constitutive relations thus read in general 32

$$
\begin{aligned}
T^{\mu \nu} & =\mathcal{E} u^{\mu} u^{\nu}+\mathcal{P} P^{\mu \nu}+\left(q^{\mu} u^{\nu}+q^{\nu} u^{\mu}\right)+t^{\mu \nu}, \\
J^{\mu} & =\mathcal{N} u^{\mu}+j^{\mu} .
\end{aligned}
$$

In the above formulae $q^{\mu}$ and $j^{\mu}$ are transverse and $t^{\mu \nu}$ is transverse and traceless. It turns out that these properties ensure that $q^{\mu}, j^{\mu}$ and $t^{\mu \nu}$ can only be built up by at least one-derivative terms in the hydrodynamic variables. This explains why the zeroth-order expansion is prescribed just by equations 2.2.6-2.2.7.

Notice that there is not a unique definition of the spacetime-dependent hydrodynamic variables. The only microscopic operators that we have in Hydrodynamics are $J^{\mu}(x)$ and $T^{\mu \nu}(x)$ and thus we can consider definitions of $\left(T(x), \mu(x), u^{\mu}(x)\right)$ that differ by first order derivative terms

$$
\left(T^{\prime}(x), \mu^{\prime}(x), u^{\prime \mu}(x)\right)=\left(T(x)+\delta T(x), \mu(x)+\delta \mu(x), u^{\mu}(x)+\delta u^{\mu}(x)\right),
$$

where $\delta T(x), \delta \mu(x), \delta u^{\mu}(x)$ are $\mathcal{O}(k)$. The choice of $\left(T(x), \mu(x), u^{\mu}(x)\right)$ is often referred in literature as a choice of fram $\AA^{3}$. Working with the primed variables is consistent because in the pure equilibrium situation $(\omega, k)=(0,0)$ we recover the equilibrium quantities $T, \mu$ and $u^{\mu}$. Moreover, both $J^{\mu}$ and $T^{\mu \nu}$ remain the same. The transformation induced by 2.2 .12 takes the following form

$$
\begin{aligned}
\delta \mathcal{E} & =\delta \mathcal{P}=\delta \mathcal{N}=0, \\
\delta q_{\mu} & =-(\mathcal{E}+\mathcal{P}) \delta u_{\mu}, \delta j_{\mu}=-\mathcal{N} \delta u_{\mu}, \\
\delta t_{\mu \nu} & =0
\end{aligned}
$$

where we have used the fact that $u_{\mu} \delta u^{\mu}=0$. Equations $(2.2 .14)-(2.2 .15)$ tell us that we can use the arbitrariness of $\delta u^{\mu}$ to go to a frame in which $j_{\mu}=0$ (Eckart frame) or $q_{\mu}=0$ (Landau frame). For the scalars, one parametrize the transformations enforced by 2.2.12) as

$$
\begin{aligned}
\mathcal{E} & =\epsilon(T, \mu)+f_{\mathcal{E}}(\partial T, \partial \mu, \partial u) \\
\mathcal{P} & =p(T, \mu)+f_{\mathcal{P}}(\partial T, \partial \mu, \partial u) \\
\mathcal{N} & =n(T, \mu)+f_{\mathcal{N}}(\partial T, \partial \mu, \partial u)
\end{aligned}
$$

\footnotetext{
${ }^{3}$ We will adopt this terminology here, even though it is a bit misleading, because the choice of frame that we refer to in the text is not a choice of an observer, i.e. a choice of reference frame.
} 
where $\epsilon, p$ and $n$ are the energy density, pressure and charge density defined in equilibrium. Relations 2.2.13 imply that $\epsilon(T, \mu)+f_{\mathcal{E}}(\partial T, \partial \mu, \partial u)=\epsilon\left(T^{\prime}, \mu^{\prime}\right)+f_{\mathcal{E}}^{\prime}\left(\partial T^{\prime}, \partial \mu^{\prime}, \partial u^{\prime}\right)$, the same applying to $\mathcal{P}$ and $\mathcal{N}$. It turns out that we can use $\delta T, \delta \mu$ to fix two of the $f_{\mathrm{s}}$ to zero. It is frequent to choose $f_{\mathcal{E}}^{\prime}=f_{\mathcal{N}}^{\prime}=0$, which implies $\mathcal{E}=\epsilon$ and $\mathcal{N}=n$ in 2.2.10)(2.2.11) 33].

One can also assume a generic frame, where all quantities are generically different from zero. Being this so, one can realize that the vector $l^{\mu}$ and function $f$, defined as

$$
\begin{aligned}
l^{\mu} & \equiv j^{\mu}-\frac{n}{\epsilon+p} q^{\mu} \\
f & \equiv f_{\mathcal{P}}-\left(\frac{\partial p}{\partial \epsilon}\right)_{n} f_{\mathcal{E}}-\left(\frac{\partial p}{\partial n}\right)_{\epsilon} f_{\mathcal{N}},
\end{aligned}
$$

are frame-invariant 33 .

We move now to discuss hydrodynamics in holography, leaving the construction of first order Hydrodynamics to Section 3.2 .

\subsubsection{Hydrodynamics and Holography}

Even though we are not going to make use of the technics developed to study Hydrodynamcs of holographic fluids, for completeness we include here a very brief introduction to the topic, together with some references where the interested reader can find extensive information.

First of all, one could rise the question What is a holographic fluid? Typically, at large enough temperatures we expect any weakly coupled system to be describable by hydrodynamics. At strong coupling this is not so obvious; however, it seems that the claim also holds for certain class of theories with holographic duals. In the language of Effective Field Theory, we can think of Hydrodynamics as the most extremely coarse-grained approximation compatible with locality. Only massless modes survive the procedure, the resulting theory being valid at very small frequency and momentum. Thus we are left with a sector of coupled massless modes whose dynamics is prescribed by a set of constitutive relations, including some coefficients (transport coefficients) which depend on the underlying microscopic theory (the degrees of freedom that we have integrated out). The interaction between the hydrodynamic degrees of freedom and the ones that we have integrated out therefore provides the notion of dissipation in Hydrodynamics 34, 35. In holography the microscopic theory is strongly coupled, but the interaction between massless modes or the one between massless modes and the micrscopic theory can be weak in principle. In a CFT the bulk viscosity vanishes due to conformal invariance, but the shear viscosity is generically different from zero and is related to the diffusion of the microscopic degrees of freedom. The believe that this diffusion is suppressed in strongly coupled theories lead us to the conclusion that the shear viscosity must be very small. And indeed that is what is found 36

$$
\eta=\frac{s}{4 \pi}
$$

where $s$ is the entropy density of the fluid. The Quark Gluon Plasma (QGP) generated at RHIC and the LHC is an example of a strongly coupled system that can be described by Hydrodynamics. Remarkably enough, its correspondng shear viscosity to entropy density 
is of the same order of magnitude than in 2.2.21.

The relation between Black holes and Hydrodynamics (ecoded supposedly in its sketched horizon) had been anticipated long time ago 37]. One can see the idea as a natural extension of the fact that BHs behave as Thermodynamic systems. With the development of the AdS/CFT correspondence, the aforementoned relation become apparent and the program was revisited with renewed interest. This ended up with the construction of the so-called Fluid/Gravity correspondence [38]. Let us summarize its main features.

The idea beind the Fluid/Gravity correspondence is very natural (for a review, see [39]). In the same way as one can construct first order Hydrodynmics by assuming that the thermidynamical parameters $\mu, T$ and $u^{\mu}$ of ideal Hydrodynamics change slowly with position and time, we can also deform the boosted- BH solution (we boost the $\mathrm{BH}$ to force the explicit appearance of $u^{\mu}$, in the same way that Ideal hydrodynamics can be seen as a "boosted version" of thermodynamics 4 , in order for the parameters $\left(\mu, M, u^{\mu}\right) \rightarrow$ $\left(\mu(x), M(x), u^{\mu}(x)\right)$ to acquire an $x$-dependence, i.e. promoting them to Goldstone fields. This means the parameters $\beta^{\mu}$ and $\gamma$, that enter the state 2.1.1) (responsible for breaking the Lorentz symmetry), are now promoted to fields; Hydrodynamics is about the dynamics of these Goldstone modes. The arbitrary deformation of thermodynamic parameters made by hand automatically implies that the new $\mathrm{BH}$ is not anymore a solution to the Einstein equations of motion. But we can enforce our deformation to correspond to a solution; this is obtained by solving the Einstein equations order by order in derivatives 5 , i.e. solving the dynamics of the Goldstone fields. The procedure generates the Hydrodynamic expansion of the dual field theory, namely, one-point functions for the energy-momentum tensor and the conserved currents (up to anomalies). Notice that the hole construction relies on the pre-existing homogeneous BH solution; in other words, the dual CFT must be at finite temperature (or at least at finite chemical potential), as expected from our previous discussion.

\subsection{Linear response theory}

Linear response theory describes out-of-equilibrium processes in which the external perturbations, that drag the system out of the global equilibrium regime, are small in amplitude, so that, in certain sense, a perturbative expansion of the dynamical equations is siutable. Note that, contrary to hydrodynamics, there is no restriction on the value of the momentum of the external perturbations.

To be concrete, let us consider deformations of the Hamiltonian $H \rightarrow H+\epsilon H_{\text {pert. }}(t)$, being $\epsilon<<1$ a small expansion parameter. The initial Hamiltonian $H$ is time independent and the condition that the system is in global equilibrium implies $[H, \rho]=0$, with $\rho$ corresponding to the thermal density matrix. Since $\langle\mathcal{O}\rangle=\operatorname{Tr}(\rho \mathcal{O})$ (the brackets actually correspond to a thermal expectation value), we conclude that all the temporal dependence of $\langle\mathcal{O}\rangle$ is encoded in $H_{\text {pert. }}(t)$. Writing $\langle\mathcal{O}\rangle=\langle\mathcal{O}\rangle_{\text {equ. }}+\epsilon \delta\langle\mathcal{O}\rangle$, to first order in $\epsilon$ we have

$$
\frac{\partial \delta\langle\mathcal{O}\rangle}{\partial t}=-i\left\langle\left[\mathcal{O}, H_{\text {pert. }}\right]\right\rangle_{\text {equ }}
$$

\footnotetext{
${ }^{4}$ In gravity, one can see that dilatations and boosts are not isometries of the static AdS-RN Black Brane. Hence, one can generate a family of solutions parametrized by $\left(T, u^{\mu}\right)[1$.

${ }^{5}$ Actually, in momentum over temperature as aforementioned.
} 
Integrating this expression we arrive at

$$
\delta\langle\mathcal{O}\rangle(\vec{x}, t)=-i \int_{t_{0}}^{t} d t^{\prime} \operatorname{Tr}\left(\rho\left[\mathcal{O}, H_{\text {pert. }}\left(t^{\prime}\right)\right]\right)
$$

In order to recast these expressions in terms of (retarded) Green's functions, we consider a QFT, whose Lagrangian is deformed by $\Delta L=-\epsilon \int d^{3} x J(x, t) \mathcal{S}(x, t)$, being $\mathcal{S}(x, t)$ a (possibly composite) operator which does not involve time-derivatives of the fundamental fields. Then $H_{\text {pert. }}=\epsilon \int d^{3} x J(x, t) S(x, t)$. Moreover, we set $t_{0} \rightarrow-\infty$ and impose causality, i.e. the response cannot precede the excitation. With this in mind, we can write

$$
\delta\langle\mathcal{O}\rangle(x)=\int d^{4} x^{\prime} G_{R}^{\mathcal{O}}\left(x-x^{\prime}\right) J\left(x^{\prime}\right),
$$

with $G_{R}^{\mathcal{O}}\left(x-x^{\prime}\right) \equiv-i \theta\left(x_{0}-x_{0}^{\prime}\right) \operatorname{Tr}\left(\rho\left[\mathcal{O}, \mathcal{S}\left(x^{\prime}\right)\right]\right)$ being the retarded correlator. The above formula establishes the change in the expected value of an operator $\mathcal{O}$ after turning on a source $J$ that couples to $\mathcal{S}$ in the Lagrangian. Typically, one is interested in case where $\mathcal{S}$ is a conserved quantity, i.e. $[H, S]=0$ for in that case we can study linear response theory due to fluctuations in parameters such as the charge density, which are conserved under time evolution.

Let us imagine that we have a QFT defined in Minkowski space, enjoying a $U(1)$ symmetry. It is very natural to consider Green's functions of current and energy-momentum operators. Throughout this work we will be mainly interested in the following correlators

$$
\begin{gathered}
G_{\mu \nu}^{J J}\left(x-x^{\prime}\right) \equiv-i \theta\left(x_{0}-x_{0}^{\prime}\right)\left\langle\left[J_{\mu}(x), J_{\nu}\left(x^{\prime}\right)\right]\right\rangle, \\
G_{\mu \nu, \alpha}^{T J}\left(x-x^{\prime}\right) \equiv-i \theta\left(x_{0}-x_{0}^{\prime}\right)\left\langle\left[T_{\mu \nu}(x), J_{\alpha}\left(x^{\prime}\right)\right]\right\rangle .
\end{gathered}
$$

where the fact that the above correlators are retarded is understood and the expected value can be a thermal one. Consider a 4-dimensional QFT at finite temperature. The background is not sourced, so we can take the momentum to be $k^{\mu}=(\omega, 0,0, \mathbf{k})$ without loss of generality. It is possible to show that in momentum space

$$
\begin{aligned}
G_{x^{1} x^{1}}^{J J}(k) & =G_{x^{2} x^{2}}^{J J}(k)=\Pi^{T}(\omega, \mathbf{k}) ; & G_{x^{0} x^{0}}^{J J}(k) & =\frac{\mathbf{k}^{2}}{\omega^{2}-\mathbf{k}^{2}} \Pi^{L}(\omega, \mathbf{k}), \\
G_{x^{0} x^{3}}^{J J}(k) & =\frac{-\omega \mathbf{k}}{\omega^{2}-\mathbf{k}^{2}} \Pi^{L}(\omega, \mathbf{k}) ; & G_{x^{3} x^{3}}^{J J}(k) & =\frac{\omega^{2}}{\omega^{2}-\mathbf{k}^{2}} \Pi^{L}(\omega, \mathbf{k}) .
\end{aligned}
$$

The low energy limit $\omega, \mathbf{k}<<T$ of the scalar functions $\Pi^{L}, \Pi^{T}$ is fully determined by Hydrodynamics [40].

As an application, we obtain the diffusive mode, which determines how charge fluctuations propagate in the system until equlibrium is reached. To that end, we make use of conservation equation 2.2.5 plus the following expectation: to leading order in derivatives, the spatial currents generated in the system are due to inhomogeneities of the charge density $\vec{J}=-D \vec{\nabla} J^{\sqrt{6}}$. This automatically implies

$$
\partial_{t} J^{0}-D \vec{\nabla}^{2} J^{0}=0
$$

\footnotetext{
${ }^{6}$ In the presence of an anomaly and a background magnetic field, this relation has to be corrected, even at first order in derivatives. This gives rise to the so-called Chiral Magnetic Wave 41.
} 
Assuming that our system is infinite, we only need to prescribe initial conditions. Performing a Fourier transformation in space and a Laplace transformation in time

$$
J^{0}(\vec{k}, z)=\int d^{3} x \int_{0}^{\infty} d t e^{-i \vec{k} \cdot \vec{x}} e^{i z t} J^{0}(\vec{x}, t),
$$

we get, rather straightforwardly

$$
J^{0}(\vec{k}, z)=\frac{i}{z+i D k^{2}} J^{0}(\vec{k}, t=0) .
$$

Hence, we notice that there exists a diffusion-type mode with dispersion relation $z=$ $-i D k^{2}$. Solutions of the form 2.3.30 are compatible with diffusion of charge through random walk-type processes [40], being $D$ the so-called diffusion constant. The diffusive mode belongs to the longitudinal sector, i.e. arises as a pole in $\Pi^{L}(\omega, \mathbf{k})\left(\Pi^{T}(\omega, \mathbf{k})\right.$ does not contain any hydrodynamic pole). Remarkably enough, this conclusion can be reached by means of holography, getting

$$
\omega(\mathbf{k})=-i D \mathbf{k}^{2} ; \quad D=1 /(2 \pi T)
$$

\subsubsection{Linear Response Theory in Holography}

The strength of the gauge/gravity correspondence is that it allows to study the real time dynamics of strongly coupled field theories rather easily. Linear response theory captures the behavior of a quantum system after an initial, small perturbation. It also applies to the late time behavior when an initially large perturbation has already sufficiently died out and enters the linear regime. The basic ingredient of linear response theory, as we have seen, is the retarded Green's function. As mentioned in Section 1.2, at finite temperature one has to be careful when defining retarded correlators 42,43. From the point of view of holography, there are also several differences that arise from passing from euclidean to lorentzian time.

Let us consider a field (for simplicity, we can picture it as an scalar field) in the background of a AdS-Sch BH. In euclidean signature, the Fourier-transformed field at the horizon behaves as

$$
\Phi_{\text {Eucl. }} \sim\left(r-r_{H}\right)^{ \pm \omega /(4 \pi T)},
$$

so that imposing regularity at the $\mathrm{BH}$ orizon $r_{H}$ implies picking the minus sign solution. Now, in lorentzian signature the possible solutions at the horizon read

$$
\Phi_{\text {Lor. }} \sim\left(r-r_{H}\right)^{ \pm i \omega /(4 \pi T)} .
$$

The two solutions feature now a constant amplitude as we approach $r_{H}$ and hence are both regular and physical. The freedom in choosing the + or - sign corresponds to the possibility of computing advanced or retarded correlators, respectively, from the boundary theory point of view. This can be seen by adding the $e^{-i \omega t}$ part of the fourier transformation

$$
\begin{aligned}
& e^{-i \omega t} \Phi_{\text {Lor. }}^{-} \sim e^{-i \omega\left(t+r_{*}\right)}, \\
& e^{-i \omega t} \Phi_{\text {Lor. }}^{+} \sim e^{-i \omega\left(t-r_{*}\right)},
\end{aligned}
$$

where we have defined $r_{*}=\ln \left(r-r_{H}\right) /(4 \pi T)$ (the horizon now sits at $r_{*}=-\infty$ ). We tus note that the mode $e^{-i \omega t} \Phi_{\text {Lor. }}^{-}$corresponds to a wave moving towards the horizon (infalling), whereas $e^{-i \omega t} \Phi_{\text {Lor. }}^{+}$represents a wave moving away from the horizon (outgoing). It 
is clear that the most natural condition from the point of view of causality is the infalling one, and indeed this is the one that is associated to retarded correlators, which in turn are the causal ones.

In [44.45] it was shown how to calculate retarded Green's functions by imposing infalling boundary conditions at the black hole horizon. For black holes with non-degenerate horizons the retarded Green's functions are analytic in the upper half of the complexified frequency plane and have (an infinite series of) isolated poles in the lower half, yielding in general

$$
G_{\mathcal{R}}(\omega, \mathbf{k})=\sum_{n} \frac{\mathcal{R}_{n}(\mathbf{k})}{\omega-\omega_{n}(\mathbf{k})}+\tilde{G}(\omega, \mathbf{k})
$$

being $\tilde{G}(\omega, \mathbf{k})$ a part which is analytic in $\omega \in \mathbb{C}$ and $\mathcal{R}_{n}(\mathbf{k})$ are the residues of the Green's function. The series expansion (2.3.35) is convergent [46]. Poles $\omega=\omega_{n}(\mathbf{k})$ are the holographic quasinormal modes (QNM) of the black hole 4750 . Within the QNM spectrum, the ungapped modes play a special role, since they give the dominant contribution to the retarded Green's functions at low frequency and small momentum. Therefore they determine the hydrodynamic description of the system

$$
\lim _{\mathbf{k} \rightarrow 0} \omega_{n}(\mathbf{k})=0 \leftrightarrow \text { Hydrodynamic modes }
$$

QNMs are computed by solving a linear gravitational problem in General Relativity on top of a Black Brane background (see for instance [49]). In essence, we are taking the geometry corresponding to a global equilibrium situation and perturbing it slightly, meaning that the perturbations have small amplitude and thus Linear Response Theory is applicable. In general, however, this linear problem is not hermitean in nature and therefore the eigenvalues have complex values, which is to say, the most general form of $\omega_{n}(\mathbf{k})$ is

$$
\omega_{n}(\mathbf{k})=\Omega_{n}(\mathbf{k})-i \Gamma_{n}(\mathbf{k}),
$$

being $\Omega_{n}(\mathbf{k}), \Gamma_{n}(\mathbf{k}) \in \mathbb{R}$. Imaginary parts arise very naturally because we are considering the dynamics of a massless subsector interacting with a underlying thermalized soup and therefore the resulting energies are not constrained to be real. A similar picture arises when considering the quasiparticle spectrum in weakly interacting theories? the only (and crucial) difference being that all the imaginary parts are small in the weakly-coupled case. This is indeed a necessary condition for the mode to represent a quasiparticle

$$
\text { Quasiparticle } \rightarrow \lim _{\mathbf{k} \rightarrow 0} \frac{\Gamma_{n}(\mathbf{k})}{\Omega_{n}(\mathbf{k})}=0 .
$$

To see why, it is instructive to consider the temporal dependence of the $n$-th massless mode

$$
\phi_{n} \sim e^{-i \omega_{n}(\mathbf{k}) t}=e^{-\Gamma_{n}(\mathbf{k}) t} e^{-i \Omega_{n}(\mathbf{k}) t} .
$$

Clearly, $\operatorname{Im}\left(\omega_{n}(\mathbf{k})\right)$ quantifies the damping of the mode, i.e., how fast it decays with tim 8 , An estimate of the time that the system needs to return to thermal equilibrium after a

\footnotetext{
${ }^{7}$ Not every weakly-interacting system features quasiparticles. For instance, the Luttinger liquid does not present quasiparticle-type peaks in the spectral function.

${ }^{8}$ Notice in passing that the amplitude of any massive mode $\operatorname{Im}\left(\omega_{n}(\mathbf{k}=0)\right)=-M$ is exponentielly supressed. That is why hydrodynamic modes drive the leading response at large enought times.
} 
small perturbation is applied is $\Delta T_{\text {eq. }} \sim \frac{1}{\min .\left\{\Gamma_{n}\right\}}$, with $\left\{\Gamma_{n}\right\}=\left\{\Gamma_{n}(\mathbf{k}) \mid n \in \mathbb{Z}\right\}$. Observe now that if the limit appearing in (2.3.38) is satisfied the QNM is able to oscilate many times before decaying; for processes with a typical time scale of order $\Delta t<<1 / \Gamma_{n}$ the mode then behaves essentially as a particle, hence the name "qusiparticle". At strong coupling the quasiparticle picture is seriously jopardized and in general 2.3 .38 is not fulfilled in holography. However, for the examples that we will analyze in this work, (2.3.38) happen to hold.

It is shown in Appendix (11.3) that poles of the retarded correlators of the form 2.3.35) come in pairs, as long as the theory enjoys P-invariance. If $\omega_{n}$ is a pole in the complexified frequency plane, then $\tilde{\omega}_{n} \equiv-\omega_{n}^{*}$ is also a pole. The only possibility for the QNM no to be unpaired in the above sense is that $\omega_{n}=\tilde{\omega}_{n}$, so that it lies on the imaginary axis. We will see examples of this behaviour when studying diffusive modes in Chapter 8.2 .

It is also interesting to comment on the consequences of having $\Gamma_{n}(\mathbf{k})>0$ for some $n$. That would imply that the pole of the retarded Green's function lies on the upper half of the complex frequency plane. According to 2.3.39), the amplitude of such a mode would grow exponentially with time, signaling the perturbative instability of the system under consideration. This is the indication of a phase transition towards a stable phase in which the would-be tachyonic mode remains massless but stable. For example, the RN $\mathrm{BH}$ is unstable because the fluctuations of the scalar field in 1.2 .9 become tachyonic at certain critical temperature $T_{c}$ and the QNM spectrum of the stable phase below $T_{c}$, namely the holographic superfluid, contains a massless scalar pole. In general one can gain insight on the properties of the stable phase that the system will reach after undergoing the phase transition by looking at the properties of the mode that becomes tachyonic in the unstable phase. Imagine for instance that the tachyon has $\Gamma_{n}>0$ but independent of the momentum $\mathbf{k}$. This typically implies that the broken phase will be homogeneous. On the contrary, $\Gamma_{n}(\mathbf{k})>0$ only for finite values of $\mathbf{k}$ is suggesting that the broken phase will be non-homogeneous, i.e., it will break spacetime symmetries spontaneously. We will run into examples of this in Chapter 9.

The method we will be using to compute retarded correlators and the QNM spectrum is based on the one developed in [51]. For the shake of completeness, below we review it very briefly.

\section{Computing Green's functions and QNMs}

Consider a general bilinear bulk action for $M$ fields $\Phi^{I}, I=1, \ldots, M$

$$
S=\int \frac{d^{d} k}{(2 \pi)^{d}} \int d z\left[\Phi_{-k}^{\prime I} A_{I J}(k, z) \Phi_{k}^{\prime J}+\Phi_{-k}^{I} B_{I J}(k, z) \Phi_{k}^{\prime J}+\Phi_{-k}^{I} C_{I J}(k, z) \Phi_{k}^{J}\right],
$$

where we have Fourier transformed the transverse coordinates

$$
\Phi^{I}(x, z)=\int \frac{d^{d} k}{(2 \pi)^{d}} \Phi_{k}^{I}(k, z) e^{-i k x} ; \quad k \equiv k^{\mu}
$$

and matrices $A_{I J}, B_{I J}, C_{I J}$ depend only on the background and fulfill $A_{I J}(-k,, z)=$ $A_{I J}^{*}(k, z)$ (and similarly for $B_{I J}$ and $C_{I J}$ ). To avoid double counting of momenta, one split the momentum integration into "positive" $\left(k_{>}=(\omega>0, \mathbf{k})\right)$ and negative $k_{<}$. This yields

$$
S=\int \frac{d^{d-1} \mathbf{k}}{(2 \pi)^{d}} \int_{0}^{\infty} d \omega \int d z\left[2 \Phi_{-k}^{\prime I} A_{I J}^{H} \Phi_{k}^{\prime J}+\Phi_{-k}^{I} B_{I J} \Phi_{k}^{\prime J}+\Phi_{-k}^{I} B_{I J}^{\dagger} \Phi_{k}^{\prime J}+2 \Phi_{-k}^{I} C_{I J}^{H} \Phi_{k}^{J}\right]
$$


being $A^{H, A}=\left(A \pm A^{\dagger}\right) / 2$ the hermitean (anti-hermitean) part of the matrix. Upon using the $\mathrm{EOM}$ for $\Phi_{-k}^{I}$

$$
[E O M]_{\Phi_{-k}^{I}}=-2\left(A_{I J}^{H} \Phi_{k}^{\prime J}\right)^{\prime}+2 B_{I J}^{A} \Phi_{k}^{\prime J}+\left(2 C^{H}-B^{\prime \dagger}\right)_{I J} \Phi_{k}^{J}=0
$$

one can recast (2.3.42) as a boundary term

$$
\begin{aligned}
& S=\int \frac{d^{d-1} \mathbf{k}}{(2 \pi)^{d}} \int_{0}^{\infty} d \omega \int d z\left[\Phi_{-k}^{\prime I}[E O M]_{\Phi_{-k}^{I}}+\frac{d}{d z}\left[2 A_{I J}^{H} \Phi_{-k}^{I} \Phi_{k}^{\prime J}+B_{I J}^{\dagger} \Phi_{-k}^{I} \Phi_{k}^{J}\right]\right] \\
& \left.\stackrel{2.3 .43}{=} \int \frac{d^{d-1} \mathbf{k}}{(2 \pi)^{d}} \int_{0}^{\infty} d \omega \varphi_{-k}^{I} \mathcal{F}_{I J}(k, z) \varphi_{k}^{J}\right|_{z_{B}} ^{z_{H}}
\end{aligned}
$$

where we have implicitly express the fields in terms of their boundary values, i.e.

$$
\begin{aligned}
\Phi_{k}^{I}(z) & =F_{J}^{I}(k, z) \varphi_{k}^{I}, \\
\Phi_{-k}^{I}(z) & =\varphi_{-k}^{J} F_{J}^{\dagger}{ }^{I}(k, z),
\end{aligned}
$$

The quantity $F_{J}^{I}(k, z)$ is a solution matrix (the so-called bulk-to-boundary propagator), connecting the B.C. imposed at te boundary $z_{B}=0$, termed $\varphi_{k}^{I}$, with the interior. Clearly $F_{J}^{I}\left(k, z=z_{B}\right)=\delta_{J}^{I}$. If one is interested in numerical solutions, usually the boundary is placed at a cutoff $z_{\Lambda}$. From the above relations, one obtains $\mathcal{F}(k, z)=2 F^{\dagger} A^{H} F^{\prime}+F^{\dagger} B^{\dagger} F$. From here, applying the Minkowskian prescription of [44], one ends up with the following formula for the retarded Green's function

$$
G_{I J}^{R}(k)=-\lim _{z_{\Lambda} \rightarrow 0} \mathcal{F}_{I J}\left(k, z_{\Lambda}\right)=-\lim _{z_{\Lambda} \rightarrow 0}\left(2 A_{I K}^{H} F_{J}^{\prime K}+B_{I J}^{\dagger}\right) .
$$

If one needs to renormalize, the counterterms change the solution matrix $\mathcal{F}\left(k, z_{\Lambda}\right) \rightarrow$

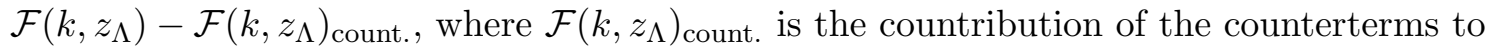
the solution matrix, which are responsible for rendering the Green's function finite.

Now, given a non-normalized matrix of solutions $H_{I J}(k, z)$, one can compute $F(k, z)=$ $H(k, z) H^{-1}\left(k, z_{\Lambda}\right)$. Poles of $G_{I J}^{R}$ can be found by imposing that relation 2.3.46) is actually ill-possed; since the dynamics are encoded in $F^{\prime}(k, z)$, one can look directly for inconsistencies associated with the bulk-to-boundary propagator. With this in mind, we observe that $F^{\prime}\left(k, z_{\Lambda}\right)$ cannot be defined if $H^{-1}\left(k, z_{\Lambda}\right)$ does not exists, i.e. if

$$
\operatorname{det}\left[H\left(k, z_{\Lambda}\right)\right]=0 ; \quad k=(\omega, \mathbf{k}) .
$$

Imposing (2.3.47), one arrives at (typically infinitely-many) solutions $\omega_{n}(\mathbf{k})$. Condition 2.3.47 can be seen to be enough to compute the dispersion relations of all the QNM spectrum of the holographic theory [51]. Among them, those QNMs satisfying $\omega_{n}(\mathbf{k}=$ $0)=0$ are identified with hydrodynamic modes and determine the late-time response of the system.

We will make use of the above construction repeatedly in this work. Further subtleties arise if the bulk action is gauge-invariant. The reason is that one then needs to come up with a prescription that ensures that the holographic correlators encode the response of the system to a gauge-invariant source, which is not a priori guaranteed. In Appendix 11.4 we present the method that we will follow in this work to treat gauge-invariant systems.

\subsection{Hydrodynamic Linear response}

Let us consider equation (2.3.24). We know that, whenever we have an scale in our physical system $\lambda_{m f p}$, the correlators at large distancies goes as $G_{R}^{\mathcal{O S}}\left(x-x^{\prime}\right) \sim e^{\frac{\left|\vec{x}-\vec{x}^{\prime}\right|}{\lambda_{m f p}}}$, 
so the range of the correlator is esentially $\lambda_{m f p}$ and the limits of integration in equation 2.3.24 have an effective range which is the mean free path.

Now, consider the situation in which the external source $J(\vec{x}, t)$ is very slowly varying, such that it can be considered to be constant on distances of order $\lambda_{m f p}$. Then, to zeroth-order in the spatial and temporal dependence of the source, we can write

$$
\delta\langle\mathcal{O}\rangle(x)=J(x) \int d^{4} x^{\prime} G_{R}^{\mathcal{O S}}\left(x-x^{\prime}\right)+(\text { terms of order } \mathrm{k})=\sigma J(x)+(\text { terms of order } \mathrm{k})
$$

We have taken $J\left(x^{\prime}\right)$ out of the integral with the form $J(x)$ because $J\left(x^{\prime}\right)$ takes the constant value $J(x)$ in the whole range $\left|\vec{x}-\vec{x}^{\prime}\right| \lesssim \lambda_{m f p}$.

We have turned the initial non-local expression for $\delta\langle\mathcal{O}\rangle$ into a local formula. The result 2.4.48 looks like the first term in a hydrodynamic expansion, with the constant $\sigma$ playing the role of the conductivity. Note that the existence of a scale $\lambda_{m f p}>0$ is essential to the argument.

The above construction establishes that there is a regime in which both Hydrodynamics and Linear Response Theory are good approaches to the description of the out-ofequilibrium physics of a given system, see Figure (2.1). As could have been anticipated, the regime in which both approximations overlap is the one in which the external sources have a very small amplitude and in addition they vary very little on scales of order $\lambda_{m f p}$. Hence, sticking to that region of the amplitude-momentum parameter space, we can make use of Kubo formulae to compute hydrodynamic transport coefficients. Moreover, as aforementioned, massless poles of retarded correlators computed using linear response give rise to hydrodynamic modes, such as the diffusive mode

$$
\omega(\mathbf{k})=-i D \mathbf{k}^{2} .
$$

This mode can be computed in holography using Linear Response Theory 52. On the other hand, linear-hydrodynamic techniques serve to calculate the first terms of the expansion in low energy and momentum of retarded correlators. 


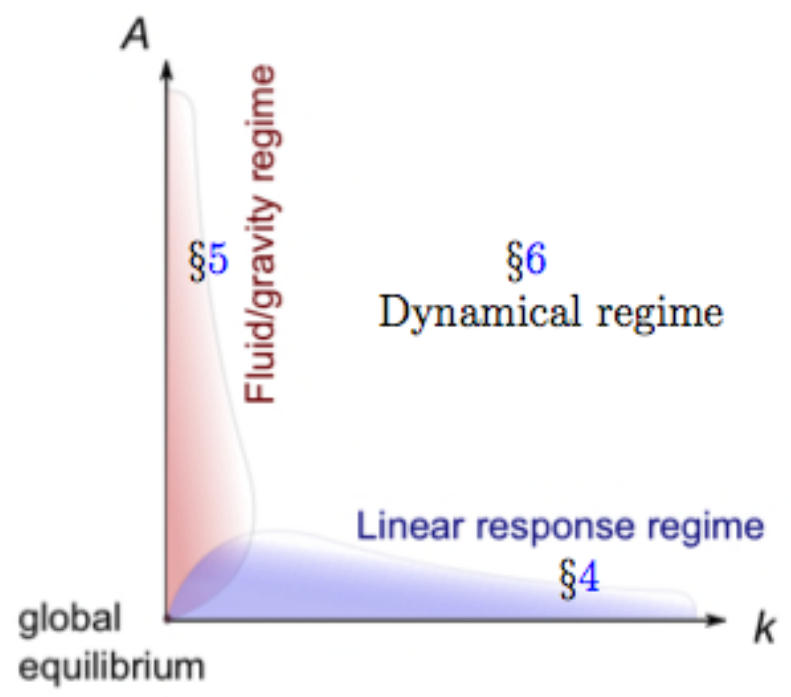

Figure 2.1: The regimes of validity of the two main approaches to out of equilibrium phenomena, in terms of the amplitude (A) and momentum (k) of the external perturbations. The figure has been taken from [1] 


\section{Chapter 3}

\section{Anomalies and Chiral transport phenomena at weak coupling}

Anomalies appear in the context of relativistic quantum field theories. In four dimensions chiral anomalies [53] involve triangle diagrams with either only vector currents or vector currents and the energy momentum tensor, in which case one speaks of a (mixed gauge-) gravitational anomaly [54. They are responsible for the breakdown of a classical symmetry due to quantum effects. If the symmetry is local anomalies impose severe restrictions on the structure and definition of gauge theories (for comprehensive reviews on anomalies see [55 57]). In the case of a symmetry generated by $T_{A}$, and considering only right-handed fermions, the presence of a chiral anomaly in vacuum is encoded in a non-vanishing $d_{A B C}=\frac{1}{2} \operatorname{tr}\left(T_{A}\left\{T_{B}, T_{C}\right\}\right)$. The corresponding parameter in the case of the gravitational anomaly is $b_{A}=\operatorname{tr}\left(T_{A}\right)$.

The history of anomalous transport coefficients starts with the study of high-barion density QCD, where it was shown that certain type of unusual effects may take place in the presence of topological defects due to anomalies $[58]^{1}$. In particular, an axial current would be generated in the direction of a magnetic field $B$ that is contained in a magnetic flux tube 63. This was latter called the Chiral Separation Effect(CSE)

$$
\overrightarrow{J_{5}}=\sigma_{C S E} \vec{B}
$$

with $\sigma_{C S E}=\frac{e N_{c}}{2 \pi^{2}} \mu$. A similar effect was proposed when studying the Quark-Gluon Plasma (QGP) generated in heavy-ion collisions [64 66]. This is called the Chiral Magnetic Effect (CME) 67,68

$$
\vec{J}=\sigma_{C M E} \vec{B}
$$

being $\sigma_{C M E}=\frac{e^{2} N_{c}}{2 \pi^{2}} \mu_{5}$, and leads to the appearance of a vector current in the direction of the external magnetic field if an axial chemical potential $\mu_{5}$ is present ${ }^{2}$

There is another effect obtained firstly by holographic methods, which represents the generation of an axial current due to an axial chemical potential and axial magnetic field

$$
\vec{J}_{5}=\sigma_{55} \vec{B}_{5}
$$

\footnotetext{
${ }^{1}$ Remarkable enough, the existence of anomalous transport was actually predicted more than twenty years before by Vilenkin 5962

${ }^{2}$ It has been argued in 64 that an imbalance between right- and left-handed quarks is generated locally due to the existence of sphalerons. This imbalance could be modeled by an axial chemical potential after equilibrium is reached.
} 
with $\sigma_{55}=\frac{N_{c}}{2 \pi^{2}} \mu_{5}$. Experimentally, this effect is less interesting, because axial magnetic fields do not exist in nature. However, recent studies on Weyl semimetals indicate that both $\mu_{5}$ and $\vec{B}_{5}$ could be effectively seen as features of the Dirac cones in these materials. After these developments it was also shown that, in addition, a vortex can also generate a current in the direction of vorticity

$$
\begin{aligned}
\vec{J}_{5} & =\sigma_{A}^{\mathcal{V}} \vec{\omega}, \\
\vec{J} & =\sigma_{V}^{\mathcal{V}} \vec{\omega} .
\end{aligned}
$$

Here $\sigma_{\{A, V\}}^{\mathcal{V}}$ is called Chiral Vortical Conductivity (CVC) generically and $\vec{\omega}$ is the vorticity vector. Soon after a consistent holographic calculations of the CVC and CMC where performed via Kubo formulae in holography [69], it was shown by direct calculation of the CVC for a gas of free fermions at finite temperature that the existing literature had been missing a term proportional to the temperature squared 70

$$
\sigma_{A}^{\mathcal{V}}=\frac{\mu_{5}^{2}+\mu^{2}}{4 \pi^{2}}+\frac{T^{2}}{12}
$$

It was also conjectured in 70 that such a new term is actually related to the Mixed Gauge-Gravitational anomaly [54]. For a exaustive discussion on chiral conductivities see 71 .

\section{A few remarks on anomalies}

Here we provide here a very short introduction to anomalies. Let us assume that we have a QFT in the presence of a $U(1)$ gauge field $A_{\mu}$ and a metric $g_{\mu \nu}$ background fields. This serves to weakly gauge the theory. Our generating functional reads

$$
W[A, g]=-i \ln Z[A, g]
$$

being $Z[A, g]$ the partition function. A generic variation of $W[A, g]$ is

$$
\delta W[A, g]=\int d^{4} x \sqrt{-g}\left[\delta A_{\mu} \tilde{J}^{\mu}+\frac{1}{2} \delta g_{\mu \nu} \tilde{T}^{\mu \nu}\right],
$$

where $\tilde{J}^{\mu}$ is the current and $\tilde{T}^{\mu \nu}$ the energy momentum tensor. After weakly gauging the theory, we have $U(1)$ gauge and also diffeomorphisms. We call " $s$ " a transformation of this sort. After integrating by parts, we get

$$
\delta_{s} W[A, g]=-\int d^{4} x \sqrt{-g}\left[\epsilon \nabla_{\mu} \tilde{J}^{\mu}+\xi_{\nu}\left(\nabla_{\mu} \tilde{T}^{\mu \nu}-F^{\nu \lambda} \tilde{J}_{\lambda}+A^{\nu} \nabla_{\lambda} \tilde{J}^{\lambda}\right)\right],
$$

where $\epsilon(x)$ is the parameter associated to the gauge transformation of $A_{\mu}$ and $\delta x^{\mu}=\xi^{\mu}$ is a coordinate transformation.

When an anomaly is present $\delta_{s} W[A, g] \neq 0$. The anomaly can be parametrized by the anomaly polynomial $\mathcal{P}$, that takes the form

$$
\mathcal{P}=a F \wedge F \wedge F+b F \wedge R_{\nu}^{\mu} \wedge R_{\mu}^{\nu} .
$$

In other words, we have allowed for a $U(1)^{3}$ anomaly plus a Mixed Gauge Graviational Anomaly. We will comment on parameters $a$ and $b$ later on. Due to Bianchi identities, 
it is easy to see that $d \mathcal{P}=0$, so there exists a five-form $I_{C S}$ such that $\mathcal{P}=d I_{C S}$ whose explicit expression is 3

$$
I_{C S}=a A \wedge F \wedge F+b A \wedge R_{\nu}^{\mu} \wedge R_{\mu}^{\nu},
$$

satisfying $\delta_{s} I_{C S}=d G_{s}$, with

$$
G_{s}=\epsilon\left(a F \wedge F+b R_{\nu}^{\mu} \wedge R_{\mu}^{\nu}\right)
$$

It turns out that one can relate the gauge transformation of $W[A, g]$ to $G_{s}$ as

$$
\delta_{s} W[A, g]=-\int G_{s}
$$

which leads to the anomalous Ward identities

$$
\begin{aligned}
\nabla_{\mu} \tilde{J}^{\mu} & =\frac{1}{4} \epsilon^{\mu \nu \rho \lambda}\left[a F_{\mu \nu} F_{\rho \lambda}+b R_{\beta \mu \nu}^{\alpha} R_{\alpha \rho \lambda}^{\beta}\right], \\
\nabla_{\nu} \tilde{T}^{\mu \nu} & =F^{\mu \nu} J_{\nu}-\frac{1}{4} A^{\mu} \epsilon^{\nu \rho \lambda \tau}\left[a F_{\nu \rho} F_{\lambda \tau}+b R_{\beta \nu \rho}^{\alpha} R_{\alpha \lambda \tau}^{\beta}\right]
\end{aligned}
$$

Up to now, the discussion concerned consistent currents, which involve variations of the generating functional and take their name due to the fact that they obey the Wess-Zumino consistency condition [73]. Now, that condition states that $-\delta \int G_{s}=\delta \delta_{s} W[A, g]=$ $\delta_{s} \delta W[A, g]$. Expanding this expression we get that

$$
-\delta \int G_{s}=\int d^{4} x \sqrt{-g}\left[\delta A_{\mu} \delta_{s} \tilde{J}^{\mu}+\frac{1}{2} \delta g_{\mu \nu} \delta_{s} \tilde{T}^{\mu \nu}\right] .
$$

By the form of (3.0.11), it is clear that $\delta \int G_{s}$ will be a non-vanishing and non-covariant functional of the external fields (the Bardeen-Zumino polynomials 72$]$ ). This implies that the consistent current and energy-momentum tensor do not transform covariantly. To fix this, one can define new operators

$$
\begin{aligned}
J^{\mu} & =\tilde{J}^{\mu}+P^{\mu}, \\
T^{\mu \nu} & =\tilde{T}^{\mu \nu}+K^{\mu \nu},
\end{aligned}
$$

such that the so-called covariant current and energy-momentum tensor $J^{\mu}$ and $T^{\mu \nu}$ transform covariantly under $\delta_{s}$. The Ward identities for the covariant objects are

$$
\begin{aligned}
\nabla_{\mu} J^{\mu} & =\frac{1}{4} \epsilon^{\mu \nu \rho \lambda}\left[a F_{\mu \nu} F_{\rho \lambda}+b R_{\beta \mu \nu}^{\alpha} R_{\alpha \rho \lambda}^{\beta}\right], \\
\nabla_{\nu} T^{\mu \nu} & =F^{\mu \nu} J_{\nu}+\frac{b}{2} \nabla_{\nu}\left[\epsilon^{\alpha \beta \rho \lambda} F_{\alpha \beta} R_{\rho \lambda}^{\mu \nu}\right] .
\end{aligned}
$$

The covariant definition of the operators cannot be derived for a generating functional (notice that the transformation (3.0.16) is performed directly on the operators) and moreover do not satisfy the Wess-Zumino consistency condition [55].

For future applications, it is important to remark here that the chiral transport coefficients are related to the response of the covariant operators.

In the following we firstly address some subtleties related to the definition of the chemical potential and then we move to the presentation of the computations of the anomalous transport coefficients at weak coupling, following the works 67, 70. We leave the strong coupling, large-N calculation for Chapter 5 .

\footnotetext{
${ }^{3}$ Notice that $I_{C S}$ is defined up to an arbitrary total derivative. In models with axial and vector symmetries and a AVV anomaly, the total derivative can be used to move the anomaly from the axial sector to the vector sector and vice versa. This corresponds to the implementation of the well-known Bardeen counterterm 72. In the case at hand such a counterterm cannot be defined (it vanishes trivially) for we have simply a AAA anomaly.
} 


\subsection{Chemical potential for anomalous symmetries}

Let us start by pointing out some subtleties related to the definition of a chemical potential for anomalous symmetries. In the grand canonical ensemble, the chemical potential $\mu$ enters frequently as a source of the conserved charge operator $\mathcal{Q}$, which generates a (global) symmetry of the system. In QFT a thermal expectation value admits a path integral representation

$$
\langle\mathcal{O}\rangle=\int \mathcal{D}[\phi(x)] \mathcal{O}[\phi(x)] e^{-S_{E}},
$$

where $S_{E}$ is the euclidean action and the boundary conditions are supposed to be periodic/antiperiodic in time $\phi(t-i \beta)= \pm e^{\mu \beta} \phi(t)$ (plus sign for bosons and minus sign for fermions). To simplify the mentioned boundary conditions, one could instead redefine

$$
\tilde{\phi}(x)=e^{i \mu t} \phi(x)
$$

and consider $\tilde{\phi}(t-i \beta)= \pm \tilde{\phi}(t)$. This transformation is equivalent to having the timederivatives shifted as $i \partial_{0} \rightarrow i \partial_{0}+\mu$, or, in an operator form, to having the modified Hamiltonian

$$
H \rightarrow H-\mu Q
$$

These two formalisms are equivalent as long as the charge generates a global symmetry, i.e. $[H, Q]=0$. Indeed, we can think of transformation $(3.1 .22)$ as introducing a fiducial gauge field $A_{\mu}$ coupled to the current in the Lagrangian, namely $\Delta \mathcal{L} \sim \int d^{4} x A_{\mu} j^{\mu}$ and then assume $A_{0} \equiv \mu$. Notice that, after introducing $A_{\mu}$, the system enjoys full gauge invariance and the relation between formalism (A) and (B) is just a gauge transformation

$$
A_{0} \rightarrow A_{0}+\partial_{0} \chi ; \quad \chi=-\mu t
$$

The above transformation induces the twist on the boundary conditions of $\phi(x)$ (in imaginary time direction) and removes the coupling $\mu Q$ from the Hamiltonian.

However, in the case at hand we have to keep in mind that the symmetry is anomalous and hence $[H, Q] \neq 0$ in general

$$
[H, Q] \propto C_{1} \int \operatorname{Tr}(F \wedge F)+C_{2} \int \operatorname{Tr}(R \wedge R),
$$

meaning that the charge decays due to instanton or sphaleron processes when subjected to time evolution. In other words, a eigenstate of the charge at initial time $t_{i}$ will cease to have a well-defined charge at times $t_{i}+\Delta t$.

The above argument favoures formalism (B): we start with some prepared state at $t_{i}$ and let it evolve with the microscopic Hamiltonian $H$. Let us start with (B) and try to recover (A). To that end, we consider a gauge transformation, which is non-trivial now due to the anomay

$$
S[A+\partial \chi]=S[A]+\int d^{4} x \chi \epsilon^{\mu \nu \rho \lambda}\left(C_{1} F_{\mu \nu} F_{\rho \lambda}+C_{2} R_{\beta \mu \nu}^{\alpha} R_{\alpha \rho \lambda}^{\beta}\right) .
$$

In order to "remove" the anomaly, we introduce an auxiliary non-dynamical axion field $\Theta(x)$ that transforms as $\Theta \rightarrow \Theta-\chi$, and the vertex

$$
S_{\Theta}[A, \Theta]=\int d^{4} x \Theta \epsilon^{\mu \nu \rho \lambda}\left(C_{1} F_{\mu \nu} F_{\rho \lambda}+C_{2} R_{\beta \mu \nu}^{\alpha} R_{\alpha \rho \lambda}^{\beta}\right) .
$$


With this ingredients, we see that the total action

$$
S_{\mathrm{TOT}}[A, \Theta]=S[A]+S[A, \Theta]
$$

is gauge invariant. Notice that the resulting consistent current $\delta S_{\mathrm{TOT}} / \delta A_{\mu}$ is still anomalous, even though it should be possible to define an anomaly-free current from the action (3.1.27). In fact, the introduction of the axion $\Theta(x)$ is at the core of the methods used over the years when attempting to defining consistently an anomalous theory. We observe now that we recover formalism (B) by assuming $A_{0}=0$. A gauge transformation with $\chi=\mu t$ implies $\Theta=-\mu t$, and formalism (A) can be obtained from the field configuration $A_{0}=\mu$. The axion contribution to $S_{\mathrm{TOT}}$ induces a term in the current $j_{\Theta}^{\mu}=4 C_{1} \epsilon^{\mu \nu \rho \lambda} \partial_{\nu} \Theta F_{\rho \lambda}$, which evaluated for $\Theta=-\mu t$ implies 4

$$
j_{\Theta}^{i}=4 C_{1} \mu B_{i}
$$

At this point is it important to remark that this is not the Chiral Magnetic Effect, it is only a contribution to the consistent current that arises when we try to go from formalism (B) to (A) in a gauge-invariant way. As we will see, Chiral Magnetic and Vortical effects are non-trivial results of dynamical one-loop calculations. We conclude that the actual Hamiltonian in formalism (A) is not $H-\mu Q$, but

$$
H-\mu\left(Q+4 \int d^{3} x\left(C_{1} \epsilon^{0 i j k} A_{i} \partial_{j} A_{k}+C_{2} K^{0}\right)\right)
$$

where $K^{0}$ is the zeroth-component of the gravitational Chern-Simons current fulfilling $\partial_{\mu} K^{\mu}=\frac{1}{4} \epsilon^{\mu \nu \rho \lambda} R_{\beta \mu \nu}^{\alpha} R_{\alpha \rho \lambda}^{\beta}$, namely $K^{\mu}=\epsilon^{\mu \nu \rho \lambda} \Gamma_{\beta \nu}^{\alpha}\left(\partial_{\rho} \Gamma_{\alpha \lambda}^{\beta}+\frac{2}{3} \Gamma_{\rho \sigma}^{\beta} \Gamma_{\alpha \lambda}^{\sigma}\right)$.

\subsection{Anomalous Hydrodynamics}

We left the discussion on Hydrodynamics in Section 2.2. Here we extend it up to first order in momenta, including anomalous contributions. Let us generalize and reformulate a little bit equations (3.0.18), to arrive at the following form

$$
\begin{array}{r}
D_{\mu} T^{\mu \nu}=F_{a \nu}^{\mu} J_{a}^{\nu}+2 \lambda_{a} D_{\nu}\left[\epsilon^{\rho \sigma \alpha \beta} F_{a \rho \sigma} R^{\mu \nu}{ }_{\alpha \beta}\right], \\
\left(D_{\mu} J^{\mu}\right)_{a}=\epsilon^{\mu \nu \rho \lambda}\left(3 \kappa_{a b c} F_{\mu \nu}^{b} F_{\rho \lambda}^{c}+\lambda_{a} R_{\beta \mu \nu}^{\alpha} R_{\alpha \rho \lambda}^{\beta}\right),
\end{array}
$$

where $\bar{\kappa}_{a b c}=\frac{d_{a b c}}{96 \pi^{2}}$ and $\bar{\lambda}_{a}=\frac{b_{a}}{768 \pi^{2}} ; d_{a b c}$ and $b_{a}$ being defined in the first paragraph of Section 3 .

In order to construct the first order constitutive relations, we have to consider the most general expansion in terms of one derivative of the velocity, temperature and chemical potential, in the background of a gauge field $A_{\mu}$ and a metric $g_{\mu \nu}$. First of all, we expand the derivative of the fluid velocity

$$
D^{\nu} u^{\mu}=-a^{\mu} u^{\nu}+\sigma^{\mu \nu}+\frac{1}{2} \omega^{\mu \nu}+\frac{1}{3} \theta P^{\mu \nu},
$$

\footnotetext{
${ }^{4} e^{0 i j k}=-\epsilon_{i j k}$ in Minkownski space.
} 


\begin{tabular}{|c|c|c|c|c|c|c|c|c|}
\hline & $\theta$ & $D_{\mu} \bar{\mu}$ & $D_{\mu} T$ & $a^{\mu}$ & $E_{a}^{\mu}$ & $B_{a}^{\mu}$ & $\omega^{\mu}$ & $\sigma^{\mu \nu}$ \\
\hline$C$ & + & - & + & + & - & - & + & + \\
$P$ & + & - & - & - & - & + & + & + \\
\hline
\end{tabular}

Table 3.1: Properties of first order quantities under CP.

where $a^{\mu}, \sigma^{\mu \nu}, \omega^{\mu \nu}$ and $\theta$ are called acceleration, shear tensor, vorticity tensor and expansion respectively. Their definition is the following

$$
\begin{aligned}
\theta & \equiv D_{\mu} u^{\mu}=P^{\mu \nu} D_{\mu} u_{\nu}, \\
a^{\mu} & \equiv u^{\nu} D_{\nu} u^{\mu}, \\
\sigma^{\mu \nu} & \equiv D^{(\mu} u^{\nu)}+a^{(\mu} u^{\nu)}-\frac{1}{3} \theta P^{\mu \nu} \equiv D^{<\mu} u^{\nu>}, \\
\omega^{\mu \nu} & \equiv 2 D^{[\mu} u^{\nu]}+u^{[\mu} a^{\nu]}=2 P^{\alpha \beta} P^{\nu \beta} D_{[\alpha} u_{\beta]} .
\end{aligned}
$$

The above definition implies that the acceleration is transverse and the shear and vorticity tensors are both transverse and traceless. Moreover, from the vorticity tensor one can also construct the vorticity vector

$$
\omega^{\mu}=\frac{1}{2} \epsilon^{\mu \nu \rho \lambda} u_{\nu} \omega_{\rho \lambda}
$$

From the metric and the gauge field we can define

$$
\begin{aligned}
F_{\mu \nu}^{a} & =\partial_{\mu} A_{\nu}^{a}-\partial_{\nu} A_{\mu}^{a}+g f^{a b c} A_{\mu}^{b} A_{\nu}^{c}, \\
E_{\mu}^{a} & =F_{\mu \nu}^{a} u^{\nu}, \\
B^{a \mu} & =\frac{1}{2} \epsilon^{\mu \nu \rho \lambda} u_{\nu} F_{\rho \lambda}^{a},
\end{aligned}
$$

which correspond to field-strenght, electric and magnetic fields, respectively. By means of thermodynamic charge and energy conservation, it turns out that we can define just one independent scalar and five independent vectors, that are usually taken to be

$$
\theta ; \quad P^{\mu \nu} D_{\nu} \bar{\mu} ; \quad P^{\mu \nu} D_{\nu} T ; \quad \omega^{\mu} ; \quad E_{\mu}^{a} ; \quad B^{a \mu},
$$

where $\bar{\mu}=\frac{\mu}{T}$.

\subsubsection{Transformations under Charge Conjugation(C) and $\operatorname{Parity}(\mathrm{P})$}

In this section we will follow closely the analysis of 74 . We find that under $(C, P)$ the different quantities behave as

$$
\begin{aligned}
& (C, P) u^{\mu}=(+,-), \quad(C, P) g_{\mu \nu}=(+,+), \quad(C, P) \epsilon^{\mu \nu \rho \lambda}=(+,-) \\
& (C, P) D_{\mu}=(+,-), \quad(C, P) J^{\mu}=(-,-), \quad(C, P) A_{\mu}^{a}=(-,-), \quad(C, P) S^{\mu}=(+,-) .
\end{aligned}
$$

which implies $(C, P) n_{a}=(-,-),(C, P) \mu_{a}=(-,-),(C, P) s=(+,+)$ and $(C, P) T=$ $(+,+)$. The rest of the transformations under $(C, P)$ are summarized in Table 3.1. Assuming that the transport coefficients are functions of $f(\bar{\mu}, \kappa, \lambda)$ (the transformation of $T$ 
under $(C, P)$ is trivial and hence will not be considered in the following analysis), we can classify the possible functions depending on its transformation under $(C, P)$

$$
\begin{array}{ll}
(+,+): & f(\bar{\mu}, \kappa, \lambda)=g\left(\bar{\mu}^{2}, \kappa^{2}, \lambda^{2}, \kappa \lambda\right) \\
(-,+): & f(\bar{\mu}, \kappa, \lambda)=\bar{\mu} g\left(\bar{\mu}^{2}, \kappa^{2}, \lambda^{2}, \kappa \lambda\right) \\
(-,-): & f(\bar{\mu}, \kappa, \lambda)=\kappa g_{1}\left(\bar{\mu}^{2}, \kappa^{2}, \lambda^{2}, \kappa \lambda\right)+\lambda g_{2}\left(\bar{\mu}^{2}, \kappa^{2}, \lambda^{2}, \kappa \lambda\right) \\
(+,-): & f(\bar{\mu}, \kappa, \lambda)=\bar{\mu} \kappa g_{1}\left(\bar{\mu}^{2}, \kappa^{2}, \lambda^{2}, \kappa \lambda\right)+\bar{\mu} \lambda g_{2}\left(\bar{\mu}^{2}, \kappa^{2}, \lambda^{2}, \kappa \lambda\right)
\end{array}
$$

We can now identify the anomalous transport coefficients for they transform oddly under parity, namely, their transformation under $(C, P)$ is $( \pm,-)$. The most general nonvanishing first order corrections to ideal Hydrodynamics is therefore

$$
\begin{aligned}
f_{\mathcal{P}} & =-\zeta \theta, \\
\tau^{\mu \nu} & =-2 \eta \sigma^{\mu \nu}, \\
q^{\alpha} & =\xi_{a}^{1} E_{a}^{\mu}+\xi_{a}^{2} P^{\alpha \nu} D_{\nu} \bar{\mu}_{a}+\xi^{3} P^{\alpha \nu} D_{\nu} T, \\
\tilde{q}^{\alpha} & =\sigma_{a}^{(\epsilon, B)} B_{a}^{\mu}+\sigma^{(\epsilon, V)} \omega^{\mu}, \\
j_{a}^{\alpha} & =\Omega_{a b}^{1} E_{b}^{\mu}+\Omega_{a b}^{2} P^{\alpha \nu} D_{\nu} \bar{\mu}_{b}+\Omega_{a}^{3} P^{\alpha \nu} D_{\nu} T, \\
\tilde{j}_{a}^{\alpha} & =\sigma_{a b}^{B} B_{b}^{\mu}+\sigma_{a}^{V} \omega^{\mu} .
\end{aligned}
$$

The tilded quantities refer to anomalous contributions. In a frame-invariant language

$$
\begin{aligned}
l_{a}^{\mu} & =-\Sigma_{a b} E_{b}^{\mu}-\bar{\Sigma}_{a b} P^{\alpha \nu} D_{\nu} \bar{\mu}_{b}+\xi_{a b}^{B} B_{b}^{\mu}+\xi_{a}^{V} \omega^{\mu}+\chi_{a}^{T} P^{\alpha \nu} D_{\nu} T \\
f & =-\xi \theta
\end{aligned}
$$

with

$$
\begin{array}{rlrl}
\Sigma_{a b} & =\left(\Omega_{a b}^{1}-\frac{n_{a}}{\epsilon+P} \xi_{b}^{1}\right) ; & \bar{\Sigma}_{a b}=\left(\Omega_{a b}^{2}-\frac{n_{a}}{\epsilon+P} \xi_{b}^{2}\right) ; \\
\xi_{a b}^{B}=\left(\sigma_{a b}^{B}-\frac{n_{a}}{\epsilon+p} \sigma_{b}^{(\epsilon, B)}\right) ; & \xi_{a}^{V}=\left(\sigma_{a}^{V}-\frac{n_{a}}{\epsilon+p} \sigma^{(\epsilon, V)}\right) ; \\
\chi_{a}^{T}=\left(\Omega_{a}^{3}-\frac{n_{a}}{\epsilon+p} \xi^{3}\right) . & &
\end{array}
$$

\subsubsection{Constraints from positivity of the entropy current}

One can further constraint the coefficients imposing the positivity of the entropy current

$$
D_{\mu} S^{\mu} \geq 0
$$

The consequences of the above condition were studied by Son and Surowka 75 . We do not enter into details here and refer the reader to the original reference for details. The outcome of the calculation is that positivity of $D_{\mu} S^{\mu}$ forces the most general constitutive relations in the Landau frame for the energy-momentum tensor and the covariant currents to be

$$
\begin{aligned}
T^{\mu \nu} & =\epsilon u^{\mu} u^{\nu}+(p-\xi \theta) P^{\mu \nu}-2 \eta \sigma^{\mu \nu}, \\
J_{a}^{\mu} & =n_{a} u^{\mu}+\Sigma_{a b}\left(E_{b}^{\mu}-T P^{\mu \alpha} D_{\alpha} \bar{\mu}_{b}\right)+\xi_{a b}^{B} B_{b}^{\mu}+\xi_{a}^{V} \omega^{\mu},
\end{aligned}
$$


with the following constraints on the transport coefficients

$$
\begin{aligned}
& \eta \geq 0, \quad \xi \geq 0, \quad \Sigma_{a b} \geq 0, \quad \bar{\Sigma}_{a b}=T \Sigma_{a b}, \quad \chi_{a}^{T}=0, \\
& \xi_{a b}^{B}=24 \bar{\kappa}_{a b c} \mu_{c}-\frac{n_{a}}{\epsilon+p}\left(12 \bar{\kappa}_{a b c} \mu_{c} \mu_{d}+\beta_{b} T^{2}\right), \\
& \xi_{a}^{V}=12 \bar{\kappa}_{a b c} \mu_{b} \mu_{c}+\beta_{a} T^{2}-\frac{n_{a}}{\epsilon+p}\left(8 \bar{\kappa}_{a b c} \mu_{b} \mu_{c} \mu_{d}+2 \beta_{b} \mu_{b} T^{2}+\gamma T^{3}\right) .
\end{aligned}
$$

We observe that the anomalous transport coefficients are fully determined by the anomaly coefficients, up to the quantities $\beta_{a}$ and $\gamma$, that enter the equations as integration constants and are not fixed by the method. However, $\gamma$-terms break CPT invariance [76. The constant $\beta_{a}$ is fixed by the Mixed Graviational anomaly, as we will see later on in Chapter 5. There are other strategies to obtain the chiral conductivities 3.2 .49 without imposing (3.2.45) (see for instance [76 80]).

In order to obtain the anomalous hydrodynamic expansion for a CFT using holography, one can make use of the Fluid/Gravity correspondence techniques commented on in Section 2.2.2. Here we will not elaborate on that construction. The interested reader can consult 81 for the computation up to first order in momenta, or [82, 83 for the construction up to second order. Other interesting holographic approaches can be found in 84, 85.

\subsection{Anomalies and Kubo formulae}

It is possible to use Kubo formulae in order to compute the anomalous conductivities. The Landau frame, defined in Section 2.2.1, turns out not to be the most convenient one in this case, due to the fact that it involves a definition of the superfluid velocity that absorbs the anomaly contributions to the energy current, so that there are no anomalous terms in its constitutive relations, see equation (3.2.46). In the following we go to the so called laboratory-frame, in which the constitutive relations to $\mathcal{O}(k)$, particularizing for a single $U(1)$ symmetry, read

$$
\begin{aligned}
T^{\mu \nu} & =\epsilon u^{\mu} u^{\nu}+(p-\xi \theta) P^{\mu \nu}-\eta \sigma^{\mu \nu}+2 \tilde{q}^{(\mu} u^{\nu)}, \\
J^{\mu} & =n u^{\mu}+\Sigma\left(E^{\mu}-T P^{\mu \alpha} D_{\alpha} \bar{\mu}\right)+\sigma^{B} B^{\mu}+\sigma^{V} \omega^{\mu},
\end{aligned}
$$

with $\tilde{q}^{\mu}$ given by equation (3.2.39). Since we know the anomalous part of the constitutive relations (we pick the $x$-direction for simplicity)

$$
\begin{aligned}
\delta T^{0 x} & =\sigma_{B}^{\epsilon} B_{x}+\sigma_{V}^{\epsilon} \omega_{x}, \\
\delta J^{x} & =\sigma_{B} B_{x}+\sigma_{V} \omega_{x},
\end{aligned}
$$

we can take the variation of these one point functions with respect to the external perturbative gauge fields and metric in momentum space $\delta A_{\mu}(x)=\delta A_{\mu}(\omega, \vec{k}) e^{-i \omega t+i \vec{k} \cdot x}$; $\delta g_{\mu \nu}(x)=\delta g_{\mu \nu}(\omega, \vec{k}) e^{-i \omega t+i \vec{k} \cdot x}$ in order to get

$$
\begin{aligned}
\sigma_{B} & =\lim _{k_{z} \rightarrow 0} \frac{i}{k_{z}}\left\langle J^{x} J^{y}\right\rangle_{\mathcal{R}}\left(k_{z}, \omega=0\right), \\
\sigma_{V} & =\lim _{k_{z} \rightarrow 0} \frac{i}{k_{z}}\left\langle J^{x} T^{0 y}\right\rangle_{\mathcal{R}}\left(k_{z}, \omega=0\right), \\
\sigma_{B}^{\epsilon} & =\lim _{k_{z} \rightarrow 0} \frac{i}{k_{z}}\left\langle T^{0 y} J^{x}\right\rangle_{\mathcal{R}}\left(k_{z}, \omega=0\right), \\
\sigma_{V}^{\epsilon} & =\lim _{k_{z} \rightarrow 0} \frac{i}{k_{z}}\left\langle T^{0 y} T^{0 x}\right\rangle_{\mathcal{R}}\left(k_{z}, \omega=0\right),
\end{aligned}
$$


where $\mathcal{R}$ stands for "retarded" and we have chosen the momentum pointing in the $z$ direction taking advantage of rotation invariance. Notice that all the correlators are computed at zero frequency. This means that there is no dynamics associated to such correlators or, in other words, that we could have just used a euclidean compactification of the time coordinate in order to compute them. But the latter statement is equivalent to calculating the correlators at equilibrium. Indeed, it can be seen that the anomalous transport coefficients, despite the fact that they are seen at first order in momentum, are equilibrium quantities 77,86 . This fact also suggest that such coefficients are nondissipative. In fact, it is possible to show that chiral conductivities are related to the anti-Hermitian part of the retarded correlators, whereas usual transport coefficients sit in the Hermitian part. The rate at which an external source $f_{I}$ works on the system is given in terms of the spectral function of the operator $\mathcal{O}^{I}$ (that couples to $f_{I}$ ) by

$$
\frac{d W^{I}}{d t}=\frac{\omega}{2} f_{I}(-\omega) \rho^{I J}(\omega) f_{J}(\omega)
$$

where $\rho^{i j}=\frac{-i}{2}\left(G_{\mathcal{R}}-G_{\mathcal{R}}^{+}\right)$is the spectral function, corresponding essentially to the Hermitian part of the retarded Green's function $G_{\mathcal{R}}$. We thus observe that usual transport coefficients are dissipative, whereas anomalous transport coefficients are not. As we will see, this is no longer the case necessarily when considering chiral transport in superfluids.

Equations (3.3.53)- 3.3 .56$)$ play a major role in this work, since we are going to make use of them very frequently to compute chiral transport coefficients. A pertinent remark is however in order. Equations (3.3.52 represent the response of covariant current and energy-momentum tensor. Actually, those equations have to be understood quantummechanically, i.e. as an expression of one-point functions in terms of external sources. Kubo formulae (3.3.53)-(3.3.56) are obtaining by varying the one point function with respect to the external source, which is of course equivalent to perform a variation of the generating functional. This implies that for instance (3.3.53) corresponds actually to a correlator involving one covariant and one consistent current, namely $\sigma_{B} \sim\left\langle J^{x} \tilde{J}^{y}\right\rangle_{\mathcal{R}}$ (in the notation of Section 3). In the rest of this work we will omit the $\sim$ on top of the covariant current for simplicity, but one has to keep in mind its precise definition.

If we are now to relate the anomalous transport coefficients in the Laboratory frame with the corresponding ones in the Landau frame, we have to perform the following velocity transformation

$$
u^{\mu} \rightarrow u^{\mu}-\frac{q^{\mu}}{\epsilon+p} .
$$

In other words, the coefficients termed $\sigma$ in equations 3.2 .43 are indeed the anomalous transport coefficients in the laboratory frame, and the desired transformation between Laboratory and Landau frames is no other but the one prescribed by these precise equations, i.e,

$$
\begin{aligned}
& \xi_{B}=\left.\lim _{k_{c} \rightarrow 0} \frac{i}{2 k_{c}} \sum_{a, b} \epsilon_{a b c}\left(\left\langle J^{a} J^{b}\right\rangle-\frac{n}{\epsilon+P}\left\langle T^{0 a} J^{b}\right\rangle\right)\right|_{\omega=0, A_{0}=0}, \\
& \xi_{V}=\left.\lim _{k_{c} \rightarrow 0} \frac{i}{2 k_{c}} \sum_{a, b} \epsilon_{a b c}\left(\left\langle J^{a} T^{0 b}\right\rangle-\frac{n}{\epsilon+P}\left\langle T^{0 a} T^{0 b}\right\rangle\right)\right|_{\omega=0} .
\end{aligned}
$$




\subsubsection{Anomalous conductivities at weak coupling from Kubo Formulae}

It turns out that it is possible to evaluate the Kubo formulae $(3.3 .53)-(3.3 .56)$ for $N$ non-interacting and right-handed fermions $\Psi^{f} ; f=1, \ldots N$ transforming under a global symmetry group generated by $\left(T_{A}\right)^{f}$. The Chiral Magnetic Conductivity was computed in this way in 67 for the first time. The calculation reduces to the evaluation of the Green's function

$$
G_{A B}^{\mathcal{B}}\left(x-x^{\prime}\right)=\frac{i}{2} \epsilon_{i j n} \theta\left(t-t^{\prime}\right)\left\langle\left[J_{A}^{i}(x), J_{B}^{j}\left(x^{\prime}\right)\right]\right\rangle
$$

that reduces to

$$
G_{A B}^{\mathcal{B}}=\frac{1}{2} \sum_{f, g} T_{A}^{g} T_{B}^{g} f \frac{1}{\beta} \sum_{\tilde{\omega} f} \int \frac{d^{3} q}{(2 \pi)^{3}} \epsilon_{i j n} \operatorname{Tr}\left[S_{f}^{f}(q) \gamma^{i} S_{f}^{f}(q+k) \gamma^{j}\right],
$$

being $S(q)$ the fermion propagator and $\beta$ the inverse temperature. This is the diagram of Figure 3.1 after exchanging the external energy-momentum tensor by a current $J_{B}^{j}$. The final result reads 67

$$
\sigma_{A B}^{\mathcal{B}}=\frac{1}{4 \pi^{2}} \sum_{f, g=1}^{N} T_{A f}^{g} T_{B f}^{g} \mu^{f}=\frac{1}{8 \pi^{2}} \sum_{C} \operatorname{Tr}\left(T_{A}\left\{T_{B}, H_{C}\right\}\right) \mu_{C} .
$$

For the Chiral Vortical Conductivity the calculation is similar [70]. The interesting correlator is

$$
G_{A}^{\mathcal{V}}\left(x-x^{\prime}\right)=\frac{i}{2} \epsilon_{i j n} \theta\left(t-t^{\prime}\right)\left\langle\left[J_{A}^{i}(x), T^{0 j}\left(x^{\prime}\right)\right]\right\rangle,
$$

see Figure 3.1. The final outcome turns out to be [70]

$$
\begin{aligned}
\sigma_{A}^{\mathcal{V}} & =\frac{1}{8} \sum_{f=1}^{N} T_{A f}^{f}\left[\left(\mu^{f}\right)^{2}+\frac{\pi^{2}}{3} T^{2}\right] \\
& =\frac{1}{16 \pi^{2}}\left[\sum_{B, C} \operatorname{Tr}\left(T_{A}\left\{H_{B}, H_{C}\right\}\right) \mu_{B} \mu_{C}+\frac{2 \pi^{2}}{3} T^{2} \operatorname{Tr}\left(T_{A}\right)\right] .
\end{aligned}
$$

The contribution proportional to $\mu^{2}$ was firstly observed by a computation in holography [87, 88. The term $\sim T^{2}$ is more interesting. It was observed in 70 firstly for free fermions by the direct computation sketched here. The fact that it is proportional to $\operatorname{Tr}\left(T_{A}\right)$ strongly suggests that it can be related to the Mixed Gauge-Gravitational anomaly. By means of QFT it is difficult to demonstrate it due to the fact that we do not have control over the anomaly contributions. However, in holography we can tune the anomaly parameter at will and, as will be shown in Chapter 5 , the term proportional to $T^{2}$ has its origin in the presence of a Mixed anomaly.

\section{Vector/Axial splitting}

To finish this section, let us particularize to the case of one vector and one axial currents with chemical potential $\mu_{R}=\mu+\mu_{5}$ and $\mu_{L}=\mu-\mu_{5}$. The charges are $q_{\{V, A\}}^{R}=(1,1)$ and 


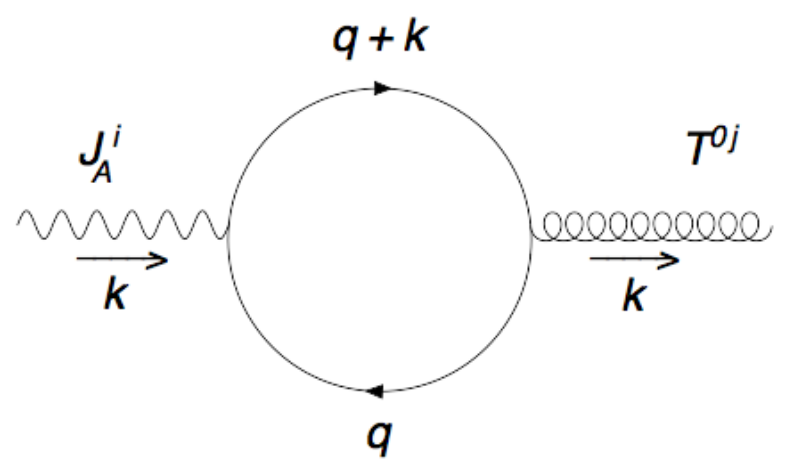

Figure 3.1: The 1-loop diagram that contributes at leading order to the CVC.

$q_{\{V, A\}}^{L}=(1,-1)$ for one right-handed and one left-handed fermion. Assuming a vector magnetic field, we get

$$
\begin{array}{rlrl}
\sigma_{V V}^{\mathcal{B}} \equiv \sigma_{C M E} & =\frac{\mu_{5}}{2 \pi^{2}} ; & \sigma_{A V}^{\mathcal{B}} \equiv \sigma_{C S E}=\frac{\mu}{2 \pi^{2}}, \\
\sigma_{V}^{\mathcal{V}}=\frac{\mu \mu_{5}}{2 \pi^{2}} ; & \sigma_{A}^{\mathcal{V}}=\frac{\mu_{5}^{2}+\mu^{2}}{4 \pi^{2}}+\frac{T^{2}}{12} .
\end{array}
$$

For the the vector Vortical Conductivity $\sigma_{V}^{\mathcal{V}}$ the contributions coming from the gravitational anomaly cancel between right- and left-handed fermions.

The chiral conductivities can be computed in dimensions different from four, as long as the corresponding anomalies can be accommodated. We refer the interested reader to 89 92] for different approaches to that question. 


\section{Chapter 4}

\section{Relativistic Superfluids}

Superfluidity is a very well-known phenomenon, that can be described as the capability of a fluid of flowing without friction through thin capillaries 1 . However, a superfluid is more than just that. For instance, one can observe that several vortices generate when we induce some rotation in he fluid; moreover, the speed at which temperature is transported in a superfluid is much larger than in ordinary fluids.

The paradigmatic example of a (non-relativistic) superfluid is liquid Helium. We know that, below certain temperature $\sim 2.7 \mathrm{~K}$, the liquid acquires the exotic properties described above. In modern language, we say that a phase transition has taken place, and a symmetry has gotten spontaneously broken (a $U(1)$ symmetry in the case of superfluid Helium).

Breaking a symmetry spontaneously does not mean that the charge is lost. Only that some of that charge gets stored in a single vacuum quantum state called "condensate".

At this point it is important to emphasize the difference between the existence of a BoseEinstein condensation (BEC), that represents the existence of a well-populated condensate, and the presence of superfluidity. Superfluidity is a property of the excited states, concretely, it is feasible if the system finds energetically favourable to create quasiparticles. For instance, in superfluid Helium the spectrum of excitations are superfluid phonons and rotons, the latter being gapped. There is a famous (non-relativistic) result by Landau [93] that establishes that, at zero temperature, there is a limiting superfluid velocity before dissipation is produced

$$
V_{c}=\min \frac{E(k)}{k},
$$

where $E(k)$ is the energy of the excitations as a function of momentum (dispersion relation). If $V>V_{c}$, the system starts creating rotons (normal component of the fluid) and dissipation occurs. In order to get 4.0.1, we impose conservation of the energy and momentum. If the fluid (of mass $M$ ) wants to slow down from $v$ to $v^{\prime}<v$ by creating an excitation we have

$$
\begin{aligned}
\frac{M v^{2}}{2} & =\frac{M v^{\prime 2}}{2}+E(k), \\
M \vec{v} & =M \vec{v}^{\prime}+k .
\end{aligned}
$$

Eliminating $v^{\prime}$ we arrive at $\vec{v} \cdot \vec{k}-\frac{k^{2}}{2 M}=E(k)$. Even in the best scenario, in which $M>>1$ and $\vec{v}$ and $\vec{k}$ are parallel, we get a limiting velocity given by 4.0.1).

\footnotetext{
${ }^{1}$ Thin capillaries are necessary to avoid the generation of vortices, which work as regions where there is no superfluid. The bulk velocity is in this way damped.
} 
Notice that the Landau criterion also tells us that below $V_{c}$ the superfluid would not be capable of slowing down, for there are no excitations to generate. Therefore, the system moves without dissipation. The main difference between a superfluid and an ordinary fluid is that the latter features $V_{c}=0$ (for instance, vortices in ordinary fluids can be generated at the cost of a very small amount of energy).

However, it is not true in general that supefluidity is present whenever a condensate is generated. The most trivial example is the gas of non-interacting bosons, for which a phase transition takes place in the infinite volume limit. However, there exist more sophisticated systems that feature a BEC but are not superfluids. We will provide with a holographic realization of one of those systems in Chapter III.

\subsection{A model for a superfluid}

To understand where the exotic properties of a superfluid come from, the best way to proceed is to work with a toy model that admits a BEC. This is achieved by considering a $\lambda|\varphi|^{4}$ theory $\left.\right|^{2}$

$$
\mathcal{L}=\partial_{\mu} \varphi \partial^{\mu} \varphi^{*}-m^{2}|\varphi|^{2}-\lambda|\varphi|^{4}
$$

where $\varphi$ is a complex scalar field that could be associated to some possibly composite scalar operator, $m$ is the mass and $\lambda>0$ a coupling constant. The above Lagrangian is invariant under a global $\mathrm{U}(1)$ symmetry $\varphi \rightarrow e^{i \alpha} \varphi$. Parametrizing the complex scalar field as $\varphi(x)=\frac{e^{i \psi(x)}}{\sqrt{2}} \rho(x)$ we get equations of motion for $\psi$ and $\rho$ that admit a simple but non-trivial solution which preserve translation invariance

$$
\psi=p_{\mu} x^{\mu} ; \quad \rho=\sqrt{\frac{-p^{2}-m^{2}}{\lambda}} .
$$

where $p_{\mu}$ is arbitrary, i.e. fully determined by the boundary conditions (notice that $p^{2}<0$ ). If $-p^{2}>m^{2}$, the background scalar field acquires a non-sourced VEV and the choice of a particular $p_{\mu}$ makes (4.1.4) break the global U(1) symmetry. The BEC is related to such a non-vanishing VEV; one measure of the population of the fundamental state (the so-called superfluid density) is $\rho^{2}$. The field $\psi(x)$ is usually called the Goldstone field and it will be of fundamental importance from now on. In the broken phase, the source $\partial_{\mu} \psi \equiv-\tilde{\xi}_{\mu}$ is invariant under the $\mathrm{U}(1)$ symmetry and hence one can study the response of the system under its influence. Solution (4.1.4) suggests that there exists an equilibrium state in which $\tilde{\xi}_{\mu} \neq 0$, meaning that the new type of source will affect even the thermodynamic properties of the system. This is indeed what occurs; the ideal hydrodynamic constitutive relations (at zero temperature) read ${ }^{3}$

$$
\begin{aligned}
j^{\mu} & =n_{s} v^{\mu}, \\
T^{\mu \nu} & =\epsilon_{s} v^{\mu} v^{\nu}+P^{\mu \nu} P_{s} .
\end{aligned}
$$

The time-like vector $v^{\mu}=\frac{\tilde{\xi}^{\mu}}{\sqrt{-\tilde{\xi}^{2}}}$ is the so-called normalized superfluid velocity, i.e. $v^{\mu} v_{\mu}=$ -1 and $P^{\mu \nu}$ the corresponding projection operator. $P_{s}$ is the pressure at zero temperature,

\footnotetext{
${ }^{2}$ For an effective-theory approach to superfluids, see 94,96 .

${ }^{3}$ Strictly speaking, one is forced to consider also the hydrodynamics of $\psi$ due to the fact that it is a massless field. We will not take this issue into account here because it is not of fundamental importance for our purposes.
} 
$n_{s}$ the charge density and $\epsilon_{s}$ the energy. The superfluid rest frame $v^{\mu}=(1, \overrightarrow{0})$ diagonalizes $T^{\mu \nu}$ and makes $j^{\mu}=n_{s} \delta_{t}^{\mu}$. From here one gets

$$
\begin{aligned}
\epsilon_{s}+P_{s} & =n_{s} \mu_{s}, \\
d P_{s} & =n_{s} d \mu_{s}+\frac{1}{2} f d \tilde{\xi}^{2},
\end{aligned}
$$

where $f$ is a function conjugate to $\tilde{\xi}^{2}$ and $\mu_{s} \equiv-\tilde{\xi}_{t}$. Furthermore, the quantity $v_{s}^{\mu}=P^{\mu \nu} \tilde{\xi}_{\nu}$ is identified with the superfluid velocity $\mathbf{v}_{s}$. Moreover one can show that $n_{s}=\sqrt{-\tilde{\xi}^{2}} \rho^{2}$ and therefore $n_{s}$, which behaves as a superfluid charge density, is tightly connected to $\rho^{2}$, as aforementioned.

Now, let us gauge 4.1.3 weakly. This implies considering a non-dynamical gauge field coupled to $\varphi$, namely $\partial_{\mu} \varphi \rightarrow \partial_{\mu} \varphi-A_{\mu} \equiv D_{\mu} \varphi$ in 4.1.3 . Expanding in terms of $\psi$ and $\rho$, one can check that the resulting Lagrangian features a gauge symmetry

$$
\psi(x) \rightarrow \psi(x)+\alpha(x) ; \quad A_{\mu} \rightarrow A_{\mu}+\partial_{\mu} \alpha(x) .
$$

Along with the usual electric and magnetic fields, the source $\xi_{\mu} \equiv \partial_{\mu} \psi-A_{\mu}$ is also gaugeinvariant in the broken phase. At the level of ideal hydrodynamics, one can imagine constitutive relations similar to $4.1 .5-4.1 .6$, in which $v^{\mu}$ is now defined as $v^{\mu}=\frac{\xi^{\mu}}{\sqrt{-\xi^{2}}}$. Taking the analogue of (4.1.5) and using (4.1.8) to fix a gauge in which $\psi=0$, one gets

$$
j^{\mu}=-\frac{n_{s}}{\mu_{s}} A^{\mu} .
$$

The above relation is known as the London equation and it is at the core of the Meissner effect. Making use of Maxwell equations along with 4.1.9 one is led to the equation $\nabla^{2} \vec{B}=\frac{1}{\lambda^{2}} \vec{B} ; \quad \lambda^{2}=\sqrt{\frac{\mu_{s}}{4 \pi n_{s}}}$ for a magnetic field $\vec{B}$ aplied on a sample of superconducting material. Solving it we find

$$
B_{z}(x)=B_{0} e^{-x / \lambda},
$$

which can be interpreted as if the external gauge field became effectively massive $M \sim 1 / \lambda$ inside the sample. The Meissner effect has been observed in holography in [3, 97. Another consequence of equation 4.1 .9 is that it can be written as $j^{\mu}=-\frac{i n_{s}}{\mu_{s} \omega} E^{\mu}$, being $E^{\mu}$ the electric field. Therefore, the DC conductivity becomes infinite. We will observe that phenomenon in Section 8.2. It is worth mentioning also that after using (4.1.8) to gauge away the Goldstone field, we can introduce the superfluid chemical potential $\mu_{s}$ and supervelocity $\mathbf{v}_{s}$ as background values of $A_{0}$ and $A_{i}$ respectively. This could also have been anticipated by the form of $(4.1 .9)$, where it is clear that the temporal and spatial components of the gauge field act as sources for the superfluid charge density and the supervelocity. This trick will be used frequently in the holographic approach of Chapter 8

So far our discussion has been limited to zero temperature. At finite temperature the description is more involved because generically there are quasiparticles (normal component of the fluid) coexisting with the BEC. A hydrodynamic formulation similar to 4.1.5 - 4.1.6) is still possible, but at finite temperature it is necessary to take into account also the velocity $u^{\mu}$ associated to the normal component and its contribution to the

\footnotetext{
${ }^{4}$ Should we include a $F^{2}$ term for $A_{\mu}$, the Higgs mechanism will take place. Here we are not interested in that situation.
} 
thermodynamic relations 98 100]. Notice that for infinitely large systems the Landau criterion is better formulated at finite temperature for one can consider the reference frame which is at rest with respect to the normal component (i.e. $\left.u^{\mu}=(1,0,0,0)\right)$ and refer the superfluid velocity to that reference fram 5 . As shown in [100], for the theory $[4.1 .3$ at $m=0$ and perturbatively small temperature there appear inconsistencies if the supefluid velocity satisfies

$$
\mathbf{v}_{s}^{2}=1 / 3
$$

One could ask whether those issues arise as manifestations of the Landau Criterion. If this was so, the we would be led to conclude that $V_{c}(T \simeq 0)=1 / \sqrt{3}$. In the following we show explicitly that $1 / \sqrt{3}$ is indeed the velocity of sound of excitations whose dispersion relation is $\omega(k)=v_{s} k$. This makes clear that the divergencies observed are the effect of the Landau Criterion at work.

\subsection{Spectrum of excitations: Sound}

In the unbroken phase, the spectrum of excitations contains a massless mode known as first sound and associated to density waves in ordinary fluids (the usual "sound" with $\omega^{2} \simeq c_{s}^{2} k^{2}$ and $\left.c_{s}^{2}=\left.\frac{\partial p}{\partial \rho}\right|_{S, N}\right)$. However, in the broken phase the interplay between the superfluid and the ordinary fluid generates in addition a new mode called second sound. The excitation of the first sound provokes an in-phase disturbances of the normal and superfluid components, such that their contribution to the variation of the total density adds up. On the other hand, the second sound represents the wave-like propagation of heat. In this case the components move almost in opposite phase and the total densities remain unaltered. We therefore conclude that in a superfluid temperature is transferred as a wave (fastly), contrary to the case of ordinary fluids, in which conserved quantities (such as heat) satisfy a diffusion equation and hence they are transferred very slowly. The physical consequences of the existence of a second sound, for example, is that the superfluid reaches thermal equilibrium rapidly after a gradient of temperature is applied in one small region. Moreover, the famous fact that superfluid Helium does not boil is a consequence of the fast propagation of heat: all the evaporation occurs at the surface.

A perturbation of the system will generically excite both modes. There exists the possibility that only the superfluid component flows due to the perturbations. This can be achieved by including fine scatterers that prevent the normal component to flow, i.e. $\delta u^{i}=0$ 101, 102. In such a situation the momentum of the fluid will be generically not conserved, due to the fact that we are forcing the scatterers to be infinitely massive. As shown for instance in 102, the resulting perturbation is a combination of first and second sound called fourth-sound, which presents a dispersion relation of the form $w^{2}=v_{4}^{2} k^{2}$ with

$$
v_{4}^{2}=\frac{\rho}{\mu}\left(\frac{\partial \mu}{\partial \rho}\right)_{s}
$$

where $\rho, \mu, s$ are the charge density, chemical potential and entropy density in equilibrium, respectively. It can be shown that the above equation is the phase velocity of the second sound close to $T_{c}$. At low temperatures, conformal fluids must obey $\rho \propto \mu^{d-1}$ (there is no

\footnotetext{
${ }^{5}$ At zero temperature there are not quasiparticles present and therefore all the reference frames are supposed to be equivalent. However, the Landau criterion...
} 
other scale in the system) and hence $\lim _{T \rightarrow 0} v_{4}^{2}=\frac{1}{d-1}$. This turns out to be the velocity of first sound close to $T=0$. So we conclude that the fourth sound interpolates between first sound, at low temperatures, and second sound close to $T_{c}$. We will observe fourth sound in holographic models restricted to the probe approximation, to be defined later on. Encountering the fourth sound at zero temperature is relatively easy. Let us consider an euclidean version of 4.1 .3 at finite chemical potential

$$
\mathcal{L}=\left(\partial_{0}+\mu\right) \varphi^{*}\left(\partial_{0}-\mu\right) \varphi+\partial_{i} \varphi^{*} \partial_{i} \varphi+m^{2} \varphi^{*} \varphi+\lambda\left(\varphi^{*} \varphi\right)^{2} .
$$

We observe that at $\mu_{c}=M$ a phase transition takes place and the $\mathrm{U}(1)$ symmetry gets spontaneously broken. We choose the $\operatorname{VEV}$ for $\varphi$ to $\operatorname{read} \varphi_{0}=\frac{1}{\sqrt{2}} \rho ; \rho^{2}=\frac{\mu^{2}-M^{2}}{\lambda}$. Now we perturb the background as $\varphi(x)=\frac{1}{\sqrt{2}} \rho+\varphi_{1}(x)+i \varphi_{2}(x)$ to get a sencond order Lagrangian that reads

$$
\mathcal{L}^{(2)}=\sum_{i=1}^{2} \partial_{\mu} \varphi_{i} \partial_{\mu} \varphi_{i}+2 i \mu\left(\varphi_{1} \partial_{0} \varphi_{2}-(1 \leftrightarrow 2)\right)-\lambda \phi^{2} \varphi_{2}^{2} .
$$

Diagonalizing the mass matrix, we find a massless mode with a dispersion relation

$$
\omega^{2}(k)=\frac{\mu^{2}-m^{2}}{3 \mu^{2}-m^{2}} k^{2}+\mathcal{O}\left(k^{4}\right) .
$$

In the hydrodynamic limit, the dispersion relation is hence linear with the momentum $k$ with a velocity of sound $v_{s}=\sqrt{\frac{\mu^{2}-m^{2}}{3 \mu^{2}-m^{2}}}$. The conformal limit of the theory under consideration is obtained when $m=0$. In that case

$$
v_{s}(m=0)=\frac{1}{\sqrt{3}} .
$$

This value is precisely the expected one for a four dimensional conformal field theory at zero temperature. Furthermore, it is compatible with a critical velocity $V_{c}=\frac{1}{\sqrt{3}}$ (see the discussion at the end of Section 4.2 .

To summarize, we have presented the picture of a weakly coupled superfluid, resulting from the breaking of a global U(1) symmetry, and its spectrum of excitations. At zero temperature there exists a massless Goldstone mode ${ }^{6}$ with dispersion relation $\omega^{2}=v_{s}^{2} k^{2}$ and $v_{s}$ given by (4.2.16) in the conformal limit. Moreover, we have pointed out that the Landau criterion of superfluidity for this system is satisfied, with a resulting critical velocity $V_{c}(m=0, T \simeq 0)=\frac{1}{\sqrt{3}}$. Even though the final picture is perfectly consistent, one could ask for generalizations of it. For instance, what would happen at strong coupling? Could the massless modes present dispersion relations different from $\omega \sim k$ ? To what extent are our conclusions model dependent? We address some of these questions below.

\subsection{Generalizations}

At this point let us elaborate on some directions that can be followed to extend the discussion.

\footnotetext{
${ }^{6}$ It is also often called Nambu-Goldstone boson. For simplicity we will refer to it as Goldstone boson or Goldstone mode throughout the text.
} 


\section{Model dependence}

One could argue against the convenience of using a simple model such as $\lambda|\varphi|^{4}$. Considering suitable generalizations of (4.1.3), it will result in drastic implications on the conclusions that have been reached before. In the following we present an argument in support of the generality of 4.1 .3 in the hydrodynamic limit. One can write down an effective theory for the Goldstone field by simply substituting $\varphi(x)=\frac{e^{i \psi(x)}}{\sqrt{2}} \rho(x)$ into the Lagrangian, to obtain

$$
\mathcal{L}_{e f f}=\frac{\rho^{2}}{2}\left(\partial_{\mu} \psi \partial^{\mu} \psi-m^{2}\right)-\frac{\lambda}{4} \rho^{4}
$$

with $\rho$ (and only $\rho$ ) given by 4.1.4 7 . As aforementioned, 4.3.17) is invariant under global shifts of the Goldstone $\psi \rightarrow \psi+\alpha$.

We can now ask ourselves about possible corrections to 4.3.17) compatible with the shift symmetry. The conclusion is that any suitable term $\sim \mathcal{O}\left(\psi^{n}\right)$ must be accompanied by at least $n$ derivatives. In other words, the building block of a generic theory is $\partial \psi$. An arbitrary interacting theory would thus contain more than two derivatives and would in turn be suppressed in the hydrodynamic limit. Hence, we conclude that 4.1.3 provides with the leading contribution at low energies: 8 .

\section{Strong coupling limit}

The above argument also ensures that interactions among the Goldstone particles will be suppressed at low energies. What is therefore a strongly coupled superfluid? As in the case of ordinary fluids, the weakly coupled sector of hydrodynamic modes will interact with a (strongly coupled) thermalized sector. We expect this to have important implications at finite temperature, for the presence of the strongly coupled sector influences the physics of dissipation, that is, the imaginary part of the frequency of the physical modes. It is therefore plausible that the Goldstone particles cannot be anymore understood as quasiparticles (as it is always the case at weak coupling in four dimensions). All these issues will be studied by means of holography in Chapter 8 .

\section{Other types of condensates}

So far we have restricted ourselves to condensation of scalar operators. In principle, however, it is also possible that the condensate behaves as a vector ( $p$-wave superfluids) or even a rank-2 tensor (d-wave superfluids). The spontaneous breaking of internal symmetries is in those cases accompanied by the loss of parity and rotational invariance. We refer the reader to 103 104 for field theoretical approaches to p-wave superfluids. We will elaborate briefly on these less common superfluids in Chapter 10, focusing mostly on the strong coupling regime. Moreover, we will construct a phase in which both s- and p-wave condensates coexist.

\footnotetext{
${ }^{7}$ The reason why we consider $\rho$ to be on-shell is that it is a massive field and we can neglect its dynamics as long as we are concerned with low-energy processes.

${ }^{8}$ There is a caveat to this argument: it is plausible to consider a Wess-Zumino term that change as a total derivative under a shift of $\psi$ and contains fewer derivatives than 4.3.17. We will not take into account such terms here.
} 


\section{Dispersion relations other than $\omega \sim k$}

One could also ask what would occur if we forbid terms of the form $\partial_{\mu} \psi \partial^{\mu} \psi 9^{9}$ and hence we force the effective Lagrangian to start at higher order in derivatives. Reaching somehow a non-relativistic limit, such theories would be expected to present massless modes with dispersion relations starting at higher order in momentum (for example $\omega^{2} \sim k^{4}+\ldots$ ). This is a natural question that will not be adressed here (see for instance 106] ${ }^{10}$, Despite of the above, it is not obvious a priori that the only possible dispersion relations must be of the form $\omega \sim k$ in the hydrodynamic limit. To bring light onto this question, we present below some general considerations of the low energy spectrum of theories with BECs. We will see that the collection of available dispersion relations is not restricted to $\omega \sim k$ as long as we force some kind of breaking of Lorentz invariance. The existence of massless modes in the spectrum of superfluids and the form of their dispersion relations are very robust and depend only on the structure of the broken symmetries.

\subsection{Goldstone Theorem and type II NG bosons}

In the context of condensed matter physics it has been pointed out long ago in 107 that certain multicomponent superfluids present unusual Goldstone modes with quadratic dispersion relation. In the high energy context such models have been considered as models for Kaon condensation in the color-flavor locked phase of QCD in [108,109 again emphasizing the existence of the quadratic Goldstone mode. It seems useful to collect now some of the known theorems on Goldstone bosons (a very useful review on symmetry breaking and Goldstone modes is [110]). First we have of course the actual Goldstone theorem. Its proof assumes the existence of a conserved current $j^{\mu}$ such that the broken charge is $Q=\int d^{d} x j^{0}$ (with $d$ spatial dimensions). The theorem then states that spontaneous breaking of a continuous global symmetry implies the existence of a mode whose energy fulfills

$$
\lim _{k \rightarrow 0} \omega(k)=0 .
$$

The theorem by itself does not make any statement about the number of these modes, nor does it fix the $k$-dependence of the frequency. In the presence of Poincaré symmetry one can make however a stronger statement, namely that the dispersion relation of the Goldstone mode has to be linear and that the number of Goldstone bosons equals the number of broken generators.

Lorentz symmetry might be absent however, either in principle such as in non-relativistic field theories or the system under consideration might be in a Lorentz symmetry breaking state, such as being at finite temperature or density. In these cases another theorem classifies Goldstone bosons as type I if their energy vanishes as an odd power of the momentum or as type II if their energy vanishes as an even power of the momentum in the zero momentum limit. The number of type I and type II Goldstone bosons has to fulfill then

$$
n_{I}+2 n_{I I} \geq N_{B G},
$$

where $N_{B G}$ is the number of broken generators 111 . The number of type I and type II Goldstone bosons can be further constrained. Upon assuming that the broken symmetry

\footnotetext{
${ }^{9}$ For instance, by demanding that $\psi$ corresponds to a galileon 105 .

${ }^{10}$ We do not discard however that the type II NG boson, to be defined in Section 4.4 below, could arise from an effective theory with an enhanced shift symmetry, at least at lowest order in derivatives.
} 
generators obey $\left\langle\left[Q_{a}, Q_{b}\right]\right\rangle=B_{a b}$ the number of Goldstone bosons has to fulfill 112 114 (see also [110,115, 116] for more on counting rules of Goldstone bosons).

$$
n_{I}+n_{I I}=N_{B G}-\frac{1}{2} \operatorname{rank}(B) .
$$

\subsubsection{A field theoretical model with type II Goldstone boson}

Motivated by the physics of Kaon condensation in the color-flavor locked phase of QCD the authors of 108, 109 studied QCD at a nonzero chemical potential for strangeness. It was shown that at a critical value of the chemical potential equal to the Kaon mass, Kaon condensation occurs through a continuous phase transition. Moreover, a Goldstone boson with the non-relativistic dispersion relation $\omega \sim k^{2}$ appears in the Kaon condensed phase. To illustrate this fact, they considered the following (Euclidean) toy model:

$$
\mathcal{L}=\left(\partial_{0}+\mu\right) \phi^{\dagger}\left(\partial_{0}-\mu\right) \phi+\partial_{i} \phi^{\dagger} \partial_{i} \phi+M^{2} \phi^{\dagger} \phi+\lambda\left(\phi^{\dagger} \phi\right)^{2},
$$

where $\phi$ is a complex scalar doublet,

$$
\phi=\left(\begin{array}{l}
\phi_{1} \\
\phi_{2}
\end{array}\right) .
$$

As long as $\mu<M$ the masses of the four excitations in the model are the positive roots in $\omega$ of

$$
(\omega \pm \mu)^{2}=M^{2}
$$

All are doubly degenerate. It is straightforward to check that at $\mu=M$ the global $U(2)$ symmetry gets broken and the new vacuum can be chosen to be

$$
\phi=\frac{1}{\sqrt{2}}\left(\begin{array}{l}
0 \\
v
\end{array}\right), \quad \text { with } v^{2}=\frac{\mu^{2}-M^{2}}{\lambda} .
$$

Studying the fluctuations of the doublet $\phi$ around this background one finds two massless and two massive modes with the following dispersion relations:

$$
\begin{aligned}
\omega_{1}^{2} & =\frac{\mu^{2}-M^{2}}{3 \mu^{2}-M^{2}} k^{2}+\mathcal{O}\left(p^{4}\right), \\
\omega_{2}^{2} & =6 \mu^{2}-2 M^{2}+\mathcal{O}\left(k^{2}\right), \\
\omega_{3}^{2} & =k^{2}-2 \mu \omega_{3}, \\
\omega_{4}^{2} & =k^{2}+2 \mu \omega_{4} .
\end{aligned}
$$

If we concentrate on the positive roots we see that $\omega_{1}$ is a normal, linear Goldstone mode. In the conformal limit, which in this theory corresponds just to $M=0$, we recover the expected dependence $\omega_{1}^{2}=\frac{1}{3} k^{2}$ for the fourth sound in four dimensions (see the discussion under equation (4.2.12)). Moreover, right at the phase transition $\mu=M$ and hence $\omega_{1}^{2} \sim \mathcal{O}\left(k^{4}\right)$, so at that point in phase space even the type I NG boson has a quadratic dispersion relation.

The positive root of equation 4.4.27 is

$$
\omega_{3}=\frac{k^{2}}{2 \mu}+O\left(k^{4}\right)
$$


This is the type II Goldstone mode. It has formally a non-relativistic dispersion relation. Since the underlying theory has however Lorentz invariance there is of course also a negative energy mode with quadratic dispersion. This arises as the negative root of $\omega_{4}$. Finally $\omega_{2}$ and $\omega_{4}$ are gapped modes with

$$
\omega_{4}=2 \mu+O\left(k^{2}\right) .
$$

Since the symmetry breaking pattern is $U(2) \rightarrow U(1)$ there are three broken generators but only two massless Goldstone modes in the spectrum. This model fulfills all the counting theorems noted in the introduction. In particular the Chadha-Nielsen rule (4.4.19) is exactly saturated. The role of $\omega_{4}$ is special. It is the mode that pairs up with the type II Goldstone mode in the dispersion relations (4.4.27) and 4.4.28). It has been argued that this mode is a universal feature and that its energy at zero momentum is exact and protected against quantum corrections 115, 117, 118. The spectrum obtained from this model is summed up in Figure 4.1. In our holographic models we will look for this special gapped partner mode of the type II Goldstone mode. This simple Lagrangian model

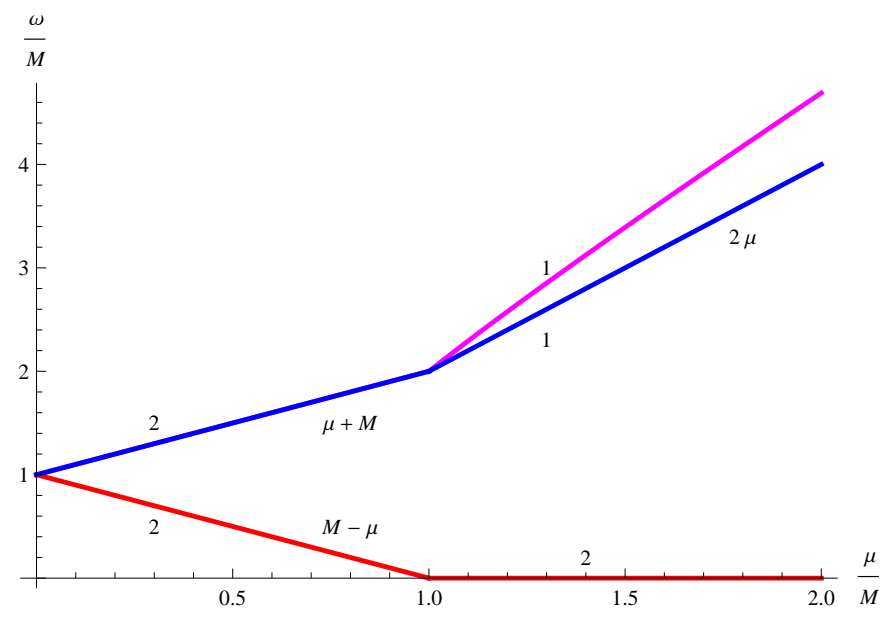

Figure 4.1: The spectrum of the field theoretical model. Below the critical value $\mu=M$ there are four massive modes. The masses are $M-\mu$ and $M+\mu$, the numbers indicate that they are doubly degenerate. In the broken phase $\mu>M$ there are two Goldstone modes with exactly zero mass and two gapped modes. The special gapped mode has mass $2 \mu$.

serves as our motivation and guideline to construct a holographic model featuring type II Goldstone modes. In fact we can use the same kind of matter Lagrangian in a holographic setup.

\subsection{Superfluid (anomalous) hydrodynamics}

Equations 4.1.5-4.1.6 represent the constitutive relations of an ideal superfluid at zero temperature. After weakly gauging, we saw that one can define a gauge-invariant source in the broken phase of the form

$$
\xi_{\mu}=\partial_{\mu} \psi-A_{\mu}
$$

When constructing the hydrodynamic constitutive relations for a non-ideal superfluid at finite temperature, followng the procedure of Section 3.2, one has to consider 4.5.31 (and 
its derivatives) in addition to the contributions already present for ordinary fluids. This makes the analysis much more involved [96].

Here we briefly discuss the parity-odd sector of the resulting effective theory to give some insights into the general framework in which Part 12 of this work is embedded. Let us consider that metric perturbations are set to zero throughout the following discussion, so that only generic components of gauge fields are turned on. In addition to those external sources, the effective theory contains a dynamical Goldstone field (as 4.3 .17 does) ${ }^{11}$. Thus, appart from electric and magnetic fields, to first order in derivatives there is also space for

$$
S_{1} \equiv \epsilon^{i j k} \zeta_{i}^{e q \cdot} \partial_{j} \zeta_{k}^{e q \cdot} ; \quad V_{2}^{i} \equiv \epsilon^{i j k} \zeta_{j}^{e q \cdot} \partial_{k} A_{0} ; \quad V_{5}^{i} \equiv \frac{1}{T^{2}} \epsilon^{i j k} \zeta_{j}^{e q \cdot} \partial_{k} \zeta_{e q .}^{2},
$$

where $\zeta^{\nu}=P^{\nu \mu} \xi_{\mu}$ (the projector $P^{\nu \mu}$ has been defined in Section 2.2). The label "eq." means that $\psi$ has to be evaluated on-shell, i.e. as a functional of the sources $A_{0}$ and $A_{i}$. Recall that we are focusing only on the source terms affecting the anomalous part of the current. It turns out that the zeroth-component of the the partity-odd sector of the covariant current reads

$$
J_{0}^{\text {odd }}=g_{1, \hat{\nu}} S_{1},
$$

where $g_{1, \hat{\nu}}$ is a function that depends on $T, \mu$ and the background supervelocity $\left|\mathbf{v}_{s}\right|^{2} \equiv \zeta_{0}^{2}$. The spatial component $J_{i}^{\text {odd }}$ feature more terms and will not be included here (see 96$]$ for details).

The new terms discussed above lead to interesting consequences for the anomalous transport of Chiral Superfluids. For example, the CMC will not be fully determined by anomalies anymore. Moreover, new types of anomalous transport arise (under the name of Chiral Electric Effect and Chiral Charge Generation Effect). Part 12 below is devoted to the analysis of the new features making use of a holographic setup.

\footnotetext{
${ }^{11}$ The effective theory must contain at least the Goldstone mode. The theory resulting from integrating out a dynamical massless mode is non-local at any arbitrary small scale and therefore one has to keep the Goldstone field unaltered.
} 


\section{Part II}

\section{Anomalous Transport in Holography}




\section{Chapter 5}

\section{Holographic Mixed \\ Gauge-Gravitational anomaly and transport}

As pointed out in Section 3 , right after the discovery of the CVC via the Fluid/Gravity correspondence, an study via Kubo formulae was made, obtaining the same results. However, a QFT computation of the CVC for fermions at zero coupling revealed a new term proportional to the temperature squared in $\sigma_{A}^{\mathcal{V}}$, equation 3.0 .5 .

Contrary to the case in QFT, in usual holographic bottom-up models the implementation of the anomaly allows us to turn off the anomaly coefficient at will. This has the advantage that we can prove the conjecture made in [70], namely that the new term appearing in the CVC is related to the Mixed Gauge-Gravitational anomaly, using holography. The new term that we have to include in the action in order to implement such an anomalous ${ }^{1}$ contribution is

$$
\delta S=\frac{\lambda}{16 \pi G} \int d^{5} x \sqrt{-g} \epsilon^{M N P Q R} A_{M} R_{B N P}^{A} R^{B}{ }_{A Q R}
$$

Naively one could expect that the above only contributes to terms with at least three transverse derivatives, due to the definition of the Riemann tensor. However, we will show below that it is not sd?

Moreover, the computation at strong coupling served to show that the anomalous transport coefficients do not feel the strength of the coupling, which at that time was interpreted as an indication of the existence of a non-renormalization theorem for chiral conductivities. We know now that the lack of dependence on the coupling is only true in the absence of gauge fields that unavoidably contribute to the anomaly trough quantum corrections $121-123$. We cannot see such effects using the holographic methods used commonly because all the bulk gauge fields correspond to non-dynamical sources at the boundary.

Moreover, usual methods invoking the positivity of the entropy current do not fix completely the chiral conductivities. There are several coefficients $\beta_{a}$ and $\gamma$ that arise as integration constants, as elaborated on in Section 3.2. The constant $\gamma$ is forced to vanish due to CPT invariance, but $\beta_{a}$ is in principle free. We will see, however, that $\beta_{a}$ is actually fixed by the Mixed Anomaly.

\footnotetext{
120 .

${ }^{1}$ For a purely graviational analysis of similar Chern-Simons corrections to General Relativity, see 119

${ }^{2}$ Undestanding why the Mixed anomaly contributes at first order in derivatives is not trivial 81,121
} 
The work presented below served to obtain such a temperature-dependent term also at strong coupling, establishing that it is the same at zero coupling and also confirming that such a term in the CVC coefficient is completely determined by the Mixed GaugeGravitational anomaly.

\subsection{Holographic Model}

In this section we will define our model. We start by fixing our conventions. We choose the five dimensional metric to be of signature $(-,+,+,+,+)$. The epsilon tensor has to be distinguished from the epsilon symbol. The symbol is defined by $\epsilon(r t x y z)=+1$ whereas the tensor is defined by $\epsilon_{A B C D E}=\sqrt{-g} \epsilon(A B C D E)$. Five dimensional indices are denoted with upper case latin letters. We define an outward pointing normal vector $n_{A} \propto g^{A B} \frac{\partial r}{\partial x^{B}}$ to the holographic boundary of an asymptotically AdS space with unit norm $n_{A} n^{A}=1$ so that the induced metric takes the form

$$
h_{A B}=g_{A B}-n_{A} n_{B} .
$$

In general a foliation with timelike surfaces defined through $r(x)=$ const can be written as

$$
d s^{2}=\left(N^{2}+N_{A} N^{A}\right) d r^{2}+2 N_{A} d x^{A} d r+h_{A B} d x^{A} d x^{B} .
$$

The Christoffel symbols, Riemann tensor and extrinsic curvature are given by

$$
\begin{aligned}
\Gamma_{N P}^{M} & =\frac{1}{2} g^{M K}\left(\partial_{N} g_{K P}+\partial_{P} g_{K M}-\partial_{K} g_{N P}\right), \\
R_{N P Q}^{M} & =\partial_{P} \Gamma_{N Q}^{M}-\partial_{Q} \Gamma_{N P}^{M}+\Gamma_{P K}^{M} \Gamma_{N Q}^{K}-\Gamma_{Q K}^{M} \Gamma_{N P}^{K}, \\
K_{A V} & =h_{A}^{C} \nabla_{C} n_{V}=\frac{1}{2} £_{n} h_{A B},
\end{aligned}
$$

where $£_{n}$ denotes the Lie derivative in direction of $n_{A}$. Finally we can define our model. The action is given by

$$
\begin{aligned}
S= & \frac{1}{16 \pi G} \int d^{5} x \sqrt{-g}\left[R+2 \Lambda-\frac{1}{4} F_{M N} F^{M N}\right. \\
& \left.+\epsilon^{M N P Q R} A_{M}\left(\frac{\kappa}{3} F_{N P} F_{Q R}+\lambda R_{B N P}^{A} R_{A Q R}^{B}\right)\right]+S_{G H}+S_{C S K}, \\
S_{G H}= & \frac{1}{8 \pi G} \int_{\partial} d^{4} x \sqrt{-h} K \\
S_{C S K}= & -\frac{1}{2 \pi G} \int_{\partial} d^{4} x \sqrt{-h} \lambda n_{M} \epsilon^{M N P Q R} A_{N} K_{P L} D_{Q} K_{R}^{L}
\end{aligned}
$$

where $S_{G H}$ is the usual Gibbons-Hawking boundary term and $D_{A} T_{K \ldots}^{S_{K} \ldots}=h_{A}^{B} h_{S_{1}}^{S} h_{K}^{K_{1} \ldots \nabla_{B}} T_{K_{1} \ldots}^{S_{1} \ldots}$ is the covariant derivative acting on an arbitrary tensor $T$ on the four dimensional boundary. The second boundary term $S_{C S K}$ is needed if we want the model to reproduce the gravitational anomaly at general hypersurface. To study the behavior of our model under the relevant gauge and diffeomorphism gauge symmetries we note that the action is diffeomorphism invariant. The Chern Simons terms are well formed volume forms and as such are diffeomorphism invariant. They do depend however explicitly on the gauge connection $A_{M}$. Under gauge transformations $\delta A_{M}=\nabla_{M} \xi$ they are therefore invariant only up to a 
boundary term. We have

$$
\begin{aligned}
\delta S= & \frac{1}{16 \pi G} \int_{\partial} d^{4} x \sqrt{-h} \xi \epsilon^{M N P Q R}\left(\frac{\kappa}{3} n_{M} F_{N P} F_{Q R}+\lambda n_{M} R_{B N P}^{A} R_{A Q R}^{B}\right)- \\
& -\frac{\lambda}{4 \pi G} \int_{\partial} d^{4} x \sqrt{-h} n_{M} \epsilon^{M N P Q R} D_{N} \xi K_{P L} D_{Q} K_{R}^{L} .
\end{aligned}
$$

This is easiest evaluated in Gaussian normal coordinates (see next section) where the metric takes the form $d s^{2}=d r^{2}+\gamma_{i j} d x^{i} d x^{j}$. All the terms depending on the extrinsic curvature cancel thanks to the contributions from $S_{C S K}$. The gauge variation of the action depends only on the intrinsic four dimensional curvature of the boundary and is given by

$$
\delta S=\frac{1}{16 \pi G} \int_{\partial} d^{4} x \sqrt{-h} \epsilon^{m n k l}\left(\frac{\kappa}{3} \hat{F}_{m n} \hat{F}_{k l}+\lambda \hat{R}_{j m n}^{i} \hat{R}^{j}{ }_{i k l}\right) .
$$

This has to be interpreted as the anomalous variation of the effective quantum action of the dual field theory. The anomaly is therefore in the form of the consistent anomaly. Since we are dealing only with a single $U(1)$ symmetry the (gauge) anomaly is automatically expressed in terms of the field strength. We could also express the anomaly in terms of an anomalous current conservation equation. One has to be however careful about the definition of the current since it is always possible to add a Chern-Simons current and redefine $J^{m} \rightarrow J^{m}+c \epsilon^{m n k l} A_{n} F_{k l}$. This redefined current can not be expressed as the variation of a local functional of the fields with respect to the gauge field. In particular the so-called covariant form of the anomaly differs precisely in such a redefinition of the current ${ }^{3}$. In Bertlmann's book 55 the consistent form of the anomaly for chiral fermions transforming under a symmetry group generated by $T_{A}$ is quoted as

$$
D_{m} J_{A}^{m}=\eta_{H} \frac{1}{24 \pi^{2}} \epsilon^{i j k l} \operatorname{Tr}\left[T_{A} \partial_{i}\left(A_{j} \partial_{k} A_{l}+\frac{1}{2} A_{j} A_{k} A_{l}\right)\right],
$$

with $\eta_{H}= \pm$ for $H \in\{R, L\}$ for right-handed and left-handed fermions respectively. We use this to fix $\kappa$ to the anomaly coefficient for a single chiral fermion transforming under a $U(1)_{L}$ symmetry. To do so we simply set $T_{A}=1$ in 5.1.11 which fixes the anomaly coefficient $d_{A B C}=\frac{1}{2} \operatorname{Tr}\left(T_{A}\left\{T_{B}, T_{C}\right\}\right)=1$ and therefore

$$
-\frac{\kappa}{48 \pi G}=\frac{1}{96 \pi^{2}}
$$

Similarly we can fix $\lambda$ by matching to the gravitational anomaly of a single left-handed fermion

and find

$$
D_{m} J^{m}=\frac{1}{768 \pi^{2}} \epsilon^{i j k l} \hat{R}^{m}{ }_{n i j} \hat{R}^{n}{ }_{m k l},
$$

$$
-\frac{\lambda}{16 \pi G}=\frac{1}{768 \pi^{2}}
$$

As a side remark we note that the gravitational anomaly could in principle also be shifted into the diffeomorphism sector. This can be done by adding an additional (Bardeen like) boundary counterterm to the action

$$
S_{c t}=\int d^{4} x \sqrt{-h} A_{m} I^{m}
$$

\footnotetext{
${ }^{3}$ Recall that the effective field theory hydrodynamic approaches following 75 typically use the covariant form of the anomaly 76 .
} 
with $I^{m}=\epsilon^{m n k l}\left(\hat{\Gamma}_{n q}^{p} \partial_{k} \hat{\Gamma}_{l p}^{q}+\frac{2}{3} \hat{\Gamma}_{m p}^{o} \hat{\Gamma}_{k q}^{p} \hat{\Gamma}_{l o}^{q}\right)$ fulfilling $D_{m} I^{m}=\frac{1}{4} \epsilon^{i j k l} \hat{R}^{m}{ }_{n i j} \hat{R}^{n}{ }_{m k l}$. Since this term depends explicitly on the four dimensional Christoffel connection it breaks diffeomorphism invariance.

The bulk equations of motion are

$$
\begin{aligned}
G_{M N}-\Lambda g_{M N} & =\frac{1}{2} F_{M L} F_{N}{ }^{L}-\frac{1}{8} F^{2} g_{M N}+2 \lambda \epsilon_{L P Q R(M} \nabla_{B}\left(F^{P L} R^{B}{ }_{N)}{ }^{Q R}\right)(5 . \\
\nabla_{N} F^{N M} & =-\epsilon^{M N P Q R}\left(\kappa F_{N P} F_{Q R}+\lambda R_{B N P}^{A} R_{A Q R}^{B}\right)
\end{aligned}
$$

and they are gauge and diffeomorphism covariant. We note that keeping all boundary terms in the variations that lead to the bulk equations of motion we end up with boundary terms that contain derivatives of the metric variation normal to the boundary. We will discuss this issue in more detail in the next section where we write down the Gauss-Codazzi decomposition of the action.

\subsection{Kubo formulas, anomalies and Chiral Vortical Conduc- tivity}

The system of equations (5.1.16)-(5.1.17) admit the following exact background AdS Reissner-Nordström black-brane solution with

$$
\begin{aligned}
& d s^{2}=\frac{\bar{r}^{2}}{L^{2}}\left(-f(\bar{r}) d t^{2}+d \vec{x}^{2}\right)+\frac{L^{2}}{\bar{r}^{2} f(\bar{r})} d \bar{r}^{2}, \\
& A^{(0)}=\phi(\bar{r}) d t=\left(\beta-\frac{\mu \bar{r}_{\mathrm{H}}^{2}}{\bar{r}^{2}}\right) d t
\end{aligned}
$$

The blackening factor of the metric is

$$
f(\bar{r})=1-\frac{M L^{2}}{\bar{r}^{4}}+\frac{Q^{2} L^{2}}{\bar{r}^{6}} .
$$

The black brane is located at $\bar{r}=\bar{r}_{\mathrm{H}}$. The parameters $M$ and $Q$ of the RN black hole are related to the chemical potential $\mu$ and the horizon $\bar{r}_{H}$ by

$$
M=\frac{\bar{r}_{\mathrm{H}}^{4}}{L^{2}}+\frac{Q^{2}}{\bar{r}_{\mathrm{H}}^{2}} \quad, \quad Q=\frac{\mu \bar{r}_{\mathrm{H}}^{2}}{\sqrt{3}} .
$$

The Hawking temperature is given in terms of these black hole parameters as

$$
T=\frac{\bar{r}_{\mathrm{H}}^{2}}{4 \pi L^{2}} f\left(\bar{r}_{\mathrm{H}}\right)^{\prime}=\frac{\left(2 \bar{r}_{\mathrm{H}}^{2} M-3 Q^{2}\right)}{2 \pi \bar{r}_{\mathrm{H}}^{5}} .
$$

The pressure of the gauge theory is $P=\frac{M}{16 \pi G L^{3}}$ and its energy density is $\epsilon=3 P$ due to the underlying conformal symmetry.

The AdS/CFT dictionary tells us how to compute the retarded propagators [44, 45]. Since we are interested in studying the linear response limit analytically, we split the metric and gauge field into a background part and a linear perturbation,

$$
\begin{gathered}
g_{M N}=g_{M N}^{(0)}+\epsilon h_{M N}, \\
A_{M}=A_{M}^{(0)}+\epsilon a_{M} .
\end{gathered}
$$

Now we can compute the holographic response functions by applying the prescription of $[2,44,45,51]$. Recall that for a coupled system the holographic computation of the 
correlators consists in finding a maximal set of linearly independent solutions that satisfy infalling boundary conditions on the horizon and that source a single operator at the AdS boundary. Without loss of generality we consider perturbations of momentum $k$ in the $y$-direction at zero frequency. To study the effect of anomalies we just turned on the shear sector (transverse momentum fluctuations) $a_{\alpha}$ and $h_{t}^{\alpha}$, where $\alpha=x, z 4^{4}$ For convenience we redefine new parameters and radial coordinate

$$
\bar{\lambda}=\frac{4 \mu \lambda L}{\bar{r}_{H}^{2}} \quad ; \quad \bar{\kappa}=\frac{4 \mu \kappa L^{3}}{\bar{r}_{H}^{2}} \quad ; \quad a=\frac{\mu^{2} L^{2}}{3 \bar{r}_{\mathrm{H}}^{2}} \quad ; \quad u=\frac{\bar{r}_{H}^{2}}{r^{2}} .
$$

Now the horizon sits at $u=1$ and the AdS boundary at $u=0$. Finally we can write the system of differential equations for the shear sector, that consists on four second order equations. Since we are interested in computing correlators at hydrodynamics regime, we will solve the system up to first order in $k$. The reduced system can be written as

$$
\begin{array}{r}
0=h_{t}^{\alpha^{\prime \prime}}(u)-\frac{h_{t}^{\alpha^{\prime}}(u)}{u}-3 a u B_{\alpha}^{\prime}(u)+i \bar{\lambda} k \epsilon_{\alpha \beta}\left[\left(24 a u^{3}-6(1-f(u))\right) \frac{B_{\beta}(u)}{u}\right. \\
\left.+\left(9 a u^{3}-6(1-f(u))\right) B_{\beta}^{\prime}(u)+2 u\left(u h_{t}^{\beta^{\prime}}(u)\right)^{\prime}\right], \\
0=\quad B_{\alpha}^{\prime \prime}(u)+\frac{f^{\prime}(u)}{f(u)} B_{\alpha}^{\prime}(u)-\frac{h_{t}^{\alpha^{\prime}}(u)}{f(u)} \\
+i k \epsilon_{\alpha \beta}\left(\frac{3}{u f(u)} \bar{\lambda}\left(\frac{2}{a}(f(u)-1)+3 u^{3}\right) h_{t}^{\beta^{\prime}}(u)+\bar{\kappa} \frac{B_{\beta}(u)}{f(u)}\right),
\end{array}
$$

with the gauge field redefined as $B_{\alpha}=a_{\alpha} / \mu$. The complete system of equations depending on frequency and momentum is showed in appendix 7.4. This system consists of six dynamical equations and two constraints.

In order to get solutions at first order in momentum we expand the fields in the dimensionless momentum $p=k / 4 \pi T$ such as

$$
\begin{aligned}
& h_{t}^{\alpha}(u)=h_{t}^{(0) \alpha}(u)+p h_{t}^{(1) \alpha}(u), \\
& B_{\alpha}(u)=B_{\alpha}^{(0)}(u)+p B_{\alpha}^{(1)}(u) .
\end{aligned}
$$

The relevant physical boundary conditions on fields are: $h_{t}^{\alpha}(0)=\tilde{H}^{\alpha}, B_{\alpha}(0)=\tilde{B}_{\alpha}$; where the 'tilde' parameters are the sources of the boundary operators. The second condition compatible with the ingoing one at the horizon is regularity for the gauge field and vanishing for the metric fluctuation [69].

After solving the system perturbatively (see appendix 7.3 for solutions), we can go compute the corresponding holographic Green functions. If we consider the vector of fields to be

$$
\Phi_{k}^{\top}(u)=\left(B_{x}(u), h_{t}^{x}(u), B_{z}(u), h_{t}^{z}(u)\right),
$$

the $\mathcal{A}$ and $\mathcal{B}$ matrices for that setup take the following form

$$
\mathcal{A}=\frac{\bar{r}_{\mathrm{H}}^{4}}{16 \pi G L^{5}} \operatorname{Diag}\left(-3 a f, \frac{1}{u},-3 a f, \frac{1}{u}\right),
$$

\footnotetext{
${ }^{4}$ Since we are in the zero frequency case the fields $h_{y}^{\alpha}$ completely decouple of the system and take a constant value, see appendix 7.4 .
} 


$$
\begin{gathered}
\mathcal{B}_{A d S+\partial}=\frac{\bar{r}_{\mathrm{H}}^{4}}{16 \pi G L^{5}}\left(\begin{array}{cccc}
0 & -3 a & \frac{4 \kappa i k \mu^{2} \phi L^{5}}{3 r_{\mathrm{H}}^{4}} & 0 \\
0 & -\frac{3}{u^{2}} & 0 & 0 \\
\frac{-4 \kappa i k \mu^{2} \phi L^{5}}{3 r_{\mathrm{H}}^{4}} & 0 & 0 & -3 a \\
0 & 0 & 0 & -\frac{3}{u^{2}}
\end{array}\right), \\
\mathcal{B}_{C T}=\frac{\bar{r}_{\mathrm{H}}^{4}}{16 \pi G L^{5}}\left(\begin{array}{ccccc}
0 & 0 & 0 & 0 \\
0 & \frac{3}{u^{2} \sqrt{f}} & 0 & 0 \\
0 & 0 & 0 & 0 \\
0 & 0 & 0 & \frac{3}{u^{2} \sqrt{f}}
\end{array}\right)
\end{gathered}
$$

where $\mathcal{B}=\mathcal{B}_{A d S+\partial}+\mathcal{B}_{C T}$. Notice that there is no contribution to the matrices coming from the Chern-Simons gravity part, the corresponding contributions vanish at the boundary. These matrices and the perturbative solutions are the ingredients to compute the matrix of propagators. Undoing the vector field redefinition introduced in 6.4.104 and 6.4.105) the interesting retarded correlation functions at zero frequency are then

$$
\begin{aligned}
G_{x, z} & =-G_{z, x}=\frac{i \sqrt{3} k Q \kappa}{2 \pi G \bar{r}_{\mathrm{H}}^{2}}+\frac{i k \beta \kappa}{6 \pi G} \\
G_{x, t z} & =G_{t x, z}=-G_{z, t x}=-G_{t z, x}=\frac{3 i k Q^{2} \kappa}{4 \pi G \bar{r}_{\mathrm{H}}^{4}}+\frac{2 i k \lambda \pi T^{2}}{G}, \\
G_{t x, t z} & =-G_{t z, t x}=+\frac{i \sqrt{3} k Q^{3} \kappa}{2 \pi G \bar{r}_{\mathrm{H}}^{6}}+\frac{4 \pi i \sqrt{3} k Q T^{2} \lambda}{G \bar{r}_{\mathrm{H}}^{2}} .
\end{aligned}
$$

Using the Kubo formulae (3.3.59) and (3.3.60) and setting the deformation parameter to zero we recover the conductivities

$$
\begin{aligned}
\sigma_{B} & =-\frac{\sqrt{3} Q \kappa}{2 \pi G \bar{r}_{\mathrm{H}}^{2}}=\frac{\mu}{4 \pi^{2}}, \\
\sigma_{V} & =\sigma_{B}^{\epsilon}=-\frac{3 Q^{2} \kappa}{4 \pi G \bar{r}_{\mathrm{H}}^{4}}-\frac{2 \lambda \pi T^{2}}{G}=\frac{\mu^{2}}{8 \pi^{2}}+\frac{T^{2}}{24}, \\
\sigma_{V}^{\epsilon} & =-\frac{\sqrt{3} Q^{3} \kappa}{2 \pi G \bar{r}_{\mathrm{H}}^{6}}-\frac{4 \pi \sqrt{3} Q T^{2} \lambda}{G \bar{r}_{\mathrm{H}}^{2}}=\frac{\mu^{3}}{12 \pi^{2}}+\frac{\mu T^{2}}{12} .
\end{aligned}
$$

The first expression is in perfect agreement with the literature and the second one shows the extra $T^{2}$ term predicted in $[70]$. In fact the numerical coefficients coincide precisely with the ones obtained in weak coupling. This can be taken as a strong hint that the anomalous conductivities are indeed completely determined by the anomalies and are not renormalized beyond one loop. However, the statement is only true if we do not allow for dynamically generated instanton-type contributions to the (non-)conservation law [REF.] We also point out that the $T^{3}$ term that appears as undetermined integration constant in the hydrodynamic considerations in [124] should make its appearance in $\sigma_{V}^{\epsilon}$. We do not find any such term which is consistent with the argument that this term is absent due to CPT invariance.

Finally let us also note that the shear viscosity is not modified by the presence of the gravitational anomaly. We know that $\eta \propto \lim _{w \rightarrow 0} \frac{1}{w}<T^{x y} T^{x y}>_{k=0}$, so we should solve the system at $k=0$ for the fluctuations $h_{y}^{i}$ but the anomalous coefficients always appear with a momentum $k$ as we can see in $(7.4 .65)$, therefore if we switch off the momentum, the 
system looks precisely as the theory without anomalies. In [125 it has been shown that the black hole entropy does not depend on the extra mixed Chern-Simons term, therefore the shear viscosity entropy ratio remain the same in this mode 5 .

\footnotetext{
${ }^{5}$ For a four dimensional holographic model with gravitational Chern-Simons term and a scalar field this has also been shown in 126 .
} 


\section{Chapter 6}

\section{Cutoff Flow of Anomalous Transport Coefficients in Holography}

One would expect that the remarkable fact that both the CME and the CVC are completely determined by anomalies, which are very robust (topological) objects, prevents these transport coefficients from acquire a dependence on the RG group scale or the cutoff scale, at least in the presence of non-dynamical external fields.

Within the gauge-gravity duality the running with the holographic coordinate can be interpreted as a type of renormalization group (RG) flow in the dual field theory 127 . The first application of this holographic flow to transport coefficients is [128] where it was shown that the electric conductivity and the shear viscosity have a trivial flow. The extension to finite chemical potential has been studied in 129,130 and in 131 the flow is analyzed in the framework of the Gauss-Bonnet theory.

Later on there was a renewed interest in this subject due to the explicit holographic construction of the Wilsonian Renormalization Group [132]. This approach has also served to study in detail the holographic dual of the cutoff scale 133 .

It is natural to analyze the holographic flow of the anomalous conductivities as well. In the work presented below we define a theory equipped with a cutoff $\Lambda$; this means that we are not considering a (holographic) Wilsonian partition function [132], but rather one in which the UV degrees of freedom have been neglected. The interpretation of (6.1.1) as a generating functional of a cutoff theory is present already in the first works on holographic RG 127. The resulting theory is non-local at the scale of the cutoff. Furthermore, studying correlators as functions of $\Lambda$ corresponds to analyzing the value of the Green's functions for different theories, each one equipped with a cutoff. This is why the forthcoming analysis does not correspond to a proper RG flow, but to a cutoff flow. Such a cutoff flow is computed using different approaches associated with equivalent methods; we show that the resulting flow we observed can be easily interpreted as the flow of the chemical potential. 


\subsection{Setup}

Lets show how transport coefficients flow with a variation of the holographic cutoff scale. We define the theory with a cutoff as:

$$
S=\frac{1}{e^{2}} \int_{r<\Lambda} d^{D} x \sqrt{-g}\left(-\frac{1}{4} F_{M N} F^{M N}\right) .
$$

We consider this theory in a general black brane background of the form

$$
d s^{2}=-g_{t t} d t^{2}+g_{r r} d r^{2}+g_{i i} d\left(x^{i}\right)^{2} .
$$

We assume that the above metric has an event horizon at $r=r_{H}$ and that every component depends only on $r$. The boundary is placed at $r=\Lambda$. The metric is also assumed to be regular except at the horizon and possibly in the limit $\Lambda \rightarrow \infty$. The current of the holographic dual field theory is

$$
J^{\mu}=\left.\frac{1}{e^{2}} \sqrt{-g} F^{\mu r}\right|_{r=\Lambda} .
$$

In the gauge $A_{r}=0$ the $x$-component of its variation due to a small perturbation of the gauge field reads

$$
J^{x}=\left.\frac{-1}{e^{2}} \sqrt{-g} g^{x x} g^{r r} \dot{a}(x, r)\right|_{r=\Lambda},
$$

where $\dot{a}=d a / d r$ is the r-derivative of the aforementioned perturbation. We define $a(x, r)$ as $a(x, r)=\frac{a(r)}{a(\Lambda)} a^{(0)}(x)$, so that it is normalized at the boundary to $a(x, \Lambda)=a^{(0)}(x)$ and $a(r)$ solves the radial wave equation

$$
\ddot{a}(r)+\frac{1}{2} \dot{a}(r)\left(g^{t t} \dot{g}_{t t}+g^{x x} \dot{g}_{x x}-g^{r r} \dot{g}_{r r}\right)+g_{r r}\left(\omega^{2} g^{t t}-k^{2} g^{x x}\right) a(r)=0 .
$$

On the other hand, we define the electric conductivity at the boundary as $J^{x}=\sigma_{E}(\Lambda) E(\Lambda)$, where $E(\Lambda)=-i \omega a^{(0)}$ is the external applied electric field. Comparing this to equation (6.1.4) we conclude

$$
\sigma_{E}(\Lambda)=\left.\frac{-i}{e^{2} \omega} \sqrt{-g} g^{x x} g^{r r} \frac{\dot{a}(r)}{a(\Lambda)}\right|_{r=\Lambda} .
$$

Varying the cutoff $\Lambda \rightarrow \Lambda+d \Lambda$ we find the for the differential of the electric conductivity

$$
\frac{d \sigma_{E}(\Lambda)}{d \Lambda}=\frac{-i}{e^{2} \omega}\left[\frac{d}{d r}\left(\sqrt{-g} g^{x x} g^{r r} \frac{d a(r) / d r}{a(r)}\right)\right]_{r=\Lambda} .
$$

This equation shows that we can study the flow of the transport coefficients with the cutoff reformulating it as the evolution with respect to the coordinate $r$, by formally identifying $r$ with $\Lambda$.

We can use now the equation of motion for the perturbation $a(r)$ and the definition of the conductivity (6.1.6) to derive the flow equation

$$
\frac{d \sigma_{E}(\omega, k)}{d \Lambda}=-i \omega\left[\frac{e^{2}}{\sqrt{-g}} g_{r r} g_{x x} \sigma_{E}^{2}+\frac{\sqrt{-g}}{e^{2}} g^{x x}\left(g^{t t}+\frac{k^{2}}{\omega^{2}} g^{x x}\right)\right]
$$

This the flow equation first derived in [128]. It can be solved by demanding infalling boundary conditions on the horizon. In particular the flow for the DC conductivity turns out to be trivial $\dot{\sigma}_{E}=0$. In this case the electric conductivity is completely determined by its value on the horizon via the membrane paradigm

$$
\sigma_{E}(\Lambda)=\sigma_{E}\left(r_{H}\right)=\frac{1}{e^{2}} .
$$




\subsection{Flow of anomalous conductivities}

We will apply now the strategy outlined before to the anomalous transport coefficients. Two models will be considered. First we discuss a model in which we neglect the backreaction of the gauge field fluctuations on the metric. We will study the interplay between two $U(1)$ symmetries which we call vector and axial ones. This allows to model the chiral magnetic and the chiral separation effect. A second model will use only one anomalous $U(1)$ symmetry but we will also include the backreaction onto the metric. This allows to study also the flow of the chiral vortical conductivity and the flow of the anomalous transport coefficients related to the energy current.

\subsubsection{Vector and Axial symmetries}

We will apply the aforementioned strategy to the chiral magnetic conductivity . Its proper definition requires the interplay between a vector like $U(1)$ symmetry and an axial $U(1)$ symmetry. Holographic models have been investigated in [85, 134, 135]. The model allows for the definition of the chiral magnetic conductivity and axial conductivities involving external axial magnetic fields. Its action is given by 135

$S=\int \sqrt{-g}\left(-\frac{1}{4 g_{V}^{2}} F_{M N}^{V} F_{V}^{M N}-\frac{1}{4 g_{A}^{2}} F_{M N}^{A} F_{A}^{M N}+\frac{\kappa}{2} \epsilon^{M N P Q R} A_{M}\left(F_{N P}^{A} F_{Q R}^{A}+3 F_{N P}^{V} F_{Q R}^{V}\right)\right)$

where $V$ stands for "vector" and $A$ for "axial". The Lagrangian contains two Maxwell actions for vector and axial gauge fields and a particular choice of Chern-Simons term. In what follows, we will stick to the notation of the previous chapter; concretely, we define the epsilon symbol as $\epsilon(A B C D E)=-\sqrt{-g} \epsilon^{A B C D E}$, with $\epsilon(r t x y z)=1$ ( $r$ corresponds to the fifth coordinate). From the boundary term of this action, after perturbing both the axial and the vector gauge fields, we obtain an expression for the boundary theory currents

$$
\begin{aligned}
& J^{\mu}=\left.\left(\frac{1}{g_{V}^{2}} \sqrt{-g} F_{V}^{\mu r}+6 \kappa \epsilon^{\mu \nu \rho \lambda} A_{\nu} F_{\rho \lambda}^{V}\right)\right|_{r=\Lambda}, \\
& J_{5}^{\mu}=\left.\left(\frac{1}{g_{A}^{2}} \sqrt{-g} F_{A}^{\mu r}+2 \kappa \epsilon^{\mu \nu \rho \lambda} A_{\nu} F_{\rho \lambda}^{A}\right)\right|_{r=\Lambda},
\end{aligned}
$$

where $\epsilon^{\mu \nu \rho \lambda} \equiv \epsilon^{r \mu \nu \rho \lambda}$. The coefficients in front of the Chern-Simons terms are crucial to ensure that the vector current is non-anomalous $D_{\mu} J_{V}^{\mu}=0$. The axial current is anomalous $D_{\mu} J_{5}^{\mu}=-\frac{\kappa}{2} \epsilon^{\mu \nu \rho \lambda}\left(3 F_{\mu \nu}^{V} F_{\rho \lambda}^{V}+F_{\mu \nu}^{A} F_{\rho \lambda}^{A}\right)$ 135. Comparing with the standard result from the one loop triangle calculation, we find $\kappa=-\frac{N_{c}}{24 \pi^{2}}$ for a dual strongly coupled $S U\left(N_{c}\right)$ gauge theory for a mass less Dirac fermion in the fundamental representation. Note also that both currents are invariant under vector gauge transformations but not under axial gauge transformations.

The equations of motion for the gauge fields are

$$
\begin{aligned}
\frac{1}{g_{A}^{2}} \nabla_{N} F_{A}^{N M}+\frac{3 \kappa}{2} \epsilon^{M N P Q R}\left(F_{N P}^{A} F_{Q R}^{A}+F_{N P}^{V} F_{Q R}^{V}\right) & =0, \\
\frac{1}{g_{V}^{2}} \nabla_{N} F_{V}^{N M}+3 \kappa \epsilon^{M N P Q R}\left(F_{N P}^{A} F_{Q R}^{V}\right) & =0 .
\end{aligned}
$$

In order to study the flow of the conductivities with the fifth coordinate, we will proceed as follows: 
- We introduce an axial and vector perturbation of the gauge fields

$$
\begin{aligned}
& A_{M}=A_{M}^{(0)}+a_{M}(y, t, r), \\
& V_{M}=V_{M}^{(0)}+v_{M}(y, t, t) .
\end{aligned}
$$

We switch perturbations on only in the $z$ and $x$-directions (transverse directions): $a_{z}(y, t, r), v_{z}(y, t, r), a_{x}(y, t, r), v_{x}(y, t, r)$

- Since the Chern-Simons contribution to the current depends only on the intrinsic gauge fields on the cutoff surface, its flow is trivial. The non-trivial part of a possible flow is completely contained in the covariant currents

$$
\begin{aligned}
& J^{(1) x}=\left.\left(\frac{1}{g_{V}^{2}} \sqrt{-g} F_{V}^{(1) x r}\right)\right|_{r=\Lambda}, \\
& J_{5}^{(1) x}=\left.\left(\frac{1}{g_{A}^{2}} \sqrt{-g} F_{A}^{(1) x r}\right)\right|_{r=\Lambda} .
\end{aligned}
$$

- We define our transport coefficients as the response to the perturbations and in terms of the previously defined covariant currents as

$$
\begin{aligned}
& J^{(1) x}=\sigma_{C M E} \epsilon(r t x y z) F_{y z}^{(1) V}+\sigma_{\text {axial }} \epsilon(r t x y z) F_{y z}^{(1) A}, \\
& J_{5}^{(1) x}=\sigma_{\text {axial }} \epsilon(r t x y z) F_{y z}^{(1) V}+\sigma_{55} \epsilon(r t x y z) F_{y z}^{(1) A} .
\end{aligned}
$$

$\sigma_{\text {axial }}$ defines the vector current generated by an external axial magnetic field. Observe that, in order not to have $F_{\{A, V\}}^{(1) x r}=0$ identically, one has to turn on the perturbations $a_{x}(y, t, r), v_{x}(y, t, r)$. However, these do not play a role when studying the flow of the anomalous conductivities for they induce contributions that tend to zero in the low $\omega$, low $k$ limit, very much as it occurs in [128].

The value of the background gauge fields is 135

$$
\begin{gathered}
A_{0}^{(0)}=\alpha-\frac{\mu_{5} r_{H}^{2}}{r^{2}}, \\
V_{0}^{(0)}=\gamma-\frac{\mu r_{H}^{2}}{r^{2}} .
\end{gathered}
$$

The integration constants $\alpha$ and $\gamma$ can be fixed by e.g. demanding that the gauge fields vanish on the horizon. In any case the covariant currents do not depend on these integration constants. The consistent currents (6.2.11), 6.2.12) do however depend on them through the Chern-Simons currents. For a discussion of this dependence see [134, 135].

The procedure consists of using the equations of motion to find the value of $\partial_{r} \sigma$, where $\sigma$ a generic conductivity defined at some hyper surface $r=\Lambda$. In fact, we only need the equations of motion projected onto $x$ and the Bianchi identity associated with the indices $(r, y, z)$ to obtain an expression of the derivative with respect to $r$ of the different transport coefficients. From the simple form of our metric it can be seen that the vector normal to a hyper surface of $x=$ constant reads $n_{\mu}^{x}=\sqrt{g_{x x}}(0,0,1,0,0)$. Hence, for the vector gauge field we have

$$
n_{M}^{x}\left[\frac{1}{g_{V}^{2}} \nabla_{N} F_{V}^{N M}+3 \kappa \epsilon^{M N P Q R}\left(F_{N P}^{A} F_{Q R}^{V}\right)\right]=0 .
$$


Taking advantage of the relation $\nabla_{N} F^{N M}=\frac{1}{\sqrt{-g}} \partial_{N}\left(\sqrt{-g} F^{N M}\right)$ and the definition of the currents (6.2.17) and (6.2.18), we arrive at

$$
\partial_{r} J^{(1) x}=-12 \kappa \sqrt{-g} \epsilon^{r t x y z}\left(F_{t r}^{A(0)} F_{y z}^{V(1)}+F_{y z}^{A(1)} F_{t r}^{V(0)}\right),
$$

where we have neglected $F_{V}^{(1) t x} ; F_{V}^{(1) y x}$ for these modes lead to vanishing contributions in the low momentum and low frequency limit, as mentioned before. Besides, we have carried out the contraction $\epsilon^{x N P Q R} F_{N P}^{A} F_{Q R}^{V}=-4 \epsilon^{r t x y z}\left(F_{t r}^{A} F_{y z}^{V}+F_{y t}^{A} F_{r z}^{V}+F_{t z}^{A} F_{r y}^{V}+(A \leftrightarrow V)\right)$. The Bianchi identity to first order associated with indices $(r, y, z)$ reads

$$
\partial_{r} F_{y z}^{\{V, A\}(1)}+\partial_{y} F_{z r}^{\{V, A\}(1)}+\partial_{z} F_{r y}^{\{V, A\}(1)}=0 .
$$

Assuming $\partial_{z} F_{r y}^{\{V, A\}(1)} \sim g_{r r} g_{y y} \partial_{z} J^{y(1)}=0$ we obtain

$$
\partial_{r} F_{y z}^{\{V, A\}(1)}=-\frac{g_{z z} g_{r r}}{\sqrt{-g}} g_{\{V, A\}}^{2} \partial_{y} J_{\{V, A\}}^{(1) z} .
$$

Now, making use of these ingredients, the computation of $\partial_{r} \sigma$ is immediate:

$$
\partial_{r} \sigma_{C M E}=\lim _{\omega, k \rightarrow 0}\left[\frac{\partial_{r} J^{x(1)}}{\epsilon(r t x y z) F_{y z}^{V(1)}}-\frac{J^{x(1)}}{\left(\epsilon(r t x y z) F_{y z}^{V(1)}\right)^{2}} \partial_{r} F_{y z}^{V(1)}\right]_{a_{M}=0}
$$

Plugging (6.2.24) and 6.2.26) into (6.2.27) we find, in momentum space

$$
\partial_{r} \sigma_{C M E}=\lim _{\omega, k \rightarrow 0}\left[12 \kappa F_{t r}^{A(0)}+i k \sigma_{C M E} \frac{g_{z z} g_{r r}}{\sqrt{-g}} g_{V}^{2} \frac{J^{(1) z}}{\epsilon(r t x y z) F_{y z}^{V(1)}}\right]
$$

Taking the limit $\omega, k \rightarrow 0$ and substituting $F_{t r}^{V(0)}=-\partial_{r} A_{0}^{(0)}=-2 \frac{\mu_{5} r_{H}^{2}}{r^{3}}$, we get the following flow equation for the chiral magnetic conductivity

$$
\partial_{r} \sigma_{C M E}=-24 \kappa \frac{\mu_{5} r_{H}}{r^{3}}
$$

whose solution is

$$
\sigma_{C M E}=C+12 \kappa \frac{\mu_{5} r_{H}^{2}}{r^{2}} .
$$

Here, $C$ is an integration constant that we must fix. In order to do that, we impose in-falling boundary conditions for the perturbations (or, equivalently, regularity at the horizon $r=r_{H}$ [135). This in turn implies that the fields must depend only on the combination $d v=d t+\sqrt{\frac{g_{r r}}{g_{t t}}} d r 128$. Therefore, in the $A_{r}=0$ gauge, we have

$$
\partial_{r} A_{x}=\sqrt{\frac{g_{r r}}{g_{t t}}} \partial_{t} A_{x} \text { at } r=r_{H}
$$

This condition forces directly $J^{(1) x}\left(r=r_{H}\right)$ to be

$$
j^{(1) x}\left(r=r_{H}\right) \sim F^{(1) x r}\left(r=r_{H}\right) \sim E^{i}
$$


Imposing these infalling boundary conditions results therefore in a vanishing chiral magnetic conductivity at the horizon for the covariant current 11 Thus the integration constant $C$ can be fixed simply by the condition ${ }^{2}$

$$
\sigma_{C M E}\left(r=r_{H}\right)=0 \rightarrow C=-12 \kappa \mu_{5}=\frac{N_{c} \mu_{5}}{2 \pi^{2}} .
$$

The transport coefficients 6.2 .19$)$ and 6.2 .20 can be calculated in an analogous way. For the axial current the projected equation is

$$
n_{x M}\left[\frac{1}{g_{A}^{2}} \nabla_{N} F_{A}^{N M}+\frac{3 \kappa}{2} \epsilon^{M N P Q R}\left(F_{N P}^{A} F_{Q R}^{A}+F_{N P}^{V} F_{Q R}^{V}\right)\right]=0
$$

which implies

$$
\partial_{r} j_{5}^{(1) x}=\sqrt{-g} 12 \kappa \epsilon^{r t x y z}\left(\frac{2 \mu_{5}}{r^{3}} F_{y z}^{(1) A}+\frac{2 \mu}{r^{3}} F_{y z}^{(1) V}\right)
$$

The values of the conductivities at $r=\Lambda$ then read

$$
\begin{array}{r}
\sigma_{C M E}(\Lambda)=\frac{N_{c} \mu_{5}}{2 \pi^{2}}\left(1-\frac{r_{H}^{2}}{\Lambda^{2}}\right) \\
\sigma_{\text {axial }}(\Lambda)=\frac{N_{c} \mu}{2 \pi^{2}}\left(1-\frac{r_{H}^{2}}{\Lambda^{2}}\right) \\
\sigma_{55}(\Lambda)=\frac{N_{c} \mu_{5}}{2 \pi^{2}}\left(1-\frac{r_{H}^{2}}{\Lambda^{2}}\right)
\end{array}
$$

As expected, the result in the limit $r \rightarrow \infty$ is precisely the one obtained in 135] using AdS/CFT techniques. In view of the topological nature and the non-renormalization theorem for the chiral magnetic conductivity it is at first sight somewhat surprising to find a non-trivial flow. This flow becomes however natural if we define the chemical potential in its elementary way as the energy needed to introduce one unit of charge into the ensemble. In the holographic dual this corresponds to bring a unit of charge from the boundary, now situated at $r=\Lambda$ behind the horizon. The energy difference between a unit of charge at the boundary and a unit of charge at the horizon is just given by $A_{0}(\Lambda)-A_{0}\left(r_{H}\right)=\mu(\Lambda)$. This defines an effective chemical potential in the theory equipped with the cutoff $\Lambda$. In fact the definition of such an effective chemical potential is natural even in field theory. If we have a momentum cutoff of order $\Lambda$ we can localize a unit charge only inside a volume within a radius of order $1 / \Lambda$. Thermalizing this unit of charge means spreading it out over the entire ensemble. The difference in energy between the two configurations, the unit of charge localized within $1 / \Lambda$ and spread out over the ensemble again is the effective chemical potential. All the anomalous conductivities can therefore be expressed in the form

$$
\sigma(\Lambda)=\frac{N_{c} \mu(\Lambda)}{2 \pi^{2}}
$$

The are linear in the chemical potential and the numerical coefficient is independent of the cutoff. In this sense they obey the expected non-renormalization theorem.

\footnotetext{
${ }^{1}$ Note that the consistent currents might have non-vanishing chiral magnetic conductivity on the horizon due to the Chern-Simons contribution and depending on the value of the integration constant $\alpha$.

${ }^{2}$ Recall that $\kappa=-\frac{N_{c}}{24 \pi^{2}}$.
} 


\subsubsection{Inclusion of metric perturbations}

In this section we compute the flow equations for the Green's functions associated with generic response. The method can be described as follows: we need to consider two equations. One is the constitutive equation

$$
\left\langle\mathcal{O}_{j}\right\rangle=\sum_{i}^{N} G_{j}^{i} \phi_{i}
$$

and the other one is the covariant holographic definition of the one-point functions, evaluated on some perturbed state. Generically, these would be a functional of the perturbations and its derivatives (the dot means $d / d r$. We will be using both notations indistinctly).

$$
\left\langle\mathcal{O}_{j}\right\rangle=-\sum_{i}^{N}\left(\mathcal{F}_{j}^{i} \phi_{i}+\mathcal{H}_{j}^{i} \dot{\phi}_{i}\right)
$$

Taking the r-derivative in both equations, we can force them to be equal. Observe that, from 6.2.41, we expect terms containing $\mathcal{H}_{j}^{i} \ddot{\phi}_{i}$. After using the equations of motion, we will be left with some expression involving only $\phi$ and $\dot{\phi}$. Then, by equating 6.2.40 and 6.2.41, it is possible to find a formula for $\dot{\phi}_{j}=\sum_{i}^{N} K_{j}^{i} \phi_{i}$ so that eventually we are able to write the r-derivative of (6.2.41) as an expansion in the perturbations only.

On the other hand, differentiating 6.2.40 and using again $\dot{\phi}_{j}=\sum_{i}^{N} K_{j}^{i} \phi_{i}$, we are lead to an expression in terms of $G_{j}^{i}, \dot{G}_{j}^{i}$ and $\phi_{i}$. Imposing that the r-derivative of 6.2.41 and that of 6.2.40) are identical, we finally arrive at

$$
0=\sum_{i}^{N} A_{j}^{i} \phi_{i}
$$

where $A_{j}^{i}$ is a functional of $G_{j}^{i}$ and their first derivatives. Assuming now that the different perturbations are independent from each other, we get $N$ independent equations

$$
A_{j}^{i}=0
$$

which are nothing but differential equations for $G_{j}^{i}$. Remarkable enough, the flow equations for the retarded correlators are of first order in r-derivatives.

\subsubsection{Application to the anomalous conductivities}

In what follows we will derive the flow equations in the presence of a pure gauge ChernSimons term (no gravitational anomaly) by using the procedure detailed in the previous section. The model reads

$$
S=S_{E H}+S_{G H}+\frac{1}{16 \pi G} \int_{r<\Lambda} \sqrt{-g}\left(-\frac{1}{4} F_{M N} F^{M N}+\frac{\kappa}{3} \epsilon^{M N P Q R} A_{M} F_{N P} F_{Q R}\right)
$$

where $S_{E H}$ denotes the Einstein-Hilbert action with negative cosmological constant and $S_{G H}$ is the Gibbons-Hawking term on the boundary $r=\Lambda$. The Chern-Simons coupling is here related to the anomaly for a single chiral fermion by $\kappa=-G /(2 \pi)$. Since we need now the precise equations of motion for the metric fluctuations we will specialize the analysis to a Reissner-Nordstrom AdS Black Brane

$$
\begin{array}{r}
d s^{2}=\frac{r^{2}}{L^{2}}\left(-f(r) d t^{2}+d \vec{x}^{2}\right)+\frac{L^{2}}{r^{2} f(r)} d r^{2} \\
A^{(0)}=\phi(r) d t=-\frac{\mu r_{H}^{2}}{r^{2}}
\end{array}
$$


The integration constant in the gauge field is set that it vanishes for $r \rightarrow \infty$. The horizon of the black hole is located at $r=r_{H}$ and the blackening factor is $f(r)=1-\frac{M L^{2}}{r^{4}}+\frac{Q^{2} L^{2}}{r^{6}}$. The parameters $M, Q$ are related to the chemical potential at infinity $\mu$ and $r_{H}$ by $M=\frac{r_{H}^{4}}{L^{2}}+\frac{Q^{2}}{r_{H}^{2}}$, $Q=\frac{\mu r_{H}^{2}}{\sqrt{3}}$. Finally, the Hawking temperature is given by

$$
T=\frac{r_{H}^{2}}{4 \pi L^{2}} \dot{f}\left(r_{H}\right)=\frac{2 r_{H}^{2} M-3 Q^{2}}{2 \pi r_{H}^{5}}
$$

In what follows, we consider perturbations of momentum $k$ in the $y$-direction at zero frequency. It is only necessary to turn on the shear sector, that is, the perturbations are written as $a_{\alpha}, h_{t}^{\alpha}$, where $\alpha=x, z 3^{3}$. It is more convenient to work with the coordinate $u=\frac{r_{H}^{2}}{r^{2}}$ instead of $r$.

The equations of motion for the perturbations derived from 6.2.44, when $\omega=0$ and to $\mathcal{O}(k)$, read

$$
\begin{aligned}
& 0=B_{\alpha}^{\prime \prime}(u)+\frac{f^{\prime}(u)}{f(u)} B_{\alpha}^{\prime}(u)-\frac{h_{t}^{\alpha^{\prime}}(u)}{f(u)}+i k \epsilon_{\alpha \beta} \bar{\kappa} \frac{B_{\beta}(u)}{f(u)}, \\
& 0=h_{t}^{\alpha^{\prime \prime}}(u)-\frac{h_{t}^{\alpha^{\prime}}(u)}{u}-3 a u B_{\alpha}^{\prime}(u)
\end{aligned}
$$

where $\bar{\kappa}=\frac{4 \mu \kappa L^{3}}{r_{H}^{2}}$. The operators that we will be working with have the following form when evaluated in a perturbed state (for further details see [69])

$$
\begin{aligned}
\delta J^{\alpha} & =\frac{r_{H}^{2}}{8 \pi G L^{3}}\left(f(u) a_{\alpha}^{\prime}-\mu h_{t}^{\alpha}\right) \\
\delta t_{t}^{\alpha} & =\frac{r_{H}^{4} f(u)}{8 \pi G L^{5} u}\left(h_{t}^{\prime \alpha}-\frac{3}{u} h_{t}^{\alpha}\right)
\end{aligned}
$$

where the prime stands for $d / d u$. Differentiating 6.2.50 and 6.2.51) we are left with

$$
\begin{aligned}
\left(\delta J^{\alpha}\right)^{\prime} & =\frac{r_{H}^{2}}{8 \pi G L^{3}}\left(a_{\alpha}^{\prime \prime}(u) f(u)+a_{\alpha}^{\prime}(u) f^{\prime}(u)-\mu h_{t}^{\prime \alpha}\right), \\
\left(\delta t_{t}^{\alpha}\right)^{\prime} & =\frac{r_{H}^{4} f(u)}{8 \pi G L^{5} u}\left(h_{t}^{\prime \prime \alpha}+h_{t}^{\prime \alpha}\left[\frac{f^{\prime}(u)}{f(u)}-\frac{4}{u}\right]+h_{t}^{\alpha}\left[\frac{6}{u^{2}}-\frac{3 f^{\prime}(u)}{u f(u)}\right]\right) .
\end{aligned}
$$

In order to handle the $\phi_{i}^{\prime \prime}$ terms, we evaluate the above expressions on-shell, yielding

$$
\begin{aligned}
\left(\delta J^{\alpha}\right)^{\prime} & =-\frac{r_{H}^{2}}{8 \pi G L^{3}} \bar{\kappa} i k \epsilon_{\alpha \beta} a_{\beta} \\
\left(\delta t_{t}^{\alpha}\right)^{\prime} & =\frac{r_{H}^{4} f(u)}{8 \pi G L^{5} u}\left(h_{t}^{\prime \alpha}\left[\frac{f^{\prime}(u)}{f(u)}-\frac{3}{u}\right]+h_{t}^{\alpha}\left[\frac{6}{u^{2}}-\frac{3 f^{\prime}(u)}{u f(u)}\right]+\frac{3 a u}{\mu} a_{\alpha}^{\prime}\right)
\end{aligned}
$$

Now, observe that, since $h_{t}^{\alpha}=\frac{8 \pi G L^{5} u}{r_{H}^{4} f(u)} \delta t_{t}^{\alpha}+\frac{3}{u} h_{t}^{\alpha}$ and $a_{\alpha}^{\prime}=\left(\frac{8 \pi G L^{3}}{r_{H}^{2}} \delta J^{\alpha}+\mu h_{t}^{\alpha}\right) \frac{1}{f(u)}$, equation 6.2.55 turns into

$$
\left(\delta t_{t}^{\alpha}\right)^{\prime}=\frac{3 r_{H}^{4} f(u)}{8 \pi G L^{5} u}\left[\frac{a u}{f(u)}-\frac{1}{u^{2}}\right] h_{t}^{\alpha}+\left[\frac{f^{\prime}(u)}{f(u)}-\frac{3}{u}\right] \delta t_{t}^{\alpha}+\frac{3 r_{H}^{2} a}{L^{2} \mu} \delta J^{\alpha}
$$

\footnotetext{
${ }^{3}$ At zero frequency the fields $h_{y}^{\alpha}$ decouple from the system and thus will not be considered (see the previous chapter).
} 
Plugging the constitutive relations $\left(\epsilon^{x z} \equiv 1\right)$

$$
\begin{aligned}
\delta J_{\text {const }}^{\alpha} & =G^{x x} \delta^{\alpha \beta} a_{\beta}+G^{x z} \epsilon^{\alpha \beta} a_{\beta}+P^{x t} \delta^{\alpha \beta} h_{\beta}^{t}+P^{z t} \epsilon^{\alpha \beta} h_{\beta}^{t} \\
\delta t_{\text {const }}^{\alpha} & =G_{\epsilon}^{x x} \delta^{\alpha \beta} a_{\beta}+G_{\epsilon}^{x z} \epsilon^{\alpha \beta} a_{\beta}+P_{\epsilon}^{x t} \delta^{\alpha \beta} h_{\beta}^{t}+P_{\epsilon}^{z t} \epsilon^{\alpha \beta} h_{\beta}^{t}
\end{aligned}
$$

into 6.2 .56 , the remaining equation for $\left(\delta t_{t}^{\alpha}\right)^{\prime}$ involves only $\phi_{i}$ and $G_{j}^{i}$.

On the other hand, we can take the u-derivative of (6.2.57)- 6.2.58) explicitly and then make use of 6.2.50- 6.2.51 to end up having an equation in terms of $\phi_{i}, G_{j}^{i}$ and $G_{j}^{i}$. Finally, imposing $\left(\delta J_{\text {const }}^{\alpha}\right)^{\prime}=\left(\delta J^{\alpha}\right)^{\prime}$ and $\left(\delta t_{t \text { const }}^{\alpha}\right)^{\prime}=\left(\delta t_{t}^{\alpha}\right)^{\prime}$ and assuming that the perturbations $\phi_{i}$ are independent from each other, we find

$$
\begin{aligned}
& G^{\prime x x}+\frac{8 \pi G L^{3}}{f(u) r_{H}^{2}}\left(\left(G^{x x}\right)^{2}-\left(G^{x z}\right)^{2}\right)-\frac{8 \pi G L^{5} u}{r_{H}^{4} f^{2}(u)}\left(P^{x t} G_{\epsilon}^{x x}-P^{z t} G_{\epsilon}^{x z}\right)=0 \\
& G^{\prime x z}+\frac{16 \pi G L^{3}}{f(u) r_{H}^{2}} G^{x x} G^{x z}-\frac{8 \pi G L^{5} u}{r_{H}^{4} f^{2}(u)}\left(P^{x t} G_{\epsilon}^{x z}+P^{z t} G_{\epsilon}^{x x}\right)=-\frac{r_{H}^{2}}{8 \pi G L^{3}} \bar{\kappa} i k \\
& P^{\prime x t}+G^{x x}\left(-\mu+\frac{8 \pi G L^{3}}{f(u) r_{H}^{2}} P^{x t}\right)-\left(\frac{8 \pi G L^{3}}{f(u) r_{H}^{2}} G^{x z}-\frac{8 \pi G L^{5} u}{r_{H}^{4} f^{2}(u)} P_{\epsilon}^{z t}\right) P^{z t}+ \\
& +P^{x t}\left(-\frac{8 \pi G L^{5} u}{r_{H}^{4} f^{2}(u)} P_{\epsilon}^{x t}-\frac{f^{\prime}(u)}{f(u)}+\frac{3}{u}\right)=0 \\
& P^{\prime z t}+G^{x z}\left(-\mu+\frac{8 \pi G L^{3}}{f(u) r_{H}^{2}} P^{x t}\right)-\frac{8 \pi G L^{5} u}{f^{2}(u) r_{H}^{4}} P^{x t} P_{\epsilon}^{z t}+ \\
& +P^{z t}\left(-\frac{8 \pi G L^{5} u}{f^{2}(u) r_{H}^{4}} P_{\epsilon}^{x t}+\frac{3}{u}-\frac{f^{\prime}(u)}{f(u)}+\frac{8 \pi G L^{3}}{f(u) r_{H}^{2}} G^{x x}\right)=0 \\
& G_{\epsilon}^{\prime x x}+\frac{8 \pi G L^{3}}{f(u) r_{H}^{2}}\left(G_{\epsilon}^{x x} G^{x x}-G_{\epsilon}^{x z} G^{x z}\right)-\frac{8 \pi G L^{5} u}{r_{H}^{4} f^{2}(u)} P_{\epsilon}^{x t} G_{\epsilon}^{x x}+\frac{8 \pi G L^{5} u}{r_{H}^{4} f^{2}(u)} P_{\epsilon}^{z t} G_{\epsilon}^{x z}= \\
& =-G_{\epsilon}^{x x}\left(\frac{3}{u}-\frac{f^{\prime}(u)}{f(u)}\right)+\mu G^{x x} \\
& G_{\epsilon}^{\prime x z}+\frac{8 \pi G L^{3}}{f(u) r_{H}^{2}}\left(G_{\epsilon}^{x x} G^{x z}+G_{\epsilon}^{x z} G^{x x}\right)-\frac{8 \pi G L^{5} u}{r_{H}^{4} f^{2}(u)}\left(P_{\epsilon}^{x t} G_{\epsilon}^{x z}+P_{\epsilon}^{z t} G_{\epsilon}^{x x}\right)= \\
& =-G_{\epsilon}^{x z}\left(\frac{3}{u}-\frac{f^{\prime}(u)}{f(u)}\right)+\mu G^{x z} \\
& P_{\epsilon}^{\prime x t}+G_{\epsilon}^{x x}\left(-\mu+\frac{8 \pi G L^{3}}{f(u) r_{H}^{2}} P^{x t}\right)+P_{\epsilon}^{x t}\left(-\frac{8 \pi G L^{5} u}{r_{H}^{4} f^{2}(u)} P_{\epsilon}^{x t}+\frac{3}{u}-\frac{f^{\prime}(u)}{f(u)}\right)+ \\
& -\frac{8 \pi G L^{3}}{f(u) r_{H}^{2}} G_{\epsilon}^{x z} P^{z t}+\frac{8 \pi G L^{5} u}{r_{H}^{4} f^{2}(u)}\left(P_{\epsilon}^{z t}\right)^{2}=-P_{\epsilon}^{x t}\left(\frac{3}{u}-\frac{f^{\prime}(u)}{f(u)}\right)+ \\
& +\mu P^{x t}-\frac{3 r_{H}^{4}}{8 \pi G L^{5} u} f(u)\left(a u-\frac{f(u)}{u^{2}}\right) \\
& P_{\epsilon}^{\prime z t}+G_{\epsilon}^{x z}\left(\frac{8 \pi G L^{3}}{f(u) r_{H}^{2}} P^{x t}-\mu\right)+\frac{8 \pi G L^{3}}{f(u) r_{H}^{2}} G_{\epsilon}^{x x} P^{z t}-\frac{8 \pi G L^{5} u}{f^{2}(u) r_{H}^{4}} P_{\epsilon}^{x t} P_{\epsilon}^{z t}+ \\
& +P_{\epsilon}^{z t}\left(-\frac{8 \pi G L^{5} u}{f^{2}(u) r_{H}^{4}} P_{\epsilon}^{x t}+\frac{3}{u}-\frac{f^{\prime}(u)}{f(u)}\right)=-P_{\epsilon}^{z t}\left(\frac{3}{u}-\frac{f^{\prime}(u)}{f(u)}\right)+\mu P^{z t}
\end{aligned}
$$

By directly studying the structure of the solutions to 6.2 .48$)-(6.2 .49)$, it can be realized that $G^{x x}=P^{x t}=G_{\epsilon}^{x x}=0$ for $\omega=0$ and to first order in $k$. Furthermore, all the anomalous correlators are of order $k$ or higher. A more detailed study of 6.2.59-6.2.66 is left for section 6.3.1, 


\subsection{Flow of the transport coefficients as two point functions}

As suggested in Section 6.2, we could have determined the flow by simply considering the system to be restricted to live between the horizon and a cutoff surface placed at $\Lambda$. It is hence expected that the transport coefficients at the boundary can be computed by finding the corresponding 2-point functions. The boundary value of the perturbations, whose bulk-to-boundary propagator is normalized at the cutoff, work as the sources for the different operators of the dual theory.

Henceforth, the perturbations will be rearranged in a vector $\Phi\left(u, x^{\mu}\right)$. It is more convenient to use the Fourier transformed quantity

$$
\Phi\left(u, x^{\mu}\right)=\int \frac{d^{d} k}{(2 \pi)^{d}} \Phi_{k}^{I}(u) e^{-i \omega t+i \vec{k} \vec{x}}
$$

The explicit expression for $\Phi_{k}(u)$ is

$$
\Phi_{k}^{\top}(u)=\left(B_{x}(u), h_{t}^{x}(u), B_{z}(u), h_{t}^{z}(u)\right)
$$

being $B_{\alpha}=a_{\alpha} / \mu$. To proceed, one can follow 51 and assume the general form of a boundary action

$$
\delta S^{(2)}=\int_{r=\Lambda} \frac{d^{d} k}{(2 \pi)^{d}}\left[\Phi_{-k}^{I} \mathcal{A}_{I J} \Phi_{k}^{\prime J}+\Phi_{-k}^{I} \mathcal{B}_{I J} \Phi_{k}^{J}\right]
$$

In order to get the solution of the system $(6.2 .48)-(6.2 .49)$ to first order in momentum we expand the fields in the (dimensionless) quantity $p=\frac{k}{4 \pi T}$, as in 5.2.27)-5.2.28, namely

$$
\begin{array}{r}
h_{t}^{\alpha}(u)=h_{t}^{(0), \alpha}(u)+p h_{t}^{(1), \alpha}(u) \\
B_{\alpha}(u)=B_{\alpha}^{(0)}(u)+p B_{\alpha}^{(1)}(u)
\end{array}
$$

The system can be solved perturbatively . To calculate the retarded correlators at $r=\Lambda$ (or, equivalently, at $u=u_{c} \equiv r_{H}^{2} / \Lambda^{2}$ ) we only need to solve the equations for the perturbations with infalling boundary conditions, on the one hand, and boundary conditions $\Phi_{k}^{I}\left(u_{c}\right)=\phi_{k}^{I}$ on the other [69]. This procedure should give us the desired Green's functions, after taking the variation of 12.4 .63 with respect to the fields at $u=u_{c}$ (which act as sources for their corresponding operators). Recall that, as explained in Section 6.2, the bulk-to-boundary propagator must be normalized at $r=\Lambda$, that is, if we have

$$
\Phi_{k}^{I}(u)=F_{J}^{I}(k, u) \phi_{k}^{J}
$$

then $F_{J}^{I}\left(k, u_{c}\right)=1$. Notice that the relation between the boundary value at $u=u_{c}$ and that at $u=0$ is simply $\phi_{k}^{I\left(u_{c}\right)}=F_{J}^{I}\left(k, u_{c}\right) \phi_{k}^{J}{ }^{(0)}$, so that the solution is preserved by these manipulations, as pointed out by [128] and [132, 136, 137]. The retarded two-point functions, from which we are able to read directly the transport coefficients, then have the form

$$
G_{I J}\left(k, u_{c}\right)=-2 \lim _{u \rightarrow u_{c}}\left(\mathcal{A}_{I M}\left(F_{J}^{M}(k, u)\right)^{\prime}+\mathcal{B}_{I J}\right)
$$

Where the $\mathcal{A}_{I J}$ and $\mathcal{B}_{I J}$ matrices are 69

$$
\mathcal{A}=\frac{r_{H}^{4}}{16 \pi G L^{5}} \operatorname{Diag}\left(-3 a f(u), \frac{1}{u},-3 a f(u), \frac{1}{u}\right)
$$




$$
B_{A d S+\partial}=\frac{r_{H}^{4}}{16 \pi G L^{5}}\left[\begin{array}{cccc}
0 & 3 a & 0 & 0 \\
0 & -\frac{3}{u^{2}} & 0 & 0 \\
0 & 0 & 0 & 3 a \\
0 & 0 & 0 & -\frac{3}{u^{2}}
\end{array}\right]
$$

Using again the the effective chemical potential

$$
\mu(\Lambda)=\mu\left(1-\frac{r_{H}^{2}}{\Lambda^{2}}\right)
$$

the result for the anomalous correlators is

$$
\begin{array}{r}
\left\langle\delta J^{x} \delta J^{z}\right\rangle=\frac{i \mu \kappa k}{2 \pi G}\left(1-\frac{r_{H}^{2}}{\Lambda^{2}}\right)=\frac{-i k \mu(\Lambda)}{4 \pi^{2}} \\
\left\langle\delta J^{x} \delta t_{t}^{z}\right\rangle=\left\langle\delta t_{t}^{x} \delta J^{z}\right\rangle=-\frac{i \kappa \mu^{2} k}{4 \pi G}\left(1-\frac{r_{H}^{2}}{\Lambda^{2}}\right)^{2}=\frac{i k \mu(\Lambda)^{2}}{8 \pi^{2}} \\
\left\langle\delta t_{t}^{x} \delta t_{t}^{z}\right\rangle=\frac{i \kappa \mu^{3} k}{6 \pi G}\left(1-\frac{r_{H}^{2}}{\Lambda^{2}}\right)^{3}=\frac{-i k \mu(\Lambda)^{3}}{12 \pi^{2}}
\end{array}
$$

Since $\lim _{\Lambda \rightarrow \infty} \mu(\Lambda)=\mu$, these correlators coincide essentially with the ones derived in 69$]^{4}$

\subsubsection{Compatibility with the flow equations}

The system of first order differential equations (6.2.59)-(6.2.66) must be compatible with the result 6.3.77)-6.3.79 encountered in the previous section. In order to check that it is so, the dissipative correlators play an important role. In the case $\omega=0$ and to $\mathcal{O}(k)$, they read ${ }^{5}$

$$
\begin{array}{r}
G^{x x}=P^{x t}=G_{\epsilon}^{x x}=0 \\
P_{\epsilon}^{x t}=-\frac{r_{H}^{4}}{8 \pi G L^{5} u} f^{2}(u)\left(\frac{f^{\prime}(u)}{f(u)}-\frac{3}{u}\right)
\end{array}
$$

This solution implies that $G^{x x}$ and $P^{x t}=G_{\epsilon}^{x x}$ are of order $\omega$ or higher, whereas $P_{\epsilon}^{x t}$ contains a part which is of order $\mathcal{O}\left(k^{0}, \omega^{0}\right)$ (contact term). The remaining system, after substituting 6.3.80, 6.3.81 and assuming that all the anomalous correlators are at least of $\mathcal{O}(k)$, turns out to be (up to order $k$ )

\footnotetext{
${ }^{4}$ The minus sign found in 6.3 .78 with respect to the result of 69 is due to the fact that in this reference the correlator that is studied is $\left\langle\delta J^{a} \delta t_{b}^{t}\right\rangle$, that differs from $\left\langle\delta J^{a} \delta t_{t}^{b}\right\rangle$ by a factor of $(b$ represents a spatial index) $g_{t t} g^{b b}=-f(u) \rightarrow-1$ at infinity.

${ }^{5}$ The limit $P_{\epsilon}^{x t}(u=0)$ is not well defined because we have not included the corresponding counterterms in 6.2.50, 6.2.51. The reason is that they do not affect the anomalous correlators.
} 


$$
\begin{array}{r}
G^{\prime x x}=0 \\
G^{\prime x z}=-\frac{r_{H}^{2}}{8 \pi G L^{3}} \bar{\kappa} i k \\
P^{\prime x t}=0 \\
P^{\prime z t}-\mu G^{x z}=0 \\
G_{\epsilon}^{\prime x x}=0 \\
G_{\epsilon}^{\prime x z}=\mu G^{x z} \\
P_{\epsilon}^{\prime x t}=-P_{\epsilon}^{x t}\left(\frac{3}{u}-\frac{f^{\prime}(u)}{f(u)}\right)-\frac{3 r_{H}^{4}}{8 \pi G L^{5} u} f(u)\left(a u-\frac{f(u)}{u^{2}}\right) \\
P_{\epsilon}^{\prime z t}-\mu G_{\epsilon}^{x z}=\mu P^{z t}
\end{array}
$$

Equation 6.3.88) is in agreement with 6.3.81). In the end, the 2-point functions associated with dissipative transport coefficients decouple completely. Regarding the anomalous correlators, the above system of equations can be integrated easily, leading to

$$
\begin{array}{r}
G^{x z}=\frac{r_{H}^{2}}{8 \pi G L^{3}} \bar{\kappa} i k\left(1-u_{c}\right) \\
P^{z t}=G_{\epsilon}^{x z}=-\mu \frac{r_{H}^{2}}{16 \pi G L^{3}} \bar{\kappa} i k\left(1-u_{c}\right)^{2} \\
P_{\epsilon}^{x t}=\mu^{2} \frac{r_{H}^{2}}{24 \pi G L^{3}} \bar{\kappa} i k\left(1-u_{c}\right)^{3}
\end{array}
$$

which is the same as 6.3.77)-6.3.79). The role played by the Chern-Simons term in 6.2.44 is crucial to ensure that $G^{x z}$ presents a flow, for in its absence all the anomalous 2-point functions identically vanish.

\subsection{Gravitational Anomaly}

The study of the effect of the Gravitational Anomaly on the definition of the holographic operators is a non-trivial task, for the term $A \wedge R \wedge R$ has not a well defined Dirichlet problem. This makes, strictly speaking, not possible to define generic operators. In Chapter 5, the problem was circumvented by arguing that any possible contribution vanishes asymptotically. However, now we are interested in the value of the transport coefficients at finite cutoff $\Lambda$, and therefore it is necessary to face this issue.

\subsubsection{The Model}

The four dimensional axial gravitational anomaly is induced holographically by a Chern-Simons term of the form

$$
S_{\mathcal{A} C S}=\frac{\lambda}{16 \pi G} \int d^{5} x \sqrt{-g} \epsilon^{M N P Q R} A_{M} R_{B N P}^{A} R_{A Q R}^{B}
$$

This action contributes to the boundary axial current as expected for a mixed anomaly. The comple action is given by equations (5.1.6)-(5.1.8). Adding $S_{C S K}$ ensures that the anomalous Ward identity for gauge transformations depends only on the intrinsic curvature tensor on the boundary at $r=\Lambda$ as in Chapter 5 . Indeed, the covariant current turns out to be

$$
16 \pi G J^{A}=n_{B}\left[F^{A B}-8 \epsilon^{B A C D E} \lambda K_{C F} D_{D} K_{F}^{E}\right]_{r=\Lambda}
$$


with a purely four dimensional divergence that on shell evaluates to

$$
D_{\mu} J^{\mu}=-\frac{1}{16 \pi G} \epsilon^{o p q r}\left[\frac{\kappa}{3} F_{o p} F_{q r}+\lambda R_{(4) b o p}^{a} R_{(4) a q r}^{b}\right]_{r=\Lambda}
$$

where $\epsilon^{o p q r} \equiv \epsilon^{\text {nopqr }}$ is the four dimensional epsilon tensor.

The aforementioned difficulties with the Dirichlet problem make the definition of the stress tensor be much more involved. The main problem is that the bulk action contains a term proportional to $\dot{K}_{i j}$ and hence the variation of the on-shell action is going to depend on $\delta K_{i j}$. Asymptotically there is a way to define the one point function which assumes that in an AdS spacetime the $r$-derivative corresponds to the dilatation operator. Then, a generic field $\phi=e^{(\Delta-d) r} \phi_{(0)}+$ (subleading terms) fulfills $\delta \dot{\phi}=(\Delta-d) e^{(\Delta-d) r} \delta \phi_{(0)}+\ldots$. This makes possible to express $\delta K_{i j}$ in terms of the induced metric and hence to define the one-point function of the energy-momentum tensor. After tedious computations one can obtain that, asymptotically

$$
\begin{array}{r}
T_{j}^{i}=\frac{\sqrt{-h}}{8 \pi G}\left[K_{j}^{i}-K h_{j}^{i}+4 \lambda \epsilon^{(i k l m}\left(\frac{1}{2} F_{k l} R_{(4) j) m}+\nabla_{n}\left(A_{k} R_{(4) j) l m}^{n}\right)\right)\right]_{r \rightarrow \infty} \\
D_{i} T^{i j}=\left[-J_{m} F^{m j}+A^{j} D_{i} J^{i}\right]_{r \rightarrow \infty}
\end{array}
$$

In particular, 6.4.97) is consistent with what one would expect from a system subjected to an external electromagnetic field. Notice also that all $\lambda$-terms vanish if the boundary is flat.

At finite cutoff the previous analysis is not possible anymore, for the spacetime is not AdS and thus one cannot write $\delta K_{i j}$ in terms of $\delta h_{i j}$. Moreover, it is expected that several terms that vanish at infinity would contribute to the energy-momentum tensor at $r=\Lambda$. It is therefore necessary to consider the full higher-derivative system, in which in particular $K_{i j}$ is regarded as an independent variable, in order to be able to obtain an expression for $\left\langle T^{i j}\right\rangle$. We will show however in the next section that the equations of motion turn out to be second order in the shear sector. This allows to calculate the relevent two point functions of energy-momentum tensor and current. The bulk equations of motion are given by expressions (5.1.16)- 5.1 .17 .

\subsubsection{Contribution of the Gravitational Anomaly}

If we vary $S_{\mathcal{A C S}}$, we are left with a term which spoils the variational problem

$$
\frac{\lambda}{2 \pi G} \int_{\partial} \sqrt{-h} \epsilon^{m l q r} A_{m} D_{r} K_{q}^{v} \delta K_{l v}
$$

If we looked for a suitable counterterm to render the Dirichlet problem well-posed, we would end up finding $S_{C S \mathcal{K}}$. Indeed, this boundary contribution was firstly conceived as an analogue to the Gibbons-Hawking-York term. However, after varying $S_{\mathcal{A C S}}+S_{C S \mathcal{K}}$ one realizes that the result

$$
-\frac{\lambda}{2 \pi G} \int_{\partial} \sqrt{-h} \epsilon^{m l q r} D_{r} A_{m} \delta K_{q}^{v} K_{l v}
$$

is still problematic. Even worse, 6.4.99 can not be canceled easily, for, for instance, the ansatz

$$
\frac{\lambda}{2 \pi G} \int_{\partial} \sqrt{-h} \epsilon^{m l q r} D_{r} A_{m} K_{q}^{v} K_{l v}
$$


is automatically zero. Thus in principle, there is not a straightforward way of having a well defined variational problem for this system.

On the other hand, as aforementioned, we need $S_{C S \mathcal{K}}$ to have a four dimensional anomalous Ward identity at the boundary, so we will keep it. A hypothetical generic counterterm (if it exists) capable of solving all the problems, would probably ruin (6.4.95) and therefore, by physical means, should not be considered. Even though the variational problem is not well-posed, we will still be able to derive the equations of motion by means of the analogue of the Euler-Lagrange equations for higher-derivative theories. The difficulty therefore reduces to the question How to treat 6.4.99) holographically? Note that in [51] it was implicitly assumed that the Dirichlet problem is correctly defined, so we should go a little bit further in this case. Specializing for the shear sector, which is the one that interests us, and at second order in perturbations, (6.4.99) reads

$$
-\frac{\lambda}{2 \pi G} \int_{\partial} \sqrt{-h} \epsilon^{m l q r} D_{r} \delta A_{m} \delta K_{q}^{v} K_{l v}
$$

Other possible terms would vanish in the background (6.2.45). The strategy would be the following: Since 6.4.101) does not affect two point functions involving only energymomentum tensors or only currents, we know how to compute the correlators $\left\langle T_{t}^{x} T_{t}^{z}\right\rangle$ and $\left\langle J^{x} J^{z}\right\rangle$. 6.4.101) only plays a role when calculating $\left\langle T_{t}^{x} J^{z}\right\rangle,\left\langle J^{x} T_{t}^{z}\right\rangle$, and hence those are the ones for which the discussion of [51] does not apply.

Following the method detailed in Section 6.3, it turns out that, taking only into account the gravitational anomaly

$$
\begin{aligned}
\left\langle J^{x} J^{z}\right\rangle & =0 \\
\left\langle T_{t}^{x} T_{t}^{z}\right\rangle & =-i k \frac{\mu\left(1-u_{c}\right) T^{2}}{12}
\end{aligned}
$$

(note that we have directly substituted the value of $\lambda$ for a single left-handed fermion $\left.\lambda / G=-\frac{1}{48 \pi}\right)$. The above results point again towards an effective chemical potential of the form $\mu\left(1-u_{c}\right)$. Therefore, on physical grounds, we expect the appearance of an effective temperature also. Note that the flows of the effective quantities must be consistent in the sense that they must be the same, no matter what correlator we are focusing on. Equations 6.4.102-6.4.103 hint at the existence of an effective temperature for the system; this temperature does not flow with the cutoff scale, being always identical to the Hawking temperature. This conclusion is in agreement with the asymptotic values obtained before. So we resolve that 6.4 .99 must be treated in such a way that $\left\langle T_{t}^{x} J^{z}\right\rangle,\left\langle J^{x} T_{t}^{z}\right\rangle$, at finite cutoff, are consistent with a non-flowing temperature.

It turns out that the method to achieve it is precisely the one that one would anticipate by general considerations: Taking advantage of the fact that the equations of motion for the shear sector

$$
\begin{array}{r}
0=h_{t}^{\alpha^{\prime \prime}}(u)-\frac{h_{t}^{\alpha^{\prime}}(u)}{u}-3 a u B_{\alpha}^{\prime}(u)+i \bar{\lambda} k \epsilon_{\alpha \beta}\left[\left(24 a u^{3}-6(1-f(u))\right) \frac{B_{\beta}(u)}{u}\right. \\
\left.+\left(9 a u^{3}-6(1-f(u))\right) B_{\beta}^{\prime}(u)+2 u\left(u h_{t}^{\beta^{\prime}}(u)\right)^{\prime}\right], \\
0=\quad B_{\alpha}^{\prime \prime}(u)+\frac{f^{\prime}(u)}{f(u)} B_{\alpha}^{\prime}(u)-\frac{h_{t}^{\alpha^{\prime}}(u)}{f(u)} \\
+i k \epsilon_{\alpha \beta}\left(\frac{3}{u f(u)} \bar{\lambda}\left(\frac{2}{a}(f(u)-1)+3 u^{3}\right) h_{t}^{\beta^{\prime}}(u)+\bar{\kappa} \frac{B_{\beta}(u)}{f(u)}\right),
\end{array}
$$


happen to be of second order in derivatives (where $\bar{\lambda}, \bar{\kappa}$ and $a$ are prescribed by (5.2.24)), we can solve completely the evolution as we did in Section 3.3 (imposing in-falling B.C. at the Horizon and Dirichlet B.C. at the boundary). Once the solutions are known (see Appendix 7.3), 6.4.99) will in general give a well determined surface contribution (when evaluated on-shell) that must be taken into account to calculate $\left\langle T_{t}^{x} J^{z}\right\rangle,\left\langle J^{x} T_{t}^{z}\right\rangle$. The result so obtained presents no flow in the temperature part.

To be more concise, the boundary term $(6.4 .99)$ to be considered has the following form

$$
-\frac{i k \lambda r_{H}^{2} \epsilon_{\alpha \beta}}{2 \pi G L^{4}} \int_{\partial} u f^{\prime}(u) a_{\beta}(k) h_{t}^{\prime \alpha}(-k)
$$

whose contribution, up to first order in $k$, is summarized

$$
-\frac{i k \lambda r_{H}^{2} \epsilon_{\alpha \beta}}{2 \pi G L^{4}} \int_{\partial} u \frac{f^{\prime 2}(u)}{f\left(u_{c}\right)} a_{\beta}^{(0)}(k) \tilde{H}_{\beta}^{(0)}(-k)
$$

(Notice the factor $\sim \frac{1}{f(\Lambda)}$ introduced to normalize the perturbation (Appendix 7.3)). So the effect of $(6.4 .99)$ on the Green's functions can be reformulated as a modification, prescribed by 6.4.107), of the $\mathcal{B}_{I J}$ matrix.

Even though (6.4.107) only affects the correlator $\left\langle T_{t}^{\alpha} J^{\beta}\right\rangle, S_{\mathcal{A C S}}+S_{\mathcal{C S K}}$ induces automatically a non-vanishing value for the components $\mathcal{A}_{14}=\mathcal{A}_{32}^{*}$ of the matrix $\mathcal{A}$. These contributions, which are perfectly treatable within the framework of [51], give rise to a correction of $\left\langle J^{\alpha} T_{t}^{\beta}\right\rangle$ which is precisely of the same form of the one implemented by 6.4.107). As will be mentioned below, this turns out to be sufficient for the consistency condition (7.0.1) to hold.

The final form of the matrices $\mathcal{A}_{I J}$ and $\mathcal{B}_{I J}$ after implementing the shift driven by the Gravitational Anomaly is given by

$$
\begin{aligned}
& \mathcal{A}=\frac{r_{H}^{4}}{16 \pi G L^{5}}\left[\begin{array}{cccc}
-3 a f(u) & 0 & 0 & -\frac{4 i \lambda k L}{r_{H}^{2}} u f^{\prime}(u) \\
0 & \frac{1}{u} & 0 & \frac{i 8 \lambda k L \mu}{r_{H}^{2}} u \\
0 & \frac{4 i \lambda k L}{r_{H}^{2}} u f^{\prime}(u) & -3 a f(u) & 0 \\
0 & -\frac{i 8 \lambda k L \mu}{r_{H}^{2}} u & 0 & \frac{1}{u}
\end{array}\right] \\
& B_{A d S+\partial}=\frac{r_{H}^{4}}{16 \pi G L^{5}}\left[\begin{array}{cccc}
0 & 3 a & 0 & 0 \\
0 & -\frac{3}{u^{2}} & 4 i \lambda k L \frac{9 a u^{3}-6(1-f(u))}{u r_{H}^{2}} & 0 \\
0 & 0 & 0 & 3 a \\
-4 i \lambda k L \frac{9 a u^{3}-6(1-f(u))}{u r_{H}^{2}} & 0 & 0 & -\frac{3}{u^{2}}
\end{array}\right] \\
& B_{\partial C S}=\frac{r_{H}^{4}}{16 \pi G L^{5}}\left[\begin{array}{cccc}
0 & 0 & 0 & 0 \\
0 & 0 & -\frac{4 i \lambda k L}{r_{H}^{2}} u \frac{f^{\prime}(u)^{2}}{f\left(u_{c}\right)} & 0 \\
0 & 0 & 0 & 0 \\
\frac{4 i \lambda k L}{r_{H}^{2}} u \frac{f^{\prime}(u)^{2}}{f\left(u_{c}\right)} & 0 & 0 & 0
\end{array}\right]
\end{aligned}
$$


The resulting anomalous 2-point functions are

$$
\begin{aligned}
\left\langle J^{x} J^{z}\right\rangle & =\frac{i k \kappa \mu\left(1-u_{c}\right)}{2 G \pi}=-i k \frac{\mu(\Lambda)}{4 \pi^{2}} \\
\left\langle J^{x} T_{t}^{z}\right\rangle & =-\frac{i k \kappa\left(1-u_{c}\right)^{2} \mu^{2}}{4 G \pi}-\frac{i k \lambda(-2+a)^{2} r_{H}^{2}}{2 G L^{4} \pi}=i k\left(\frac{\mu^{2}\left(1-u_{c}\right)^{2}}{8 \pi^{2}}+\frac{T^{2}}{24}\right) \\
\left\langle T_{t}^{x} J^{z}\right\rangle & =-\frac{i k \kappa\left(1-u_{c}\right)^{2} \mu^{2}}{4 G \pi}-\frac{i k \lambda(-2+a)^{2} r_{H}^{2}}{2 G L^{4} \pi}=i k\left(\frac{\mu^{2}\left(1-u_{c}\right)^{2}}{8 \pi^{2}}+\frac{T^{2}}{24}\right) \\
\left\langle T_{t}^{x} T_{t}^{z}\right\rangle & =\frac{i k \kappa\left(1-u_{c}\right)^{3} \mu^{3}}{6 G \pi}+\left(1-u_{c}\right) \mu \frac{i k \lambda(-2+a)^{2} r_{H}^{2}}{G L^{4} \pi}=-i k\left(\frac{\mu^{3}\left(1-u_{c}\right)^{3}}{12 \pi^{2}}+\frac{\mu(\Lambda) T^{2}}{12}\right)
\end{aligned}
$$

Observe that it is straightforward to verify that equations (14.0.3)- 14.0.6 are compatible with the asymptotic value computed in Section 5 . Notice also that the temperature part remains constant as we move the boundary. The flow of the different correlators is consistent with respect to each other and the hypothesis of an effective chemical potential $\mu(\Lambda)=\mu\left(1-\frac{r_{H}^{2}}{\Lambda^{2}}\right) \equiv \mu\left(1-u_{c}\right)$ is reinforced by the results extracted from the terms proportional to $\lambda$. 


\section{Chapter 7}

\section{Discussion and Conclusion}

In Section 5.1 we have defined a holographic bottom up model that implements the mixed gauge-gravitational anomaly in four dimensional field theory via a mixed gaugegravitational Chern-Simons term. We have discussed its holographic renormalization and have shown that the Chern-Simons term does not introduce new divergencies. As a first application of this theory we have computed the anomalous magnetic and vortical conductivities from a charged black hole background and have found the $T^{2}$ terms characteristic for the contribution of the gravitational anomaly.

The most important result is certainly that the numerical values of the conductivities coincide precisely with the ones obtained at weak coupling in [70] (see also the Introduction, Section 3.3.1).

We have studied a holographic system with only one anomalous $U(1)$ symmetry. It should however present no problem to generalize our calculation to the case with additional non-abelian symmetries and various types of mixed anomalies, e.g. mimicking the usual interplay of axial and vector symmetries where gauge Bardeen counterterms are necessary to implement the correct anomaly structures in the currents 134, 135.

In addition, Chapter 6 is devoted to the study of the holographic cutoff flow of the anomalous transport coefficients. This has been done by using the model that implements both the axial and the mixed axial-gravitational anomalies. The flow has been studied by analyzing the dependence of the anomalous Green's functions on the radial position, $\Lambda$, of the boundary. We have presented several prescriptions to compute such flow and finally obtained it by adapting the method implemented in Chapter 5.1 for the case $\Lambda \rightarrow \infty$. It is a remarkable fact that the chiral magnetic conductivity suffers from a flow even in the non-backreacted case. In fact, this could have been anticipated by noticing that regularity at the horizon imposes that in the deep IR the constitutive relations are only compatible with an electric conductivity ( 128$]$ ), so that if a system exhibits a chiral magnetic conductivity in the UV it must be due to a non-trivial flow. When considering the gravitational anomaly, a Dirichlet boundary condition is not enough anymore to define the variational problem properly. A generic definition of suitable operators, if any, therefore requires further discussion in this case. Here we have simply focused on computing 2-point functions, without discussing general definitions of the corresponding operators. The term which spoils the variational principle has been dealt with by considering its effect on the on-shell action. This procedure, which can be seen to be the most natural one by using physical arguments, yields 2-point functions that are consistent and whose flows do not get in contradiction with the result found in the absence of gravitational anomaly. Moreover, 
in the spirit of [51], that the matrix of correlators $\mathcal{G}_{I J}$ obeys

$$
\frac{d}{d u}\left(\mathcal{G}-\mathcal{G}^{\dagger}\right)=0
$$

represents a non-trivial consistency check. The result (14.0.3)-(14.0.6 shows that the temperature remains constant (Hawking temperature) whereas the chemical potential presents a flow that is easily interpretable in terms of the energy necessary to bring a unit of charge from the horizon to the boundary. Observe, however, that all the correlators are written for a metric with $g_{t t} \sim-r^{2} f(r)$, and hence there is an implicit redshift factor between observers living in one hypersurface placed at $r=\Lambda$ and another one at $r=\Lambda^{\prime}$.

From the point of view of the boundary theory, these outcomes indicate that the pure gauge Chern-Simons term does not affect the boundary operators but influences the anomalous correlators through the flow equations, forcing them to have a non-vanishing value at the boundary, whereas the gravitational-gauge Chern-Simons term happens not to have any impact by means of the evolution equations, but to induce new covariant contributions, that are first order in $k$, to the operators, so that the constant $T^{2}$ part is present at any value of the $r$-coordinate. 


\section{Appendix of Part II}

\subsection{Holographic Renormalization}

Holographic Renormalization is a technique that allows us, given a gravitional theory, to define the holographic dictionary and to obtain finite correlators, i.e. extract the divergences of the on-shell action.

\subsubsection{Technical details on Holographic Renormalization}

The renormalization procedure follows from an expansion of the four dimensional quantities in eigenfunctions of the dilatation operator

$$
\delta_{D}=2 \int d^{4} x \gamma_{i j} \frac{\delta}{\delta \gamma_{i j}} .
$$

This expansion reads

$$
\begin{aligned}
K_{j}^{i} & =K_{(0)}{ }_{j}^{i}+K_{(2)}{ }_{j}^{i}+K_{(4)}{ }_{j}^{i}+\tilde{K}_{(4)}{ }_{j}^{i} \log e^{-2 r}+\cdots, \\
A_{i} & =A_{(0) i}+A_{(2) i}+\tilde{A}_{(2) i} \log e^{-2 r}+\cdots,
\end{aligned}
$$

where

$$
\begin{aligned}
& \delta_{D} K_{(0)}{ }_{j}^{i}=0, \quad \delta_{D} K_{(2)}{ }_{j}^{i}=-2 K_{(2)}{ }_{j}^{i}, \\
& \delta_{D} K_{(4) j}^{i}=-4 K_{(4) j}^{i}-2 \tilde{K}_{(4) j}^{i}, \quad \delta_{D} \tilde{K}_{(4) j}{ }_{j}^{i}=-4 \tilde{K}_{(4) j}{ }_{j}^{i}, \\
& \delta_{D} A_{(0) i}=0, \quad \delta_{D} A_{(2) i}=-2 A_{(2) i}-2 \tilde{A}_{(2) i}, \\
& \delta_{D} \tilde{A}_{(2) i}=-2 \tilde{A}_{(2) i} \text {. }
\end{aligned}
$$

Given the above expansion of the fields one has to solve the equations of motion in its Codazzi form, order by order in a recursive way. To do so one needs to identify the leading order in dilatation eigenvalues at which each term contributes. One has

$$
\begin{aligned}
& \gamma_{i j} \sim \mathcal{O}(-2), \quad \gamma^{i j} \sim \mathcal{O}(2), \quad E_{i} \sim \mathcal{O}(2), \quad \hat{F}_{i j} \sim \mathcal{O}(0), \\
& \sqrt{-\gamma} \sim \mathcal{O}(-4), \quad K_{j}^{i} \sim \mathcal{O}(0), \quad \hat{R}^{i}{ }_{j k l} \sim \mathcal{O}(0), \quad \nabla_{i} \sim \mathcal{O}(0) .
\end{aligned}
$$

Note that for convenience of notation we define $\mathcal{O}(n)$ if the leading eigenvalue of the dilatation operator is $-n$. In practice, in the renormalization procedure one needs to use the equations of motion Eqs. 7.2.54 and 7.2.55 up to $\mathcal{O}(2)$ and $\mathcal{O}(4)+\mathcal{O}(\tilde{4})$ respectively. Up to $\mathcal{O}(0)$ they write

$$
\begin{aligned}
& 0=K_{(0)}^{2}-K_{(0)}^{i} K_{(0)}{ }_{i}^{j}-2 \Lambda, \\
& 0=\dot{K}_{(0){ }_{j}^{i}}+K_{(0)} K_{(0)}{ }_{j}^{i}-\frac{2 \Lambda}{(d-1)} \delta_{j}^{i} .
\end{aligned}
$$


Order $\mathcal{O}(2)$ writes

$$
\begin{aligned}
& 0=2 K_{(0)} K_{(2)}-2 K_{(0)}{ }_{j}^{i} K_{(2) i}-\hat{R}, \\
& 0=\left.\dot{K}_{j}^{i}\right|_{(2)}+K_{(0)} K_{(2)}{ }_{j}^{i}+K_{(2)} K_{(0)}{ }_{j}^{i}-\hat{R}_{j}^{i},
\end{aligned}
$$

and finally orders $\mathcal{O}(4)$ and $\mathcal{O}(\tilde{4})$ for Eq. 7.2 .55 write respectively

$$
\begin{aligned}
& 0=2 K_{(0)} K_{(4)}+K_{(2)}^{2}-2 K_{(0) j}^{i} K_{(4) i}^{j}-K_{(2) j}^{i} K_{(2) i}^{j}+\frac{1}{4} \hat{F}_{(0) i j} \hat{F}_{(0)}{ }^{i j}, \\
& 0=2\left(K_{(0)} \tilde{K}_{(4)}-K_{(0)}^{i} \tilde{K}_{(4)}^{j}\right) \log e^{-2 r} .
\end{aligned}
$$

The derivative on $r$ can be computed by using

$$
\frac{d}{d r}=\int d^{4} x \dot{\gamma}_{k m} \frac{\delta}{\delta \gamma_{k m}}=2 \int d^{4} x K_{m}^{l} \gamma_{l k} \frac{\delta}{\delta \gamma_{k m}} .
$$

By inserting in this equation the expansion of $K_{j}^{i}$ given by Eq. (7.1.2), one gets $d / d r \simeq \delta_{D}$ at the lowest order. Taking into account this, the computation of $K_{(0)}{ }_{j}^{i}$ is trivial if one considers the definition of $K_{i j}$, i.e.

$$
K_{(0) i j}=\left.\frac{1}{2} \dot{\gamma}_{i j}\right|_{(0)}=\frac{1}{2} \delta_{D} \gamma_{i j}=\gamma_{i j} .
$$

Then the result up to $\mathcal{O}(0)$ is

$$
K_{(0)}^{i}{ }_{j}^{i}=\delta_{j}^{i}, \quad K_{(0)}=d .
$$

Inserting this result into Eq. (7.1.6) or 7.1.7) one arrives at the well known cosmological constant

$$
\Lambda=\frac{d(d-1)}{2} .
$$

We have used in Eq. 7.1.7 that $\dot{K}_{(0)}^{i}{ }_{i}^{i}=\delta_{D} K_{(0)}{ }_{j}^{i}=0$. The result for $K_{(2)}$ follows immediately from Eqs. (7.1.8) and 7.1.14,

$$
K_{(2)}:=P=\frac{\hat{R}}{2(d-1)} .
$$

In order to proceed with the computation of $K_{(2)}{ }_{j}^{i}$ from Eq. 7.1 .9 , we should evaluate first $\left.\dot{K}_{j}^{i}\right|_{(2)}$. Using the definition of $d / d r$ given by Eq. 7.1.12, it writes

$$
\begin{aligned}
\left.\dot{K}_{j}^{i}\right|_{(2)} & =2 \int d^{4} x K_{(0)}{ }_{m}^{l} \gamma_{l k} \frac{\delta}{\delta \gamma_{k m}} K_{(2)} \stackrel{i}{j}+2 \int d^{4} x K_{(2)}{ }_{m}^{l} \gamma_{l k} \frac{\delta}{\delta \gamma_{k m}} K_{(0)}{ }_{j}^{i} \\
& =2 \int d^{4} x \gamma_{k m} \frac{\delta}{\delta \gamma_{k m}} K_{(2)} \stackrel{i}{j}=\delta_{D} K_{(2)}^{i} \underset{j}{i}=-2 K_{(2)} \underset{j}{i} .
\end{aligned}
$$

Because $K_{(0)}{ }_{j}^{i}$ is the Kronecker's delta, the second term after the first equality is zero, while the first one becomes the dilatation operator acting over $K_{(2)}{ }_{j}^{i}$. Then one gets from Eq. (7.1.9) the result

$$
K_{(2)}{ }_{j}^{i}:=P_{j}^{i}=\frac{1}{(d-2)}\left[\hat{R}_{j}^{i}-P \delta_{j}^{i}\right]
$$


Note that the trace of $K_{(2)}{ }_{j}^{i}$ agrees with Eq. 7.1.16. Using all the results above it is straightforward to solve for orders $\mathcal{O}(4)$ and $\mathcal{O}(4)$. From Eqs. 7.1.10 and 7.1.11 one gets respectively

$$
\begin{aligned}
K_{(4)} & =\frac{1}{2(d-1)}\left[P_{j}^{i} P_{i}^{j}-P^{2}-\frac{1}{4} \hat{F}_{(0) i j} \hat{F}_{(0)}{ }^{i j}\right] \\
\tilde{K}_{(4)} & =0 .
\end{aligned}
$$

In order to compute the counterterm for the on-shell action, besides the equations of motion an additional equation is needed. Following Ref. [21], one can introduce a covariant variable $\theta$ and write the on-shell action as

$$
S_{\text {on-shell }}=\frac{1}{8 \pi G} \int_{\partial} d^{4} x \sqrt{-\gamma}(K-\theta) .
$$

Then computing $\dot{S}_{\text {on-shell }}$ from Eq. 7.1.21, and comparing it with the result obtained by using Eqs. (7.1.47)-7.1.49), one gets the following equation

$$
\begin{aligned}
0= & \dot{\theta}+K \theta-\frac{1}{(d-1)}\left(2 \Lambda+\frac{1}{2} E_{i} E^{i}+\frac{1}{4} \hat{F}_{i j} \hat{F}^{i j}\right)-\frac{2}{3} \kappa \epsilon^{i j k l} A_{i} E_{j} \hat{F}_{k l} \\
& -\frac{12 \lambda}{(d-1)} \epsilon^{i j k l}\left[A_{i} \hat{R}^{n}{ }_{m k l} D_{n} K_{j}^{m}+E_{i} K_{j m} D_{k} K_{l}^{m}+\frac{1}{2} \hat{F}_{i k} K_{j m} \dot{K}_{l}^{m}\right] .
\end{aligned}
$$

The variable $\theta$ admits also an expansion in eigenfunctions of $\delta_{D}$ of the form

$$
\theta=\theta_{(0)}+\theta_{(2)}+\theta_{(4)}+\tilde{\theta}_{(4)} \log e^{-2 r}+\cdots,
$$

where

$$
\begin{aligned}
& \delta_{D} \theta_{(0)}=0, \quad \delta_{D} \theta_{(2)}=-2 \theta_{(2)}, \\
& \delta_{D} \theta_{(4)}=-4 \theta_{(4)}-2 \tilde{\theta}_{(4)}, \quad \delta_{D} \tilde{\theta}_{(4)}=-4 \tilde{\theta}_{(4)} .
\end{aligned}
$$

Inserting expansion 7.1.23 into Eq. 7.1.22, one gets the following identities

$$
\begin{aligned}
& 0=\dot{\theta}_{(0)}+K_{(0)} \theta_{(0)}-\frac{2 \Lambda}{(d-1)}, \\
& 0=\left.\dot{\theta}\right|_{(2)}+K_{(2)} \theta_{(0)}+K_{(0)} \theta_{(2)} \\
& 0=\left.\dot{\theta}\right|_{(4)}+K_{(4)} \theta_{(0)}+K_{(2)} \theta_{(2)}+K_{(0)} \theta_{(4)}-\frac{1}{4(d-1)} \hat{F}_{(0) i j} \hat{F}_{(0)}{ }^{i j} \\
& 0=\left.\dot{\theta}\right|_{(\tilde{4})}+\left(\theta_{(0)} \tilde{K}_{(4)}+K_{(0)} \tilde{\theta}_{(4)}\right) \log e^{-2 r}
\end{aligned}
$$

corresponding to orders $\mathcal{O}(0), \mathcal{O}(2), \mathcal{O}(4)$ and $\mathcal{O}(\tilde{4})$ respectively. Following the same procedure as shown in Eqs. 77.1.13 and 7.1.17, one gets

$$
\dot{\theta}_{(0)}=0, \quad \dot{\theta}_{(2)}=\delta_{D} \theta_{(2)}=-2 \theta_{(2)} .
$$

At this point one can solve Eqs. 7.1.25 and 7.1.26 to get

$$
\theta_{(0)}=1, \quad \theta_{(2)}=\frac{P}{(2-d)} .
$$


Higher orders are a little bit more involved. Using the definition of $d / d r$, then $\left.\dot{\theta}\right|_{(4)}$ writes

$$
\begin{aligned}
\left.\dot{\theta}\right|_{(4)} & =2 \int d^{4} x K_{(0)}{ }_{m}^{l} \gamma_{l k} \frac{\delta}{\delta \gamma_{k m}} \theta_{(4)}+2 \int d^{4} x K_{(4)}{ }_{m}^{l} \gamma_{l k} \frac{\delta}{\delta \gamma_{k m}} \theta_{(0)}+2 \int d^{4} x K_{(2)}{ }_{m}^{l} \gamma_{l k} \frac{\delta}{\delta \gamma_{k m}} \theta_{(2)} \\
& =\delta_{D} \theta_{(4)}+\frac{2}{(2-d)} \int d^{4} x P_{k m} \frac{\delta}{\delta \gamma_{k m}} P .
\end{aligned}
$$

Note that the second term after the first equality vanishes, while the first one writes in terms of $\delta_{D}$. To evaluate the last term at the r.h.s. of eq. (7.1.31) we use

$$
\delta \hat{R}=-\hat{R}^{k m} \delta \gamma_{k m}+D^{k} D^{m} \delta \gamma_{k m}-\gamma^{k m} D_{l} D^{l} \delta \gamma_{k m} .
$$

After a straightforward computation, one gets

$$
\left.\dot{\theta}\right|_{(4)}=-4 \theta_{(4)}-2 \tilde{\theta}_{(4)}+\frac{1}{(d-1)(d-2)}\left[(d-2) P_{j}^{i} P_{i}^{j}+P^{2}+D_{i}\left(D^{i} P-D^{j} P_{j}^{i}\right)\right] .
$$

Inserting Eq. 7.1.33 into Eq. 77.1.27) one can solve the latter, and the result is ${ }^{1}$

$$
\tilde{\theta}_{(4)}=\frac{1}{4}\left[P_{j}^{i} P_{i}^{j}-P^{2}-\frac{1}{4} \hat{F}_{(0) i j} \hat{F}_{(0)}^{i j}+\frac{1}{3} D_{i}\left(D^{i} P-D^{j} P_{j}^{i}\right)\right] .
$$

The computation of $\left.\dot{\theta}\right|_{(\tilde{4})}$ follows in a similar way, and one gets $\left.\dot{\theta}\right|_{(\tilde{4})}=-4 \tilde{\theta}_{(4)} \log e^{-2 r}$. By inserting it into Eq. (7.1.28), this equation is trivially fulfilled.

The counterterm of the action can be read out from Eq. (7.1.21) by using $K$ and $\theta$ computed up to order $\mathcal{O}(\tilde{4})$, i.e.

$S_{c t}=-S_{\text {on-shell }}=-\frac{1}{8 \pi G} \int_{\partial} d^{4} x \sqrt{-\gamma}\left[\left(K_{(0)}-\theta_{(0)}\right)+\left(K_{(2)}-\theta_{(2)}\right)+\left(\tilde{K}_{(4)}-\tilde{\theta}_{(4)}\right) \log e^{-2 r}\right]$.

From this equation and Eqs. (7.1.14), (7.1.16), (7.1.20), 7.1.30) and (7.1.34), one finally gets

$$
\begin{aligned}
S_{c t}=-\frac{(d-1)}{8 \pi G} & \int_{\partial} d^{4} x \sqrt{-\gamma}\left[1+\frac{1}{(d-2)} P\right. \\
& \left.-\frac{1}{4(d-1)}\left(P_{j}^{i} P_{i}^{j}-P^{2}-\frac{1}{4} \hat{F}_{(0) i j} \hat{F}_{(0)}{ }^{i j}\right) \log e^{-2 r}\right] .
\end{aligned}
$$

The last term in Eq. (7.1.34 is a total derivative, and so it doesn't contribute to the action. As a remarkable fact we find that there is no contribution in the counterterm coming from the gauge-gravitational Chern-Simons term. This is because this term only contributes at higher orders. Indeed as explained above, in the renormalization procedure we use Eqs. 7.2.55 and 7.1.22 up to orders $\mathcal{O}(0), \mathcal{O}(2), \mathcal{O}(4)$ and $\mathcal{O}(\tilde{4})$, and Eq. 7.2.54 up to orders $\mathcal{O}(0)$ and $\mathcal{O}(2)$. We have explicitly checked that the $\lambda$ dependence starts contributing at $\mathcal{O}(6)$ in all these three equations. ${ }^{2}$ This means that the gauge-gravitational Chern-Simons term does not induce new divergences, and so the renormalization is not modified by it.

\footnotetext{
${ }^{1}$ This result for $\tilde{\theta}_{(4)}$ includes a total derivative term which has not been computed in Ref. 21. To compute $\tilde{\theta}_{(4)}$, in this reference the authors derive the elegant relation $\tilde{\theta}_{(4)}=\frac{(d-1)}{2} K_{(4)}+\tilde{K}_{(4)}$. This identity is however valid modulo total derivative terms.

${ }^{2}$ Note that $\dot{K}_{j}^{i}$ and $\dot{\theta}$ induce terms proportional to $\lambda$. Up to order $\mathcal{O}(4)+\mathcal{O}(\tilde{4})$ these operators write $\left.\dot{K}_{j}^{i}\right|_{(4)+(\tilde{4})}=-4 K_{(4)}^{i}+\ldots$, and $\left.\dot{\theta}\right|_{(4)+(\tilde{4})}=-4 \theta_{(4)}+\ldots$, where the dots indicate extra terms which are $\lambda$-independent. The only $\lambda$-dependence could appear in $K_{(4)}^{i} j$ and $\theta_{(4)}$, but these contributions are precisely cancelled by other terms in Eqs. 7.2.54 and 7.1.22 respectively, so that these equations become $\lambda$-dependent only at $\mathcal{O}(6)$ and higher.
} 


\subsubsection{Holographic Renormalization of the Mixed Anomaly}

In this section, we aim to show that the term in the action that implements the Mixed gauge-gravitational anomaly does not induce new divergences. In order to go through the steps of the holographic renormalization program within the Hamiltonian approach [21, 138], first of all we establish some notations. Without loss of generality we choose a gauge with vanishing shift vector $N_{A}=0$, lapse $N=1$ and $A_{r}=0$. So we can use four dimensional (boundary) indices and denote them by small latin letters. We therefore also write $\epsilon(t x y z)=+1$ and $\epsilon_{i j k l}=\sqrt{-h} \epsilon(i j k l)$. In this gauge the bulk metric can be written as

$$
d s^{2}=d r^{2}+\gamma_{i j} d x^{i} d x^{j} .
$$

The non vanishing Christoffel symbols are

$$
\begin{aligned}
-\Gamma_{i j}^{r} & =K_{i j}=\frac{1}{2} \dot{\gamma}_{i j}, \\
\Gamma_{j r}^{i} & =K_{j}^{i},
\end{aligned}
$$

and $\hat{\Gamma}_{j k}^{i}$ are four dimensional Christoffel symbols computed with $\gamma_{i j}$. Dot denotes differentiation respect $r$. All other components of the extrinsic curvature vanish, i.e. $K_{r r}=$ $K_{r i}=0$. Another useful table of formulas is

$$
\begin{aligned}
\dot{\hat{\Gamma}}_{k i}^{l} & =D_{k} K_{i}^{l}+D_{i} K_{k}^{l}-D^{l} K_{k i}, \\
R^{r}{ }_{i r j} & =-\dot{K}_{i j}+K_{i l} K_{j}^{l}, \\
R^{k}{ }_{r j r} & =-\dot{K}_{j}^{k}-K_{l}^{k} K_{j}^{l}, \\
R^{r}{ }_{i j k} & =D_{k} K_{i j}-D_{j} K_{i k}, \\
R^{l}{ }_{k r i} & =D_{k} K_{i}^{l}-D^{l} K_{i k}, \\
R^{i}{ }_{j k l} & =\hat{R}^{i}{ }_{j k l}-K_{k}^{i} K_{j l}+K_{l}^{i} K_{j k} .
\end{aligned}
$$

Note that indices are now raised and lowered with $\gamma_{i j}$, e.g. $K=\gamma^{i j} K_{i j}$, and intrinsic four dimensional curvature quantities are denoted with a hat, so $\hat{R}^{i}{ }_{j k l}$ is the intrinsic four dimensional Riemann tensor on the $r=$ const surface. Finally the Ricci scalar is

$$
R=\hat{R}-2 \dot{K}-K^{2}-K_{i j} K^{i j} .
$$

Now we can calculate the off shell action. It is useful to divide it up in three terms. The first one is the usual gravitational bulk and gauge terms with the usual Gibbons-Hawking term. After some computations we get

$$
\begin{aligned}
S^{0} & =\frac{1}{16 \pi G} \int d^{5} x \sqrt{-\gamma}\left[\hat{R}+2 \Lambda+K^{2}-K_{i j} K^{i j}-\frac{1}{2} E_{i} E^{i}-\frac{1}{4} \hat{F}_{i j} \hat{F}^{i j}\right] \\
S_{C S}^{1} & =-\frac{\kappa}{12 \pi G} \int d^{5} x \sqrt{-\gamma} \epsilon^{i j k l} A_{i} E_{j} \hat{F}_{k l} \\
S_{C S}^{2} & =-\frac{8 \lambda}{16 \pi G} \int d^{5} x \sqrt{-\gamma} \epsilon^{i j k l}\left[A_{i} \hat{R}^{n}{ }_{m k l} D_{n} K_{j}^{m}+E_{i} K_{j m} D_{k} K_{l}^{m}+\frac{1}{2} \hat{F}_{i k} K_{j m} \dot{K}_{l}^{m}\right]
\end{aligned}
$$

We have used implicitly here the gauge $A_{r}=0$ and denoted $\dot{A}_{i}=E_{i}$. The purely four dimensional field strength is denoted with a hat.

Of particular concern is the last term in $S_{C S}^{2}$ which contains explicitly the normal derivative of the extrinsic curvature $\dot{K}_{i j}$. For this reason the field equations will be generically of third order in $r$-derivatives and that means that we can not define a well-posed Dirichlet problem by fixing the $\gamma_{i j}$ and $K_{i j}$ alone but generically we would need to fix also 
$\dot{K}_{i j}$. Having applications to holography in mind we can however impose the boundary condition that the metric has an asymptotically AdS expansion of the form

$$
\gamma_{i j}=e^{2 r}\left(g_{i j}^{(0)}+e^{-2 r} g_{i j}^{(2)}+e^{-4 r}\left(g_{i j}^{(4)}+2 r \tilde{g}_{i j}^{(4)}\right)+\cdots\right) .
$$

Using the on-shell expansion of $K_{i j}$ obtained before we can show that the last term in the action does not contribute in the limit $r \rightarrow \infty$. Therefore the boundary action depends only on the boundary metric $\gamma_{i j}$ but not on the derivative $\dot{\gamma}_{i j}$. This is important because otherwise the dual theory would have additional operators that are sourced by the derivative. Similar issues have arisen before in the holographic theory of purely gravitational anomalies of two dimensional field theories 139 141]. Alternatively one could restrict the field space to configurations with vanishing gauge field strength on the boundary. Then the last term in $S_{C S}^{2}$ is absent. We note that the simple form of the higher derivative terms arises only if we include $S_{C S K}$ in the action. An analogous term in four dimensional Chern-Simons gravity has been considered before in 142 .

The result one gets for the counterterm coming from the regularization of the boundary action is

$$
\begin{aligned}
S_{c t}=-\frac{(d-1)}{8 \pi G} & \int_{\partial} d^{4} x \sqrt{-\gamma}\left[1+\frac{1}{(d-2)} P\right. \\
& \left.-\frac{1}{4(d-1)}\left(P_{j}^{i} P_{i}^{j}-P^{2}-\frac{1}{4} \hat{F}_{(0) i j} \hat{F}_{(0)}{ }^{i j}\right) \log e^{-2 r}\right],
\end{aligned}
$$

where

$$
P=\frac{\hat{R}}{2(d-1)}, \quad P_{j}^{i}=\frac{1}{(d-2)}\left[\hat{R}_{j}^{i}-P \delta_{j}^{i}\right] .
$$

As a remarkable fact there is no contribution in the counterterm coming from the gaugegravitational Chern-Simons term. This has also been derived in 143 in a similar model that does however not contain $S_{C S K}$.

\subsection{Codazzi form of Equations of Motion}

We project the equations of motion (5.1.16) and (5.1.17) into the boundary surface and the orthogonal direction and rewrite them in terms of quantities at the regulated boundary. Doing so we get a set of two dynamical equations

$$
\begin{aligned}
0= & \dot{E}^{i}+K E^{i}+D_{j} \hat{F}^{j i}-4 \epsilon^{i j k l}\left(\kappa E_{j} \hat{F}_{k l}+4 \lambda \dot{K}_{j}^{s} D_{l} K_{s k}+2 \lambda \hat{R}_{t k l}^{s} D_{s} K_{j}^{t}\right. \\
& \left.+4 \lambda K_{k s} K_{l}^{t} D_{t} K_{j}^{s}+4 \lambda K_{s t} K_{j}^{t} D_{l} K_{k}^{s}(\rangle \cdot 2.53\right) \\
0= & \dot{K}_{j}^{i}+K K_{j}^{i}-\hat{R}_{j}^{i}+\frac{1}{2} E^{i} E_{j}+\frac{1}{2} \hat{F}^{i m} \hat{F}_{j m}-\frac{\delta_{j}^{i}}{(d-1)}\left(2 \Lambda+\frac{1}{2} E^{m} E_{m}+\frac{1}{4} \hat{F}^{l m} \hat{F}_{l m}\right) \\
+ & 2 \lambda\left[-2 \epsilon^{(i k l m} \partial_{r}\left(\hat{F}_{k l} \dot{K}_{m j}\right)+2 \epsilon^{i i k l m} \partial_{r}\left(\hat{F}_{k l} K_{m s} K_{j]}^{s}\right)+2 \epsilon^{i k l m} \hat{F}_{k l} K_{j s}\left(\dot{K}_{m}^{s}+K_{t}^{s} K_{m}^{t}\right)\right. \\
& \left.-\epsilon^{k l m n} \hat{F}_{k l}\left(K_{s}^{(i} \hat{R}_{j) m n}^{s}+2 K_{m}^{(i} \dot{K}_{n j}\right)-2 K_{s}^{i} K_{m}^{s} K_{n j}\right)+4 \epsilon^{(i k l m} \partial_{r}\left(E_{k} D_{m} K_{j) l}\right) \\
& +2 \epsilon^{(i k l m} D_{s}\left(\hat{F}_{k l}\left(D_{j)} K_{m}^{s}-D^{s} K_{j) m}\right)\right)+4 \epsilon^{i k l m} E_{k} K_{j s} D_{l} K_{m}^{s} \\
& \left.-4 \epsilon^{k l m n} E_{k} K_{l}^{(i} D_{n} K_{m j)}+2 \epsilon^{(i k l m} D_{s}\left(E_{k}\left(\hat{R}^{s}{ }_{j) l m}-2 K_{l}^{s} K_{j) m}\right)\right)\right],
\end{aligned}
$$


and three constraints

$$
\begin{aligned}
0= & K^{2}-K_{i j} K^{i j}-\hat{R}-2 \Lambda-\frac{1}{2} E_{i} E^{i}+\frac{1}{4} \hat{F}_{i j} \hat{F}^{i j} \\
& +8 \lambda \epsilon^{i j k l}\left(D_{m}\left(\hat{F}_{i j} D_{k} K_{l}^{m}\right)+\hat{F}_{i j} K_{k m} \dot{K}_{l}^{m}+2 E_{i} K_{j t} D_{l} K_{k}^{t}\right), \\
0= & D_{j} K^{j i}-D^{i} K+\frac{1}{2} E_{j} \hat{F}^{j i}+2 \lambda \epsilon^{k l m i} D_{j}\left[2 E_{k} D_{l} K_{m}^{j}+\hat{F}_{k l}\left(\dot{K}_{m}^{j}+K_{s}^{j} K_{m}^{s}\right)\right] \\
& +\lambda \epsilon^{k l m n}\left\{2 \hat{F}_{k l} K_{j}^{i} D_{m} K_{n}^{j}+D_{j}\left[F_{k l}\left(\hat{R}^{i j}{ }_{n m}+2 K_{n}^{i} K_{m}^{j}\right)\right]\right. \\
& \left.+2 E_{k} K_{m}^{j} \hat{R}^{i}{ }_{j n l}+2 \hat{F}_{k l} K_{m}^{j}\left(D^{i} K_{n j}-D_{j} K_{n}^{i}\right)+2 \partial_{r}\left(\hat{F}_{k l} D_{n} K_{m}^{i}\right)\right\} \\
0 & \left.D_{i} E^{i}-\epsilon^{i j k l}\left(\kappa \hat{F}_{i j} \hat{F}_{k l}+\lambda \hat{R}^{s}{ }_{t i j} \hat{R}^{t}{ }_{s k l}+4 \lambda K_{i s} K_{j}^{t} \hat{R}_{t k l}^{s}+8 \lambda D_{i} K_{s j} D_{l} K_{k}^{s}\right)\right\rangle
\end{aligned}
$$

with the notation

$$
X^{(i}{ }_{j)}:=\frac{1}{2}\left(X^{i}{ }_{j}+X_{j}{ }^{i}\right), \quad X^{[i}{ }_{j]}:=\frac{1}{2}\left(X^{i}{ }_{j}-X_{j}{ }^{i}\right) .
$$

We take Eq. (7.2.58) as a definition, and it should be applied also when $X$ includes derivatives on $r$, for instance $X^{(i} \dot{K}_{l j)}=\frac{1}{2}\left(X^{i} \dot{K}_{l j}+X_{j} \dot{K}_{l}^{i}\right)$.

\subsection{Solutions at zero frequency and normalized at finite cut- off $u_{c}$}

\subsubsection{Case $\lambda=0$}

$$
\begin{array}{r}
B^{\alpha}(u)=\bar{B}^{\alpha}+\bar{H}^{\alpha}\left(u-u_{c}\right)-\frac{i \bar{\kappa} k \epsilon_{\alpha \beta}}{2(1+4 a)^{2}\left(-1+u_{c}\left(-1+a u_{c}\right)\right)} \times \\
\times\left(( 1 + 4 a ) ( u - u _ { c } ) \left(\bar{H}^{\beta}+\bar{H}^{\beta} u_{c}+a\left(3 \bar{B}^{\beta}\left(2+u_{c}\right)+\bar{H}^{\beta}\left(4-u_{c}\left(2+3 u_{c}\right)\right)\right)+\right.\right. \\
+2 \sqrt{1+4 a}(-2+a(-2+3 u))\left(\bar{B}^{\beta}-\bar{H}^{\beta} u_{c}\right)\left(-1+u_{c}\left(-1+a u_{c}\right)\right)\left(\operatorname{ArcTanh}\left[\frac{-1+2 a u}{\sqrt{1+4 a}}\right]+\right. \\
\left.\left.+\operatorname{ArcTanh}\left[\frac{1-2 a u_{c}}{\sqrt{1+4 a}}\right]\right)\right)
\end{array}
$$




$$
\begin{array}{r}
H_{t}^{\alpha}(u)=-\frac{1}{2(-1-4 a)^{3 / 2}\left(-1+u_{c}\right)\left(-1+u_{c}\left(-1+a u_{c}\right)\right)^{2}}(-1+u) \times \\
\times\left(-2(-1-4 a)^{3 / 2} \bar{H}^{\alpha}(-1+u(-1+a u))\left(-1+u_{c}\left(-1+a u_{c}\right)\right)+\right. \\
+k \bar{\kappa} \epsilon_{\alpha \beta}\left(-i(\sqrt{-1-4 a}-i \sqrt{1+4 a}) \bar{H}^{\beta}(1+u)\left(1+u_{c}\right)+\right. \\
+a^{2} 3 \bar{B}^{\beta}\left(2 i \sqrt{-1-4 a} u_{c}^{2}+i \sqrt{-1-4 a} u u_{c}^{2}+\sqrt{1+4 a} u^{2}\left(2+u_{c}\right)\right)+ \\
+a^{2} \bar{H}^{\beta}\left(2 i \sqrt{-1-4 a}\left(2-3 u_{c}\right) u_{c}^{2}+i \sqrt{-1-4 a} u\left(4-3 u_{c}\right) u_{c}^{2}+\sqrt{1+4 a} u^{2}\left(4-u_{c}\left(2+3 u_{c}\right)\right)\right)- \\
-3 i a \bar{B}^{\beta}\left(2 \sqrt{-1-4 a}-2 i \sqrt{1+4 a}+2 \sqrt{-1-4 a} u_{c}-i \sqrt{1+4 a} u_{c}\right)- \\
-3 i a u \bar{B}^{\beta}\left(\sqrt{-1-4 a}-2 i \sqrt{1+4 a}+\sqrt{-1-4 a} u_{c}-i \sqrt{1+4 a} u_{c}\right)+ \\
+a \bar{H}^{\beta}\left(-4 i \sqrt{-1-4 a}-4 \sqrt{1+4 a}+\sqrt{1+4 a} u^{2}\left(1+u_{c}\right)\right)+ \\
+a \bar{H}^{\beta} u_{c}\left(2 i \sqrt{-1-4 a}+2 \sqrt{1+4 a}+7 i \sqrt{-1-4 a} u_{c}+3 \sqrt{1+4 a} u_{c}\right)+ \\
+u a \bar{H}^{\beta} u_{c}(-i \sqrt{-1-4 a}-4 \sqrt{1+4 a})+ \\
+6 i a k(-1+u(-1+a u)) \bar{\kappa} \epsilon_{\alpha \beta}\left(\bar{B}^{\beta}-\bar{H}^{\beta} u_{c}\right)\left(-1+u_{c}\left(-1+a u_{c}\right)\right) \operatorname{ArcTan}\left[\frac{-1+2 a u}{\sqrt{-1-4 a}}\right]+ \\
\left.+6 a k(-1+u(-1+a u)) \bar{\kappa} \epsilon_{\alpha \beta}\left(\bar{B}^{\beta}-\bar{H}^{\beta} u_{c}\right)\left(-1+u_{c}\left(-1+a u_{c}\right)\right) \operatorname{ArcTanh}\left[\frac{1-2 a u_{c}}{\sqrt{1+4 a}}\right]\right)
\end{array}
$$

\subsubsection{Case $\kappa=0$}

$$
\begin{array}{r}
B^{\alpha}(u)=\bar{B}^{\alpha}+\bar{H}^{\alpha}\left(u-u_{c}\right)+ \\
+\frac{1}{6(2-a) a^{3}}(-2+a) k \bar{u}_{c} \epsilon_{\alpha \beta}\left(\frac{2 i(-2+a(-2+3 u)) \operatorname{ArcTanh}\left[\frac{1-2 a u}{\sqrt{1+4 a}}\right]}{(1+4 a)^{3 / 2}} \times\right. \\
\times\left(4 \bar{H}^{\beta}+a\left(3(1+a(7+2 a(7+a))) \bar{B}^{\beta}+4(8+a(2+a)(9+2 a)) \bar{H}^{\beta}-\right.\right. \\
\left.\left.-3(1+a(7+2 a(7+a))) \bar{H}^{\beta} u_{c}\right)\right)+ \\
-3 a^{2}\left(3 \bar{B}^{\beta}(-4+u)\left(1+u_{c}\right)+\bar{H}^{\beta}\left(u(10+3 u)+u(10+3 u) u_{c}-2(-8+u) u_{c}^{2}-4\left(7+6 u_{c}\right)\right)\right)+ \\
+8 \bar{H}^{\beta}\left(1+u_{c}\right)+ \\
+a^{4}\left(\bar{H}^{\beta}\left(12+u_{c}\left(18+(-59+12 u(2+3 u)) u_{c}\right)\right)+3 \bar{B}^{\beta}\left(2+u_{c}\left(5+12\left(-1+u-u_{c}\right) u_{c}\right)\right)\right)+ \\
+a^{3} 9 \bar{B}^{\beta}\left(4+u\left(-4+\left(-4+u_{c}\right) u_{c}\right)-u_{c}\left(-5+u_{c}+u_{c}^{2}\right)\right)+ \\
\left.+a^{3} \bar{H}^{\beta}\left(29+\left(23-72 u_{c}\right) u_{c}+9 u^{2}\left(-4+\left(-4+u_{c}\right) u_{c}\right)+6 u\left(-4+u_{c}\left(-4+5 u_{c}\right)\right)\right)\right)+ \\
+(-2+a(-2+3 u))\left(-1+u_{c}\left(-1+a u_{c}\right)\right)\left(2 \left(4 \bar{H}^{\beta}+a\left(3(1+a(7+2 a(7+a))) \bar{B}^{\beta}+\right.\right.\right. \\
\left.\left.+4(8+a(2+a)(9+2 a)) \bar{H}^{\beta}-3(1+a(7+2 a(7+a))) \bar{H}^{\beta} u_{c}\right)\right) \operatorname{ArcTanh}\left[\frac{1-2 a u_{c}}{\sqrt{1+4 a}}\right]+ \\
+(1+a)(1+4 a)^{3 / 2}\left(-4 \bar{H}^{\beta}+a\left(-3 \bar{B}^{\beta}-4 \bar{H}^{\beta}+3 \bar{H}^{\beta} u_{c}\right)\right) \times \\
\left.\left.\left.\times\left(\log [-1+u(-1+a u)]-\log \left[-1+u_{c}\left(-1+a u_{c}\right)\right]\right)\right)\right)\right)
\end{array}
$$


where $\frac{a}{b} \equiv \frac{1}{(-1-4 a)^{3 / 2}\left(1+u_{c}-a u_{c}^{2}\right)}$

$$
\begin{array}{r}
H_{t}^{\alpha}(u)=\bar{H}^{\alpha} \frac{(-1+u)(-1+u(-1+a u))}{\left(-1+u_{c}\right)\left(-1+u_{c}\left(-1+a u_{c}\right)\right)}+\frac{(1-u) \epsilon_{\alpha \beta}}{2(-1-4 a)^{3 / 2} a^{2}} k\left(1+u-a u^{2}\right) \bar{u}_{c} \times \\
\times\left(-\frac{1}{(-1+u(-1+a u))\left(-1+u_{c}\left(-1+a u_{c}\right)\right)} 2 a \sqrt{1+4 a}\left(u-u_{c}\right) \times\right. \\
\times\left(4 \bar{H}^{\beta}(1+u)\left(1+u_{c}\right)+a \bar{B}^{\beta}\left(3+5 u+5(1+u) u_{c}\right)+\right. \\
+a \bar{H}^{\beta}\left(18+u(25+u)+22 u_{c}+u(25+u) u_{c}-4(1+u) u_{c}^{2}\right)- \\
-3 a^{2} \bar{B}^{\beta}\left(-5+2 u^{2}\left(1+u_{c}\right)+u_{c}\left(-7+2 u_{c}\right)+u\left(-7+2\left(-3+u_{c}\right) u_{c}\right)\right)+ \\
\bar{H}^{\beta} a^{2}\left(18+24 u_{c}-22 u_{c}^{2}-6 u^{3}\left(1+u_{c}\right)+u\left(39+5\left(7-5 u_{c}\right) u_{c}\right)-u^{2}\left(1+u_{c}+u_{c}^{2}\right)\right)+ \\
+3 a^{3} \bar{B}^{\beta}\left(1-8 u_{c}^{2}-u u_{c}\left(3+8 u_{c}\right)+2 u^{2}\left(-4+\left(-4+u_{c}\right) u_{c}\right)\right)+ \\
+\bar{H}^{\beta} a^{3}\left(4-4 u_{c}\left(2+5 u_{c}\right)+u^{2}\left(-20+\left(-20+u_{c}\right) u_{c}\right)+6 u^{3}\left(-4+\left(-4+u_{c}\right) u_{c}\right)\right)- \\
+\bar{H}^{\beta} u\left(5+7 u_{c}\left(1+5 u_{c}\right)\right)+\bar{H}^{\beta} a^{4}\left(4 u+4 u_{c}+6 u u_{c}+(-2+u(-5+4 u(5+6 u))) u_{c}^{2}\right)+ \\
+2\left(4 \bar{H}^{\beta}+a\left(3(1+a(7+2 a(7+a))) \bar{B}^{\beta}+4(8+a(2+a)(9+2 a)) \bar{H}^{\beta}-\right.\right. \\
\left.\left.-3(1+a(7+2 a(7+a))) \bar{H}^{\beta} u_{c}\right)\right)\left(\operatorname{ArcTanh}\left[\frac{-1+2 a u}{\sqrt{1+4 a}}\right]+\operatorname{ArcTanh}\left[\frac{1-2 a u_{c}}{\sqrt{1+4 a}}\right]\right)+ \\
+(1+a)(1+4 a)^{3 / 2}\left(-4 \bar{H}^{\beta}+a\left(-3 \bar{B}^{\beta}+\bar{H}^{\beta}\left(-4+3 u_{c}\right)\right)\right) \times \\
\left.\times\left(\log [-1+u(-1+a u)]-\log \left[-1+u_{c}\left(-1+a u_{c}\right)\right]\right)\right)
\end{array}
$$

\subsection{Equations of motion for the shear sector}

These are the complete linearized set of six dynamical equations of motion

$$
\begin{aligned}
0= & B_{\alpha}^{\prime \prime}(u)+\frac{f^{\prime}(u)}{f(u)} B_{\alpha}^{\prime}(u)+\frac{b^{2}}{u f(u)^{2}}\left(w^{2}-f(u) k^{2}\right) B_{\alpha}(u)-\frac{h_{t}^{\alpha^{\prime}}(u)}{f(u)} \\
& +i k \epsilon_{\alpha \beta}\left(\frac{3}{u f(u)} \bar{\lambda}\left(\frac{2}{3 a}(f(u)-1)+u^{3}\right) h_{t}^{\beta^{\prime}}(u)+\bar{\kappa} \frac{B_{\beta}(u)}{f(u)}\right) \\
0= & h_{t}^{\alpha^{\prime \prime}}(u)-\frac{h_{t}^{\alpha^{\prime}}(u)}{u}-\frac{b^{2}}{u f(u)}\left(k^{2} h_{t}^{\alpha}(u)+h_{y}^{\alpha}(u) w k\right)-3 a u B_{\alpha}^{\prime}(u) \\
& i \bar{\lambda} k \epsilon_{\alpha \beta}\left[\left(24 a u^{3}-6(1-f(u)) \frac{B_{\beta}(u)}{u}+\left(9 a u^{3}-6(1-f(u))\right) B_{\beta}^{\prime}(u)\right.\right. \\
& \left.+2 u\left(u h_{t}^{\beta^{\prime}}(u)\right)^{\prime}-\frac{2 u b^{2}}{f(u)}\left(h_{y}^{\beta}(u) w k+h_{t}^{\beta}(u) k^{2}\right)\right] \\
0 & h_{y}^{\alpha^{\prime \prime}}(u)+\frac{(f / u)^{\prime}}{f / u} h_{y}^{\alpha^{\prime}}(u)+\frac{b^{2}}{u f(u)^{2}}\left(w^{2} h_{y}^{\alpha}(u)+w k h_{t}^{\alpha}(u)\right)+2 u i k \bar{\lambda} \epsilon_{\alpha \beta}\left[u h_{y}^{\beta^{\prime \prime}}(u)\right. \\
& \left.+\left(9 f(u)-6+3 a u^{3}\right) \frac{h_{y}^{\beta^{\prime}}(u)}{f(u)}+\frac{b^{2}}{f(u)^{2}}\left(w k h_{t}^{\beta}(u)+w^{2} h_{y}^{\beta}(u)\right)\right]
\end{aligned}
$$

and two constraints for the fluctuations at $w, k \neq 0$

$$
\begin{aligned}
0= & w\left(h_{t}^{\alpha^{\prime}}(u)-3 a u B_{\alpha}(u)\right)+f(u) k h_{y}^{\alpha^{\prime}}(u)+i k \bar{\lambda} \epsilon_{\alpha \beta}\left[2 u^{2}\left(w h_{t}^{\beta^{\prime}}+f(u) k h_{y}^{\beta^{\prime}}(u)\right)\right. \\
& \left.+\left(9 a u^{3}-6(1-f(u))\right) B_{\beta}(u)\right] .
\end{aligned}
$$




\section{Part III}

\section{Exotic Holographic Superfluids}


As a brief introduction, we include here some notions of s-wave Holographic superfluids, as they were firstly constructed in 3,144$]^{3}$ (for a review, see 148,149$]$ ). Typically, in holography what we aim to obtain is a non-sourced scalar operator developing a VEV. The first ingredient that we need is a chemical potential; the fact that the dual theory is conformal implies that the temperature can be fixed to be the identity and therefore the temperature only cannot work as a tunable parameter. Hence, our parameter will be $\bar{\mu} \equiv \mu / T$. According to the AdS/CFT dictionary, this forces us to consider a gauge field in the bulk $A_{\mu}$, which in this introduction we take to be associated to a $U(1)$ symmetry, for simplicity. A minimal suitable ansatz seems then to be simply

$$
S=\frac{1}{2 \kappa^{2}} \int d^{4} x \sqrt{-g}\left(R-2 \Lambda-\frac{1}{4} F_{\mu \nu} F^{\mu \nu}-\overline{D_{\mu} \Psi} D^{\mu} \Psi-V(|\Psi|)\right)
$$

where $R$ is the Ricci scalar, $F_{\mu \nu}$ is the $U(1)$ field-strenght and from now on we assume $\Lambda=-\frac{3}{L^{2}}$. The field $\Psi$ is a complex scalar, i.e. $D_{\mu}=\partial_{\mu}-i g A_{\mu}$. Moreover, we will use the most simple potential $V(|\Psi|)=-2|\Psi|^{2} / L^{2}$ (corresponding to a mass $m^{2}=-2 / L^{2}$ ). Thus the above bottom-up model does not contain any quartic term in the scalar field. The reason is that the theory (7.4.1) suffices to trigger a unsourced VEV for the scalar. The mechanism is the following. In the absence of scalar field ( $\Psi=0$ everywhere) there is RN-BB solution to the equations of motion that we derive from 7.4.1, which is associated to a thermal state at finite chemical potential in the dual field theory. The near horizon geometry (which corresponds to the IR of the theory) of the RN-BB is known to be $A d S_{2} \times S^{2}$ [REF.]. In order to break spontaneously the U(1) symmetry, we will make the IR unstable under scalar perturbations. The way to accomplish this is by making the scalar field to acquire a mass whose value lies below the BF bound of $A d S_{2}$ (IR geometry), but strictly above of the BF bound of $A d S_{4}$, so that the $U V$ is stable. Now, the term proportional to $A_{\mu} A^{\mu}|\Psi|^{2}$ acts as an effective negative mass for the scalar field, so a large enough source $A_{t}(r \rightarrow \infty) \sim \mu$ could be enough to trigger a non-trivial VEV for the scalar field. This is indeed what occurs, at sufficiently high chemical potential the charged scalar field develops an expectation value and triggers a symmetry breaking phase transition towards a superfluid phase.

After the VEV is formed, the Higgs mechanism provides an effective mass for the gauge field in the bulk of the form $\sim A_{\mu} A^{\mu}\left\langle|\Psi|^{2}\right\rangle$, consistent with gauge invariance, which in turn gives rise to the presence of a London current and the Meissner effect in the dual field theory, after weakly gauging the symmetry [3]. Hence, we conclude that the $U(1)$ global symmetry of the boundary CFT gets broken spontaneously.

Let us remark that henceforth we will be working in the so called probe approximation or probe limit. One could get to that decoupling limit by rescaling the gauge field and scalar with respect to the gauge couling $g$, namely $A_{\mu} \rightarrow A_{\mu} / g$ and $\Psi \rightarrow \Psi / g$. This rescaling implies the Maxwell-scalar sector in 7.4.1 to acquire an overall $g^{-2}$ factor. Sending now $g \rightarrow \infty$, we can decouple such a sector from the gravity sector, leading to

$$
S_{\text {probe }}=\frac{1}{2 \kappa^{2} g^{2}} \int d^{4} x \sqrt{-g}\left(-\frac{1}{4} F_{\mu \nu} F^{\mu \nu}-\overline{D_{\mu} \Psi} D^{\mu} \Psi-V(|\Psi|)\right) .
$$

This is the action we will be working with in subsequent chapters. Notice that in the probe limit the metric is fixed and all the dynamics is provided by the gauge field and the

\footnotetext{
${ }^{3}$ There exist also p-wave and even d-wave holographic superfluids constructed in AdS/CFT (see for instance 145 147])
} 
scalar.

The study of the QNMs for the s-wave $U(1)$ superfluid was first carried out in [2]. Since the basic physics of superfluids is the one of spontaneous symmetry breaking it can be expected that known results such as the existence of a Goldstone boson carry over to the holographic models.

In the next Section we will generalize 7.4 .2 for it to enjoy a $U(2)$ symmetry that we will break spontaneously; we get our inspiration from the (zero-temperature) model introduced in Section 4.4.1. We will see that a subsector of our $U(2)$ model is, up to linear level, identical to the $U(1)$ holographic superconductor, described by (7.4.2). Therefore, we will review also the results on the QNMs and conductivities of the $U(1)$ model.

According to the usual holographic dictionary a local bulk symmetry corresponds to a global symmetry in the boundary conformal field theory. We would therefore most naturally be led to a model in which we gauge the global $U(2)$ symmetry of 4.4 .21 ) and put it into an AdS Schwarzschild background. In order to trigger spontaneous symmetry breaking we introduce a chemical potential via a boundary value for the temporal component of the overall, Abelian $U(1)$ gauge field. This is then our gauged model.

Alternatively we might ask what are the minimal ingredients necessary to trigger spontaneous symmetry breaking. The chemical potential resides entirely in the overall $U(1)$ factor. The other three $S U(2)$ gauge fields are not needed to achieve symmetry breaking. Therefore we can choose as a sort of minimal setup a model in which the $S U(2)$ symmetry stays global in the bulk of AdS. This is a somewhat unusual realization of the symmetry from the boundary conformal field theory point of view. There are no conserved currents associated to this $S U(2)$ symmetry, nevertheless all states and operators fall naturally into representations of this symmetry group since it is a global symmetry of the bulk and it is also not broken by any of the boundary conditions. This setup constitutes our ungauged model and we will study it in detail in the next section.

Let us note here one more technical detail: the field theoretic model of this section is most naturally viewed as living in four space time dimensions. In the following our holographic models will be dual to field theories living in three space time dimensions in order to stay as close as possible to the well-studied holographic $U(1)$ s-wave superfluid of 2, 144]. This is however of no relevance to the essential features of the models, i.e. the existence and the nature of the hydrodynamic and Goldstone modes. In Chapter 9 we will analyze the stability issues at finite superfluid velocity. Our results will be interpreted in the framework of the possible existence of a Landau criterion at strong coupling in holography. Notice tha this criterion would imply the existence of a limiting velocity for type I NG bosons, that we will call $v_{c}$. For type II NG bosons, we expect $v_{c}$ to vanish. Chapter 10 is devoted to the construction of a new phase in which an s-wave and p-wave condensates coexist. 


\section{Chapter 8}

\section{Non-superconducting BECs and Type-II Nambu-Goldstone Bosons}

In this section we generalize the results on the QNM spectrum to models with $U(2)$ symmetry, by means of two different models. In the first one we simply add a second scalar field of the same mass, we will call this the ungauged model. A second model also includes gauge fields for the whole $U(2)$ symmetry. The difference between the two models is as follows. In the ungauged model only the $U(1)$ symmetry is local in the bulk. It has however a global $S U(2)$ symmetry ${ }^{1}$ under which the scalar fields transform as a doublet. According to the holographic dictionary this model contains only one conserved current, corresponding to the single gauge field in the bulk. The dual field theory inherits of course the global $S U(2)$ symmetry of the bulk but this symmetry is not generated by operators in the dual conformal field theory. This is similar to the decoupling limit in which we are working and in which the fluctuations of the metric are suppressed. The dual field theory has then strictly speaking no energy momentum tensor. In usual four dimensional Lagrangian field theories Noether's theorem guarantees that we can always construct a conserved charge generating a given symmetry of the Lagrangian. In holographically defined field theories the existence of a four dimensional Lagrangian is a priori not guaranteed and therefore Noether's theorem does not straightforwardly apply. This is the case here. Although the dual field theory has the $S U(2)$ symmetry (and Poincaré covariance) it does not contain operators generating these symmetries. We can speak of these symmetries as an outer automorphism of the operator algebra of the dual field theory ${ }^{2}$. Physically the difference between the two models is that the ungauged one is a one-component fluid (there is only one notion of charge) whereas the gauged one is a two component fluid. In the latter case the charges are the expectation values of the zero-component of the currents in the Cartan subalgebra of the $U(2)$ symmetry.

Although this ungauged model does not contain conserved currents for the $S U(2)$ symmetry and therefore many of the standard proofs about existence of Goldstone bosons do not strictly apply we find a new ungapped mode in the QNM spectrum of the scalars. This mode is however not a standard Goldstone boson with linear dispersion relation but

\footnotetext{
${ }^{1}$ Although global symmetries are not expected in a consistent theory of quantum gravity they can be obtained in certain decompactification limits of string theory: e.g. by wrapping branes on cycles and then taking the volume of the cycle to infinity so that the effective gauge coupling on the branes goes to zero leaving only a global symmetry on them.

${ }^{2} \mathrm{~A}$ string theory example for such a situation is the theory based on the small $\mathcal{N}=4$ superconformal algebra on the world sheet. This algebra possesses a large $S O(4)=S U(2) \times S U(2)$ symmetry acting on the four supercharges of which only one $S U(2)$ is represented through chiral currents on the worldsheet.
} 
a so-called type II Goldstone mode whose energy depends quadratically on momentum.

The second model we consider has a scalar field doublet coupled to the full set of $U(2)$ gauge fields. We switch on a chemical potential only for the overall $U(1)$ symmetry. Therefore the high temperature phase has the full $U(2)$ symmetry. At low temperatures this symmetry is broken to $U(1)$. In this model the dual field theory contains currents for all the $U(2)$ symmetries. We can therefore also study the conductivities.

We shall now consider the symmetry breaking pattern of the boundary theory dual to the gauged holographic model. We point out that through the theorems presented in Section 4.4 the presence of a type II Goldstone boson in the spectrum is guaranteed. As in the model studied in Section 4.4.1, there are in total four symmetry generators. The symmetry is broken from $U(2)$ to $U(1)$ and so there are three broken generators. In the broken phase the charges corresponding to the overall $U(1)$ and the Cartan $U(1)$ generator inside $S U(2)$ receive vacuum expectation values. Therefore the rank of the matrix $B$ (equation (4.4.20) is two and so the number of type I and type II Goldstone bosons should add up to two. This is precisely what we will find in the QNM spectrum, one ungapped mode with linear dispersion relation and one ungapped mode with quadratic dispersion relation.

We also note that the ungauged model satisfies Goldstone's theorem and the counting rule of Chadha and Nielsen 4.4.19). It violates however the more refined counting rule 4.4.20. In a strict sense this model only has one symmetry generator since it has only one $U(1)$ gauge field in the bulk. Therefore the counting rule 4.4.20 would suggest the existence of only one Goldstone boson, the number of broken generators is one and the matrix $B$ vanishes trivially.

\subsection{The ungauged model}

We will now study the holographic model where the condensation of a charged scalar breaks a global $S U(2)$ symmetry in the bulk. We shall look at the spectrum of quasinormal modes on both sides of the phase transition and study their dispersion relations. Since the simple $U(1)$ s-wave holographic superfluid constitutes a subsector of this as well as of the gauged model we will also use the opportunity to briefly review the most salient features of its QNM spectrum.

The minimal holographic model containing a type II Goldstone boson consists of a scalar doublet of $S U(2)$ charged under a $U(1)$ gauge field. The Lagrangian is given by

$$
\mathcal{L}=\left(-\frac{1}{4} F^{\mu \nu} F_{\mu \nu}-m^{2} \Psi^{\dagger} \Psi-\left(D^{\mu} \Psi\right)^{\dagger} D_{\mu} \Psi\right)
$$

where

$$
\Psi=\left(\begin{array}{c}
\lambda \\
\psi
\end{array}\right), \quad D_{\mu}=\partial_{\mu}-i A_{\mu}
$$

and $A_{\mu}$ is the Abelian gauge field. The mass of the scalar field is chosen to be $m^{2}=-2 / L^{2}$. This is basically the same as the model in 144 except that we have added a second scalar field $\lambda$ with the same mass. Because of the degeneracy in the mass the model possesses in addition to the bulk-local $U(1)$ symmetry a bulk-global $S U(2)$ symmetry. Note that the global $S U(2)$ symmetry is a priori not enough to set the field $\lambda(r)=0$. But we are interested in un-sourced static solutions for the scalar fields, i.e. we assume that the leading non-normalizable mode is not switched on. The solution space is then a two dimensional complex vector space spanned by the vevs of the operators dual to the scalar 
fields. On this parameter space we can act with the global $S U(2)$ symmetry to set the operator corresponding to the field $\lambda$ equal to zero. Since now the non-normalizable and the normalizable mode of $\lambda$ are set to zero it follows that $\lambda(r)=0$.

We will be working in the probe limit, in which the coupling of the gauge field is very large and the backreaction of the matter fields onto the metric can be neglected. The background metric is then taken to be the Schwarzschild-AdS black brane

$$
\begin{aligned}
& d s^{2}=-f(r) d t^{2}+\frac{d r^{2}}{f(r)}+\frac{r^{2}}{L^{2}}\left(d x^{2}+d y^{2}\right), \\
& f(r)=\frac{r^{2}}{L^{2}}-\frac{M}{r} .
\end{aligned}
$$

The horizon of the black hole is located at $r_{H}=M^{1 / 3} L^{2 / 3}$ and its Hawking temperature is $T=\frac{3 r_{H}}{4 \pi L^{2}}$. In the following we use dimensionless coordinates

$$
(r, t, x, y) \rightarrow\left(r_{H} \rho, \frac{L^{2}}{r_{H}} \bar{t}, \frac{L^{2}}{r_{H}} \bar{x}, \frac{L^{2}}{r_{H}} \bar{y}\right) .
$$

These rescalings allow us to set $M=r_{H}=1$ in the dimensionless system. In order to switch on a finite chemical potential in the boundary theory, the bulk Maxwell field

$$
A=\chi(\rho) d \bar{t},
$$

must take a non-zero value at the boundary. The equations of motion for the background fields are

$$
\begin{aligned}
& \chi^{\prime \prime}+\frac{2}{\rho} \chi^{\prime}-\frac{2 \psi^{2}}{f} \chi=0, \\
& \psi^{\prime \prime}+\left(\frac{f^{\prime}}{f}+\frac{2}{\rho}\right) \psi^{\prime}+\frac{\chi^{2}}{f^{2}} \psi-\frac{m^{2}}{f} \psi=0 .
\end{aligned}
$$

Notice that the system above is precisely the original $U(1)$ holographic superconductor first studied in [144]. To ensure finiteness of the norm of the current at the horizon, we have to demand the scalar field to be regular whereas the gauge field has to vanish $\chi(\rho=1)=0$. With these boundary conditions, the asymptotic behavior of the fields at the conformal boundary is

$$
\begin{gathered}
\chi=\bar{\mu}-\frac{\bar{n}}{\rho}+O\left(\frac{1}{\rho^{2}}\right), \\
\psi=\frac{\psi_{1}}{\rho}+\frac{\psi_{2}}{\rho^{2}}+O\left(\frac{1}{\rho^{3}}\right) .
\end{gathered}
$$

For the chosen value of the scalar mass, both terms in the scalar asymptotics correspond to normalizable modes [150]. Considering one or the other as the vacuum expectation value of a dual boundary operator leads to two different theories. In what follows we will consider only the case in which $\psi_{1}$ is interpreted as the coupling and $\psi_{2}$ as the vev of a mass dimension two operator.

The dimensionless parameters are related with the physical quantities by

$$
\begin{aligned}
\bar{\mu} & =\frac{3}{4 \pi T} \mu, \\
\bar{n} & =\frac{9}{16 \pi^{2} T^{2} L^{2}} n, \\
\psi_{1} & =\frac{3}{4 \pi T L^{2}} J_{\mathcal{O}}, \\
\psi_{2} & =\frac{9}{16 \pi^{2} T^{2} L^{4}}\langle\mathcal{O}\rangle,
\end{aligned}
$$


where $\mu, n$ and $J_{\mathcal{O}},\langle\mathcal{O}\rangle$ are the chemical potential, charge density and source and expectation value of an operator $\mathcal{O}$ of dimension 2, respectively. From now on we set $L=1$. In the following we will work in the grand canonical ensemble. In practice we vary the dimensionless parameter $\bar{\mu}$. Because of the underlying conformal symmetry this can then be thought of as either fixing the chemical potential $\mu$ and varying the temperature $T$ or fixing the temperature and varying the chemical potential. We define the temperature by $T / T_{c}=\bar{\mu}_{c} / \bar{\mu}$ and fix $\mu=1$.

Spontaneous symmetry breaking is driven by low temperature or high chemical potential. It triggers a non trivial expectation value for the scalar field without switching on any source $J_{\mathcal{O}}$. For small $\bar{\mu}$ the scalar field is trivial and the gauge equation is solved by $\chi=\bar{\mu}(1-1 / \rho)$ and $\psi=0$. The system is then in the symmetric phase. However, by decreasing the temperature the system becomes unstable towards condensation of the scalar 144, 151. In [2] it was shown that at the critical temperature indeed the lowest quasinormal mode of the scalar field becomes unstable, i. e. it crosses over to the upper half plane.

The free energy density of the system is given by the on-shell renormalized action,

$$
F=-T S_{r e n}=-T\left(\frac{1}{2} \mu n-\int_{r_{H}}^{\infty} d r \frac{r^{2} \psi^{2} \chi^{2}}{f}\right)
$$

The second term vanishes in the absence of a condensate and it works against the phase transition if it is present. In Figure 8.1 the free energies for the symmetric and broken phase are compared. It is clear that for $T<T_{c}$ the condensate solution is always preferred and therefore the system undergoes a second order phase transition to the superconducting phase. Note that the presence of the second scalar plays no role for the phase structure. It simply vanishes in the broken and unbroken phase $\lambda=0$. In order to extract the
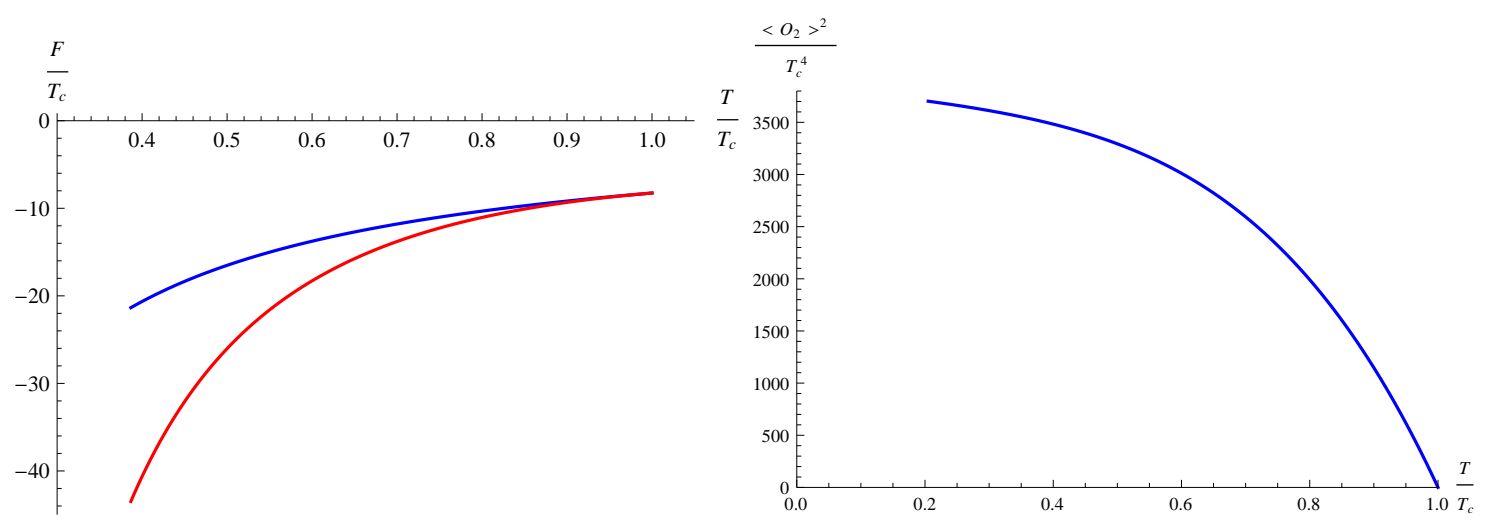

Figure 8.1: (Left) The free energy of the trivial (blue) and condensate (red) background solutions at low temperatures, $T<T_{c}$. (Right) Value of the condensate in the grand canonical ensemble as a function of $T / T_{c}$.

quasinormal mode spectrum, we switch on fluctuations of the background fields

$$
\begin{aligned}
\Psi^{\mathrm{T}} & =(\eta(\rho, t, x), \psi(\rho)+\sigma(\rho, t, x)), \\
A & =\left(\chi(\rho)+a_{t}(\rho, t, x)\right) d t+a_{x}(\rho, t, x) d x .
\end{aligned}
$$

We do not include transverse fluctuations because they decouple from the interesting physical features of the model at hand. 
In the normal phase, i.e. expanding around $\psi(\rho)=0$, the system reduces to the $U(1)$ holographic superconductor studied in [2] with two copies of the scalar fluctuations,

$$
\begin{aligned}
f s^{\prime \prime}+s^{\prime}\left(f^{\prime}+\frac{2 f}{\rho}\right)+\left(\frac{(\chi+\omega)^{2}}{f}-\frac{k^{2}}{\rho^{2}}-m^{2}\right) s & =0, \\
f a_{t}^{\prime \prime}+\frac{2 f}{\rho} a_{t}^{\prime}-\frac{k^{2}}{\rho^{2}} a_{t}-\frac{\omega k}{\rho^{2}} a_{x} & =0, \\
f a_{x}^{\prime \prime}+f^{\prime} a_{x}^{\prime}+\frac{\omega^{2}}{f} a_{x}+\frac{\omega k}{f} a_{t}=0, & =0, \frac{i \omega}{f} a_{t}^{\prime}+\frac{i k}{\rho^{2}} a_{x}^{\prime}=0,
\end{aligned}
$$

where $s$ stands for both $\eta$ and $\sigma$ fluctuations. The equation for the complex conjugate scalar $\bar{s}$ can be obtained by changing the sign of the potential $\chi$ in 8.1.17. The frequency and momentum are related to the physical ones by

$$
\begin{aligned}
\omega & =\frac{3}{4 \pi T} \omega_{p h}, \\
k & =\frac{3}{4 \pi T} k_{p h} .
\end{aligned}
$$

The scalar and gauge fluctuations completely decouple in the symmetric phase. This is a consequence of working in the probe limit. The quasinormal mode spectrum of the $U(1)$ field in the normal phase is just that of an electromagnetic field on an AdS-Sch background. The longitudinal fluctuations contain one hydrodynamic mode, $\omega=-i D k^{2}$, reflecting the diffusive behavior of normal fluids. In physical units $D=3 /(4 \pi T)$, see the discussion of Section 2.3.1. Due to the lack of an energy-momentum tensor for the dual field theory in the probe limit, the diffusion pole is the only hydrodynamic mode in the unbroken phase.

There are two copies of the scalar fluctuations. The quasinormal modes of $\eta$ and $\sigma$ move towards the origin when decreasing the temperature, whereas the modes of $\bar{\eta}$ and $\bar{\sigma}$ have larger masses and widths the smaller the temperature. As we approach the critical temperature $T=T_{c}$, the lowest quasinormal modes of $\eta$ and of $\sigma$ become massless, triggering the phase transition: the scalar field acquires a non trivial vev in order to avoid its fluctuations to become tachyonic. By symmetry we can choose the condensate to reside completely in the $\psi$ field. The fluctuations $\sigma$ couple then to the gauge field fluctuations just as in 2]. Therefore the QNM spectrum in this sector contains a Goldstone mode with linear dispersion relation $\omega= \pm v_{s} k+O\left(k^{2}\right)$. This is the usual type I Goldstone boson associated with the breaking of the gauge $U(1)$ symmetry. As aforementioned, it can be interpreted as the sound mode of the dual superfluid in the broken phase. What happens then to the QNMs in the fluctuations of the second scalar $\eta$ ? At the critical temperature there is also an ungapped mode present since its QNM spectrum is simply another copy of the scalar sector. Since there are no operators generating the $S U(2)$ symmetry in the dual field theory standard arguments about the appearance of Goldstone modes do a priori not apply. Three logical possibilities arise then: the mode could become unstable for $T<T_{c}$, it could become gapped again or it stays ungapped, playing the role of an unexpected Goldstone boson for the broken bulk-global $S U(2)$ symmetry. Shortly we will see that the last possibility is realized and that the massless mode of $\eta$ will indeed correspond to a type II Goldstone boson with quadratic dispersion relation, $\omega \propto k^{2}$. 
In the broken phase, the equations of motion read

$$
\begin{aligned}
& 0=f \eta^{\prime \prime}+\eta^{\prime}\left(f^{\prime}+\frac{2 f}{\rho}\right)+\left(\frac{(\chi+\omega)^{2}}{f}-\frac{k^{2}}{\rho^{2}}-m^{2}\right) \eta \\
& 0=f \delta^{\prime \prime}+\delta^{\prime}\left(f^{\prime}+\frac{2 f}{\rho}\right)+\left(\frac{\chi^{2}}{f}+\frac{\omega^{2}}{f}-\frac{k^{2}}{\rho^{2}}-m^{2}\right) \delta-\frac{2 i \omega \chi}{f} \zeta-i \psi\left(\frac{\omega}{f} a_{t}+\frac{k}{\rho^{2}} a_{x}\right), \\
& 0=f \zeta^{\prime \prime}+\zeta^{\prime}\left(f^{\prime}+\frac{2 f}{r}\right)+\left(\frac{\chi^{2}}{f}+\frac{\omega^{2}}{f}-\frac{k^{2} L^{2}}{r^{2}}-m^{2}\right) \zeta+\frac{2 i \omega \chi}{f} \delta+\frac{2 \chi \psi}{f} a_{t} \\
& 0=f a_{t}^{\prime \prime}+\frac{2 f}{\rho} a_{t}^{\prime}-\left(\frac{k^{2}}{\rho^{2}}+2 \psi^{2}\right) a_{t}-\frac{\omega k}{\rho^{2}} a_{x}-2 i \omega \psi \delta-4 \chi \psi \zeta \\
& 0=f a_{x}^{\prime \prime}+f^{\prime} a_{x}^{\prime}+\left(\frac{\omega^{2}}{f}-2 \psi\right) a_{x}+\frac{\omega k}{f} a_{t}+2 i k \psi \delta \\
& 0=\frac{i \omega}{f} a_{t}^{\prime}+\frac{i k}{\rho^{2}} a_{x}^{\prime}+2 \psi^{\prime} \delta-2 \psi \delta^{\prime}
\end{aligned}
$$

where we have divided $\sigma=\zeta+i \delta$ into real and imaginary part. The system 8.1.24 8.1.28 is again the one studied in [2]. This sector, that also appears in the gauged model that will be presented afterwards, decouples from the additional scalar fluctuation $\eta$. Notice that even if 8.1 .23 is formally the same as in the normal phase, the background $\chi$ is different leading to non trivial features in the $\eta$ sector such as the presence of a massless excitation.

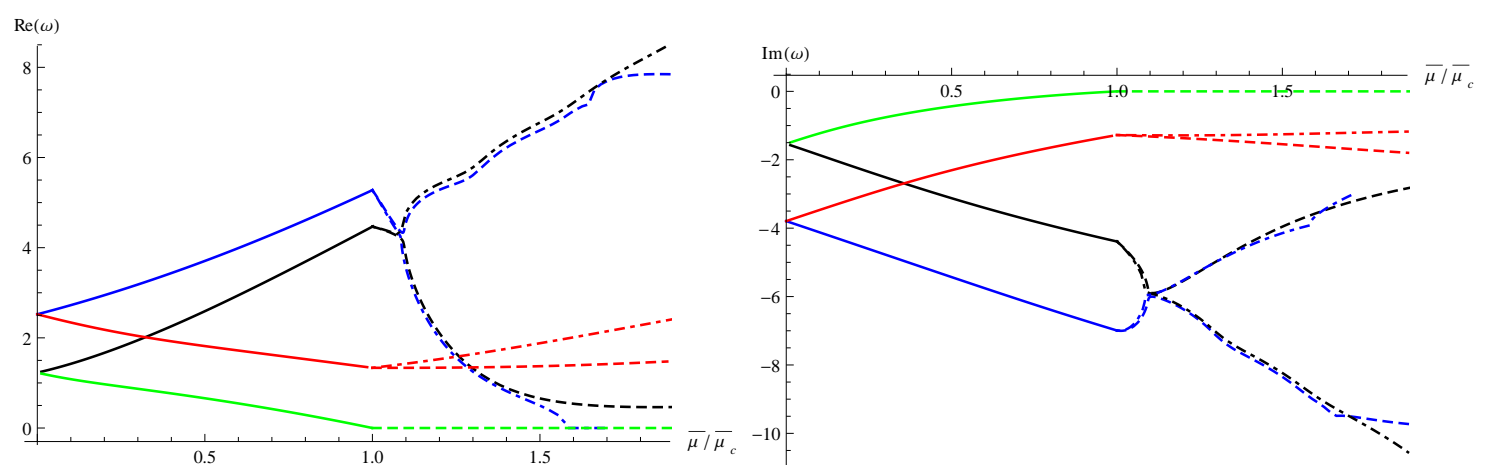

Figure 8.2: Real (left) and imaginary (right) parts of the lowest scalar QNMs as a function of the chemical potential. Solid lines correspond to the unbroken phase. For the broken phase dashed lines stand for modes of the additional scalar while dotdashed lines represent the modes common to the $U(1)$ holographic superconductor.

Figure 8.2 shows the spectrum of quasinormal excitations of the scalar doublet. In the normal phase we have two degenerate copies of the spectrum that partially split after the phase transition. It is clear that the two lowest excitations become massless at the critical chemical potential and then remain massless in the superconducting phase. They can be identified with the two Goldstone bosons at the phase transition. The rest of the excitations remain gapped in the broken phase. Notice that the first $\bar{\eta}$ excitation (dashed black line in figure 8.2 does not follow the expected universal behavior in the broken phase, i.e. it is not linear in $\mu$. This mode is the equivalent of the special gapped mode $\omega_{4}$ in the field theoretical model of section 4.4.1. However, it has already been mentioned that the ungauged model does not satisfy all the theorems about symmetry breaking and therefore deviations from the universal behavior should not be surprising. The behavior of the gapped modes is actually similar to that of the $U(1)$ model modes. In the unbroken 
phase we can distinguish the modes that come from the $s$-type of fluctuations from the ones that come from the complex conjugate $\bar{s}$ fluctuations. The former become lighter whereas the latter become heavier ${ }^{3}$. In the broken phase it is more useful to use real and imaginary parts, at least for the scalar that mixes with the gauge fields fluctuations, i.e. the lower component of the scalar in our conventions. So we can not a priori talk of $s$ and $\bar{s}$ type fluctuations. We still can study to which modes the $s$ and $\bar{s}$ type modes connect to in the broken phase. Here we see an interesting pattern: the $s$ type modes split in the broken phase whereas the $\bar{s}$ type modes stay almost degenerate close to the phase transition (at least at zero momentum). This is surprising given the fact that the fluctuations correspond to two completely different systems, one coming from a single differential equation whereas the others come from a complicated system of coupled equations. However, for small temperatures they split and actually the real part of the lowest one for the $U(1)$ sector goes to zero at a finite temperature. For temperatures below $T \approx 0.63 T_{c}$ it becomes a purely imaginary mode.

Sound mode: There are two massless modes in the broken phase. The first one is the type I Goldstone boson appearing because of the spontaneous breaking of the $U(1)$ gauge symmetry. In [2], it was shown that this mode corresponds to the sound mode of the dual superfluid and that in the hydrodynamic limit it has a linear dispersion relation

$$
\omega_{I}= \pm\left(v_{s} k+\bar{b} k^{2}\right)-i \Gamma_{s} k^{2}
$$

where $v_{s}$ is the speed of sound and $\Gamma_{s}$ is its attenuation. It turns out that the quadratic part of the dispersion relation also has a real component. This component is very small and subleading compared to the linear term that determines the speed of sound. In [2] this real quadratic part has not been studied.
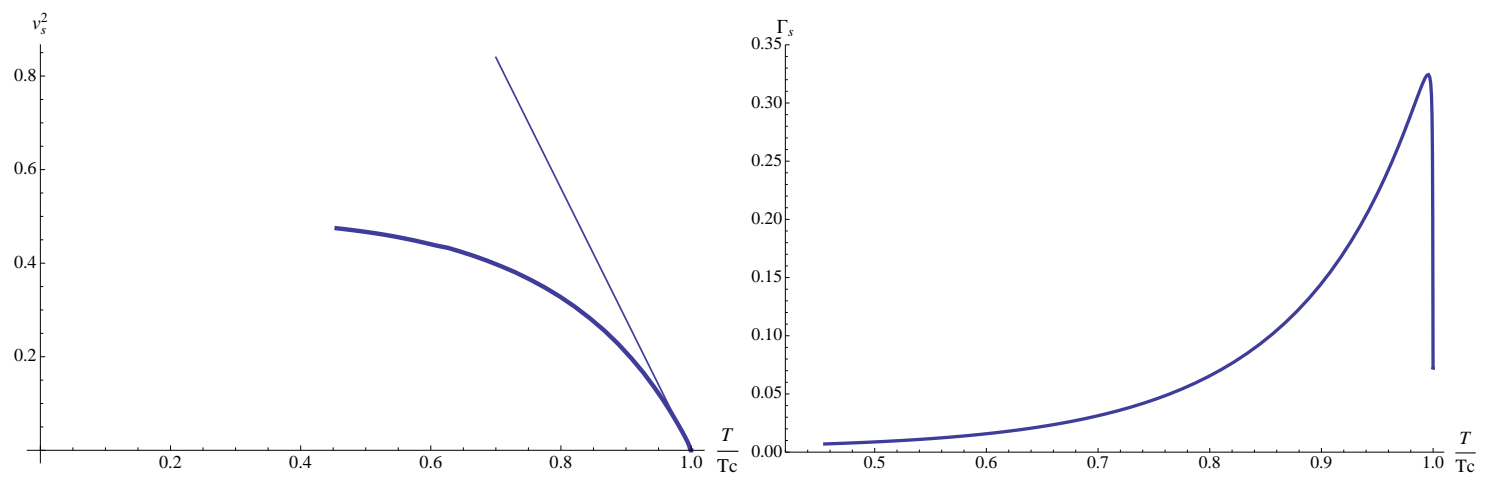

Figure 8.3: Speed of sound and damping for the sound mode. The speed of sound goes to zero at the critical temperature. The damping constant first rises quickly and then falls off again. Precisely at the critical temperature its value is such that the sound modes connect continuously to the scalar modes that become massless there. The peak in the damping constant sits close to the critical temperature and was not resolved in [2].

For very small temperatures the velocity approaches its conformal value $v_{s}^{2}=1 / 2$ while the width goes to zero, see figure 8.3 . Close to the phase transition, the speed of sound

\footnotetext{
${ }^{3}$ This behavior is reversed if we had taken the chemical potential to be negative.
} 
has a mean field behavior as a function of temperature

$$
v_{s}^{2} \approx 2.8\left(1-\frac{T}{T_{c}}\right)
$$

As expected, at $T=T_{c}$ the speed of sound vanishes. This can be traced back to the fact that at the phase transition the total mass $m_{*}^{2}=M^{2}-\mu^{2}$ fulfills $m_{*}^{2}=v^{2}=0$, as expected, and hence the complex scalar field, charged under a $U(1)$ symmetry, becomes massless.

Indeed, one can write down the effective action, analogous to 4.4.21), for a complex scalar field with mass $M$, in the presence of a chemical potential for a $U(1)$ symmetry that is spontaneously broken. The excitations on top of the $U(1)$-breaking background have a dispersion relation equal to 4.4.25)-4.4.26, being (4.4.25 the type I Goldstone boson. It is a general feature of these linear sigma models that the coefficient in front of the linear term in the momentum depends on $m_{*}^{2}$, as can be explicitly checked for the case at hand (see (4.4.25)). Therefore, at the phase transition the leading term in the dispersion relation is of $O\left(k^{2}\right)$; this effect can be seen very clearly with numerical methods, as shown in Figure 8.4. Since the quasinormal mode spectrum has to vary continuously through the second order phase transition the real and complex coefficients of the $k^{2}$ term have to coincide at $T=T_{c}$ with the ones obtained from the massless scalars in the unbroken phase. Numerically we find $\bar{b}\left(T_{c}\right)=0.22$ and $\Gamma_{s}\left(T_{c}\right)=0.071$.

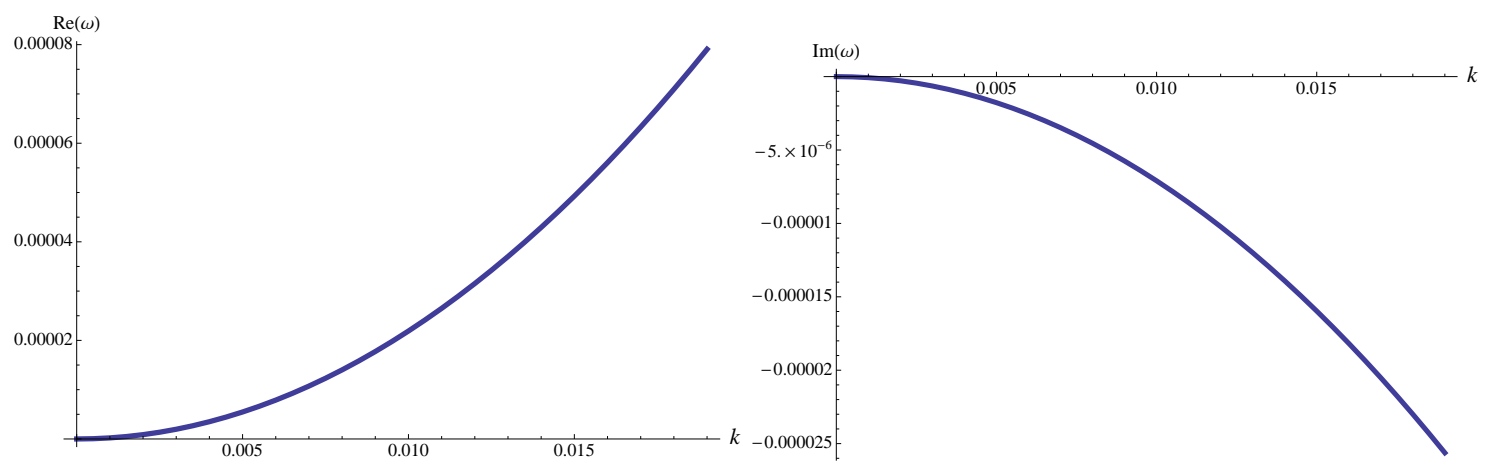

Figure 8.4: Dispersion relations of $\operatorname{Re} \omega$ (left) and $\operatorname{Im} \omega$ (right) at $T=T_{c}$ for the type I Goldstone boson in the system studied by [2]. The behavior Re $\omega \sim k$ becomes quadratic right at this temperature: $\operatorname{Re} \omega=\bar{b} k^{2}$. The coefficient is $\bar{b}=0.22$, which in turn matches the value that one finds if approaches $T_{c}$ from above (i.e. from the unbroken phase).

Pseudo diffusion mode: In the unbroken phase our model has only one hydrodynamic mode, the diffusion mode $\omega=-i D k^{2}+O\left(k^{4}\right)$ with $D=3 /(4 \pi T)$ in physical units. The shear and normal sound modes have their origin in the metric fluctuations and therefore are absent in the decoupling limit we are studying. The phase transition to the broken phase is second order. For the spectrum of quasinormal modes this implies that the modes of the broken and unbroken phase must connect continuously through the phase transition. In the case of the diffusion mode there must therefore exist a quasinormal mode with purely imaginary frequency. Hydrodynamics implies however that the only ungapped modes are the sound modes corresponding to the type I Goldstone mode. Not too far from the phase transition, i.e. for $T \lesssim T_{c}$ the diffusion mode of the broken phase must develop into a mode with dispersion relation

$$
\omega=-i \gamma(T)-i D(T) k^{2}+O\left(k^{4}\right)
$$


as shown in Figure 8.5 .

We might say that the diffusion mode develops a gap in the broken phase and becomes what has been called a pseudo diffusion mode in [2]. Precisely at zero momentum $k=0$ this gapped pseudo diffusion mode explains the observation made in [152] on the late time response of holographic superconductors. For temperatures $T \lesssim T_{c}$ the pseudo diffusion mode is the mode that lies closest to the real axis and therefore it dominates the long time response to any perturbation, such as the quenches studied in [152]. It follows that the order parameter shows a purely exponential decay since this mode does not have a real frequency. The existence of that mode can ultimately be traced back to the universality of the diffusion mode in the unbroken phase. We expect therefore the pseudo diffusion mode to be a universal feature of a wide class of superfluids (not necessarily holographic ones).
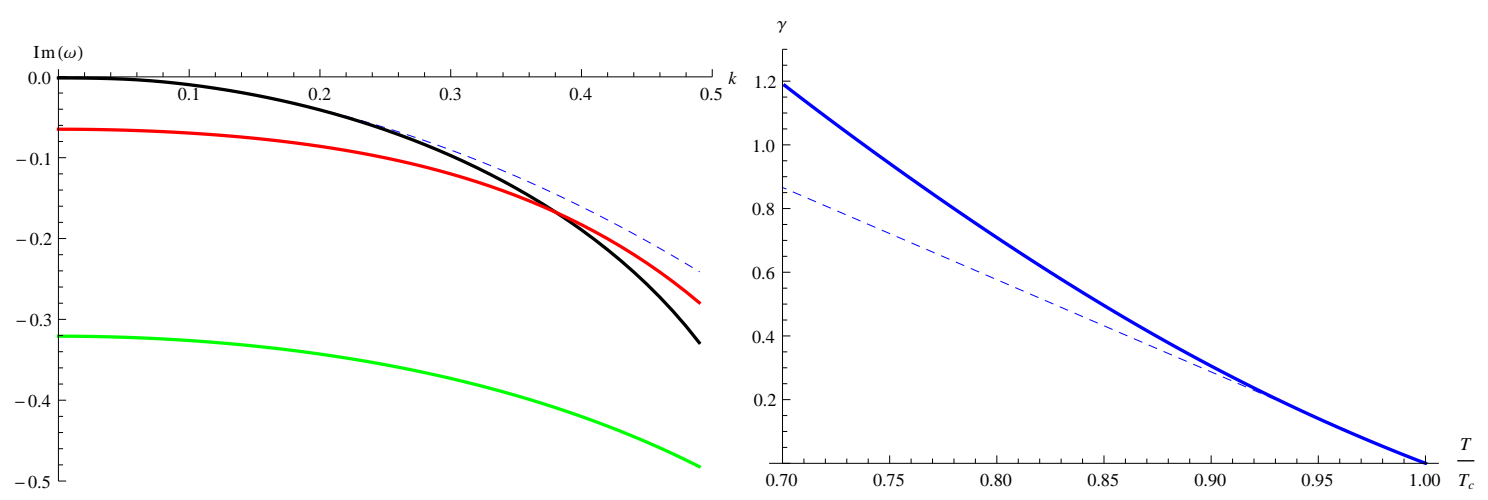

Figure 8.5: (Left) Dispersion relation of the gapped pseudo diffusion mode in the broken phase for three different temperatures. The gap widens as the temperature is lowered. (Right) Gap $\gamma$ as a function of the reduced temperature $T / T_{c}$. As one approaches the critical temperature from below the gap vanishes linearly.

The gap $\gamma$ grows as the temperature decreases. On the other hand there are quasinormal modes (connecting to the QNMs in the scalar sector of the unbroken phase) whose imaginary part is only weakly dependent on the temperature. At a certain crossover temperature $T_{*}$ the gap of the pseudo diffusion mode is bigger than the imaginary part of these modes, as shown in Figure 8.6. Then the response pattern changes from a purely exponential decay to an exponentially damped oscillation. Numerically we find that the crossover temperature is $T_{*}=0.69 T_{c}$. Such crossover changes in the long term response appear frequently in the details of the quasinormal mode spectrum of holographic field theories, $[51,153,154$. In fact this purely exponential decay applies not only to the order parameter but to all operators that correspond to the fields participating in the fluctuation system 8.1.24 8.1.28, e.g. charge density or $x$-component of the current.

For finite momentum the response pattern is expected to be different however. Now one also has to take into account the sound mode. While precisely at zero momentum the sound mode, i.e. the Goldstone mode, degenerates to a constant phase change of the condensate at small but non-zero momentum the long time response should be dominated by the complex frequencies 8.1.29. If one looks however only to the response in the gauge invariant order parameter the Goldstone modes, being local phase rotations of the order parameter, are projected out.

\footnotetext{
${ }^{4}$ This is lower than in the model of $[152$. The difference is presumably due to the fact that we work in the decoupling limit.
} 

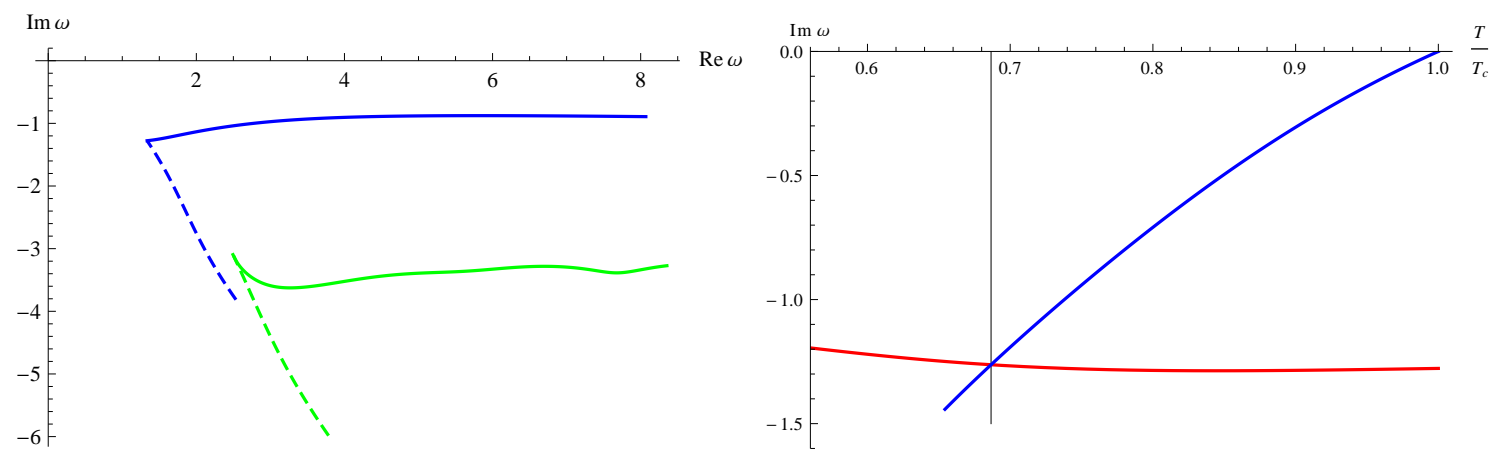

Figure 8.6: (Left) Continuation of the second and third scalar QNM into the broken phase. The real part grows as the temperature is lowered whereas the imaginary part shows very little dependence on $T$. (Right) The gap $\gamma$ (blue line) and the imaginary part of the lowest (scalar) mode fluctuation (red line) in the broken phase are shown as function of $T / T_{c}$. At $T_{*} \approx 0.69 T_{c}$ the imaginary parts cross. For lower temperatures the late time response is not dominated anymore by the pseudo diffusion mode and consequently is in form of a exponentially decaying oscillation.

Type II Goldstone mode: The second massless mode is the Goldstone boson associated with the breaking of the bulk-global $S U(2)$ symmetry. It can be fit to a quadratic dispersion relation of the form

$$
\omega_{I I}= \pm b k^{2}-i c k^{2}+O\left(k^{4}\right),
$$

in the long wavelength limit. Therefore it has the characteristic of a type II Goldstone mode. In Figure 8.7 the dispersion relation for the $\eta$ massless mode is shown for various temperatures as well as its fit to the hydrodynamic form. It is clear that there is a good agreement in the regime of validity of the low energy limit.

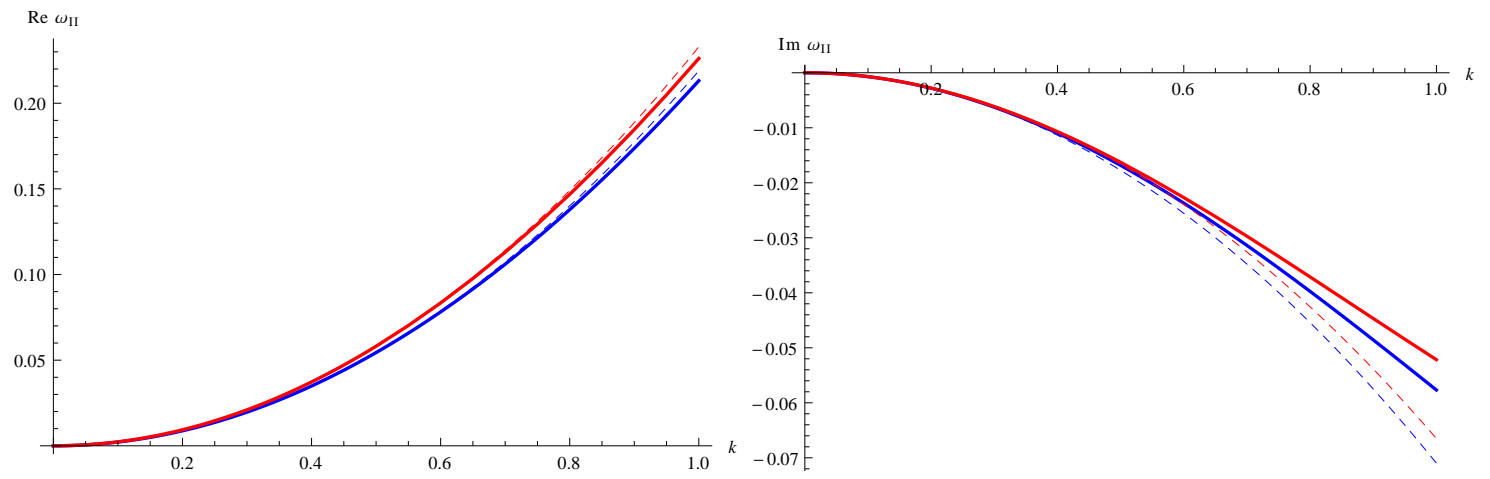

Figure 8.7: Real (left) and imaginary (right) parts of the type II Goldstone mode as a function of the momentum for $T / T_{c}=0.9998$ (blue) and $T / T_{c}=0.704$ (red). The solid lines correspond to the numerical result while the dashed lines are the quadratic fit to a dispersion relation $\omega_{I I}=b k^{2}-i c k^{2}$.

The coefficients in the hydrodynamic dispersion relation 8.1 .32 as a function of the temperature are shown in Figure 8.8. Close to the phase transition they have a linear 
dependence in the reduced temperature

$$
\begin{aligned}
& b(T)=0.22+0.049\left(1-\frac{T}{T_{c}}\right), \\
& c(T)=0.071-0.0014\left(1-\frac{T}{T_{c}}\right) \quad \text { near } T_{c} .
\end{aligned}
$$

Notice that at the phase transition the sound mode and the type II Goldstone must behave in the same way due to continuity of the modes through the phase transition and the fact that they are degenerate in the normal phase. In fact, at the transition $b=\bar{b}=0.22$ and $c=\Gamma_{s}=0.071$, values that of course coincide with those of the lowest scalar mode in the normal phase. On the other hand, it is interesting to notice that in the broken phase the behavior of the coefficients of the type II Goldstone is completely different from that of the coefficients of the sound of the superfluid. Unlike the sound velocity, that vanishes at the phase transition, the coefficient $b$ of the type II Goldstone mode takes a finite value at the critical temperature. This result of course persists for the gauged model. The attenuation on the other hand, as it happens for the $U(1)$ sector, has a finite value at the phase transition and then decreases with temperature, reflecting the fact that the fluid is more ideal the lower the temperature.
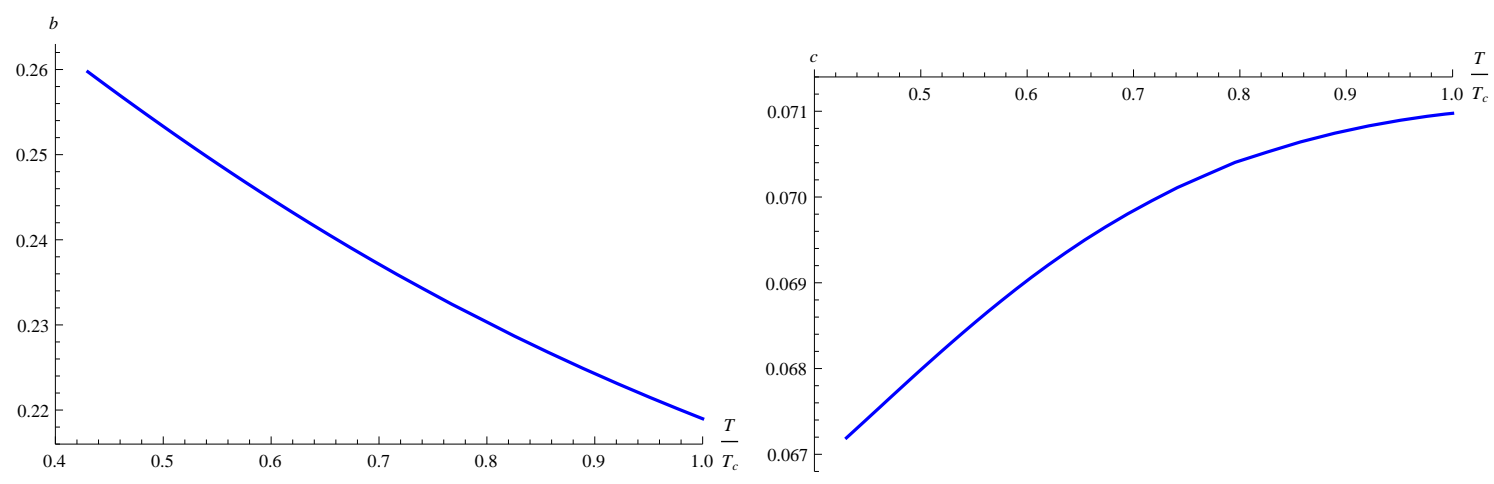

Figure 8.8: Coefficients of the type II Goldstone mode dispersion relation $\omega_{I I}=b k^{2}-i c k^{2}$, as a function of the temperature. Dependence with temperature is very mild.

\subsection{The gauged model}

Let us now discuss the fully gauged model. Consider the following Lagrangian for a complex scalar field living in the fundamental representation of a $U(2)$ gauge symmetry of the bulk,

$$
S=\int \sqrt{-g} \mathcal{L}=\int d^{4} x \sqrt{-g}\left(-\frac{1}{4} F^{\mu \nu c} F_{\mu \nu}^{c}-m^{2} \Psi^{\dagger} \Psi-\left(D^{\mu} \Psi\right)^{\dagger} D_{\mu} \Psi\right)
$$

where

$$
\Psi=\left(\begin{array}{l}
\lambda \\
\psi
\end{array}\right), \quad A_{\mu}=A_{\mu}^{c} T_{c}, \quad D_{\mu}=\partial_{\mu}-i A_{\mu},
$$

and $c=0,1,2,3$ is the color index. The field $\Psi$ plays the role of the condensate. The expectation value of its dual operator thus triggers the spontaneous breaking of the $U(2)$ 
global symmetry of the boundary theory. For simplicity, we set $\lambda=0$ in the background. $T_{c}$ are the generators of $U(2)$ :

$$
T_{0}=\frac{1}{2} \mathbb{I}, \quad T_{i}=\frac{1}{2} \sigma_{i} .
$$

Notice that we are again working in the probe limit, so the background metric is taken to be the Schwarzschild-AdS black brane of 8.1.3. On the other hand, the gauge field is now

$$
A_{0}^{(0)} \equiv \Phi(r), \quad A_{0}^{(3)} \equiv \Theta(r) .
$$

The rest of the components of the gauge field being zero. As in the previous section, we will use dimensionless coordinates defined by the rescaling given in 8.1.4.

The equations of motion for our ansatz are

$$
\begin{aligned}
\psi^{\prime \prime}+\left(\frac{f^{\prime}}{f}+\frac{2}{\rho}\right) \psi^{\prime}+\frac{(\Phi-\Theta)^{2}}{4 f^{2}} \psi-\frac{m^{2}}{f} \psi & =0, \\
\Phi^{\prime \prime}+\frac{2}{\rho} \Phi^{\prime}-\frac{\psi^{2}}{2 f}(\Phi-\Theta) & =0, \\
\Theta^{\prime \prime}+\frac{2}{\rho} \Theta^{\prime}+\frac{\psi^{2}}{2 f}(\Phi-\Theta) & =0 .
\end{aligned}
$$

Notice that from 10.1.7 it follows that we can not simply switch on $\Phi$ without also allowing for a non-trivial $\Theta$. We are of course only interested in switching on a chemical potential in the overall $U(1)$, and therefore we will impose $\Theta(\rho \rightarrow \infty)=0$ and allow for a finite boundary value of $\Phi$.

The coupled system of equations above can be simplified by defining $\chi \equiv \frac{1}{2}(\Phi-\Theta)$ and $\xi \equiv \frac{1}{2}(\Phi+\Theta)$. Using 10.1 .6 and 10.1.7), we see that the resulting equations for these fields are 5

$$
\begin{aligned}
\Psi^{\prime \prime}+\left(\frac{f^{\prime}}{f}+\frac{2}{\rho}\right) \Psi^{\prime}+\frac{\chi^{2}}{f^{2}} \Psi-\frac{m^{2}}{f} \Psi & =0 \\
\chi^{\prime \prime}+\frac{2}{\rho} \chi^{\prime}-\frac{2 \Psi^{2}}{f} \chi & =0 \\
\xi^{\prime \prime}+\frac{2}{\rho} \xi^{\prime} & =0
\end{aligned}
$$

where we have redefined $\psi \rightarrow \sqrt{2} \Psi$. As usual we choose the boundary conditions $\chi(\rho=$ $1)=0, \xi(\rho=1)=0$ along with regularity of $\Psi$. Having a dual field theory with only one finite chemical potential switched on, implies that $\chi$ and $\xi$ must take the same non trivial value at the boundary in order to ensure that $\Theta$ vanishes asymptotically. Notice that $\xi$ decouples completely. The remaining system (8.2.41)-10.1.8) is again the background found for the widely studied s-wave $U(1)$ holographic superconductor. Therefore, the background of the $U(2)$ gauge model contains the Abelian superconductor plus a decoupled conserved $U(1)$ sector.

The field $\chi$ lies in the direction of one of the broken generators, which is the linear combination $\frac{1}{2}\left(T_{3}-T_{0}\right)$, whereas $\xi$ lies in the direction of the preserved $U(1)$ given by $\frac{1}{2}\left(T_{3}+T_{0}\right)$.

\footnotetext{
${ }^{5}$ These equations of motion correspond to the probe limit of the system studied in 155 as a dual of superconductors with chemical potential imbalance. Notice however that in 155 the gauge symmetry was $U(1) \times U(1)$ instead of $U(2)$ as in the present setup.
} 
The asymptotic expansion of the fields near the conformal boundary reads

$$
\begin{gathered}
\chi=\bar{\mu}_{\chi}-\frac{\bar{n}_{\chi}}{\rho}+O\left(\frac{1}{\rho^{2}}\right), \\
\xi=\bar{\mu}_{\xi}-\frac{\bar{n}_{\xi}}{\rho}+O\left(\frac{1}{\rho^{2}}\right), \\
\Psi=\frac{\psi_{1}}{\rho}+\frac{\psi_{2}}{\rho^{2}}+O\left(\frac{1}{\rho^{3}}\right) .
\end{gathered}
$$

The map of the various coefficients in the previous equations to the boundary conditions is $\bar{\mu}_{\chi}=\bar{\mu}_{\xi}=\bar{\mu}$. We will again focus in the $\mathcal{O}_{2}$ theory exclusively, henceforth we will demand $\psi_{1}=0$.

Equations (8.2.41)-(10.1.8) allow for solutions with a non-vanishing condensate, and therefore $\frac{1}{2}\left(T_{3}-T_{0}\right)$ will be spontaneously broken. This solution must be found numerically, since the system is non-linear. However, 8.2.43 does have an analytic solution

$$
\xi=\bar{\mu}\left(1-\frac{1}{\rho}\right)
$$

and thus $\bar{n}_{\xi}=\bar{\mu}$.

When the symmetry is not broken, $\Psi=0$, the equation for $\chi$ has of course

$$
\chi=\bar{\mu}\left(1-\frac{1}{\rho}\right)
$$

as a solution as well. Therefore, in the unbroken phase

$$
\begin{aligned}
& \Theta=0, \\
& \Phi=2 \bar{\mu}\left(1-\frac{1}{\rho}\right) .
\end{aligned}
$$

This behavior reflects the fact that $T_{3}$ is completely independent from $T_{0}$ in the unbroken phase. However, once we switch on the condensate, the interplay between $T_{3}$ and $T_{0}$ (recall that the remaining symmetry is a combination of the two) makes it impossible to set only one of the fields to zero.

Finally, let us mention that in order to relate the dimensionless parameters with the physical ones, we need to apply the same dictionary 8.1.10 -8.1.13 used for the ungauged model.

\subsubsection{Charge Density in the broken phase}

According to 108,112 we can expect the presence of type II Goldstone modes if the broken symmetry generators fulfill

$$
\left\langle\left[Q_{a}, Q_{b}\right]\right\rangle=B_{a b}
$$

with at least one $B_{a b} \neq 0$. In our case we have $\left[Q_{1}, Q_{2}\right]=i Q_{3}$. Therefore in the broken phase we are interested in a non-vanishing expectation value for the charge density operator $\left\langle Q_{3}\right\rangle=n_{\Theta}$. As we argued previously, in the unbroken phase we necessarily have $\Theta(r)=0$. This happened since both $\chi$ and $\xi$ obey the same differential equation and the integration constants had to be set equal in order to do not switch on a source for $\Theta$. Now we would like to find out whether or not an expectation value for $\Theta$ will be spontaneously generated in the broken phase. 
Independently of the phase the field associated to the unbroken combination of generators is given by (8.2.47). Since $\Theta=\xi-\chi$, then

$$
\bar{n}_{\Theta}=\bar{\mu}-\bar{n}_{\chi} .
$$

Hence, what we want to check is the difference between the leading and the subleading coefficients of $\chi$ as a function of the temperature. The numerical result is shown in Figure 8.9 ,

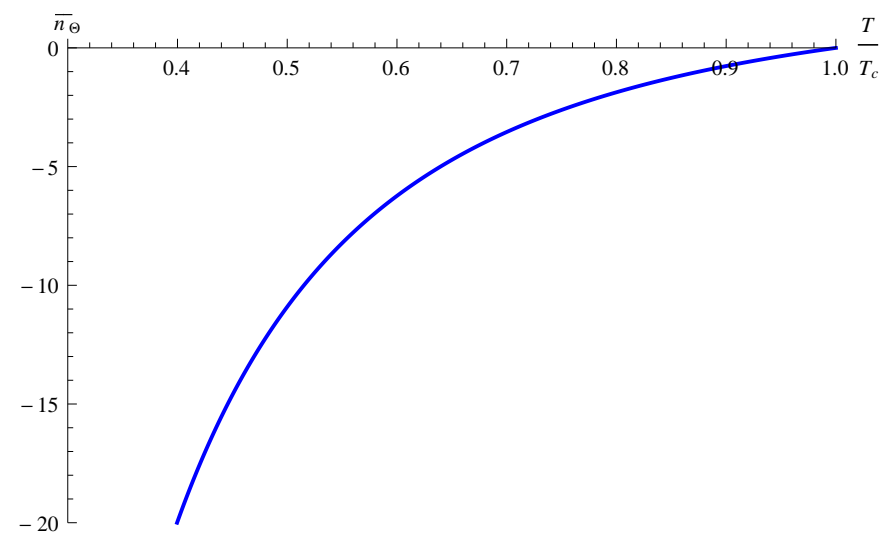

Figure 8.9: Charge density of $\Theta, \bar{n}_{\Theta}$, as a function of the temperature $T / T_{c}$.

So we conclude that precisely at $T \leq T_{c}$ this difference is switched on and an expectation value for $\left\langle Q_{3}\right\rangle$ appears. This can be taken as a clear indication for the appearance of type II Goldstone bosons in the spectrum.

\subsubsection{Fluctuations of the gauged model}

In order to study the quasinormal spectrum and the conductivities of the system, we switch on longitudinal perturbations on top of the background, so that

$$
\begin{aligned}
\hat{\Psi}^{T} & =(\eta(t, \rho, x), \Psi(\rho)+\sigma(t, \rho, x)), \\
A^{(0)} & =\left(\Phi(\rho)+a_{t}^{(0)}(t, \rho, x)\right) d t+a_{x}^{(0)}(t, \rho, x) d x, \\
A^{(1)} & =a_{t}^{(1)}(t, \rho, x) d t+a_{x}^{(1)}(t, \rho, x) d x, \\
A^{(2)} & =a_{t}^{(2)}(t, \rho, x) d t+a_{x}^{(2)}(t, \rho, x) d x, \\
A^{(3)} & =\left(\Theta(\rho)+a_{t}^{(3)}(t, \rho, x)\right) d t+a_{x}^{(3)}(t, \rho, x) d x .
\end{aligned}
$$

\section{Perturbations in the Unbroken Phase}

In the normal phase, the background value of the condensate vanishes. Moreover, we have $\Theta(\rho)=0$. The equations of motion for the perturbations read

$$
\begin{aligned}
s^{\prime \prime}+s^{\prime}\left(\frac{f^{\prime}}{f}+\frac{2}{\rho}\right)+\left(\frac{\left(\frac{\Phi}{2}+\omega\right)^{2}}{f^{2}}-\frac{k^{2}}{f \rho^{2}}-\frac{m^{2}}{f}\right) s & =0, \\
a_{t}^{\prime \prime(c)}+\frac{2}{\rho} a_{t}^{\prime(c)}-\frac{\omega k}{f \rho^{2}} a_{x}^{(c)}-\frac{k^{2}}{f \rho^{2}} a_{t}^{(c)} & =0, \\
a_{x}^{\prime \prime(c)}+\frac{f^{\prime}}{f} a_{x}^{\prime(c)}+\frac{\omega^{2}}{f^{2}} a_{x}^{(c)}+\frac{\omega k}{f^{2}} a_{t}^{(c)} & =0, \\
\frac{\omega}{f} a_{t}^{(c)}+\frac{k}{\rho^{2}} a_{x}^{\prime(c)} & =0,
\end{aligned}
$$


where $s \in\{\eta, \sigma\}$. Since the color indices do not see each other the system is the same one as 8.1.17 8.1.20 except that there are four copies of the gauge field fluctuations. Due to the chosen normalization of the $U(2)$ generators the gauge field background $\Phi$ enters with an additional factor $\frac{1}{2}$ compared to 8.1 .17 . The quasinormal mode spectrum is the same as the one of the holographic s-wave superconductor [2] except that the scalar modes are doubly degenerate and the gauge field modes are fourfold degenerate. In particular there are four copies of the hydrodynamic diffusion mode $\omega=-i D k^{2}$.

\section{Perturbations in the Broken Phase}

The equations of motion in the broken phase decouple in two sets: one mixing the $(0)-(3)$ colors of the gauge field and $\sigma$ fluctuations and the other mixing the (1) - (2) colors and the $\eta$ fluctuations.

Writing $\sigma=\zeta+i \delta$, the equations of the $(0)-(3)$ sector are

$$
\begin{aligned}
0= & \left.f \zeta^{\prime \prime}+\left(f^{\prime}+\frac{2 f}{\rho}\right) \zeta^{\prime}+\left(\frac{\omega^{2}}{f}+\frac{\chi^{2}}{f}-\frac{k^{2}}{\rho^{2}}-m^{2}\right) \zeta+\frac{2 i \omega \chi}{f} \delta+\left(a_{t}^{(0)}-a_{t}^{(3)}\right) \Psi \delta \dot{f}_{f}, 62\right) \\
0= & f \delta^{\prime \prime}+\left(f^{\prime}+\frac{2 f}{\rho}\right) \delta^{\prime}+\left(\frac{\omega^{2}}{f}+\frac{\chi^{2}}{f}-\frac{k^{2}}{\rho^{2}}-m^{2}\right) \delta-\frac{2 i \omega \chi}{f} \zeta+i \Psi \omega \frac{a_{t}^{(3)}-a_{t}^{(0)}}{2 f}+ \\
& +i \Psi k \frac{a_{x}^{(3)}-a_{x}^{(0)}}{2 \rho^{2}}, \\
0= & f a_{t}^{\prime \prime(0)}+\frac{2 f}{\rho} a_{t}^{\prime(0)}-\left(\Psi^{2}+\frac{k^{2}}{\rho^{2}}\right) a_{t}^{(0)}-\frac{\omega k}{\rho^{2}} a_{x}^{(0)}+\Psi^{2} a_{t}^{(3)}-4 \zeta \Psi \chi-2 i \omega \Psi \delta,(8.2 .64) \\
0= & f a_{x}^{\prime \prime(0)}+f^{\prime} a_{x}^{\prime(0)}+\left(\frac{\omega^{2}}{f}-\Psi^{2}\right) a_{x}^{(0)}+\frac{\omega k}{f} a_{t}^{(0)}+\Psi^{2} a_{x}^{(3)}+2 i k \delta \Psi, \\
0= & f a_{t}^{\prime \prime(3)}+\frac{2 f}{\rho} a_{t}^{\prime(3)}-\left(\Psi^{2}+\frac{k^{2}}{\rho^{2}}\right) a_{t}^{(3)}-\frac{\omega k}{\rho^{2}} a_{x}^{(3)}+\Psi^{2} a_{t}^{(0)}+4 \zeta \Psi \chi+2 i \omega \Psi \delta,(8.2 .66) \\
0= & f a_{x}^{\prime \prime(3)}+f^{\prime} a_{x}^{\prime(3)}+\left(\frac{\omega^{2}}{f}-\Psi^{2}\right) a_{x}^{(3)}+\frac{\omega k}{f} a_{t}^{(3)}+\Psi^{2} a_{x}^{(0)}-2 i k \delta \Psi, \\
0 & \frac{i k}{\rho^{2}} a_{x}^{\prime(0)}+\frac{i \omega}{f} a_{t}^{\prime(0)}+2 \Psi^{\prime} \delta-2 \Psi \delta^{\prime}, \\
0 & \frac{i k}{\rho^{2}} a_{x}^{\prime(3)}+\frac{i \omega}{f} a_{t}^{\prime(3)}-2 \Psi^{\prime} \delta+2 \Psi \delta^{\prime} .
\end{aligned}
$$

It is trivial to show that by defining new fields $a_{t}^{( \pm)} \equiv \frac{1}{2}\left(a_{t}^{(0)} \pm a_{t}^{(3)}\right)$ and $a_{x}^{( \pm)} \equiv \frac{1}{2}\left(a_{x}^{(0)} \pm a_{x}^{(3)}\right)$ the system further decouples into a coupled system for the scalar fluctuations and $a_{\mu}^{(-)}$ and a background independent set of equations for the $U(1)$ gauge field $a_{\mu}^{(+)}$. The first subsystem reproduces the eoms (8.1.24)-8.1.28) and therefore corresponds to the s-wave $U(1)$ superconductor contained in the $U(2)$ model. On the other hand, the field $a_{\mu}^{(+)}$ corresponds to the preserved gauge symmetry surviving the $U(2) \rightarrow U(1)$ spontaneous symmetry breaking. The quasinormal mode spectrum in this sector is therefore the same one as in [2] plus the QNMs that are stem from a $U(1)$ gauge field in $A d S_{4}$. In particular the hydrodynamic modes in this sector are the sound mode and the diffusion mode of the unbroken $U(1)$.

From now on we will concentrate on the remaining fields. We will call this remaining, inherently non-Abelian sector the (1) - (2) sector and will show that the expected type II 
Goldstone boson resides there. Writing $\eta=\alpha+i \beta$, we find the following equations in the (1) - (2) sector:

$$
\begin{array}{rl}
0= & f \alpha^{\prime \prime}+\left(f^{\prime}+\frac{2 f}{\rho}\right) \alpha^{\prime}+\left(\frac{\omega^{2}}{f}+\frac{(\Phi+\Theta)^{2}}{4 f}-\frac{k^{2}}{\rho^{2}}-m^{2}\right) \alpha+\frac{i \omega(\Phi+\Theta)}{f} \beta- \\
& -i \Psi\left(\frac{k}{2 \rho^{2}} a_{x}^{(2)}+\frac{\omega}{2 f} a_{t}^{(2)}\right)+\frac{\Psi \Phi}{2 f} a_{t}^{(1)}, \\
0= & f \beta^{\prime \prime}+\left(f^{\prime}+\frac{2 f}{\rho}\right) \beta^{\prime}+\left(\frac{\omega^{2}}{f}+\frac{(\Phi+\Theta)^{2}}{4 f}-\frac{k^{2}}{\rho^{2}}-m^{2}\right) \beta-\frac{i \omega(\Phi+\Theta)}{f} \alpha- \\
& -i \Psi\left(\frac{k}{2 \rho^{2}} a_{x}^{(1)}+\frac{\omega}{2 f} a_{t}^{(1)}\right)-\frac{\Phi \Psi}{2 f} a_{t}^{(2)}, \\
0= & f a_{t}^{\prime \prime(1)}+\frac{2 f}{\rho} a_{t}^{\prime(1)}-\left(\Psi^{2}+\frac{k^{2}}{\rho^{2}}\right) a_{t}^{(1)}-\frac{\omega k}{\rho^{2}} a_{x}^{(1)}+i \Theta \frac{k}{\rho^{2}} a_{x}^{(2)}-2 \Phi \Psi \alpha-2 i \omega \Psi(8.2 .72) \\
0= & f a_{x}^{\prime \prime(1)}+f^{\prime} a_{x}^{\prime(1)}+\left(\frac{\omega^{2}}{f}-\Psi^{2}+\frac{\Theta^{2}}{f}\right) a_{x}^{(1)}-2 i \frac{\Theta \omega}{f} a_{x}^{(2)}-i \Theta \frac{k}{f} a_{t}^{(2)}+\frac{\omega k}{f} a_{t}^{(1)} \beta+ \\
& +2 i k \Psi \beta, \\
0= & f a_{t}^{\prime \prime(2)}+\frac{2 f}{\rho} a_{t}^{\prime(2)}-\left(\Psi^{2}+\frac{k^{2}}{\rho^{2}}\right) a_{t}^{(2)}-\frac{\omega k}{\rho^{2}} a_{x}^{(2)}-i \Theta \frac{k}{\rho^{2}} a_{x}^{(1)}+2 \Phi \Psi \beta-2 i \omega \Psi(8.2 .74) \\
0 & f a_{x}^{\prime \prime(2)}+f^{\prime} a_{x}^{\prime(2)}+\left(\frac{\omega^{2}}{f}-\Psi^{2}+\frac{\Theta^{2}}{f}\right) a_{x}^{(2)}+2 i \frac{\Theta \omega}{f} a_{x}^{(1)}+i \Theta \frac{k}{f} a_{t}^{(1)}+\frac{\omega k}{f} a_{t}^{(2)}+ \\
& +2 i k \Psi \alpha, \\
0 & \frac{i k}{\rho^{2}} a_{x}^{\prime(1)}+\frac{i \omega}{f} a_{t}^{\prime(1)}+\frac{1}{f}\left(a_{t}^{\prime(2)} \Theta-a_{t}^{(2)} \Theta^{\prime}\right)+2 \Psi^{\prime} \beta-2 \beta^{\prime} \Psi, \\
0 & \frac{i k}{\rho^{2}} a_{x}^{\prime(2)}+\frac{i \omega}{f} a_{t}^{\prime(2)}-\frac{1}{f}\left(a_{t}^{\prime(1)} \Theta-a_{t}^{(1)} \Theta^{\prime}\right)+2 \Psi^{\prime} \alpha-2 \alpha^{\prime} \Psi .
\end{array}
$$

A comment is in order here. This system of equations could be written in a more compact form by using complex field variables $\eta$ and $a_{t, x}^{(1)} \pm i a_{t, x}^{(2)}$. One has to keep in mind then that the field equations one needs to solve for the QNM spectrum for the complex conjugate fields are not the complex conjugate equations since one has to demand infalling boundary conditions on the fields and on the complex conjugate fields simultaneously. This aspect is somewhat clearer if one works with the (formally) real field variables on paying the price of writing a somewhat lengthy system of equations.

Up to linear order in perturbations, there are three decoupled sectors in the system. Two of them belong to the '( 0$)$ - (3) sector' and they are a copy of the $U(1)$ holographic superconductor, already extensively studied, and the preserved $U(1)$ gauge symmetry. The main features of the spectrum of this sector have already been presented in section 8.1 since it is also a subsector of the ungauged model. On the other hand, the so called '(1) - (2) sector' has not been studied before. The physics in this sector is quite different from the holographic superconductors studied up to now and we will concentrate on it in the rest of this section.

Before studying the quasinormal modes we will focus on a simpler problem, namely the conductivities. 


\subsubsection{Conductivities}

In order to study the conductivities via Kubo formulae, it is enough to solve the linearized equations in the limit $k=0$. The retarded correlators that we are interested in have the form $\mathcal{G}_{\mathcal{R}} \sim\left\langle J_{(c)}^{x}, J_{\left(c^{\prime}\right)}^{x}\right\rangle_{\mathcal{R}}$, with $c, c^{\prime}$ color indices.

We will be applying the prescription of [51] for computing Green functions in the presence of holographic operator mixing. If one has a set of fields $\Phi_{I}$, the two-point correlation functions will be

$$
\mathcal{G}_{I J}=\lim _{\Lambda \rightarrow \infty}\left(\mathcal{A}_{I M} \mathcal{F}_{k}^{M}{ }_{J}(\Lambda)^{\prime}+\mathcal{B}_{I J}\right)
$$

where the matrix $\mathcal{F}_{k}(r)$ is nothing but the bulk-to-boundary propagator for the fields, normalized to be the unit matrix at the boundary. The matrices $\mathcal{A}$ and $\mathcal{B}$ can be read off from the on-shell renormalized action. The corresponding DC conductivities are given by the following Kubo formula

$$
\sigma_{I J}=\lim _{\omega \rightarrow 0}\left(\frac{i}{\omega} \mathcal{G}_{I J}(\omega, 0)\right)
$$

At vanishing momentum the longitudinal components of the gauge field perturbations decouple from the scalar perturbations, as well as from the temporal components of the gauge fields. Moreover, the constraints (eqs. 8.2.68 8.2.69) and 8.2.76 8.2.77) become trivial. Since we know that the system splits into the $(0)-(3)$ and the $(1)-(2)$ sectors we can rearrange the $a_{x}^{(c)}$ fields in two vectors

$$
\Phi_{k(0-3)}^{T}(\rho)=\left(a_{x}^{(0)}(\rho), a_{x}^{(3)}(\rho)\right) \quad \text { and } \quad \Phi_{k(1-2)}^{T}(\rho)=\left(a_{x}^{(1)}(\rho), a_{x}^{(2)}(\rho)\right) .
$$

One can check that in our case the $\mathcal{A}, \mathcal{B}$ matrices take the simple form

$$
\mathcal{A}=-\frac{f(r)}{2} \mathbb{I}, \quad \mathcal{B}=0,
$$

for both sectors. A priori we would have a $4 \times 4$ matrix of conductivities. We know however that the fluctuations in the $(0)-(3)$ and the (1) - (2) sector decouple from each other. Therefore we can restrict ourselves to study two independent $2 \times 2$ matrices of conductivities.

\subsubsection{Conductivities in the $(0)-(3)$ sector}

The $k=0$ equations of motion for $a_{x}^{(0)}$ and $a_{x}^{(3)}$ can be simplified by using the already defined $a_{x}^{(-)}$and $a_{x}^{(+)}$fields. This results in

$$
\begin{aligned}
& 0=f a_{x}^{\prime \prime(+)}+f^{\prime} a_{x}^{(+)}+\frac{\omega^{2}}{f} a_{x}^{(+)} \\
& 0=f a_{x}^{\prime \prime(-)}+f^{\prime} a_{x}^{(-)}+\left(\frac{\omega^{2}}{f}-2 \Psi^{2}\right) a_{x}^{(-)} .
\end{aligned}
$$

We see that the resulting system of equations is now completely decoupled. We only have two diagonal conductivities $\sigma_{++}$and $\sigma_{--}$, corresponding to the unbroken $U(1)$ diffusive sector and a mode which is associated to the broken $U(1)$ coupling to the condensate. The former is the same as in the unbroken phase and of no further interest for us. The latter is again the well-studied $U(1)$ s-wave superconductor. Its conductivity has been already calculated in [3]. To check our numerics we have re-calculated it and in Figure 
8.10 we show its behavior. It coincides completely with [3]. The real part shows the $\omega=0$ delta function characteristic of superconductivity 6 . Numerically this can be seen through the $1 / \omega$ behavior in the imaginary part. The Kramers-Kronig relation (see 111.3 .30$)$ in appendix 11.3 implies then infinite DC conductivity. The real part of the AC conductivity also exhibits a temperature dependent gap.

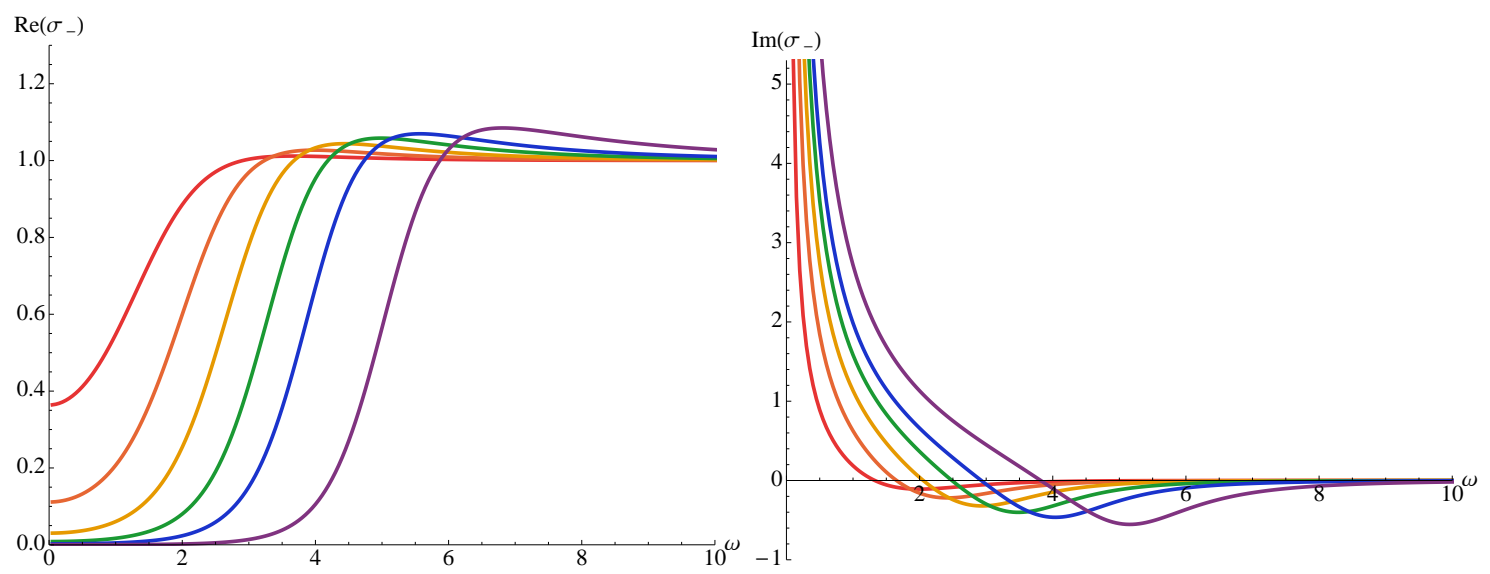

Figure 8.10: Real part (left) and imaginary part (right) of the conductivity as a function of frequency. The plots correspond to temperatures in the range $T / T_{c} \approx 0.91-0.41$, from red to purple. As expected, the plots reproduce the ones of [3].

\subsubsection{Conductivities in the $(1)-(2)$ sector}

The relevant equations for the (1) - (2) sector read

$$
\begin{aligned}
& 0=f a_{x}^{\prime \prime(1)}+f^{\prime} a_{x}^{\prime(1)}+\left(\frac{\omega^{2}}{f}-\Psi^{2}+\frac{\Theta^{2}}{f}\right) a_{x}^{(1)}-2 i \frac{\Theta \omega}{f} a_{x}^{(2)}, \\
& 0=f a_{x}^{\prime \prime(2)}+f^{\prime} a_{x}^{(2)}+\left(\frac{\omega^{2}}{f}-\Psi^{2}+\frac{\Theta^{2}}{f}\right) a_{x}^{(2)}+2 i \frac{\Theta \omega}{f} a_{x}^{(1)} .
\end{aligned}
$$

These equations obey the symmetry

$$
\left(a_{x}^{(1)} \rightarrow a_{x}^{(2)}, a_{x}^{(2)} \rightarrow-a_{x}^{(1)}\right) .
$$

One can see that the fact that $\Theta(1)=0$ implies that $a_{x}^{(1)}(1)$ is independent of $a_{x}^{(2)}(1)$, so, after imposing infalling boundary conditions at the horizon, the parameter space of boundary conditions is two-dimensional, as expected.

In the unbroken phase the system completely decouples

$$
0=f a_{x}^{\prime \prime(c)}+f^{\prime} a_{x}^{\prime(c)}+\frac{\omega^{2}}{f} a_{x}^{(c)} .
$$

\footnotetext{
${ }^{6}$ In general, this behavior is also typical of translation invariant charged media, in which accelerated charges cannot relax. However, working in the probe limit we effectively break translation invariance and therefore the infinite DC conductivity is a genuine sign of superconductivity.
} 
Diagonal Conductivities $\sigma^{11} \& \sigma^{22}$

The diagonal components of the conductivity, $\sigma^{11}$ and $\sigma^{22}$ have the same behavior, as could be anticipated from the equations 8.2.84, 8.2.85. Henceforth, we will only refer to $\sigma^{11}$, but all the conclusions also apply to $\sigma^{22}$.

Figure 8.11 shows the conductivity for several values of the temperature. We find that these conductivities also show delta-function singularities at $\omega=0$.

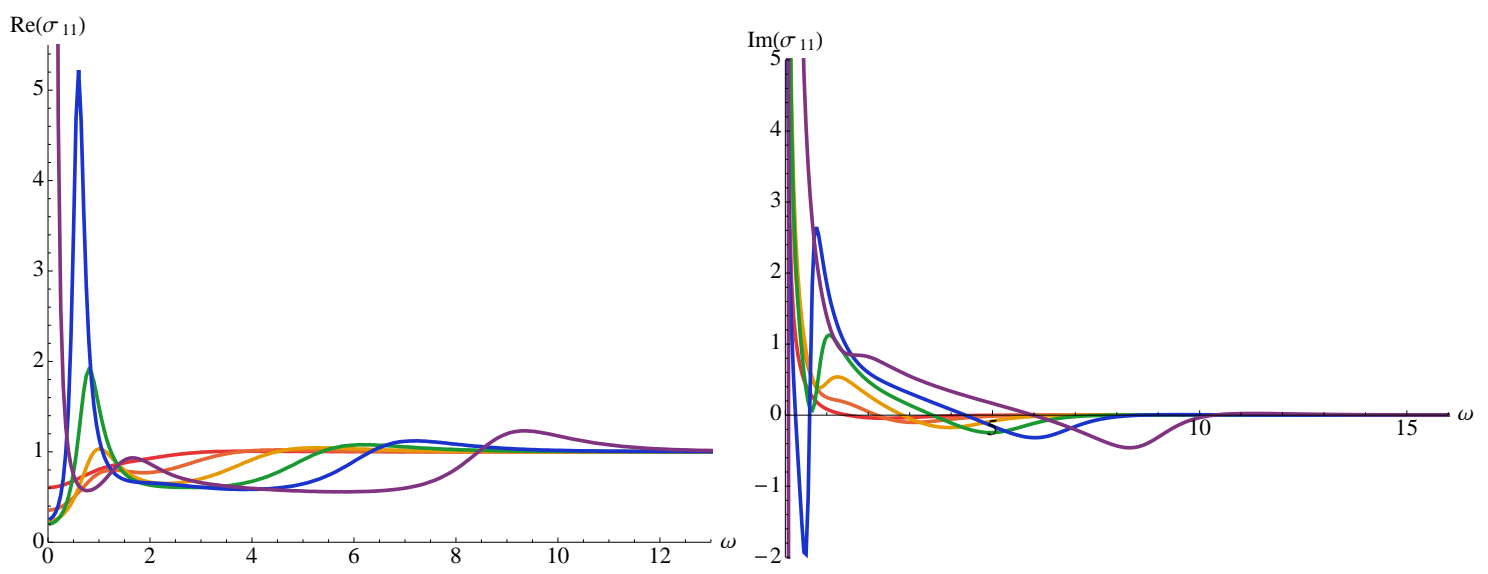

Figure 8.11: Real (left) and imaginary (right) parts of $\sigma^{11}$ versus $\omega$ for five different temperatures chosen in a range $T / T_{c} \approx 0.91-0.41$, from red to purple. $\operatorname{Im}\left(\sigma^{11}\right)$ clearly blows up as $\omega \rightarrow 0$.

The strength of the delta function can also be computed. It is given by the residue of the imaginary part of the conductivity at $\omega=0$,

$$
\lim _{\omega \rightarrow 0} \omega \operatorname{Im}\left(\sigma^{11}\right) \sim n_{s} .
$$

The residue is plotted in Figure 8.12 as a function of $T / T_{c}$. As expected, it starts growing from a zero value. At $T / T_{c} \approx 0.65, n_{s}$ reaches a maximum and starts decreasing fast, changing sign at $T / T_{c}=0.49$. To study $n_{s}$ down to very low temperature we would need to go beyond the probe limit. However, as we will comment below, this behaviour of $n_{s}$ can be understood in light of the QNM spectra.

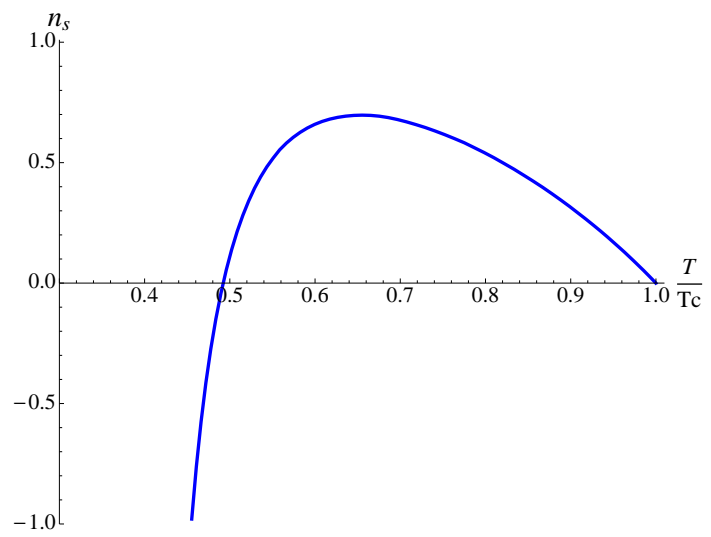

Figure 8.12: Residue at $\omega=0$ as a function of $T / T_{c}$.

Let us look in detail at the behavior of the real part of the conductivity (left plot in figure 8.11). For high enough temperatures the optical conductivity is almost constant, 
$\operatorname{Re}\left(\sigma^{11}\right)=1$, which is expected since in that regime the dynamics is described essentially by (8.2.87). As soon as we decrease the temperature, the onset of the DC conductivity also decreases and only approaches the constant value asymptotically, when $\omega$ becomes large enough and thus the term $\frac{\omega^{2}}{f}$ dominates, turning equations 8.2.84, 8.2.85) approximately into (8.2.87). According to the Ferrell-Glover sum rule, the area missing as we lower the temperature is proportional to $n_{s}$.

Interestingly, at low temperatures the real part of $\sigma^{11}$ starts developing a bump at small values of $\omega(0<\omega \lesssim 2)$. The bump leaves less area for the delta function to cover, which explains why $n_{s}$ starts decreasing approximately at this temperature. Moreover, the appearance of these bumps can be traced back to the fact that for a subleading gauge QNM with small $|\operatorname{Im}(\omega)|, \operatorname{Re} \omega(T)>>\operatorname{Im} \omega(T)$ holds. Hence, the conductivities affected by this mode start developing the reminiscence of a resonance at a particular frequency. We have studied the spectrum of low lying QNM for the gauge sector and found that this mode corresponds in the normal phase to the lowest excitation of $a_{\mu}^{(1,2)}, \omega=-1.5 i$. But it is at lower temperatures where one finds a remarkable fact: at $T / T_{c} \approx 0.395$ the mode becomes unstable, and indeed, as we will see, several physical quantities modify their behavior at that temperature.

Therefore, we expect a new phase transition around $T / T_{c} \approx 0.395$, due entirely to the (1) - (2) sector. Since this phase transition seems to be triggered by an unstable mode in the vector sector it most likely leads to the formation of a p-wave condensate. We will see in Chapter 10 that, indeed, a phase in which an scalar and vector order parameters coexist can be constructed for temperatures below $T / T_{c} \approx 0.395$.

\section{Off-diagonal conductivities $\sigma^{12} \& \sigma^{21}$}

The off-diagonal elements of the conductivity matrix are also related via the symmetry 8.2.86 and therefore obey $\sigma^{12}=-\sigma^{21}$. Therefore, it is enough to comment on $\sigma^{12}$, although the conclusions are valid for both components.

The form of $\sigma^{12}$ is plotted in Figure 8.13 for various different temperatures as a function of frequency. At $T / T_{c}=1$ the system is practically decoupled, so for all temperatures the off-diagonal conductivity goes to zero as $\omega$ increases.

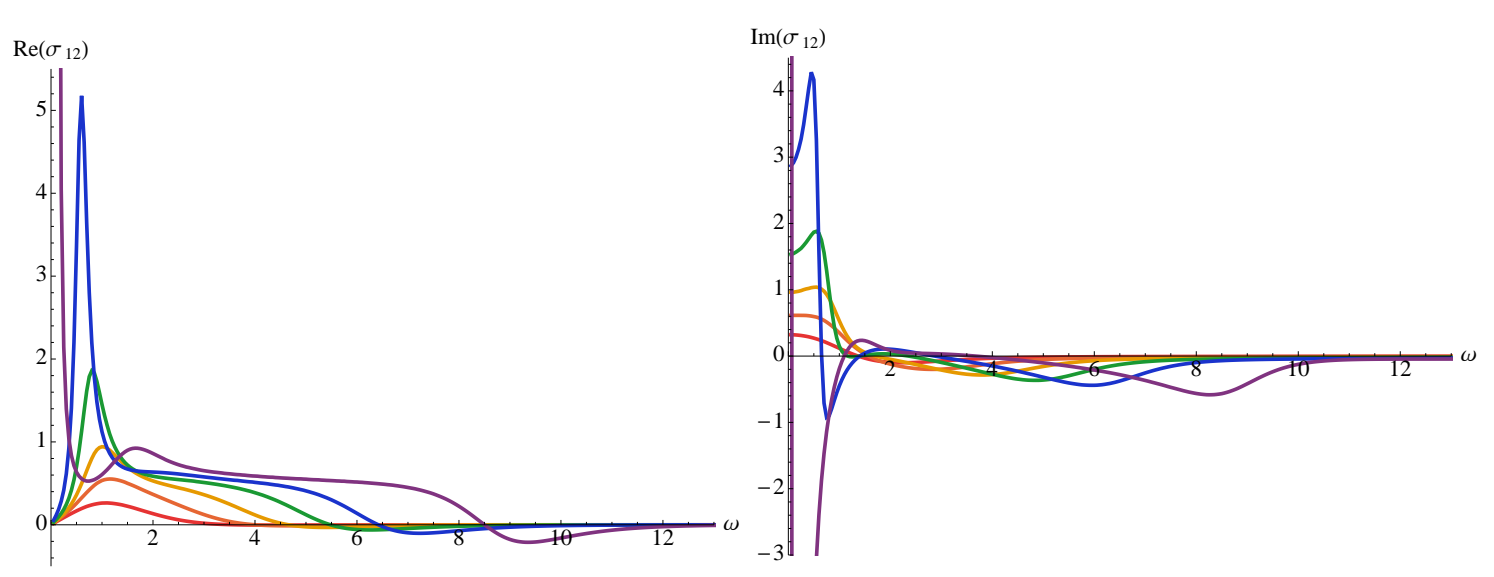

Figure 8.13: Real (left) and imaginary (right) part of $\sigma^{12}$ as a function of $\omega$ for $T / T_{c} \approx$ $0.91-0.41$, from red to purple.

Observe that $\sigma^{12}(\omega)$ behaves as a normal conductivity. Its real part vanishes as $\omega \rightarrow 0$, whereas the imaginary part tends to a constant value. 


\section{Conductivities $\sigma_{+-}$and $\sigma_{-+}$}

It is worth to notice that the equations 8.2.84-8.2.85 decouple if we define a new vector field

$$
\tilde{\varphi}=\left(\begin{array}{c}
A_{+} \\
A_{-}
\end{array}\right)=\left(\begin{array}{cc}
1 & i \\
1 & -i
\end{array}\right)\left(\begin{array}{l}
a_{x}^{(1)} \\
a_{x}^{(2)}
\end{array}\right)=S \varphi .
$$

In this basis, the equations of motion become

$$
0=f A_{ \pm}^{\prime \prime}+f^{\prime} A_{ \pm}^{\prime}+\left(\frac{(\omega \mp \Theta)^{2}}{f}-\Psi^{2}\right) A_{ \pm}
$$

It is easy to check that the relation between the conductivity matrices in the two basis is given by

$$
\tilde{\sigma}=\left(S^{T}\right)^{-1} \sigma S^{-1}
$$

and that only the off-diagonal components of $\tilde{\sigma}$ are non vanishing.

The conductivities $\sigma_{-+}$and $\sigma_{+-}$are represented in Figure 8.14 and 8.15, respectively. The plot of the conductivity $\sigma_{-+}$is particularly suggestive. Besides the superconducting delta of the DC conductivity, it resembles the behavior observed in Graphene [156]. Such a resemblance of the conductivities of holographic superconductors to the one of graphene has been pointed our already in [26]. We emphasize however that the conductivities shown in figure 8.14 have an even closer resemblance to [156. In particular, at small frequencies we see that a Drude-like peak develops. This kind of behavior in metals is usually due to the presence of impurities or lattices, whereas in our case, momentum relaxation would be due to the non-vanishing expectation value of the charge density operator $\left\langle Q_{3}\right\rangle=\bar{n}_{\Theta}$. The resemblance holds for not too low temperatures. When lowering the temperature, a gap opens up as for the (0) - (3) sector. The real part of $\sigma_{+-}$shows the same peak already observed for $\sigma_{11}$ when decreasing the temperature. For temperatures below $T \approx 0.49 T_{c}$, the pole in the imaginary part of both conductivities changes sign. Of course, it corresponds to the temperature at which the residue changes sign. The onset of the DC conductivity at low temperatures grows very fast, becoming divergent at $T / T_{c} \approx 0.395$. The presence of such a pole in the conductivity is related to the appearance of an instability in the spectrum of excitations of the gauge field and therefore with a phase transition to another superconducting phase, as already discussed.

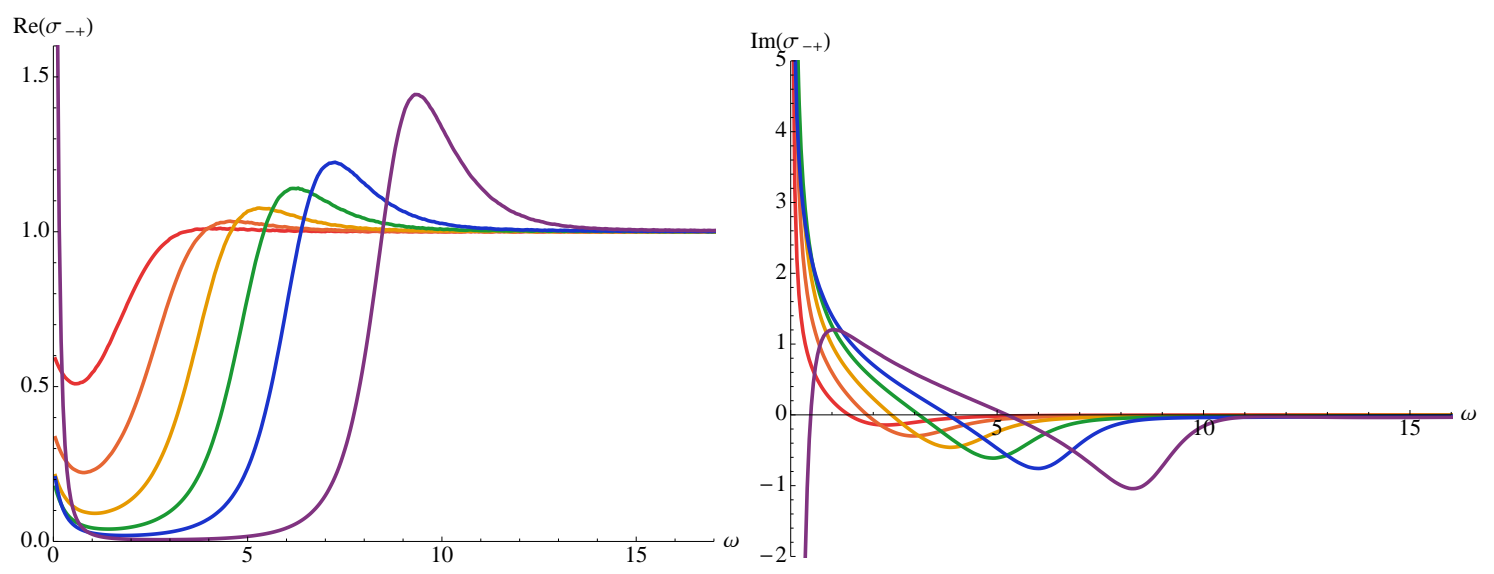

Figure 8.14: Real (left) and imaginary (right) part of the conductivity $\sigma_{-+}$for temperatures in the range $T / T_{c} \approx 0.91-0.41$, from red to purple. 

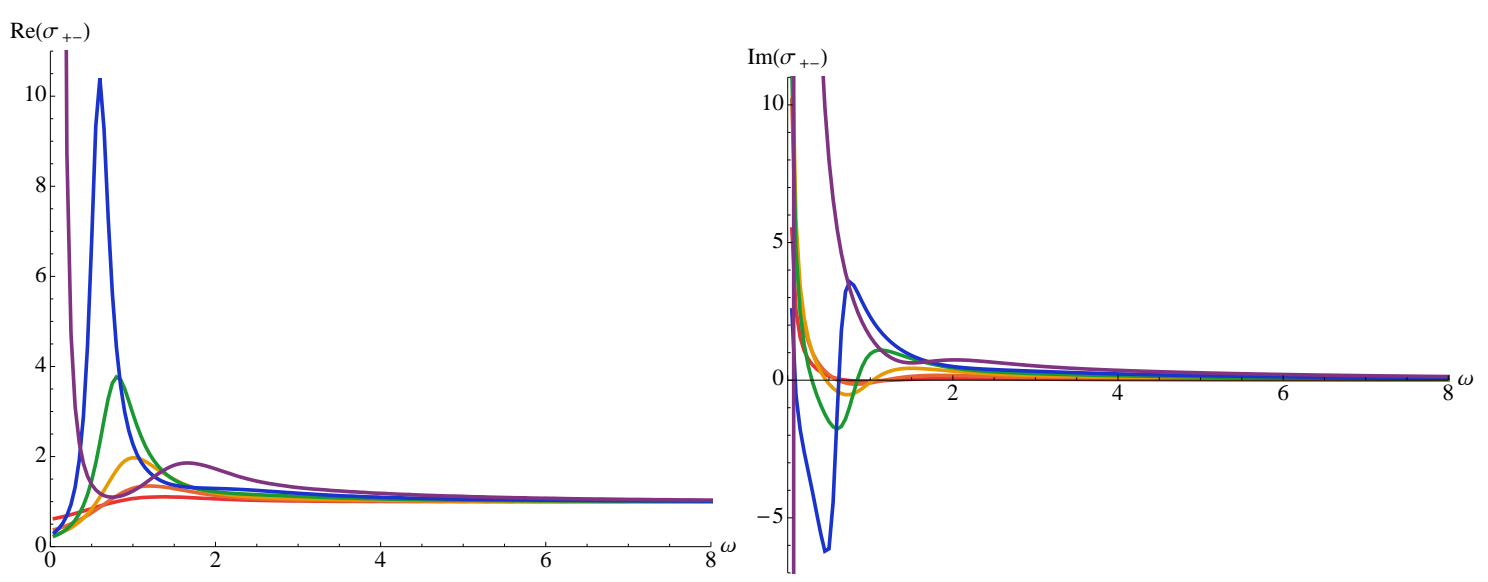

Figure 8.15: Real (left) and imaginary (right) part of the conductivity $\sigma_{+-}$for temperatures in the range $T / T_{c} \approx 0.91-0.41$, from red to purple.

\subsubsection{Quasinormal Modes}

Let us finally study the QNM spectrum in the (1) - (2) sector. This sector contains the fluctuations $\eta, a_{\mu}^{i}$ with $i=1,2$, therefore in the unbroken phase the spectrum will contain two diffusive modes associated with the two gauge fields. The fluctuations of the scalar field in the normal phase were already discussed in section 8.1. Analyzing the quasinormal mode spectrum in the broken phase amounts to solving the system of equations 8.2.70)-8.2.77). Details of the computation can be found in appendix 11.4

\section{Type II Goldstone mode}

As expected within the (1) - (2) sector we find a type II Goldstone mode. As in the ungauged model for small enough momentum its dispersion relation can be fitted to

$$
\omega= \pm \mathcal{B} k^{2}-i \mathcal{C} k^{2} .
$$

Figure 8.16 shows the dispersion relation for various values of the temperature in the hydrodynamic regime. The quadratic behavior with momentum is apparent. The temperature dependence of $\mathcal{B}$ and $\mathcal{C}$ is plotted in Figure 8.17. The value at $T=T_{c}$ is given by the same value as in the ungauged model 8.1 .33 and in fact can also be cross checked by calculating the scalar mode dispersion relation in the unbroken phase at $T=T_{c}$ since the QNMs must be continuous through the phase transition. We find a rather surprising dependence of $\mathcal{B}$ with the temperature. It starts at a finite value at the transition and then it rises rather sharply and falls off slower. It reaches a minimum at $T \approx 0.49 T_{c}$, temperature at which we found the change of sign in the residue of current-current correlators. We also find another peak around $T \approx 0.4 T_{c}$. We expect that it is again related with the instability found in the gauge sector around that temperature. It would also be interesting to calculate $\mathcal{B}(T)$ using an alternative method e.g. as the sound velocity can be calculated from thermodynamic considerations alone. In order to do this one would need to formulate the hydrodynamics of type II Goldstone modes. We are however not aware of such a hydrodynamic formulation and leave this for future research. The attenuation $\mathcal{C}(T)$ decreases rapidly with temperature. For temperatures $T / T_{c}<0.9$ it is negligible and the width of the type II Goldstone scales with $k^{4}$ in the hydrodynamic limit. This fast decreasing with temperature reflects that this mode propagates almost ideally in the fluid at low temperature. No further ungapped modes can be found in this sector. 


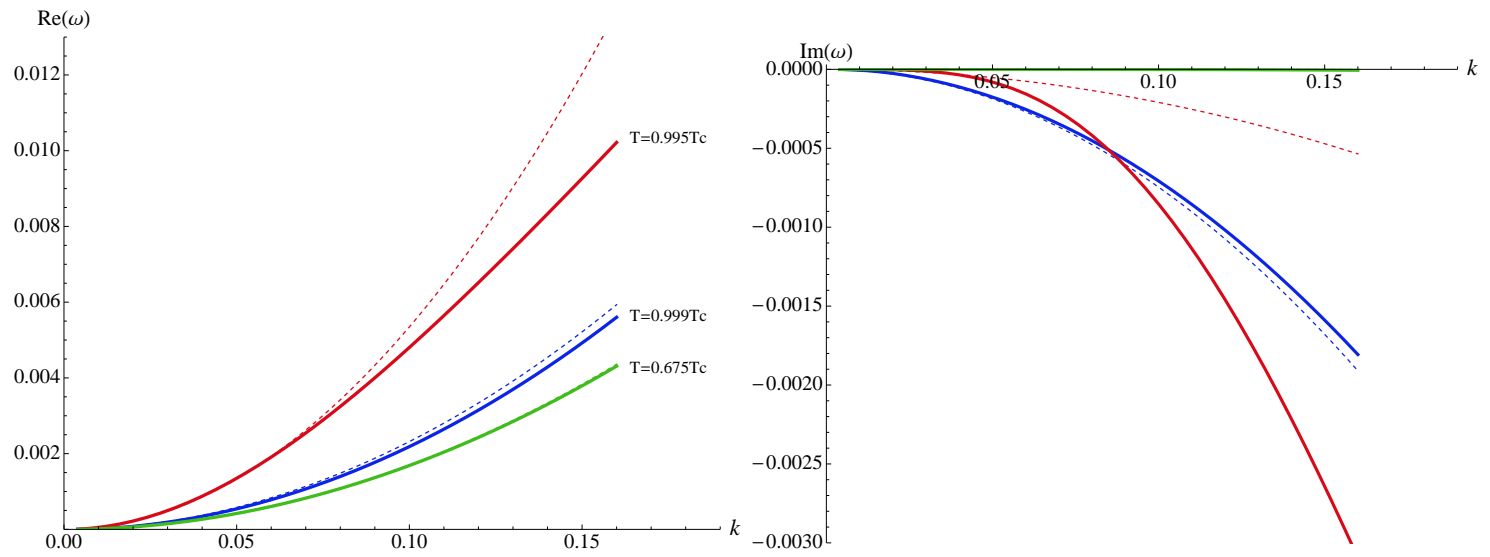

Figure 8.16: Plots of $\operatorname{Re}(\omega)$ (left) and $\operatorname{Im}(\omega)$ (right) as a function of the momentum. Thick lines correspond to data and thin lines to quadratic fit. At $T=0.995 T_{c}$ the real quadratic parameter $\mathcal{B}(T)$ shows a maximum, see Figure 8.17. Relation 8.2.92 is fulfilled with high accuracy.
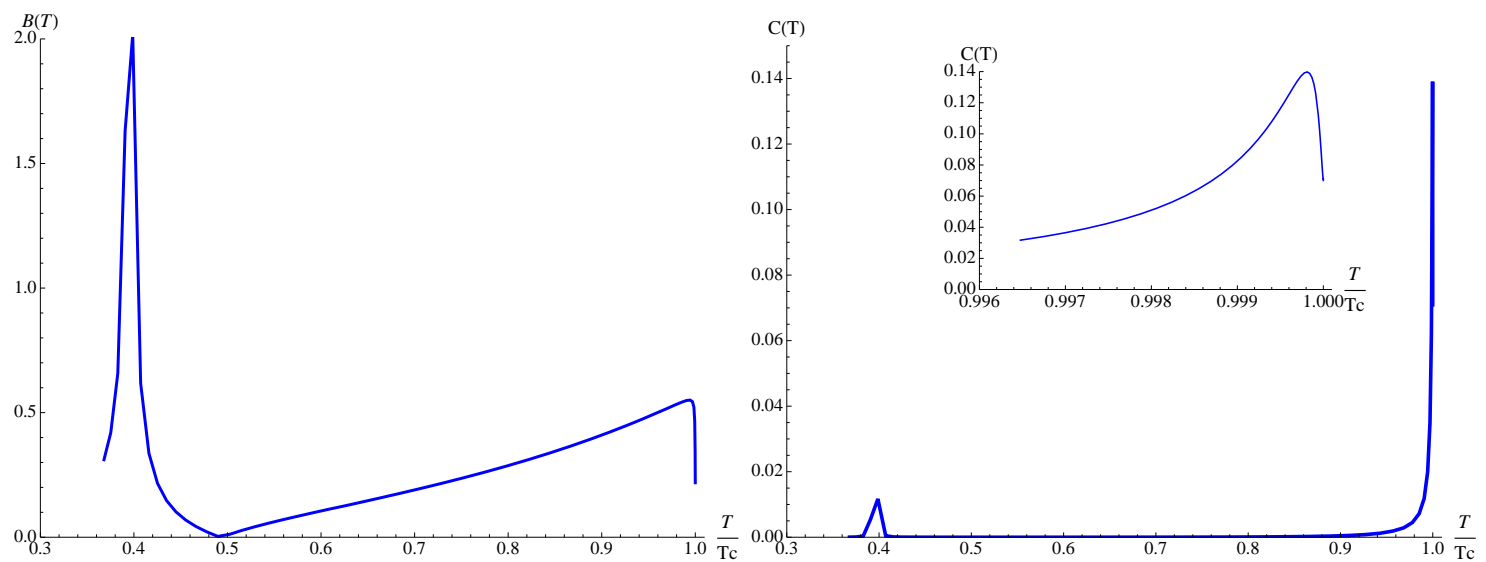

Figure 8.17: $\mathcal{B}$ (left) and $\mathcal{C}$ (right) as a function of $T / T_{c}$. The zoom-in shows the peak of $\mathcal{C}$ close to the transition. Furthermore at $T \simeq 0.4 T_{c}$ a sharp peak shows up in both coefficients. We relate this feature also to the instability arising in the vector sector. 


\section{Higher quasinormal modes}

Higher quasinormal modes correspond to gapped modes in the QNM spectrum and thus represent subleading contributions to the low energy Green's functions. We will focus here only on two of them: the continuation of the two diffusive modes of the unbroken phase and the special gapped mode that appears as the partner mode of the type II Goldstone mode in the field theoretical model.

Analyzing the first one is interesting in order to understand if also a qualitative change in the response pattern, such as that characterized by $T_{*}$ in the $U(1)$ superconductor sector, exists in the (1) - (2) sector. Since in this sector there exist however two diffusive modes in the unbroken phase it is also possible that the diffusive modes do not simply develop a gap but that they pair up and move off the imaginary axis in the broken phase. Indeed as we will see this is what happens.

The special gapped mode corresponds to a mode that is associated to the complex conjugate of the scalar perturbation in the unbroken phase. At $k=0$ and $\mu=0$ the scalar mode and its complex conjugate are degenerated. As we lower the temperature they split into two different modes. When we reach $T=T_{c}$, the lowest scalar mode becomes the type II Goldstone mode whereas the mode of the complex conjugate scalar field turns into the special gapped mode. The gap of this mode is expected to be given by the tree level result 4.4.30 [118].

Fate of diffusive modes: As already mentioned, in the (1) - (2) sector we have two degenerate diffusive modes in the unbroken phase. When going through the phase transition these modes can therefore pair up and move off the imaginary axes such that their quasinormal frequencies develop real parts and lie symmetrically around the imaginary axis. We expect therefore that in the low energy limit the dispersion relation takes the form

$$
\omega=\Gamma(T)+\mathcal{M}(T) k^{2},
$$

where both coefficients are complex functions and the second mode is located at $\omega^{\prime}=-\omega^{*}$. Besides, we expect the QNMs to be continuous through the phase transition, which in particular means that for $T=T_{c}$, our pseudo-diffusive modes should match the unbroken phase values, i.e. $\Gamma\left(T_{c}\right)=0$ and $\mathcal{M}\left(T_{c}\right)=-i$.

The modes at zero momentum are plotted in Figure 8.18. We see that indeed the gap vanishes as $T \rightarrow T_{c}$, whereas the modes split and develop a real part as we decrease the temperature. This last feature is exclusive of the non-Abelian system and thus does not take place in the usual $U(1)$ holographic superconductor, where the gap is purely imaginary (see [2] and comments above). Close to the phase transition, they present a linear behavior in temperature,

$$
\Gamma(T)=(4.1-0.8 i)\left(1-\frac{T}{T_{c}}\right) \quad \text { near } T_{c} .
$$

The temperature dependence of the coefficient of the momentum in 8.2.93, $\mathcal{M}(T)$, is shown in Figure 8.19. The real part rises very steeply just below the phase transition. The imaginary part approaches the unbroken phase value at the critical temperature, i.e. $\mathcal{M}\left(T_{c}\right)=-i$, as is expected for the pseudo-diffusion modes to continuously connect to the normal diffusion modes through the phase transition. Notice $\operatorname{Im} \mathcal{M}(T)$ decreases when lowering the temperature.

Another check of the fact that the pseudo diffusion modes come from the pairing up of the diffusion modes of the normal phase is that their dispersion relation at the phase 


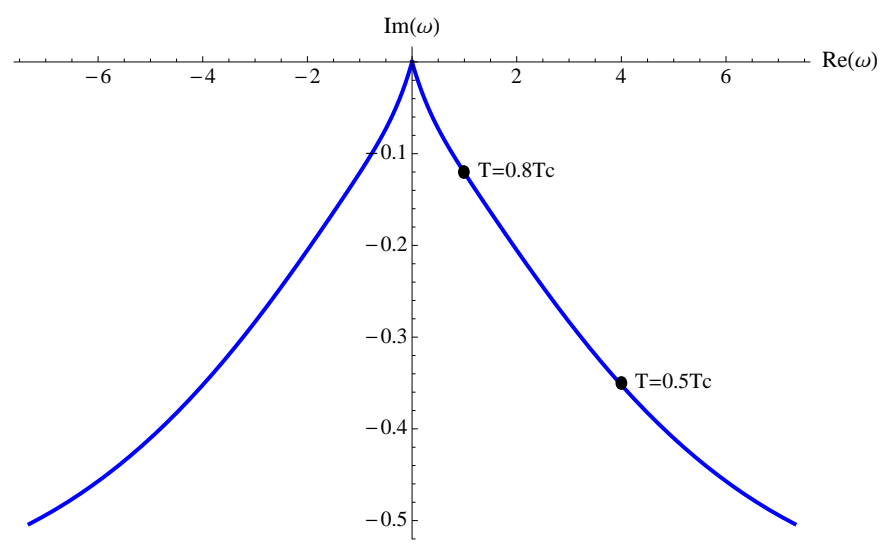

Figure 8.18: $\operatorname{Im} \omega$ versus $\operatorname{Re} \omega$ at $k=0$ as a function of the temperature. The shape of the figure is compatible with $T$ symmetry, since there are two pseudo-diffusive modes. Having $\operatorname{Re} \omega(k=0) \neq 0$ is characteristic of the non-Abelian case.

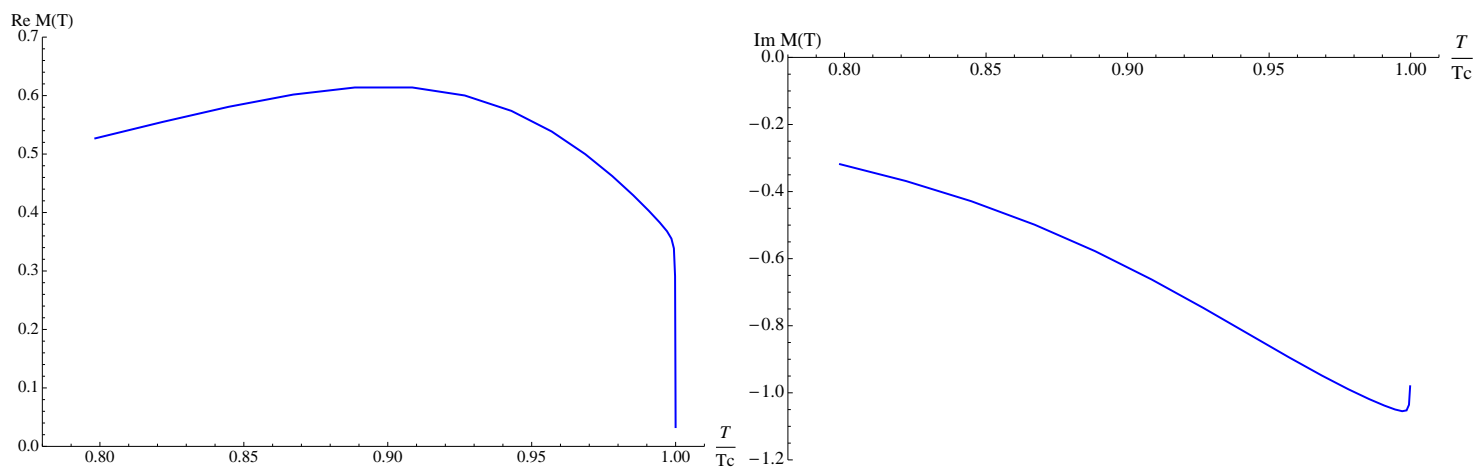

Figure 8.19: Real (left) and imaginary (right) part of $\mathcal{M}(T)$ as a function of $T / T_{c}$. As the temperature approaches $T_{c}$, the value of $\mathcal{M}(T)$ reaches the one prescribed by continuity through the phase transition. 
transition matches. Therefore the two diffusive modes are continuous through the transition, as expected for second order phase transitions, however instead of simply developing an imaginary gap to drop out of the hydrodynamic spectrum as for the usual $U(1)$ superconductor, they pair up in two modes that on top of this gap also develop a real part.

The fact that $\operatorname{Re}(\omega)$ does not vanish for these modes implies that sufficiently close to $T_{c}$ and in the limit $k=0$, the late-time response of the perturbed state will present an oscillatory decay of the perturbations, meaning that, contrary to the $U(1)$ case, there will not be a temperature at which the late-time behavior changes qualitatively.

Special Gapped mode: Seeking for this mode is computationally much more involved. Its behavior is characterized by a gap that is proportional to $\mu$. In particular, in [118 it was argued that a type II Goldstone mode is accompanied by a gapped mode obeying $\omega(0)=q \mu$ with $q$ being the charge of the corresponding field. In our conventions here we have $q=1$. So we have to look for a mode with $\omega(k=0)=\mu$. Furthermore we expect that it connects to the lowest mode of the complex conjugate scalar in the unbroken phase. In Figure 8.20 we depict such mode at zero momentum with respect to the chemical potential $\bar{\mu}$ in numerical units. Notice that the mode is continuous at the phase transition, as expected. We observe the linear behavior with the chemical potential that is predicted theoretically, at least near $\bar{\mu}_{c}$. It is very difficult to do the analysis when $\bar{\mu}>6$ due to the high computational power demanded to carry out the computation. The mode shows of course also a non-vanishing imaginary part which is due to the dissipation at finite temperature. We find that the real part above the phase transition can be approximated by

$$
\operatorname{Re} \omega=1.10 \bar{\mu} \quad \text { near } \bar{\mu}_{c} .
$$

This result shows a deviation from the conjectured behavior which could nevertheless be due to uncertainties in the numerics. Let us emphasize here that the numerics involved in tracking this mode through the phase transition were rather challenging.

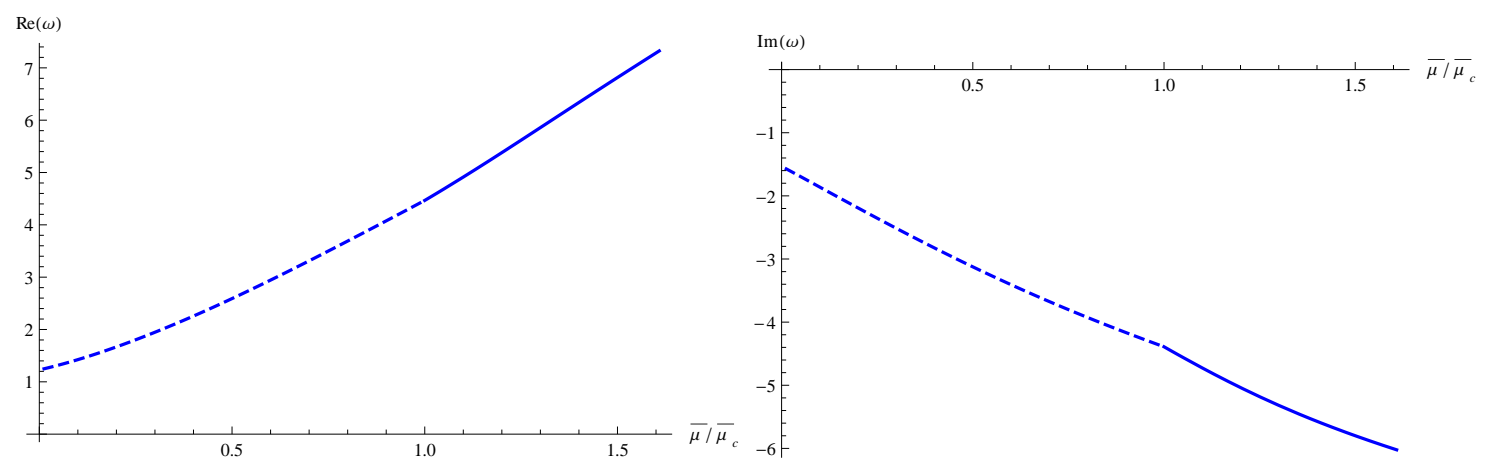

Figure 8.20: Real (left) and imaginary (right) part of the special gapped mode versus the chemical potential. We encounter the expected linear behavior with $\mu$. The plot covers both the unbroken (dashed line) and the broken (solid line) phases. 


\section{Chapter 9}

\section{The Landau criterion in Holography}

The characteristic property of a superfluid is its ability to flow totally frictionless through thin capillaries. From out introduction of Section ?? it is clear that one can think of a superfluid as a two component liquid. One component is the ground state with a macroscopic occupation number and the other is the normal component, subject to friction and viscosity. At very low temperatures the normal component can be described as the gas of elementary quasi particle excitations above the macroscopically occupied ground state. As indicated in the introduction, a famous argument due to Landau 93, 157, 158 sets a limit to the flow velocity that the condensate can obtain. Recall that it states that at zero temperature there is a critical flow velocity above which the superfluid ceases to exist

$$
v_{\max }=\min \frac{\epsilon(\vec{k})}{k},
$$

where the minimum over all elementary excitation branches has to be taken. The quantity $\epsilon(\vec{k})$ is the energy of the quasiparticle in the rest frame of the condensate. It is known for example for superfluid helium that the low temperature normal component can be well described as a gas of phonons and rotons and that the critical velocity is not determined by the minimum of the phonon and roton dispersion relation but rather by the excitation of vortices, resulting in a much lower critical velocity.

At higher temperatures there is always a normal component present and therefore the energy of an excitation of a superfluid with superflow can not be obtained by a (Galilean) boost $\epsilon^{\prime}(\vec{p})=\epsilon(\vec{p})+\vec{v} \cdot \vec{p}$. It is however still true that the energy will depend on the superfluid velocity and that it can become negative if the superfluid velocity is too large. At finite temperature the criterion is therefore that the superflow is stable as long as the energy of all quasiparticle excitations is positive. If in a superfluid the only low energy excitations are the phonons that criterion is basically the statement that the superflow dependent sound velocity is positive for all directions.

Focusing on holography, in $[159,160$ an s-wave superfluid in $2+1$ dimensions with superflow was constructed and it was pointed out that there is indeed a critical velocity above which the superfluid state ceases to exist. The phase diagram obtained in these works was based on comparing the free energy of the superflow with the free energy of the normal phase. It turned out that the phase transition from the superfluid phase to the normal phase was either first or second order depending on the temperature. Remarkably enough, in $3+1$ dimensions there is some range of masses of the condensate for which the 
phase transition is always of second order type [161. Another way of establishing the phase diagram has been used in [162]. There the supercurrent was fixed and it was argued that the phase transition is always first order.

The physical significance of the comparison of the free energies of the state with superflow and the normal state is not totally clear, since for all temperatures below the critical temperature the normal state is unstable towards condensation to the superfluid state without superflow. Indeed the superflow by itself is a metastable state only [158] as emphasized already in 159 . We will propose a different method of characterizing the phase diagram more directly related to the stability criterion (9.0.1).

The purpose of this chapter is thus to revisit the question of the realization of the stability criterion (9.0.1) in holographic superfluids. We will investigate the stability of the superflow via a QNM analysis of the $U(2)$ model (equation (8.2.34)). As already noticed, this automatically will give new and valuable information about the usual $U(1)$ holographic superfluid since a subsector of the linear fluctuations in the $U(2)$ model is isomorphic to it.

With this aim, we will follow 159,160 and reproduce the phase diagram based on the comparison of the free energy of the superflow with the normal phase. Then we will study the QNM spectrum with the superflow. In particular we will calculate the direction dependent speed of sound. We will indeed find that as the superfluid velocity is increased the speed of sound in opposite direction to the superflow is diminished and eventually vanishes at a critical velocity $v_{c}$. Increasing the superfluid velocity even further this sound velocity becomes negative and this has to be interpreted as the appearance of a negative energy state in the spectrum. In principle that would be enough to argue for instability but at basically no price the QNM analysis can give us an even clearer sign of instability. It is well-known that the imaginary part of the QNMs have to lie all in the lower half plane. If they fail to do so an exponentially growing mode with amplitude $\phi \propto \exp (\Gamma t)$ appears in the spectrum. It is not necessary for this mode to have zero momentum. In fact we will see that if we increase the superfluid velocity beyond the critical value the imaginary part of the sound mode quasinormal frequency moves into the upper half plane. And it does so attaining a maximum for non-zero momentum. We will see that this behavior is necessary to connect the phase diagram continuously to the normal phase. Then moving slightly aside we will study the conductivities with superflow. This has been done before but only in the transverse sector and here we present results for the longitudinal sector.

Finally we will briefly investigate the fate of the type II Goldstone mode in the $U(2)$ model. We will study both the gauged and the ungauged model of the previous chapter. Landau's criterion suggests that these setups do not sustain any finite superflow since $\min \frac{\epsilon(k)}{k}=0$ for quadratic dispersion relations. Again we can not only look at the real part but also at the imaginary part. We will indeed find poles in the upper half plane for non-zero momenta for all temperatures and superfluid velocities for the gauged and the ungauged model 1 .

\footnotetext{
${ }^{1}$ Models with one $U(1)$ gauge field and two complex scalars similar to our ungauged model were studied before in 163 and recently in 164. (see also 165]). There the two scalars had however different masses and this should prevent the appearance of the ungapped type II Goldstone mode.
} 


\subsubsection{The $U(2)$ superfluid with superflow}

We will be working essentially with the same gauged $U(2)$ model presented before, i.e.

$$
S=\int d^{4} x \sqrt{-g} \mathcal{L}=\int d^{4} x \sqrt{-g}\left(-\frac{1}{4} F^{\mu \nu c} F_{\mu \nu}^{c}-m^{2} \psi^{\dagger} \psi-\left(D^{\mu} \psi\right)^{\dagger} D_{\mu} \psi\right),
$$

where

$$
\psi=\sqrt{2}\left(\begin{array}{c}
\lambda \\
\Psi
\end{array}\right), \quad A_{\mu}=A_{\mu}^{c} T_{c}, \quad D_{\mu}=\partial_{\mu}-i A_{\mu},
$$

where we include the $\sqrt{2}$ in the definition of the scalar field to agree with the equations of [159]. Following [144] we choose the mass of the scalar field to be $m^{2}=-2 / L^{2}$. We take the generators of $U(2)$ as in 8.2 .36 ). Since we will work in the probe approximation we do not include the metric in the dynamical degrees of freedom but simply consider (9.0.2) in the background metric of the Schwarzschild-AdS black brane of (8.1.3). By suitable rescalings we can set $L=r_{H}=1$ and work with dimensionless coordinates.

In order to find background solutions corresponding to a condensate with non-vanishing superfluid velocity we proceed as follows. First note that the scalar field $\lambda(r)$ can be set to zero by a $U(2)$ gauge transformation. For the scalar $\Psi$ we demand then that the nonnormalizable mode vanishes. By a residual $U(1)$ gauge transformation we can also take $\Psi$ to be real.

Now we need to define what we mean by the superflow. Let us discuss this for a moment from a field theory perspective. In a multi-component superfluid with $U(2)$ symmetry we can in principle construct the four (super) currents

$$
J_{a}^{\mu}=\Phi^{\dagger} T_{a} \nabla^{\mu} \Phi-\left(\nabla^{\mu} \Phi\right)^{\dagger} T_{a} \Phi,
$$

where $\nabla^{\mu}=\partial^{\mu}-i A_{a}^{\mu} T_{a}$ is the covariant derivative and $\Phi$ is the condensate wave function which transforms as a doublet under $U(2)$. If the condensate is such that one of the spatial currents does not vanish we can speak of a state with non-vanishing superflow. By a gauge transformation we can always assume the condensate to take some standard form, e.g. $\Phi=(0, \phi)^{T}$ and represent the non-vanishing superflow in terms of constant gauge fields. Since we are interested in the case where we break the $U(2)$ symmetry spontaneously to $U(1)$ we will only allow a non-zero gauge field in the overall $U(1)$ corresponding to the generator $T_{0}$. Furthermore by an $S O(2)$ rotation we can take the gauge field to point into the $x$ direction. From 9.0 .4 it is easy to see that such a superflow has non-vanishing currents $J_{x}^{(0)}$ and $J_{x}^{(3)}$. In order to find solutions with non-trivial charge we also need to introduce a chemical potential. Again in order to preserve the full $U(2)$ symmetry we also allow a chemical potential only for the overall $U(1)$ charge.

Returning now to Holography these considerations determine the ansatz for the gauge fields to be of the form

$$
A^{(0)}=A_{t}^{(0)}(r) d t+A_{x}^{(0)}(r) d x, \quad A^{(3)}=A_{t}^{(3)}(r) d t+A_{x}^{(3)}(r) d x .
$$

While we introduce sources only for $A^{(0)}$ the fact that also the current $J_{\mu}^{(3)}$ is nonvanishing demands that $A^{(3)} \neq 0$. The physical interpretation for this fact is that the system forces the appearance of a charge density $\rho^{(3)} \neq 0$ (as pointed out in Chapter 8 ) and a current $J_{x}^{(3)}$ in the vacuum with superflow. This is in turn closely related to the presence of type II Goldstone bosons in the spectrum [112]. 
At this point it is important to note that the above identification is only valid in the superfluid phase, that is, whenever $\Psi \neq 0$. A constant background value of the gauge field $A_{x}$ in the normal phase is not physically meaningful since there is no notion of superflow.

For the reasons outlined above we choose the asymptotic boundary conditions for the gauge fields to be

$$
\begin{array}{ll}
A_{t}^{(0)}(r \rightarrow \infty)=2 \bar{\mu}, & A_{t}^{(3)}(r \rightarrow \infty)=0, \\
A_{x}^{(0)}(r \rightarrow \infty)=2 \bar{S}_{x}, & A_{x}^{(3)}(r \rightarrow \infty)=0 .
\end{array}
$$

Recall that $\bar{\mu}$ is to be identified with the chemical potential of the dual theory and $\bar{S}_{x}$ is related to the superflow velocity. We have included a factor of two in the definitions of $\bar{\mu}$ and $\bar{S}_{x}$ for the following reason. The background field equations can be recast in the form of those derived from the $U(1)$ model in $[159,160]$ by using the field redefinitions

$$
\begin{aligned}
A_{0} & =\frac{1}{2}\left(A_{t}^{(0)}-A_{t}^{(3)}\right), & \xi & =\frac{1}{2}\left(A_{t}^{(0)}+A_{t}^{(3)}\right), \\
A_{x} & =\frac{1}{2}\left(A_{x}^{(0)}-A_{x}^{(3)}\right), & \varsigma & =\frac{1}{2}\left(A_{x}^{(0)}+A_{x}^{(3)}\right),
\end{aligned}
$$

for which the background equations now read

$$
\begin{aligned}
\Psi^{\prime \prime}+\left(\frac{f^{\prime}}{f}+\frac{2}{r}\right) \Psi^{\prime}+\left(\frac{A_{0}^{2}}{f^{2}}-\frac{A_{x}^{2}}{r^{2} f}-\frac{m^{2}}{f}\right) \Psi & =0, \\
A_{0}^{\prime \prime}+\frac{2}{r} A_{0}^{\prime}-\frac{2 \Psi^{2}}{f} A_{0} & =0, \\
A_{x}^{\prime \prime}+\frac{f^{\prime}}{f} A_{x}^{\prime}-A_{x} \frac{2 \Psi^{2}}{f} & =0, \\
\xi^{\prime \prime}+\frac{2}{r} \xi^{\prime} & =0, \\
\varsigma^{\prime \prime}+\frac{f^{\prime}}{f} \varsigma^{\prime} & =0 .
\end{aligned}
$$

It can be checked that we recover the usual $U(1)$ system describing the $U(1)$ holographic superconductor in the presence of superfluid velocity (see for instance 161). The chemical potential $\bar{\mu}$ is therefore the chemical potential for the field $A_{0}$ which plays the role of the temporal component of the (single) gauge field, and $A_{x}$ plays the role of the spatial component of the single gauge field of 159 161]. This explicitly shows that the background of the $U(2)$ model is identical to that of the $U(1)$ superconductor, even for a nonzero superfluid velocity.

An immediate consequence of the fact that the background equations are those of the $U(1)$ holographic superfluid is that, at first sight, the $U(2)$ system seems to be able to accommodate a superflow. However, as already argued, this is in direct contradiction with the Landau criterion of superfluidity [158] due to the presence of a type II Goldstone in the spectrum. Of course, having found solutions to the equations of motion does not yet say anything about the stability. In fact as we will explicitly see the type II Goldstone will turn into an unstable mode and therefore make the whole $U(2)$ solution with superflow unstable.

Equations (9.0.8)-(9.0.10) are non-linear and have to be solved using numerical methods. Notice that (9.0.11) and $(9.0 .12)$ are decoupled. They correspond to the preserved $U(1)$ symmetry after having broken spontaneously $U(2) \rightarrow U(1)$. The asymptotic behavior 
of the fields close to the conformal boundary is

$$
\begin{aligned}
& A_{0}=\bar{\mu}-\frac{\bar{\rho}}{r}+\ldots, \\
& A_{x}=\bar{S}_{x}-\frac{\overline{\mathcal{J}}_{x}}{r}+\ldots, \\
& \Psi=\frac{\psi_{1}}{r}+\frac{\psi_{2}}{r^{2}}+\ldots
\end{aligned}
$$

The asymptotic quantities are related to the physical ones by

$$
\begin{array}{ll}
\bar{\mu}=\frac{3}{4 \pi T} \mu, & \bar{\rho}=\frac{9}{16 \pi^{2} T^{2}} \rho, \\
\bar{S}_{x}=\frac{3}{4 \pi T} S_{x}, & \overline{\mathcal{J}}_{x}=\frac{9}{16 \pi^{2} T^{2}} \mathcal{J}_{x}, \\
\psi_{1}=\frac{3}{4 \pi T}\left\langle\mathcal{O}_{1}\right\rangle, & \psi_{2}=\frac{9}{16 \pi^{2} T^{2}}\left\langle\mathcal{O}_{2}\right\rangle .
\end{array}
$$

We are working in the grand canonical ensemble, then we fix the chemical potential $\mu$. The temperature is defined by $T / \mu \propto 1 / \bar{\mu}$. For studying the evolution of the condensate as a function of the superfluid velocity, the natural way to proceed is to work with $S_{x} / \mu$ as our free parameter together with temperature. Notice that both asymptotic modes of the scalar field are actually normalizable 150 . From now on we will stick to the $\mathcal{O}_{2}$ theory, for which $\psi_{1}=0$ and $\left\langle\mathcal{O}_{2}\right\rangle$ is the vev of a scalar operator of mass dimension two in the dual field theory. Notice that the fields $\xi$ and $\zeta$ corresponding to the unbroken $U(1)$ are given by

$$
\begin{aligned}
& \xi=\bar{\mu}-\bar{\rho} / r, \\
& \zeta=\bar{S}_{x},
\end{aligned}
$$

even with non-vanishing condensate. The values of the condensate as a function of temperature and superfluid velocity shown in Figure 9.1 reproduce the previous results of [159, 160]. In the plot and in the rest of the section the temperature is measured with respect to the critical temperature of the phase transition with no superfluid velocity, i.e. $T_{c} \approx 0.0587 \mu$.

\subsubsection{Free Energy}

In this section we compute the free energy of the condensed phase and compare it to the free energy of the unbroken phase as done in 159,160. After appropriate renormalization of the Euclidean on-shell action and using the boundary conditions (9.0.13), the free energy density reads

$$
F=-T S_{r e n}=-\bar{\mu} \bar{\rho}+\bar{S}_{x} \overline{\mathcal{J}}_{x}+\int_{1}^{\infty} d r\left(\frac{2 r^{2} A_{0}^{2}}{f}-2 A_{x}^{2}\right) \Psi^{2} .
$$

In the normal phase $\Psi=0$, regularity at the horizon forces the $A_{x}$ gauge field to have a trivial profile along the radial direction in the bulk and therefore not to contribute to the free energy, i.e. $\overline{\mathcal{J}}_{x}=0$. This is in accordance with the fact that in absence of a scalar condensate it is not possible to switch on a superfluid velocity anymore. Switching on the spatial component of the gauge field in the normal phase describes a pure gauge transformation that does not affect the free energy of the system. In the broken phase instead, different superfluid velocities are physically distinguishable. It is important to 


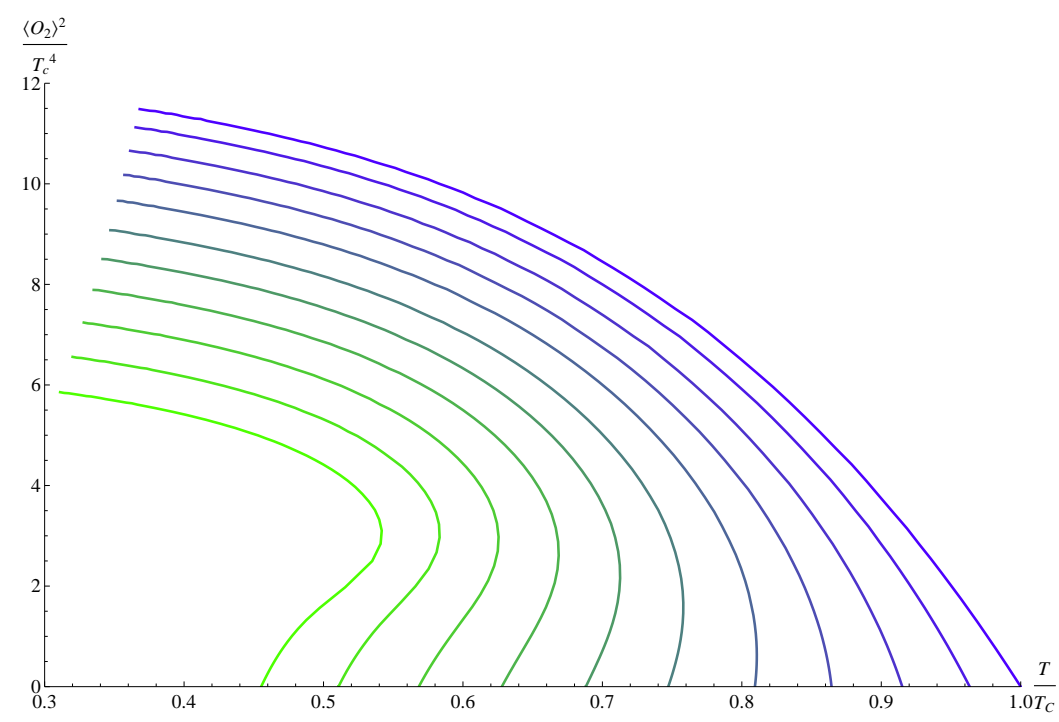

Figure 9.1: The condensate for different values of the superfluid velocity, ranging from $\frac{S_{x}}{\mu}=0.005$ (right) to $\frac{S_{x}}{\mu}=0.530$ (left).

emphasize that one is actually comparing the normal phase at vanishing superfluid velocity with the superconducting phase at different values of the superfluid velocity, and that the normal phase is unstable towards condensation without superflow for any $T<T_{c}$. Therefore, the physical relevance of this comparison is not completely clear. We will see later on that actually the Landau criterion establishes a different transition temperature for the superfluid phase. Nevertheless the free energy gives a natural first approach to characterize the phase diagram of the system. We would like to remark that the superflow phase is just a metastable phase, since the true background is the static condensed phase which allways has lower free energy [158, [159].
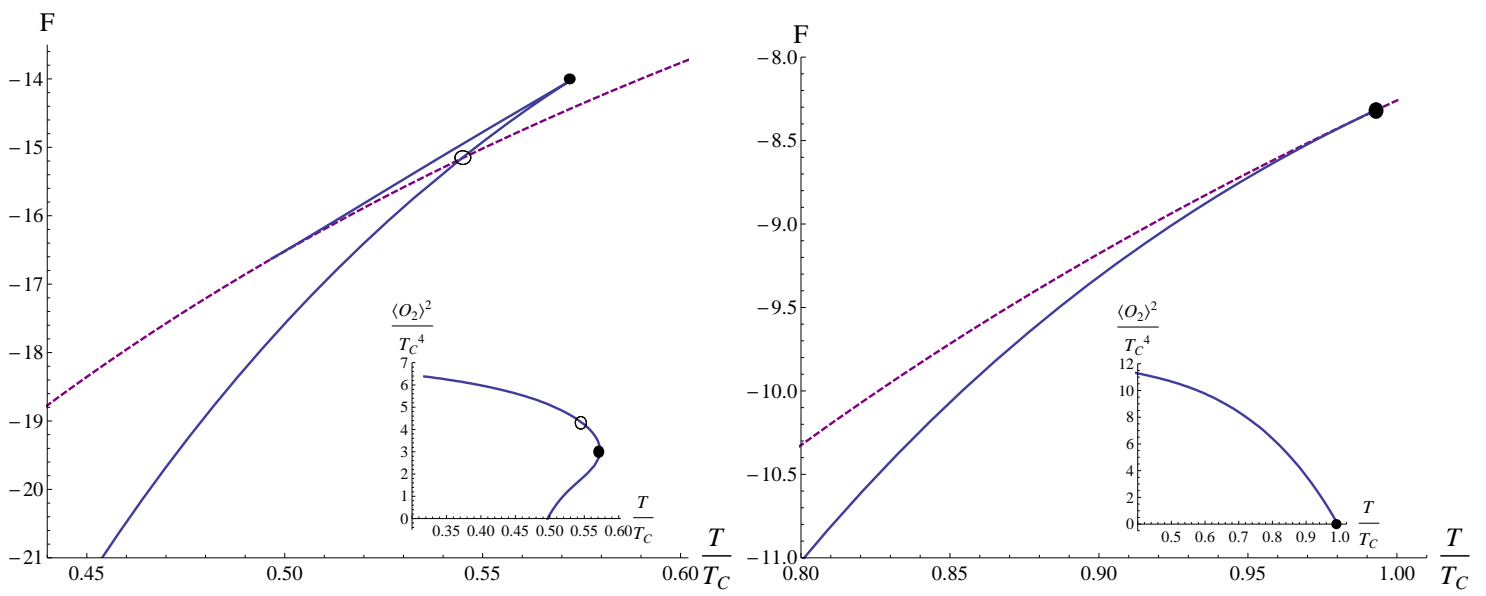

Figure 9.2: Free energy of the condensed (solid line) and normal (dashed line) phases for $\frac{S_{x}}{\mu}=0.5$ (left) and $\frac{S_{x}}{\mu}=0.05$ (right). The small plots show the behavior of the condensate. The open circle corresponds to the critical temperature $\tilde{T}$ whereas the filled circle corresponds to the spinodal point (max. overheating).

In Figure 9.2 the free energy of both the normal and condensate phase is plotted for different values of $\frac{S x}{\mu}$. The different behavior for large and small values of the superfluid 
velocity is apparent. For large superfluid velocity the transition is first order as can be seen from the left panel in Figure 9.2 , indicated by the open circle. Coming from low temperatures the system can still be overheated into a metastable state until the point of spinodal decomposition where the order parameter susceptibility $\partial\langle\mathcal{O}\rangle / \partial \mu$ diverges, indicated by the filled circle.

For low superfluid velocities the normal phase free energy and the condensate free energy match smoothly at a second order phase transition. The resulting phase space is contained in Figure 9.6 and reproduces the previous analysis in [159, 160].

The phase transition found from considerations of the free energy is however only apparent. We will call the temperature at which the free energies of the condensate phase with superflow and the free energy of the normal phase coincide $\tilde{T}$ from now on. The temperature at which the (second order) phase transition occurs without superflow we will denote by $T_{c}$. As we will show now the superflow becomes unstable at temperatures below $\tilde{T}$ as implied by the Landau criterion applied to the sound mode. This temperature we will denote by $T^{*}$.

\subsubsection{Landau criterion for the $U(1)$ sector}

In this section we analyze the QNM spectrum of the (0) - (3) sector, which is identical to the original $U(1)$ holographic superconductor in the presence of superfluid velocity 159 160]. We focus on the behavior of the lowest QNM, the type I Goldstone boson, with special emphasis on the velocity and the attenuation constant and their dependence on the superfluid velocity and on the angle of propagation with respect to the flow.

To study the QNM spectrum we consider linearized perturbations around the background of the fields of the form $\delta \phi_{I}=\delta \phi_{I}(r) \exp [-i(\omega t-|k| x \cos (\gamma)-|k| y \sin (\gamma)]$. Specifically we consider the fluctuations

$$
\begin{aligned}
\delta \hat{\Psi}^{T} & =(\eta(r), \sigma(r)), \\
\delta A^{(0)} & =a_{t}^{(0)}(r) d t+a_{x}^{(0)}(r) d x+a_{y}^{(0)}(r) d y, \\
\delta A^{(3)} & =a_{t}^{(3)}(r) d t+a_{x}^{(3)}(r) d x+a_{y}^{(3)}(r) d y,
\end{aligned}
$$

where in the case of the gauge fluctuations we will work with the linear combinations already defined by 9.0 .7 , i.e. $a_{\mu}^{(-)} \equiv \frac{1}{2}\left(a_{\mu}^{(0)}-a_{\mu}^{(3)}\right)$ and $a_{\mu}^{(+)} \equiv \frac{1}{2}\left(a_{\mu}^{(0)}+a_{\mu}^{(3)}\right)$. The linearized equations are rather complicated and we list them in Appendix 11.1. The numerical techniques used to obtain the hydrodynamic modes in coupled systems are well known 2, 51] (see also Appendix 11.4).

In Figures 9.3 and 9.4 we represent the velocity and the attenuation of the type I Goldstone mode. Its dispersion relation is given by 8.1 .29 at low momentum, except now the speed of sound $v_{s}$ and the attenuation constant $\Gamma$ depend on the angle $\gamma 2$. Figure 9.3 shows the angle dependent variation of the sound velocity and damping constant for a fixed temperature and varying values of the superfluid velocity. Figure 9.4 shows the same at fixed superfluid velocity but with varying temperature. As one would expect for small $S_{x} / \mu$ and low enough temperature the velocity and damping constant are almost isotropic. As the superfluid velocity is increased or the temperature is increased the plot becomes more and more asymmetric. The anisotropy of the system is such that we see an enhancement of the sound velocity and a reduction of the damping in the direction of the superflow.

\footnotetext{
${ }^{2}$ The small real constant $b$ does not play a role here since for small enough momentum the linear part proportional to $v_{s}$ dominates.
} 


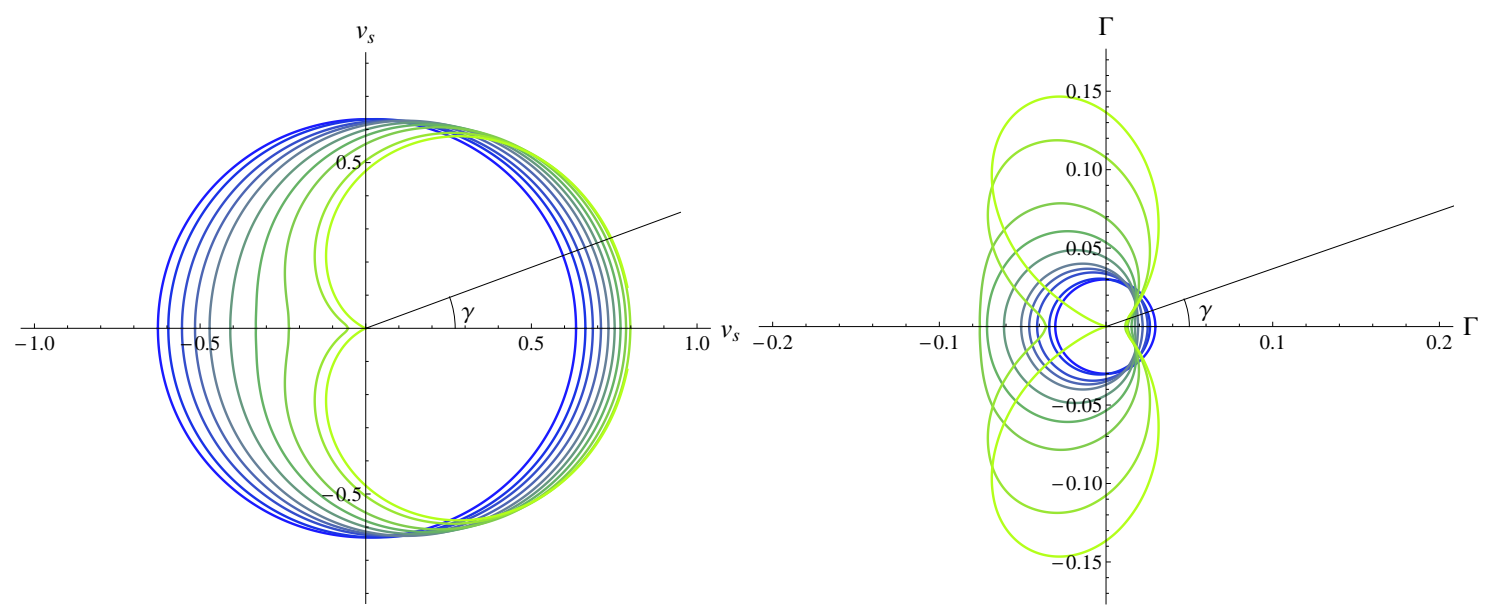

Figure 9.3: Sound velocity and damping for $T=0.7 T_{c}$ and several superfluid velocities from $S_{x} / \mu=0$ (blue) to $S_{x} / \mu=0.325$ (green). The radius represents the absolute value of the sound velocity (left) and attenuation constant (right) as a function of the angle $\gamma$ between the momentum and the superfluid velocity.

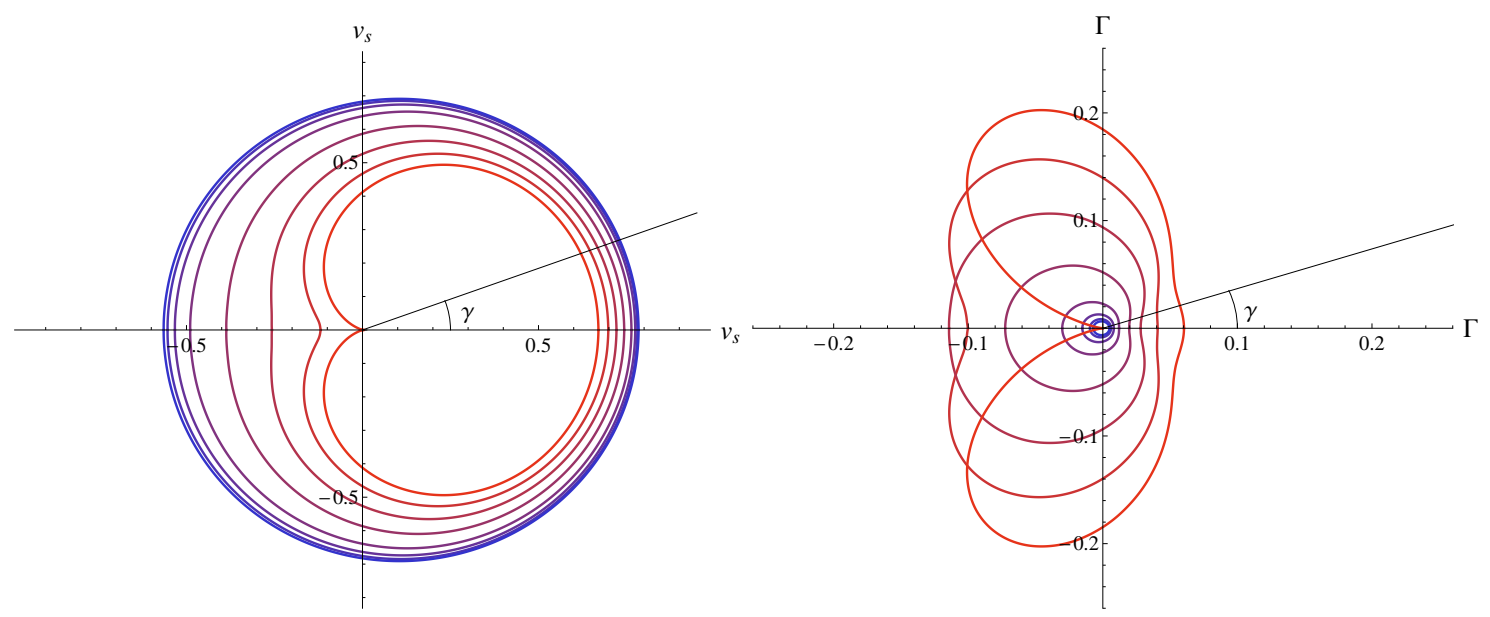

Figure 9.4: Sound velocity (left) and attenuation constant (right) for $S_{x} / \mu=0.2$ as a function of the angle $\gamma$ and for a range of temperatures from $T=0.85 T_{c}$ (red) to $T=0.57 T_{c}$ (blue).

The most interesting feature of the system is found however in the opposite direction to the superfluid velocity. As one can see in both plots, at $\gamma=\pi$ the reduction in the sound velocity is strongest and eventually both the attenuation constant and the sound velocity vanish simultaneously. It is important to stress that this happens below the temperature $\tilde{T}$. If one continues increasing the temperature (or equivalently increasing the superfluid velocity at fixed temperature) one finds that the real part of the frequency becomes negative and that its imaginary part crosses to the upper half plane, as depicted in Figure 9.5. This signals the appearance of a tachyonic mode. $T^{*}$ is the temperature where both the instability appears and the speed of sound becomes negative. This temperature actually signals the end of the superfluid phase according to the Landau criterion, and therefore we interpret it as the physical phase transition temperature.

In Figure 9.6 (left) we present the phase diagram resulting from the QNM analysis. 


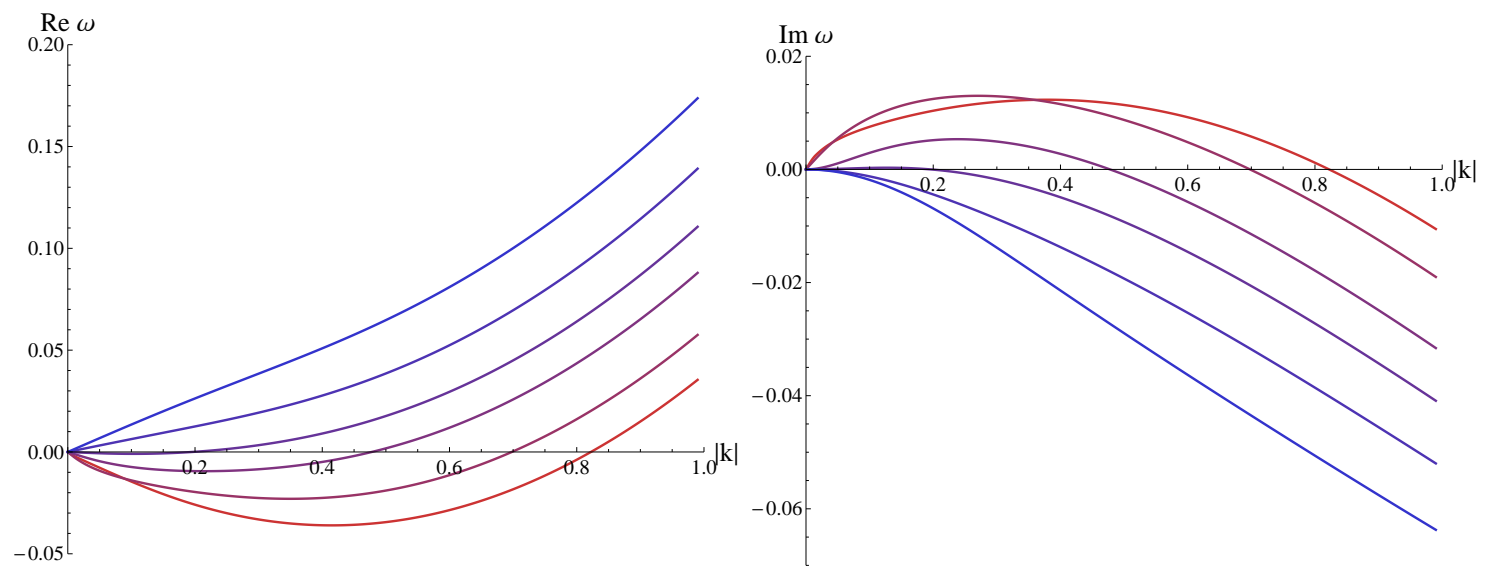

Figure 9.5: Real (left) and imaginary (right) parts of the frequency of the lowest hydrodynamic mode (type I Goldstone mode) versus momentum at $S_{x} / \mu=0.1$ and $\gamma=\pi$ for different temperatures from $T=\tilde{T}=0.970 T_{c}$ (red) to $T=0.905 T c$ (blue). The instability appears at $T^{*}=0.935 T_{c}$.

To illustrate the situation, on the right plot we show the behavior of the relevant QNM ${ }^{3}$ at three different points of the phase diagram 4 (points labelled 1, 2, 3 on the left plot). At $\tilde{T}<T<T_{c}$ in the unbroken phase (line $3_{N}$ ), the mode that was responsible for the transition to the homogeneous superfluid phase without superfluid velocity is shifted and becomes unstable at finite momentum. This behavior reflects the fact that the system is unstable for $T \leq T_{c}$, the mode being shifted in momentum due to the constant nonzero value of $A_{x}$. At $T=\tilde{T}$ (lines $2_{N, S}$ ) the lowest mode becomes unstable at $k=0$. It is at this point that the free energy of the homogeneous superfluid phase equals that of the normal phase. Hence, the free energy analysis, which only captures the $k=0$ dynamics, predicts a phase transition at this temperature. For the particular superfluid velocity in the plot the phase transition is second order. Finally, the fate of the QNM for $T^{*}<T<\tilde{T}$ is shown in lines $1_{N}$ (for the normal phase) and $1_{S}$ (for the homogeneous superflow phase). One can see that the Goldstone mode in the superfluid phase is unstable for a finite range in momentum. Only at $T^{*}$ this mode becomes stable again as shown in Figure 9.5. It is at this temperature that the homogeneous superflow phase becomes stable according to the Landau criterion since the sound velocity becomes positive (moreover the imaginary part of the QNM dispersion relation lies entirely in the lower half plane).

Therefore the QNM results indicate that a phase transition occurs at a lower temperature $T^{*}<\tilde{T}$. Similarly, if we imagine the system at fixed temperature and start rising the superfluid velocity, both $v_{s}$ and $\Gamma$ will vanish at some value of $S_{x} / \mu$, which we claim is indeed the critical velocity $v_{c}$ of the superfluid, in the sense of the Landau criterion.

As a very interesting fact, notice that the imaginary part of the mode exhibiting the instability has a maximum at finite momentum as well. The fact that the instability appears at finite momentum suggests that there might exist a new (meta)stable intermediate phase above $T^{*}$ with a spatially modulated condensate. Examples of such instabilities towards spatial modulation have been discussed before in $167-169$.

It is important to remark that, as shown in Figure 9.6 (right), for temperatures $T^{*}<$

\footnotetext{
${ }^{3}$ In the unbroken phase this is just the lowest scalar QNM, while in the broken phase it is the sound mode at fixed $S_{x} / \mu$.

${ }^{4} \mathrm{An}$ analogous discussion and phase space was found at weak coupling in 166 .
} 


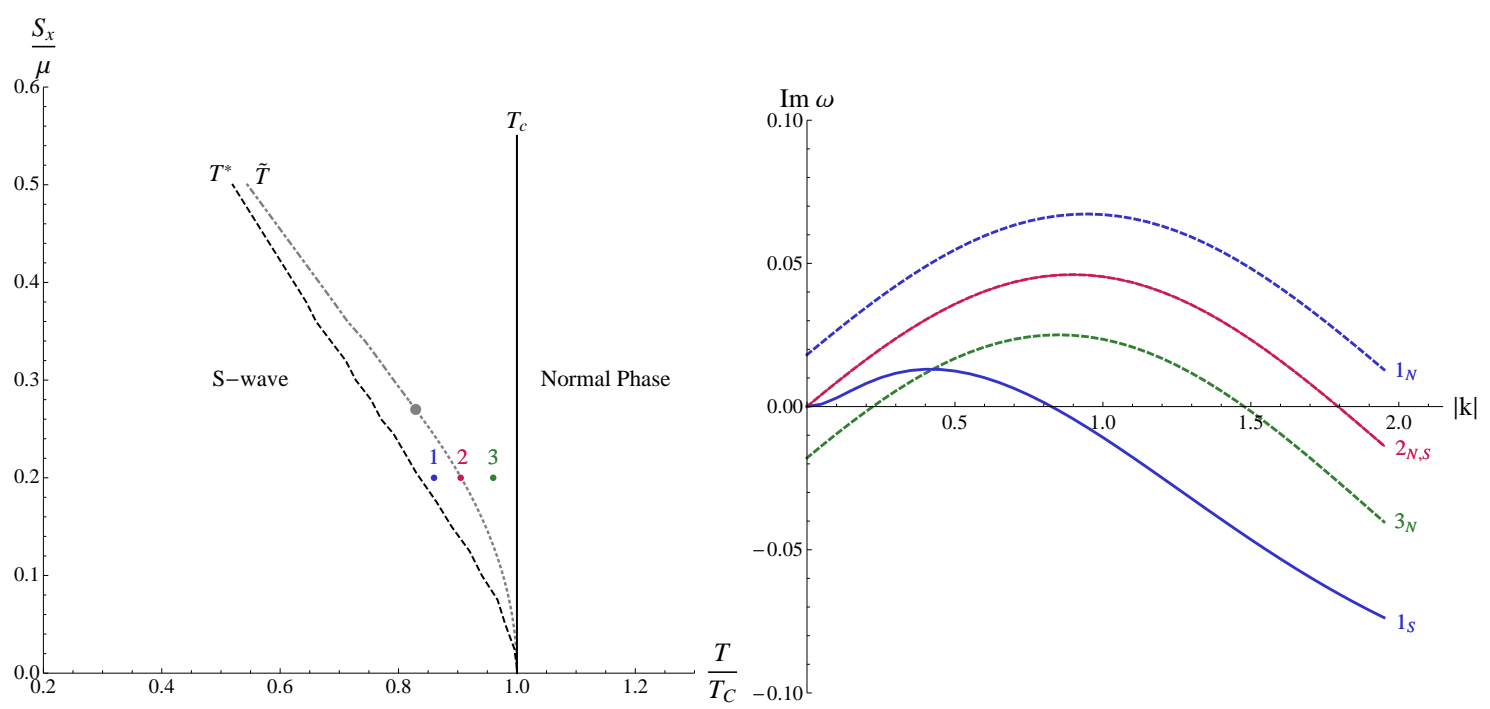

Figure 9.6: (Left) Phase diagram after the study of the QNMs . The grey dashed line corresponds to $\tilde{T}$, the apparent transition temperature found by direct analysis of the free energy. At a certain point (disk) the transition in free energy changes from 2nd order (dotted) to 1st order (dash-dotted). The black solid line corresponds to the critical temperature in absence of superfluid velocity. The black dashed line signals the physical phase transition at $T^{*}$, the temperature at which the local instability appears. Points 1, 2 and 3 indicate the values of temperature and velocity used in the plot on the right. (Right) Imaginary part of the lowest QNM for different temperatures at fixed $S_{x} / \mu=0.2$ and $\gamma=\pi$. Dashed lines were obtained in the normal phase whereas solid lines were calculated in the superfluid phase.

$T<\tilde{T}$ the mode responsible for the transition to the (shifted) homogeneous stationary phase (line $1_{N}$ ) and the new unstable mode (line $1_{S}$ ) show maxima at different momenta. We take this as an indication for existence of a new metastable in- homogeneous phase. The wave number of the modulation in this phase should be determined by the maximum of the line $1_{S}$.

Recall that the Landau criterion is formulated uniquely in terms of $\operatorname{Re}(\omega)$. At a given temperature the critical velocity corresponds to the superfluid velocity at which $v_{s}=0$, or equivalently to the value of $S_{x} / \mu$ where $\operatorname{Re}(\omega)$ becomes negative (see Figure 9.5). That the criterion is a statement about $\operatorname{Re}(\omega)$ reflects the fact that it holds also at zero temperature. At finite temperature the dispersion relation of the gapless mode gets itself altered due to both the superfluid velocity and the temperature [100, 158], implying that generically the critical value of $S_{x} / \mu$ at fixed temperature does not correspond to the velocity of sound at the same temperature and vanishing superfluid velocity.

An extra comment is in order here regarding the phase of the system for $T_{c}>T>\tilde{T}$. The fact that in the unbroken phase the lowest QNM is unstable in this regime (see line $3_{N}$ in Figure 9.6) of course indicates that the normal phase is unstable. Let us comment on this. Since the condensate vanishes in the normal phase, there exists no physical notion of superfluid velocity in this phase; different choices of $A_{x}$ are just different frame choices. In particular, a constant $A_{x}$ simply acts as a shift in momentum in the unbroken phase, as can be seen from the fact that the maximum of the QNM is centered at a momentum equal to the value of the gauge field at the conformal boundary. Therefore the normal phase is unstable for any temperature lower than the critical temperature $T_{c}$ towards the formation 
of a superfluid without superflow. On the other hand, we know that the homogeneous condensate solution with finite velocity does not exist in this region, and moreover it is unstable for $T>T^{*}$. We see two possibilities for the completion of the phase diagram in this region. First, the system could simply fall down to the true ground state, which is the condensate with no superflow. At finite $S_{x} / \mu$ this is still a solution which minimizes the energy albeit with a condensate that is not real anymore but rather has a space dependent phase such that $\vec{\nabla} \Phi=0$. This is simply the gauge transformed homogeneous ground state without superflow. On the other hand, the fact that we found an instability at finite momentum in the temperature range $T^{*}<T<\tilde{T}$ could indicate that there is a spatially modulated (metastable) phase even in the range $T^{*}<T<T_{c}$, namely a striped superfluid. Due to the smooth appearance of the unstable mode we expect the transition at $T^{*}$ to that phase to be 2nd order, although this should be studied in detail by constructing the correct inhomogeneous background. The explicit construction of this phase goes however substantially beyond the purpose of this work and we leave this question open for further investigation.

\section{Longitudinal conductivities in the $U(1)$ sector}

In this section we compute the conductivities in the $(0)-(3)$ sector in the presence of superfluid velocity. As far as we are aware, only the transverse conductivities have been computed so far (see for instance [161, 162]). In contrast, here we will focus on the longitudinal conductivities. These are calculated, via the Kubo formula

$$
\sigma=\frac{i}{\omega}\left\langle J^{x} J^{x}\right\rangle
$$

from the two point function

$$
\mathcal{G}_{I J}=\lim _{\Lambda \rightarrow \infty}\left(\mathcal{A}_{I M} \mathcal{F}_{k J}^{M}(\Lambda)^{\prime}\right)
$$

where the matrix $\mathcal{A}$ can be read off from the on-shell action. $\mathcal{F}$ is the matrix valued bulk-to-boundary propagator normalized to the unit matrix at the boundary. Since we are only interested in the entry of the matrix corresponding to $\left\langle J^{x} J^{x}\right\rangle$ and the matrix $\mathcal{A}$ is diagonal, we just need one element, i.e. $\mathcal{A}_{x x}=-\frac{f(r)}{2}$. In order to construct the bulk-to-boundary propagator one needs a complete set of linearly independent solutions for the perturbations of the scalar and gauge fields. This implies solving the system of equations given in Appendix 11.1 at zero momentum. The method follows closely the one detailed in [51] and the one used extensively in previous chapters. Notice that there is a surviving coupling between the gauge fields and the scalar perturbations mediated by $A_{x}$. This makes the computation of the conductivities more involved than in the case without superflow.

Our results show little deviation from what was found at zero superflow. The most interesting new feature is a low frequency peak which appears due to the coupling between the gauge and the scalar sectors induced by the superfluid velocity. In Figures 9.7 and 9.8 we present the results for different values of $S_{x} / \mu$. As expected the behavior for small superfluid velocity far from the critical temperature is the same as the one obtained in [144]. Close to $T^{*}$ a bump is generated in the real part of the conductivity at $\omega \approx 0$. This indicates the existence of a mode with very small imaginary gap. The mode responsible for this behavior is the pseudo-diffusive mode described previously. Due to the conserved $U(1)$ symmetry of the unbroken phase, there exists a diffusive (gapless) mode in the QNM spectrum of the theory. Once the symmetry is spontaneously broken, this mode develops 

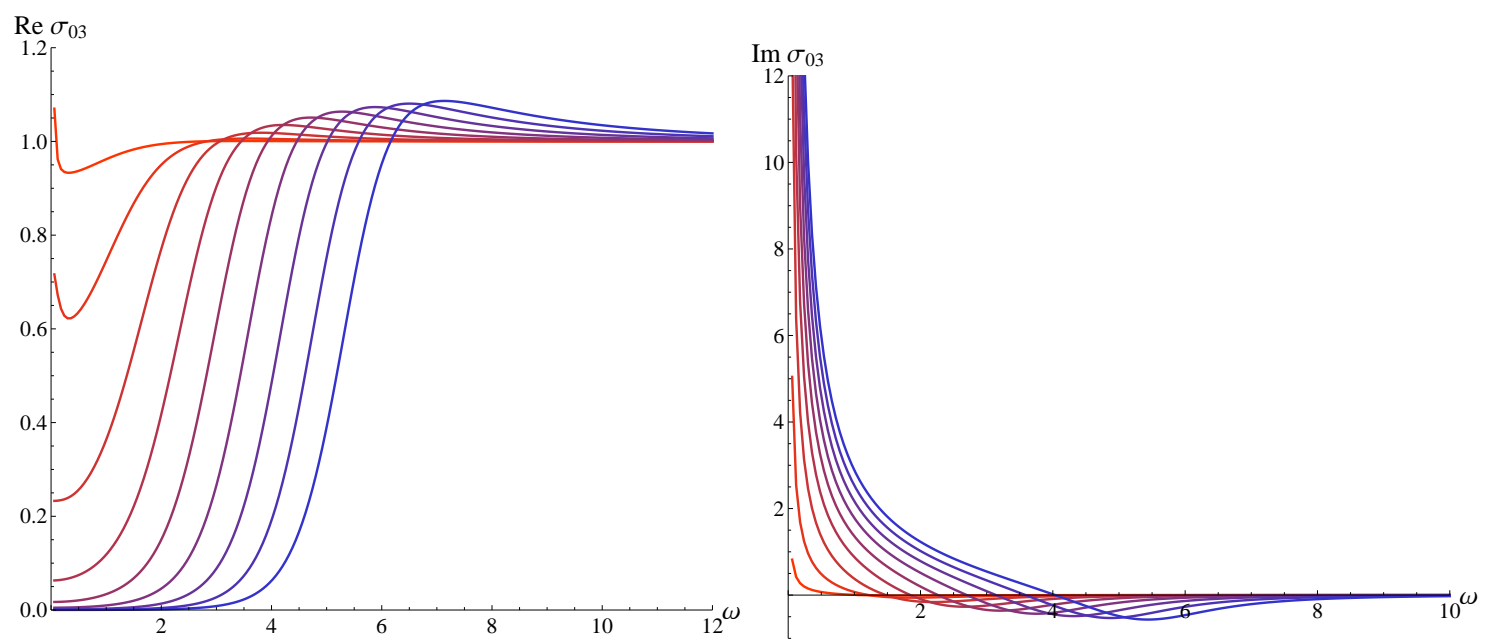

Figure 9.7: Plots of the Real (left) and Imaginary (right) parts of the conductivity for fixed $S_{x} / \mu=0.05$. Different lines correspond to different temperatures from $T=0.99 T_{c}$ (red) to $T=0.38 T_{c}$ (blue).

a purely imaginary gap that increases as we lower the temperature. Therefore, for high enough temperatures below the phase transition, the gap of the pseudo-diffusive mode at $k=0$ is very small and this implies the appearance of a peak at small frequencies in the conductivity as we can see in the figures. If we lower the temperature, the bump starts disappearing simply because the gap of the pseudo-diffusive mode becomes larger. Although this mode was already present in the analysis of the conductivities without superflow, it is only in our present case that it affects the conductivity, due to the coupling at zero momentum between the gauge and scalar sectors mediated by the field $A_{x}$. The size of the peak is proportional to the size of that coupling, i.e. it grows with $S_{x} / \mu$.

\subsubsection{Landau criterion for holographic Type II Goldstone bosons}

In the previous section we studied the lowest lying QNM contained in the (0) - (3) or $U(1)$ sector of the theory for various values of the superfluid velocity and arbitrary angle between the momentum and the direction of the superflow. In this section we extend the analysis to the (1) - (2) sector, which is particular of the $U(2)$ model and contains a type II Goldstone boson in the spectrum, whose dispersion relation is given by 8.2 .92$)$ in the hydrodynamic limit.

The equations describing the system can be found in Appendix B. In this case we choose the momentum to lie always in the direction opposite to the superflow, because as we will see this mode is always unstable. Along with the scalar perturbations prescribed by (9.0.17) we have to consider the following gauge perturbations in the (1) - (2) sector

$$
\begin{aligned}
& A^{(1)}=a_{t}^{(1)}(t, r, x) d t+a_{x}^{(1)}(t, r, x) d x, \\
& A^{(2)}=a_{t}^{(2)}(t, r, x) d t+a_{x}^{(2)}(t, r, x) d x .
\end{aligned}
$$

Again we use the determinant method of [51] to find the QNMs in this sector. Our results are summarized in Figure 9.9, where the dispersion relation for the lowest QNM mode is shown at a particular superfluid velocity. We checked that the result is qualitatively the same for arbitrary $S_{x} / \mu$.

The type II Goldstone mode becomes unstable for arbitrarily small superfluid velocities and temperatures below $\tilde{T}$. However, an important difference arises with respect to the 

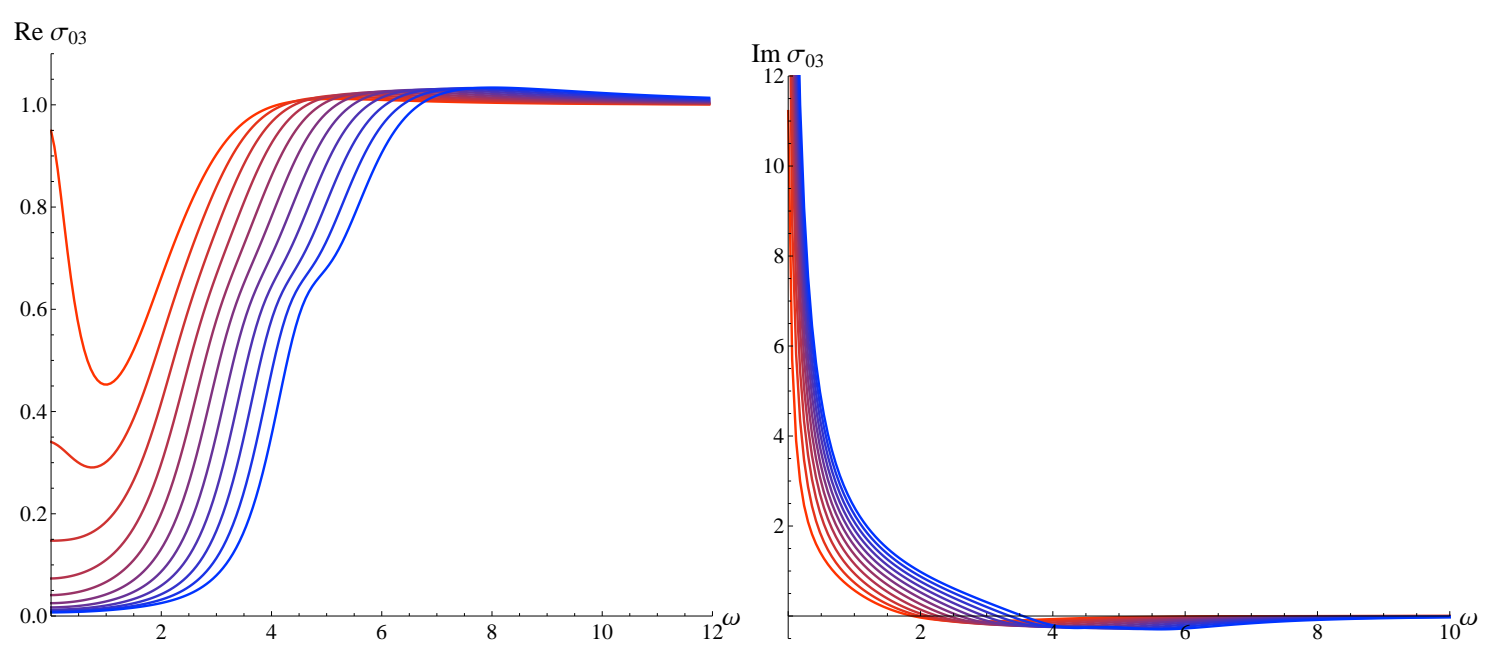

Figure 9.8: Real (left) and imaginary (right) parts of the conductivity for fixed $S_{x} / \mu=0.4$. Different lines correspond to different temperatures in the range $T=0.35 T_{c}$ (blue) $-0.65 T_{c}$ (red).

$U(1)$ sector. The tachyonic mode does not become stable at any temperature below $\tilde{T}$, contrary to the situation in the $(0)-(3)$ sector, there is no analogous of $T^{*}$ in this sector. This behavior can be easily interpreted as a reflection of the Landau criterion of superfluidity in our holographic setup: according to (9.0.1), the critical velocity is zero in any system featuring type II Goldstone bosons, hence for any $T<\tilde{T}$ the superfluid phase is not stable at any finite superfluid velocity. In addition notice that the maximum in the imaginary part occurs at higher values of the momentum as we lower the temperature. In fact as we can see from the figure, lowering the temperature below $\tilde{T}$ the maximum in $\operatorname{Im}(\omega)$ first increases but then starts to decrease again as the temperature is lowered. At the same time it moves out to ever larger values of the momentum.
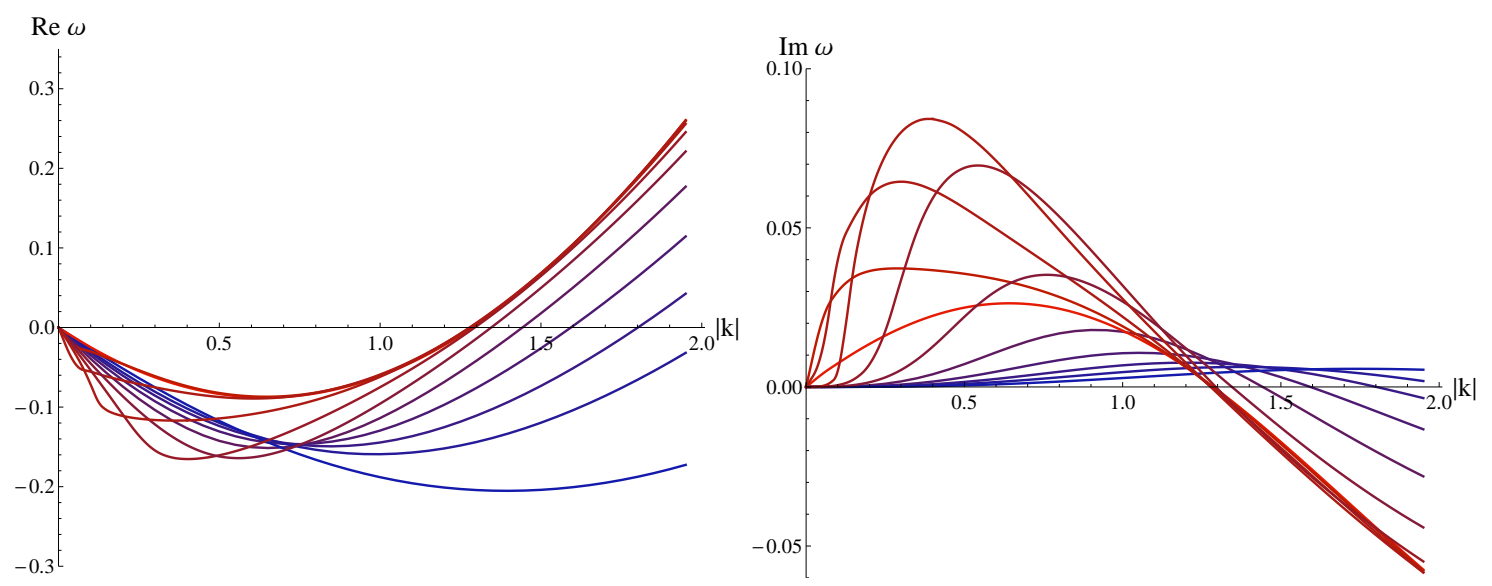

Figure 9.9: Real (left) and imaginary (right) parts of the dispersion relation of the lowest QNM of the $(1)-(2)$ sector in the gauged model for fixed $S_{x} / \mu=0.15$ and a range of temperatures from $T=\tilde{T}=0.95 T_{c}$ (red) to $T=0.45 T_{c}$ (blue) and momentum anti-parallel to the superfluid velocity.

Note that plots analogous to Figures 9.3 and 9.4 do not make any sense in the $U(2)$ model, since the (1) - (2) sector is unstable at any temperature. 

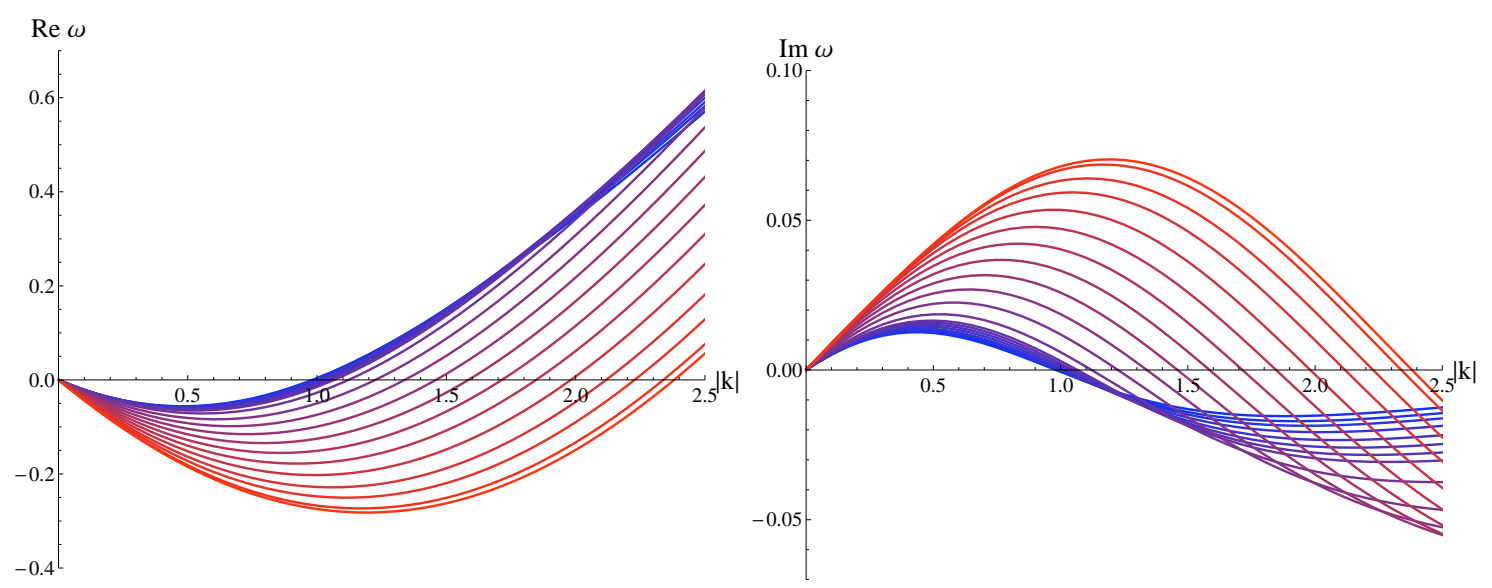

Figure 9.10: Real (left) and imaginary (right) parts of the dispersion relation of the lowest QNM in the (1) - (2) sector of the ungauged model for fixed $S_{x} / \mu=0.25$ and a range of temperatures from $T=\tilde{T}=0.853 T_{c}$ (red) to $T=0.306 T_{c}$ (blue). Momentum is taken anti-parallel to the superfluid velocity.

\section{Ungauged model}

Recall that the ungauged model of Section 8.1 is basically given by the same action (9.0.2) once we keep only the overall $U(1)$ gauge field. Actually it corresponds to the simple $U(1)$ model with two scalar fields with degenerate mass and therefore has an accidental $S U(2)$ global symmetry.

The background solution is again that of the $U(1)$ superfluid, hence the superflow solution can be accommodated also in the ungauged model. The difference is that the type II Goldstone mode appears now in the fluctuations of the upper component of the scalar field $\eta$, whose equation of motion reads

$$
f \eta^{\prime \prime}+\left(f^{\prime}+\frac{2 f}{r}\right) \eta^{\prime}+\left(\frac{\left(\omega+A_{0}\right)^{2}}{f}-\frac{\left(k-A_{x}\right)^{2}}{r^{2}}-m^{2}\right) \eta=0,
$$

and is completely decoupled of all other field fluctuations. As noticed in Chapter 8 the change of the background due to the condensate is enough to trigger the appearance of the type II Goldstone.

It is remarkable that in the ungauged model the type II Goldstone mode is still unstable at any temperature below $\tilde{T}$ for any value of the superfluid velocity. Therefore, the Landau criterion of stability is still valid.

The ungauged model presents a qualitative difference with respect to the gauged model. The value of the momentum at the maximum now decreases as we lower the temperature. This is shown in Figure 9.10, where the dispersion relation of the type II Goldstone at fixed superfluid velocity and for a long range of temperatures is plotted. For arbitrary values of the superfluid velocity we obtained analogous results. 


\section{Chapter 10}

\section{Holographic s+p Superfluids}

An interesting problem in the arena of unconventional superfluids and superconductors is that of the competition and coexistence of different order parameters [170]. A paradigmatic example in the realm of superfluids is that of ${ }^{3} \mathrm{He}$. At low temperature ${ }^{3} \mathrm{He}$ presents two distinct superfluid phases, denoted as $A$ and $B$ phases [171]. ${ }^{3} \mathrm{He}-B$ is the low temperature (and low pressure) phase and it corresponds to a $p$-wave superfluid, where the order parameter transforms as a vector under spatial rotations. ${ }^{3} \mathrm{He}-A$ is the higher temperature (and pressure) superfluid phase. It is a chiral $p$-wave superfluid whose order parameter is a complex vector, and time-reversal and parity symmetry are spontaneously broken. In the domain of unconventional superconductors it has been shown in [172 that for doped three dimensional narrow gap semiconductors such as $\mathrm{Cu}_{x} \mathrm{Bi}_{2} \mathrm{Se}_{3}$ and $\mathrm{Sn}_{1-x} \mathrm{In}_{x}$ Te there is a competition between $s$ and $p$-wave superconducting states. Dialing the coupling constants of the two different channels (corresponding to the $s$ and $p$ pairings) leads to a phase diagram where both a $p$ and an $s$-wave phase exist. Moreover, at the interface of both phases a new $p+i s$ state appears. The order parameter for this phase is the combination of a vector and a pseudoscalar, and breaks both time-reversal and parity symmetry, making this state an interesting example of a topological superconductor 1 .

In AdS/CFT, coexistence and competition of several order parameters has also been addressed holographically in $163,165,174,176,176,177,177,178,178$. In Section 8.2.5 we found that the superfluid phase is unstable at low temperatures and argued that this instability signaled the appearance of a non-trivial $p$-wave order parameter. In this chapter we confirm that prediction and explicitly construct the solutions in which condensation of a vector mode breaks the remaining $U(1)$ and gives rise to a new phase with two condensates: the $s+p$-wave holographic superconductor. The study of these new solutions allows us to determine the phase diagram of the two-component superfluid.

If one works in the grand canonical ensemble, where the chemical potential of the boundary theory is held fixed, the temperature of the system is given by $T \propto 1 / \mu$, where $\mu$ is a dimensionless chemical potential related to that of the boundary theory by rescalings. The final picture is the following: at small enough chemical potential $\mu$ (high temperature) the system is in the normal phase where no condensate is present. For $\mu$ greater than a critical value $\mu_{s}$ the scalar field acquires an expectation value and the system enters the $s$-wave superfluid phase. Going to even larger chemical potential a new phase transition

\footnotetext{
${ }^{1}$ This is actually an example of an axionic state of matter. This $p+i s$ phase belongs to the class D in the classification 173 of 3D topological superconductors. It possesses gapped Majorana fermions as edge states which give rise to an anomalous surface thermal Hall effect. It would be very interesting to realize holographically this axionic superconducting state (see 174 for a holographic time-reversal symmetry breaking $p+i p$ superconductor).
} 
happens: at $\mu_{s p}>\mu_{s}$ a vector condensate appears and for $\mu>\mu_{s p}$ the system is in an $s+p$-wave phase with both scalar and vector non-vanishing order parameters.

Finally, we shall study new configurations of the system where the two chemical potentials corresponding to the two $U(1) \mathrm{s} \subset U(2)$ are switched on. This setup, where the $U(2)$ is explicitly broken to $U(1) \times U(1)$, realizes an unbalanced mixture, characterized by the presence of two species of charges with different chemical potentials. Examples of such systems are unbalanced Fermi mixtures [179], and QCD at finite baryon and isospin chemical potential [180,181]. Moreover, unbalanced superconductors are interesting systems where anisotropic and inhomogeneous phases are expected to appear 182 185]. Holographic realizations of unbalanced systems where only one kind of order parameter can be realized have been constructed in 155,186 . Here we construct new solutions of the system (8.2) corresponding to unbalanced mixtures that allow for competition of different order parameters. We determine its phase diagram as a function of the two chemical potentials and find that $s$-wave, $p$-wave and $s+p$-wave phases exist.

\subsection{The holographic two-component superfluid}

Let us consider the holographic model of a multi-component superfluid of Chapter 8 , that we reproduce below for the shake of clearness

$$
S=\int d^{4} x \sqrt{-g}\left(-\frac{1}{4} F_{c}^{\mu \nu} F_{\mu \nu}^{c}-m^{2} \Psi^{\dagger} \Psi-\left(D^{\mu} \Psi\right)^{\dagger} D_{\mu} \Psi\right)
$$

with

$$
\begin{aligned}
& \Psi=\sqrt{2}\left(\begin{array}{l}
\lambda \\
\psi
\end{array}\right), \quad D_{\mu}=\partial_{\mu}-i A_{\mu}, \quad A_{\mu}=A_{\mu}^{c} T_{c}, \\
& T_{0}=\frac{1}{2} \mathbb{I}, \quad T_{i}=\frac{1}{2} \sigma_{i} .
\end{aligned}
$$

The system lives in the Schwarzschild-AdS background 8.1.3). We consider the following (consistent) ansatz for the fields in our setup

$$
A_{0}^{(0)}=\Phi(r), \quad A_{0}^{(3)}=\Theta(r), \quad A_{1}^{(1)}=w(r), \quad \psi=\psi(r),
$$

with all functions being real-valued. All other fields in (10.1.1) are set to zero, in particular we set $\lambda=0$ without loss of generality. The resulting equations of motion read

$$
\begin{aligned}
& \psi^{\prime \prime}+\left(\frac{f^{\prime}}{f}+\frac{2}{r}\right) \psi^{\prime}+\left(\frac{(\Phi-\Theta)^{2}}{4 f^{2}}-\frac{m^{2}}{f}-\frac{w^{2}}{4 r^{2} f}\right) \psi=0, \\
& \Phi^{\prime \prime}+\frac{2}{r} \Phi^{\prime}-\frac{\psi^{2}}{f}(\Phi-\Theta)=0, \\
& \Theta^{\prime \prime}+\frac{2}{r} \Theta^{\prime}+\frac{\psi^{2}}{f}(\Phi-\Theta)-\frac{w^{2}}{r^{2} f} \Theta=0, \\
& w^{\prime \prime}+\frac{f^{\prime}}{f} w^{\prime}+\frac{\Theta^{2}}{f^{2}} w-\frac{\psi^{2}}{f} w=0 .
\end{aligned}
$$

In what follows we choose the scalar to have $m^{2}=-2$ and the corresponding dual operator to have mass dimension 2 . 
The UV asymptotic behavior of the fields, corresponding to the solution of equations $10.1 .5-10.1 .8$ in the limit $r \rightarrow \infty$, is given by

$$
\begin{aligned}
& \Phi=\mu-\rho / r+O\left(r^{-2}\right), \\
& \Theta=\mu_{3}-\rho_{3} / r+O\left(r^{-2}\right), \\
& w=w^{(0)}+w^{(1)} / r+O\left(r^{-2}\right), \\
& \psi=\psi^{(1)} / r+\psi^{(2)} / r^{2}+O\left(r^{-3}\right),
\end{aligned}
$$

where, on the dual side, $\mu$ and $\rho$ are respectively the chemical potential and charge density corresponding to the overall $U(1) \subset U(2)$ generated by $T_{0}$, whereas $\mu_{3}$ and $\rho_{3}$ are the chemical potential and charge density corresponding to the $U(1) \subset S U(2)$ generated by $T_{3} . \psi^{(1)}$ is the source of a scalar operator of dimension 2 , while $\psi^{(2)}$ is its expectation value. Finally $w^{(0)}$ and $w^{(1)}$ are the source and vev of the current operator $J_{x}^{(1)}$ (recall that $A_{\mu}^{(1)}$ is dual to the current $\left.J_{\mu}^{(1)}\right)$. Notice that in a background where $w(r)$ condenses the $S U(2) \subset U(2)$ is spontaneously broken, and moreover spatial rotational symmetry is spontaneously broken too.

\subsection{The $\mathrm{s}+\mathrm{p}$-wave holographic superconductor}

We are looking for solutions of the equations 10.1.5 - 10.1.8 where $\psi, w$, or both acquire non-trivial profiles. We want them to realize spontaneous symmetry breaking so we impose that their leading UV contributions (dual to the sources of the corresponding operators) vanish. We will switch on a chemical potential $\mu$ along the overall $U(1)$, while requiring that the other chemical potential $\mu_{3}$ remains null. Therefore our UV boundary conditions are

$$
\psi^{(1)}=0, \quad w^{(0)}=0, \quad \mu_{3}=0 .
$$

In the IR regularity requires $A_{t}$ to vanish at the $\mathrm{BH}$ horizon.

We have looked for numerical solutions with non-zero $\psi$ and $w$, shooting from the IR towards the UV where we impose the boundary conditions (10.2.13). We have found the following solutions:

Normal phase: for all values of $\mu$ there exists an analytic solution where $\psi=w=\Theta=0$ and $\Phi=\mu(1-1 / r)$. This solution describes the normal state of the system.

$s$-wave phase: for $\mu \geq \mu_{s} \approx 8.127$ we find solutions with non-zero $\psi$. As seen in previous chapters for these solutions the equations decouple into two sectors: one corresponding to the Abelian holographic superconductor 144 and the other to the unbroken $U(1)$ symmetry. Although $\mu_{3}$ is zero as required in (10.2.13), both charge densities $\rho$ and $\rho_{3}$ are non-vanishing and therefore a two-component s-wave superfluid is realized. Indeed as one can see in eq. 10.1.7) a non-trivial scalar $\psi$ acts a a source for the field $\Theta(r)$, and therefore the only pure s-wave solutions satisfying the boundary conditions 10.2 .13 are those with $\rho_{3} \neq 0$. Hence two different charge densities $\left(\rho\right.$ and $\left.\rho_{3}\right)$ corresponding to the two different $U(1) \mathrm{s} \subset U(2)$ are turned on for these solutions and it is in this sense that this phase was denoted a two-component holographic superfluid ${ }^{2}$.

$s+p$-wave phase: for $\mu \geq \mu_{s p} \approx 20.56$ there are solutions satisfying 10.2 .13 with nonzero $\psi$ and $w$. In these solutions the $U(2)$ symmetry is completely broken, and moreover

\footnotetext{
${ }^{2}$ From eqs. 10.1.5-10.1.7), one can see that the scalar condensate is only charged under a linear combination of $\Phi$ and $\Theta$, whereas in the absence of a vector condensate, the orthogonal combination completely decouples corresponding to the unbroken $U(1)$ gauge field.
} 
since $w^{(1)} \sim\left\langle J_{x}^{(1)}\right\rangle$ spatial rotational symmetry is broken too. Again $\mu_{3}=0$ while $\rho$ and $\rho_{3}$ are non-vanishing, thus realizing an $s+p$-wave phase of a two-component superfluid. Usually $p$-wave superconductivity is triggered by a $\mu_{3}$ chemical potential 145, 187. Here instead the $p$ component of the $s+p$ superfluid is supported by the spontaneously induced charge density $\rho_{3}$. For that reason no solutions with only $p$ condensate are present in this system 3

In figure 10.1 we plot the condensates $\left\langle O_{2}\right\rangle \sim \psi^{(2)}$ and $\left\langle J_{x}^{(1)}\right\rangle \sim w^{(1)}$ as a function of the chemical potential. Notice that the solution where both condensates coexist extends down to as low $1 / \mu$ (or equivalently low temperatures) as where we can trust the decoupling limit and thus neglect backreaction.

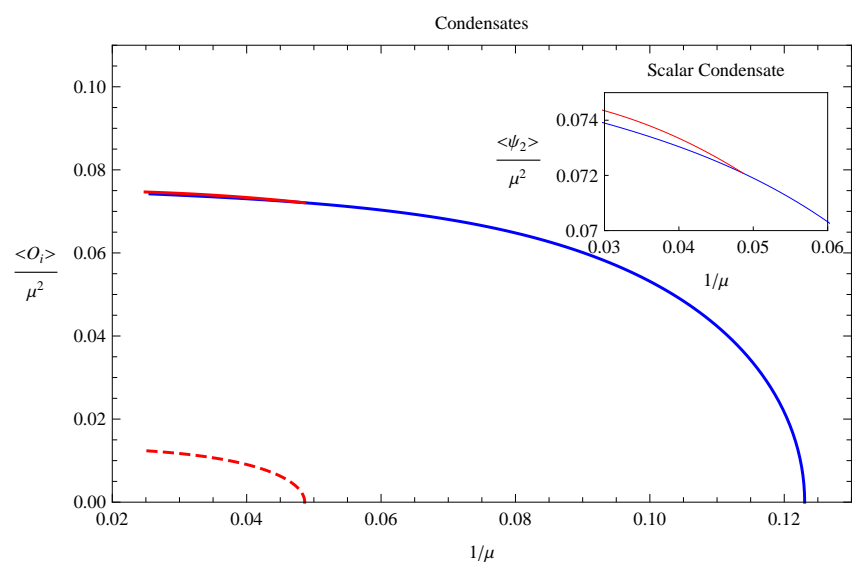

Figure 10.1: Condensates $\psi^{(2)}$ (solid) and $w^{(1)}$ (dashed) as a function of $1 / \mu$ in the $s$-wave (blue) and $s+p$-wave (red) phases. The $p$ condensate appears at $\mu_{s p}$ such that $\mu_{s} / \mu_{s p}=0.395$ as found in Section 8.2.5. The inset zooms in on the plot of $\psi^{(2)}$ to show the difference in the scalar condensate between the $s$ (blue) and the $s+p$ (red) solutions.

To determine the phase diagram of our system we compute the free energy of the different solutions and establish which is preferred when more than one exist. The free energy density is given by the on-shell action, and for our ansatz it reads

$$
\begin{aligned}
F & =-\frac{T}{V} S_{E}=-\frac{1}{2}\left(\mu \rho+\mu_{3} \rho_{3}\right)+ \\
& +\int \frac{d r}{2 f}\left(-f w^{2} \psi^{2}+r^{2}(\Phi-\Theta)^{2} \psi^{2}+\frac{f}{r^{2}} w^{2} \Theta^{2}\right) .
\end{aligned}
$$

The free energy for the different solutions is shown in figure 10.2. At small chemical potential only the normal phase solution exists. At $\mu=\mu_{s} \approx 8.127$ there is a second order phase transition to the $s$-wave solution. If one keeps increasing $\mu$, at $\mu_{s p} \approx 20.56$ there is a

\footnotetext{
${ }^{3}$ It is clear from eq. $\sqrt{10.1 .8}$ that the p-wave condensate only couples directly to the $U(1) \subset S U(2)$, i.e to $\Theta(r)$. Actually, this equation reduces to that of the standard p-wave holographic superconductor [145 187] when the scalar is switched off. As in 145 187, only a non-zero $\Theta$ in the bulk can source the vector condensate since the coupling to the scalar $\psi$ increases the effective mass of $w$ and therefore hinders condensation. In contrast to the standard p-wave scenario we are fixing $\mu_{3}=0$, but solutions with nonzero $\Theta$ are still possible in presence of the s-wave condensate (realized by a non-zero $\psi$ ) as explained above.
} 
second order phase transition from the $s$-wave phase to the $s+p$-wave phase. The system stays in the $s+p$-wave phase for $\mu>\mu_{s p}$.

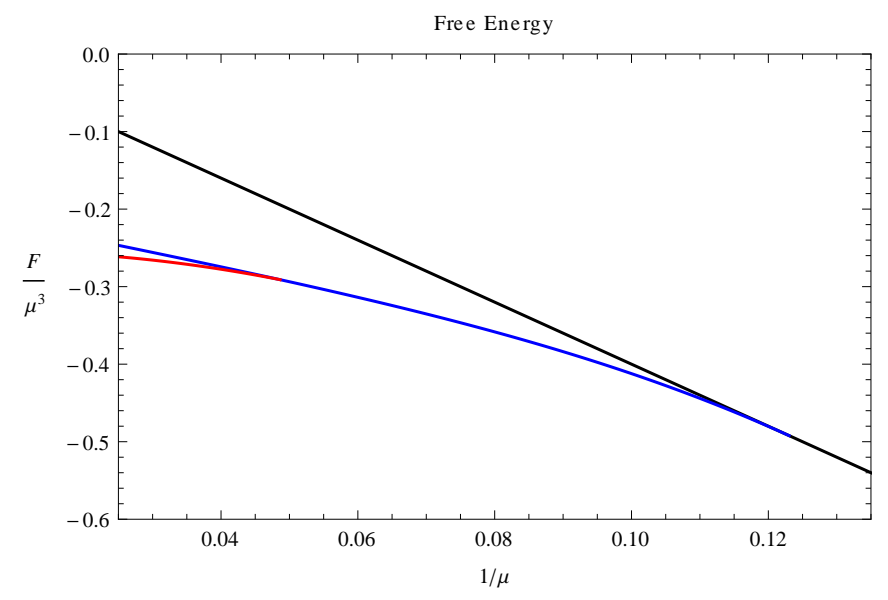

Figure 10.2: Free energy of the different solutions versus $1 / \mu$ : normal phase in black, $s$-wave phase in blue, and $s+p$-wave phase in red.

\subsection{Unbalanced Superconductors}

In this section we relax the condition $\mu_{3}=0$ and study the phase diagram of the system as a function of $\mu$ and $\mu_{3} / \mu$. Notice that turning on a second chemical potential means to explicitly break $U(2) \rightarrow U(1) \times U(1)$. The system can now be interpreted as a holographic dual to an unbalanced mixture [155, 186].

Now that the $U(2)$ is explicitly broken, we can not generically impose that $\lambda=0$ by using gauge transformations. Therefore, in principle both components of the scalar doublet may condense. In [188] it was studied which option is thermodynamically favored. Following their analysis, choosing the condensate to be on the lower component forces us to set $\mu_{3} / \mu<0$ for the solutions to be stable.

The UV boundary conditions now read

$$
\psi^{(1)}=0, \quad w^{(0)}=0 .
$$

As before we use numerical integration to solve the system 10.1.5 - 10.1.8. We are presented with a scenario where four different solutions exist:

Normal phase: an analytic solution where $\psi=w=0, \Phi=\mu(1-1 / r)$ and $\Theta=$ $\mu_{3}(1-1 / r)$ exists for any value of $\mu$ and $\mu_{3}$, and it describes the normal state of the system.

$s$-wave phase: for $\mu-\mu_{3} \geq 8.127$ we find solutions with non-zero $\psi$ resembling those in the balanced case.

$p$-wave phase: for $\left|\mu_{3}\right| / \mu \geq 3.65 / \mu$ solutions with $\psi=0$, but $w \neq 0$ satisfying (10.3.15) exist. The scalar condensate $\left\langle O_{2}\right\rangle$ is null while $\left\langle J_{x}^{(1)}\right\rangle \neq 0$. These solutions break the $U(1) \times U(1)$ down to $U(1)$ and also break the $S O(2)$ corresponding to spatial rotations. Notice that $w(r)$ is not charged under the overall $U(1)$ and therefore this solution is insensitive to the value of $\mu$. This would change if the backreaction of the matter fields on the geometry was taken into account as in [155, 186].

$s+p$-wave phase: for small values of $\mu_{3} / \mu$ we find the extension of the $s+p$-wave solution 
found in the previous section for $\mu_{3}=0$. However, the larger $\left|\mu_{3}\right| / \mu$ the larger the $\mu$ at which the phase appears. We have also found solutions with two condensates in an intermediate region in which $\mu_{3}$ is large and $\mu$ is close to the critical value $\mu_{s}$. But they are always energetically unfavored with respect to the pure $s$-wave solutions (see Figure 10.3 .

By computing the free energy 10.2.14 of the different solutions we determine the phase diagram of the system as a function of $1 / \mu$ and $\mu_{3} / \mu$ which we plotted in figure 10.3 For small values of $\mu_{3} / \mu$ the situation is very similar to what we found in the previous section for $\mu_{3}=0$. As already mentioned, as $\left|\mu_{3}\right| / \mu$ gets larger, the transition to the $s+p$-wave phase happens at a higher value of $\mu$. It might be the case that the phase eventually disappears at a finite value of that ratio, but this would happen beyond the region of applicability of the decoupling limit, and thus backreaction should be taken into account ${ }^{4}$. For $\left|\mu_{3}\right| / \mu$ large enough, the $p$-wave phase is preferred at intermediate values of $\mu$. Therefore, as $\mu$ is increased above a critical value $\mu_{p}$ the system goes from the normal to the $p$-wave phase through a second order phase transition. If $\mu$ is increased even further a first order phase transition takes the system from the $p$-wave to the $s$-wave phase. This $p$ - to $s$-wave first order phase transition is illustrated by figure 10.4 where we plot the free energy of both phases (and that of the normal phase) as a function of $\mu$ at a fixed value of $\mu_{3} / \mu=-1$. The tricritical point where the normal, $s$-wave and $p$-wave phases meet happens at $1 / \mu \approx 0.223$ and $\left|\mu_{3}\right| / \mu \approx 0.815$. The $p$-wave solution is never energetically preferred for $\left|\mu_{3}\right| / \mu<0.815$.

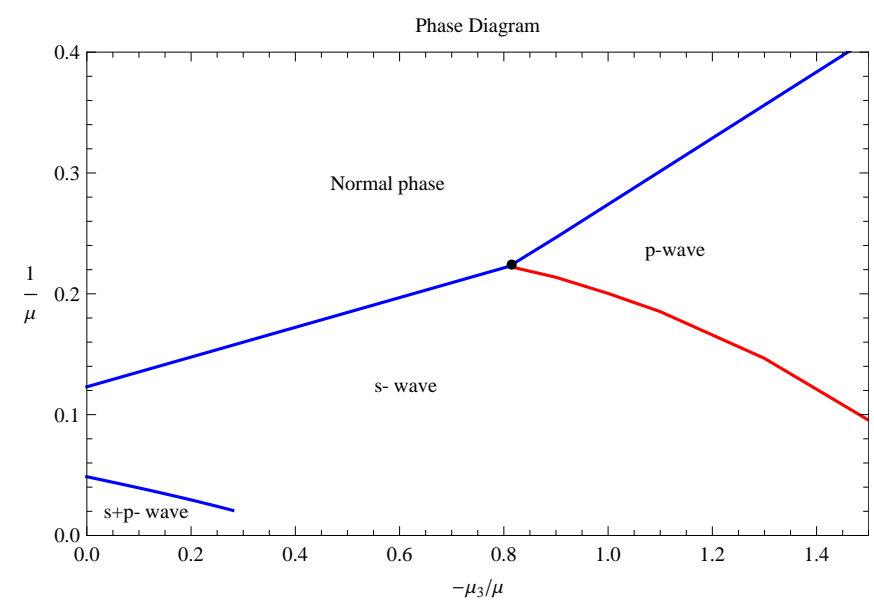

Figure 10.3: Phase diagram of the unbalanced system as a function of $1 / \mu$ and $\mu_{3} / \mu$. Second order phase transitions are denoted by blue lines, whereas the red line corresponds to a first order phase transition.

A cautionary comment about the phase diagram of figure 10.3 is in order. In the regions of the parameter space where $\left|\mu_{3}\right| / \mu \gg 1$ or $1 / \mu \ll 1$ the probe limit is not valid anymore, and therefore the phase diagram might be modified once backreaction is taken into account 5 . Indeed, the nature of the different phase transitions, as well as the critical

\footnotetext{
${ }^{4}$ Notice that if the $s+p$-wave phase survived down to $1 / \mu=0$ for $\mu_{3} / \mu$ lower than a critical value (as the phase diagram 10.3 seems to imply) we would be in the pressence of a quantum critical point at which the system goes from the $s+p$ to the $s$-wave phase. This resembles what happens in 172 for the $p+i s$ superconductor.

${ }^{5}$ Remember that the decoupling limit corresponds to taking the gauge coupling (and charge of the scalar
} 
values of the chemical potentials could be altered in those regions [189, 190]. However, in $2+1$-dimensions both the $s$-wave and $p$-wave superconducting phase transitions separately are known to remain second order even lor large backreaction [155, 186]. Therefore, we expect the main features of the phase diagram like the existence of distinct $s$ and $p$ wave phases meeting at a tricritical point will not be very sensitive to backreaction. The order of the phase transition between the $s$ and $p$-wave phases could still be modified by backreaction.

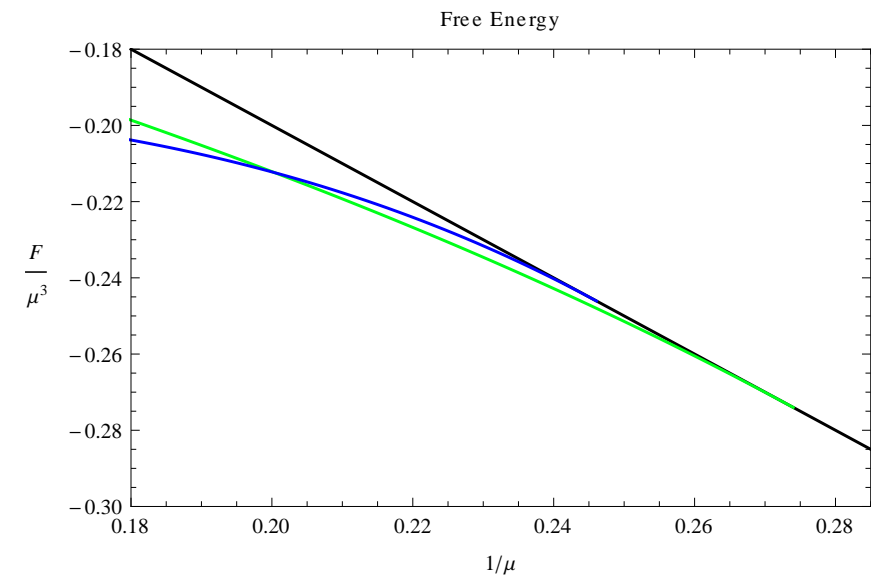

Figure 10.4: Free energy as a function of $1 / \mu$ for $\mu_{3} / \mu=-1$. Black corresponds to the normal phase, blue to the $s$-wave phase, and green to the $p$-wave phase.

field) $g_{\mathrm{YM}}$ to be very large, so the effect of the matter fields on the metric is negligible. Hence it is valid as far as $\mu_{i} \ll g_{\mathrm{YM}}$ and the condensates are small. 


\section{Chapter 11}

\section{Discussion and Outlook}

In this second part we have presented several results on exotic superfluids, by means of holographic methods.

In Chapter III the main focus was to establish the existence of type II Goldstone modes in the quasinormal mode spectrum of a holographic theory dual to a strongly coupled superfluid with $U(2)$ symmetry. We studied two models, one in which only the overall $U(1)$ symmetry is gauged in the AdS bulk (ungauged model) and another in which all the $U(2)$ symmetry is gauged. The most important finding is that indeed there exist ungapped excitations represented by quasinormal modes in the AdS bulk that show the expected but somewhat unusual quadratic dispersion relation of type II Goldstone bosons.

Section 8.1 is devoted to the analysis of the ungauged model. Since the well-known s-wave superconductor is a subsector of both the ungauged and the gauged model we also briefly review first the findings of [2]. One of the main results of [2] concerning the $U(1)$ superfluid was that the QNM spectrum in the superfluid phase contains such an ungapped Goldstone mode with dispersion relation $\omega= \pm v_{s} k+O\left(k^{2}\right)$. This mode can also be understood as the sound mode of the superfluid and $v_{s}$ is the sound velocity In the non-backreacted model these are the only hydrodynamic modes in the broken phase. Recall that in the unbroken phase in contrast there exists a single hydrodynamic mode signaling the usual diffusive behavior of a normal fluid. Its dispersion relation is $\omega=-i D k^{2}$, where $D$ is the diffusion constant. This mode develops a purely imaginary gap $\omega=-i \gamma-i \tilde{D} k^{2}$ in the unbroken phase. This is quite natural because the single purely imaginary mode can not move off the imaginary axis? ${ }^{2}$. The hydrodynamics of the broken phase is fully captured by the Goldstone mode and the diffusion mode does the simplest thing it can to drop out of the hydrodynamic regime by growing the gap $\gamma$. Since this purely imaginary gapped mode has its origin in the universal diffusive mode of the unbroken phase we expect that it is a universal feature of a large class of superfluids, not only holographic ones. This mode will necessarily dominate the late time response in the order parameter to homogeneous perturbations and in regimes close but below the critical temperature where the gap $\gamma$ is rather small. Therefore the order parameter is bound to show a purely exponential decay towards its equilibrium value without any oscillation. In contrast for lower temperatures where $\gamma$ becomes large there are other low lying QNMs

\footnotetext{
${ }^{1}$ In 102 it was pointed out that this mode corresponds to the fourth sound (see also Section 4.2 ).

${ }^{2}$ Quasinormal modes are bound to come either in pairs $\omega_{n}$ and $\tilde{\omega}_{n}=-\omega_{n}^{*}$ or are fixed on the imaginary axis. This follows from rather generic symmetry considerations for retarded Green's functions, see appendix 11.3
} 
with real and imaginary parts in their frequency. In this low temperature regime the response in the order parameter is then an exponentially damped oscillation rather than a purely imaginary decay. This universal aspect of the late time response of superfluids was also emphasized in recent numerical studies of quenches of holographic superfluids in 152 .

Then we show that even with this drastic simplification, i.e. not gauging the global $S U(2)$ symmetry in the bulk, the model presents Goldstone modes with quadratic dispersion relation. Hence, within this model a type II NG boson is found as a consequence of having broken just one charge generator (the one associated to the $U(1)$ symmetry).

For the ungauged model this does constitute a surprising result. After all, the field theory dual to this model does not contain the necessary conserved currents that would correspond to the generators of the global $S U(2)$ symmetry. Standard proofs of the Goldstone theorem take the existence of such conserved currents for granted. On the other hand it is basically guaranteed that one can construct an effective field theory, a simple Landau-Ginzburg type model, that captures the essential dynamics of the light modes, i.e. the lowest lying quasinormal modes. Such a model would be essentially given by the field theoretical model of Section 4.4.1 and this guarantees the existence of the type II Goldstone modes. However one can expect that such an effective field theory approach can capture only the physics of the low lying QNMs but not the higher modes. This is indeed what happens: the partner mode of the type II Goldstone mode in the ungauged model does not behave in the supposed universal way $\omega=q \mu$. In contrast the corresponding mode in the gauged model does obey this relation approximately and the deviation we found could very well be attributed to numerical difficulties and uncertainties that arise in the study of the higher QNMs.

One rather interesting perspective on the ungauged model opens up if we vary the masses of the scalar fields in the AdS bulk. If the masses are slightly different, then at the critical temperature only one of the two scalars will feature an ungapped QNM (the one with smaller mass). The lowest scalar mode of the second one will still be gapped at that temperature. As one goes through the phase transition we do not expect this mode to become massless at lower temperatures. Rather it should become a pseudo-Goldstone mode with a gap that is proportional to the mass splitting. The appearance of the type II Goldstone mode can then be interpreted as the effect of a symmetry enhancement at the point in parameter space where the masses of the scalars become degenerate. Since this symmetry is not represented by bulk-gauge fields we might call it an accidental symmetry. At this point it is difficult to resist the temptation to draw a parallel to the conjectured symmetry enhancement of high $T_{c}$ superconductors. In 170 it was suggested that the phase diagram of high $T_{c}$ superconductors can be captured by a unified model with and enhancement of the $S O(3) \times U(1)$ symmetry of rotations and electromagnetism to a larger $S O(5)$ symmetry. Since high $T_{c}$ superconductors are d-wave rather than s-wave it remains to be seen how our symmetry enhancement mechanism and the resulting type II Goldstone mode can be combined with holographic models of d-wave superfluids such as $146,147{ }^{3}$.

In Section 8.2 we studied the fully gauged $U(2)$ model. There we analyze the fluctuation equations to linear order, which decompose into three decoupled sectors. One being the already encountered $U(1)$ s-wave superfluid, the other describing the non-Abelian sector in which the type II Goldstone mode resides and a third one with the unbroken $U(1)$

\footnotetext{
${ }^{3}$ The appearance of unexpected massless modes related to symmetry enhancement in the context of Bose condensates was as well found in 191
} 
symmetry. We find several important differences compared to the ungauged model. The most eye-jumping one is that now we can also define and study the full set of conductivities corresponding to the $U(2)$ symmetry. Nothing special occurs of course in the unbroken phase, there are simply four diagonal conductivities for all the four bulk gauge fields. In the broken phase there are however interesting new phenomena. In particular there are now off-diagonal conductivities that do not simply vanish. In addition we have found that also the diagonal conductivities in the $(1)-(2)$ sector, the one containing the type II Goldstone mode, have delta-function poles at zero frequency. In this sense this sector is still superconducting. Moreover, going to a decoupling basis for this sector leads to a very suggestive result: the conductivity develops a Drude-like peak characteristic of metals on top of the infinite DC conductivity. On the other hand Landau's criterion for superfluidity does not hold in this sector. Recall that this says that superfluidity takes place for flow velocities $v$ that are smaller than the critical velocity $v_{c}$ where $v_{c}=\min _{i} \omega_{i}(k) / k$ for all excitation branches $i$ and over all momenta $k$ [157, 192]. For a type II Goldstone mode the critical flow velocity is clearly zero.

A second difference concerns the fate of the diffusive modes. In the unbroken phase there are simply four diffusive modes, one for each gauge field in the AdS bulk. In the broken phase there is one purely imaginary gapped 'pseudo-diffusive' mode in the (0) - (3) sector, i.e. in the sector isomorphic to the $U(1)$ s-wave superfluid. Since there is still one unbroken $U(1)$ symmetry there is also a normal diffusive mode for the preserved $U(1)$ symmetry. In the (1) - (2) sector we have however two diffusive modes in the unbroken phase. Going through the phase transition these two modes can pair up and move off the imaginary axis, becoming a pair of usual gapped quasinormal modes with real and imaginary parts in their frequencies. Generically the imaginary part of this gap is smaller (i.e. it lies closer to the real axis) then the gap of the purely imaginary mode in the $(0)-(3)$ sector. A large, generic perturbation will in its late time response pattern excite both the $(0)-(3)$ and the $(1)-(2)$ sector. The late time response of the $U(2)$ invariant order parameter $\sqrt{\left|\mathcal{O}_{1}\right|^{2}+\left|\mathcal{O}_{2}\right|^{2}}$ will therefore be dominated by these paired modes and show an oscillatory behavior in contrast to the response pattern of the order parameter in the $U(1)$ case 152 .

Another remarkable QNM is the special gapped mode, i.e. the partner mode of the type II Goldstone boson. At very high temperatures this mode and the one which at $T=T_{c}$ leads to the sound mode are degenerate. As we lower the temperature the gap of these modes becomes different and, for $T<T_{c}$, it is expected that $\operatorname{Re}(\omega(k=0))$ for the Special Gapped mode is proportional to $q \mu$ 115, 118. In particular we find $\omega \sim 1.1 \mu$ even if $q=1$ in our conventions. Unfortunately with the numerical methods employed here we found it very difficult to study this mode and the discrepancy can therefore very well be a consequence of insufficient numerical accuracy. It is probably worth the effort to study this mode with alternative methods such as the relaxation method developed in 193,194 .

In Chapter 9 we have analyzed the holographic realization of the Landau criterion of superfluidity. The study was motivated by the appearance of type II Goldstone bosons in the model (9.0.2). The quadratic nature of the dispersion relation of the type II Goldstone mode should be responsible for driving the system out of the superfluid phase for arbitrarily small superfluid velocity.

Taking advantage of the fact that the usual $U(1)$ holographic s-wave superconductor is contained in $(9.0 .2)$, we have revisited the Landau criterion for holographic type I Goldstone modes. When addressing the question of the stability of the condensate at finite superfluid velocity the analysis of the free energy does not give the correct answer. The QNM 
spectrum contains a tachyonic mode at finite momentum for temperatures $T^{*}<T<\tilde{T}$. As defined $\tilde{T}$ is the temperature at which free energies of the normal and condensate phase coincide. In contrast, $T^{*}$ is the temperature where the tachyonic instability arises. Hence, the homogeneous superfluid is stable only for $T<T^{*}$, see Figure 9.6. The results for the sound velocity as a function of the angle $\gamma$ between the propagation direction and the superfluid velocity, depicted in Figures 9.3 and 9.4 , are perfectly consistent with this statement: at $T=T^{*}$ and $\gamma=\pi$ the velocity of sound vanishes. This condition can be seen to be equivalent to the Landau criterion and signals the existence of a critical velocity above which the superfluid is not stable anymore. Since the maximum of the imaginary part of the unstable mode has non-vanishing wave number it is natural to suggest that there might be another, spatially modulated phase for $T>T^{*}$. The nature or this inhomogeneous phase is however unknown.

We have also computed the longitudinal conductivities for various superfluid velocities. As far as we know, they have not been computed before. We see a peak at $\omega=0$, due to the coupling with the spatial component of the gauge field $A_{x}$. The peak decreases as we lower the temperature until it gets completely suppressed (Figure 9.7). We believe that this enhancement of the DC conductivity is caused by the gap of the pseudo-diffusive mode, which in the presence of superfluid velocity is formed due to the coupling between the gauge and scalar sectors that takes place even at $k=0$.

Moving to the (1) - (2) sector, we worked out the impact of the superflow on the type II Goldstone mode. We found that the Landau criterion is effective for arbitrarily small superfluid velocity as depicted in Figure 9.9. The tachyon persists for the whole range of temperatures and (finite) superfluid velocities we have been able to analyze. Hence, we conclude that the critical superfluid velocity for this sector vanishes, in complete accordance with the Landau criterion applied to modes with dispersion relation $\omega \propto k^{2}$. An analogous result holds for the type II Goldstone mode in the ungauged model.

Finally, in Chapter 10 we report on the construction of a holographic $s+p$-wave superconducting state. This phase, where both an $s$-wave and $p$-wave condensates exist, is the preferred state at low temperatures of the holographic two-component superfluid.

The main results are summarized by figures 10.1 and 10.3 . Figure 10.1 shows that an $s+p$-wave state appears at low temperatures. A free energy analysis determined that the system enters this state through a second order phase transition, and stays in it for as low temperature as we can go.

On the other hand, figure 10.3 presents the phase diagram for the unbalanced system: chemical potentials for the two $U(1) \mathrm{s} \subset U(2)$ are turned on, and hence $U(2)$ is explicitly broken to $U(1) \times U(1)$. In this phase diagram three different superconducting phases are present. These are the standard $s$-wave phase where a scalar condensate breaks the $U(1) \times U(1)$ down to $U(1)$; a $p$-wave phase where $\left\langle J_{x}^{(1)}\right\rangle \neq 0, U(1) \times U(1)$ is broken to (a different) $U(1)$, and also spatial rotational symmetry is broken; and an $s+p$-wave phase where the $U(1) \times U(1)$ is completely broken by the $s$ and $p$-wave condensates, and again spatial rotational symmetry is broken. Remarkably, while the system goes from the normal phase to the $s$-wave and $p$-wave phases through second order phase transitions, the phase transition between the $s$ and $p$-wave phases is always a first order one. The existence of this first order phase transition between superconducting phases in the unbalanced system is an interesting prediction of our holographic model. These conclusions could be sensitive to the inclusion of backreaction since, as already mentioned, in principle the order of the phase transitions could change when the parameters are large and the decoupling limit breaks down. Yet in the proximity of the tricritical point, where the $p$ - and $s$-wave 
phases meet, the matter fields and its derivatives are small enough for the probe limit to be trusted. Hence the existence of this point and the first order phase transition between the $p$ - and $s$-wave phases in its proximity will survive once backreaction is considered, at least for large enough gauge coupling. Moreover, a preliminary study of backreacted solutions in that region supports this conclusion and show it holds for small values of the gauge coupling too. In any case, in order to ensure the stability of the different phases it is important to study the quasinormal mode spectrum of the model. As pointed out in Chapter 9, it might be possible that instabilities towards inhomogeneous phases appear.

In 104 a QFT model featuring a gauged $U(2)$ symmetry, and with a symmetry breaking scheme similar to ours is studied. There the autors find roton excitations along the direction of the vector condensate. It would be interesting to study the quasinormal mode spectra of the $s+p$-wave phase and see if something similar happens in our case. We leave this for a future investigation.

For future firections, a possible generalization would be to analyze the model 8.2.34 when the backreaction onto the metric is taken into account. This introduces the energymomentum tensor as an operator of the dual field theory and thus we expect the usual sound and shear modes to stem from bulk metric fluctuations. Moreover, this would allow us to obtain reliable results even at very low temperatures and for instance compute the density of superconducting charge densities at zero temperature, as well as $\mathcal{B}(T=0)$.

We have constructed here a simply model with type II Goldstone bosons using a "bottom-up" strategy. It is however also interesting to ask if such models can be realized via "top-down" D-brane, string theory or M-theory constructions 187, 195 198.

Another possible direction of research involves using the Fluid/Gravity correspondence 38 in order to derive the Hydrodynamic expansion of the current and upon including backreaction also the constitutive relation for the energy-momentum tensor. This will throw light on the hydrodynamic behavior of non-relativistic superfluids and in particular should result in the formulation of the hydrodynamics of relativistic type II Goldstone modes. Up to our knowledge this is not even known to the leading, i.e. zeroth order in derivatives.

Finally it is also interesting to ask the question if holographic models featuring Goldstone modes with higher order dispersion relation $\omega=c k^{n}$ with $n>2$ can be constructed. 


\section{Appendix of Part III}

\subsection{Fluctuation equations in the $(0)-(3)$ sector}

The fluctuations in the $U(1)$ theory or the $(0)-(3)$ sector contain the zeroth and third color sectors of the gauge field and the lower component of the scalar field $\sigma=\rho+i \delta$. The equations of motion for an arbitrary direction of the momentum then read

$$
\begin{aligned}
0= & f \rho^{\prime \prime}+\left(f^{\prime}+\frac{2 f}{r}\right) \rho^{\prime}+\left(\frac{\omega^{2}}{f}+\frac{A_{0}^{2}}{f}-\frac{A_{x}^{2}}{r^{2}}-\frac{|k|^{2}}{r^{2}}-m^{2}\right) \rho+\frac{2 i \omega A_{0}}{f} \delta+2 a_{t}^{(-)} \Psi \frac{A_{0}}{f} \\
& -2 \frac{a_{x}^{(-)}}{r^{2}} \Psi A_{x}+|k| \cos (\gamma) \frac{2 i}{r^{2}} A_{x} \delta \\
0= & f \delta^{\prime \prime}+\left(f^{\prime}+\frac{2 f}{r}\right) \delta^{\prime}+\left(\frac{\omega^{2}}{f}+\frac{A_{0}^{2}}{f}-\frac{A_{x}^{2}}{r^{2}}-\frac{|k|^{2}}{r^{2}}-m^{2}\right) \delta-\frac{2 i \omega A_{0}}{f} \rho-i \Psi \omega \frac{a_{t}^{(-)}}{f} \\
& -|k| \cos (\gamma) \frac{2 i}{r^{2}} A_{x} \rho-|k| \cos (\gamma) \frac{i}{r^{2}} \Psi a_{x}^{(-)}-|k| \sin (\gamma) \frac{i}{r^{2}} \Psi a_{y}^{(-)} \\
0= & f a_{t}^{\prime \prime(-)}+\frac{2 f}{r} a_{t}^{\prime(-)}-\left(\frac{|k|^{2}}{r^{2}}+2 \Psi^{2}\right) a_{t}^{(-)}-\frac{\omega|k|}{r^{2}} \cos (\gamma) a_{x}^{(-)}-\frac{\omega|k|}{r^{2}} \sin (\gamma) a_{y}^{(-)} \\
& -4 \Psi A_{0} \rho-2 i \omega \Psi \delta, \\
0= & f a_{x}^{\prime \prime(-)}+f^{\prime} a_{x}^{\prime(-)}+\left(\frac{\omega^{2}}{f}-2 \Psi^{2}\right) a_{x}^{(-)}+\frac{\omega|k|}{f} \cos (\gamma) a_{t}^{(-)}+2 i|k| \cos (\gamma) \Psi \delta \\
& -4 \Psi \rho A_{x}-\frac{|k|^{2} \sin ^{2}(\gamma)}{r^{2}} a_{x}^{(-)}+\frac{|k|^{2} \cos (\gamma) \sin (\gamma)}{r^{2}} a_{y}^{(-)} \\
0= & f a_{y}^{\prime \prime(-)}+f^{\prime} a_{y}^{\prime(-)}+\left(\frac{\omega^{2}}{f}-2 \Psi^{2}\right) a_{y}^{(-)}+\frac{\omega|k|}{f} \sin (\gamma) a_{t}^{(-)}+2 i|k| \sin (\gamma) \Psi \delta \\
& -\frac{|k|^{2} \cos ^{2}(\gamma)}{r^{2}} a_{y}^{(-)}+\frac{|k|^{2} \cos (\gamma) \sin (\gamma)}{r^{2}} a_{x}^{(-)},
\end{aligned}
$$

and the constraint

$$
0=\frac{i \omega}{f} a_{t}^{(-)}+\frac{i|k|}{r^{2}} \cos (\gamma) a_{x}^{(-)}+\frac{i|k|}{r^{2}} \sin (\gamma) a_{y}^{(-)}+2 \Psi^{\prime} \delta-2 \Psi \delta^{\prime}
$$

where we have used $k_{x}=|k| \cos (\gamma), k_{y}=|k| \sin (\gamma)$. The general pure gauge solution in this sector is

$$
\delta=i \lambda \Psi ; \quad \rho=0 ; \quad a_{t}^{(-)}=\lambda \omega ; \quad a_{x}^{(-)}=-\lambda|k| \cos (\gamma) ; \quad a_{y}^{(-)}=-\lambda|k| \sin (\gamma),
$$

where $\lambda$ is an arbitrary constant.

\subsection{Fluctuation equations in the (1) - (2) sector}

The perturbations in the $(1)-(2)$ sector of the $U(2)$ theory include the fluctuations of the upper component of the scalar field, $\eta=\alpha+i \beta$, along with that sector of the gauge 
field. For momentum in the opposite direction of the superflow, the equations of motion read

$$
\begin{aligned}
& 0=f a_{x}^{\prime \prime(1)}+f^{\prime} a_{x}^{\prime(1)}+\left(\frac{\omega^{2}}{f}-\Psi^{2}+\frac{\left(A_{t}^{(3)}\right)^{2}}{f}\right) a_{x}^{(1)}-2 i \frac{A_{t}^{(3)} \omega}{f} a_{x}^{(2)}+i \omega \frac{A_{x}^{(3)}}{f} a_{t}^{(2)} \\
& -\frac{A_{t}^{(3)} A_{x}^{(3)}}{f} a_{t}^{(1)}-2 A_{x}^{(0)} \Psi \alpha+2 i k \Psi \beta-\frac{i k A_{t}^{(3)}}{f} a_{t}^{(2)}+\frac{\omega k}{f} a_{t}^{(1)}, \\
& 0=f a_{x}^{\prime \prime(2)}+f^{\prime} a_{x}^{\prime(2)}+\left(\frac{\omega^{2}}{f}-\Psi^{2}+\frac{\left(A_{t}^{(3)}\right)^{2}}{f}\right) a_{x}^{(2)}+2 i \frac{A_{t}^{(3)} \omega}{f} a_{x}^{(1)}-i \omega \frac{A_{x}^{(3)}}{f} a_{t}^{(1)} \\
& -\frac{A_{t}^{(3)} A_{x}^{(3)}}{f} a_{t}^{(2)}+2 \Psi A_{x}^{(0)} \beta+2 i k \Psi \alpha+\frac{i k A_{t}^{(3)}}{f} a_{t}^{(1)}+\frac{\omega k}{f} a_{t}^{(2)}, \\
& 0=f a_{t}^{\prime \prime(1)}+\frac{2 f}{r} a_{t}^{\prime(1)}-\left(\frac{\left(A_{x}^{(3)}\right)^{2}}{r^{2}}+\Psi^{2}+\frac{k^{2}}{r^{2}}\right) a_{t}^{(1)}+\frac{A_{t}^{(3)} A_{x}^{(3)}}{r^{2}} a_{x}^{(1)}-i \omega \frac{A_{x}^{(3)}}{r^{2}} a_{x}^{(2)}-2 i \omega \beta \Psi \\
& -2 \phi \Psi \alpha+\frac{i k A_{t}^{(3)}}{r^{2}} a_{x}^{(2)}-\frac{2 i k A_{x}^{(3)}}{r^{2}} a_{t}^{(2)}-\frac{\omega k}{r^{2}} a_{x}^{(1)} \\
& 0=f a_{t}^{\prime \prime(2)}+\frac{2 f}{r} a_{t}^{\prime(2)}-\left(\frac{\left(A_{x}^{(3)}\right)^{2}}{r^{2}}+\Psi^{2}+\frac{k^{2}}{r^{2}}\right) a_{t}^{(2)}+\frac{A_{t}^{(3)} A_{x}^{(3)}}{r^{2}} a_{x}^{(2)}+i \omega \frac{A_{x}^{(3)}}{r^{2}} a_{x}^{(1)} \\
& -\frac{i k A_{t}^{(3)}}{r^{2}} a_{x}^{(1)}+\frac{2 i k A_{x}^{(3)}}{r^{2}} a_{t}^{(1)}-\frac{\omega k}{r^{2}} a_{x}^{(2)}-2 i \omega \alpha \Psi+2 A_{t}^{(0)} \Psi \beta, \\
& 0=f \alpha^{\prime \prime}+\left(f^{\prime}+\frac{2 f}{r}\right) \alpha^{\prime}+\left(\frac{\omega^{2}}{f}+\frac{\left(A_{t}^{(0)}+A_{t}^{(3)}\right)^{2}}{4 f}-\frac{\left(A_{x}^{(0)}+A_{x}^{(3)}\right)^{2}}{4 r^{2}}-\frac{k^{2}}{r^{2}}-m^{2}\right) \alpha \\
& +\left(i \omega\left(\frac{A_{t}^{(0)}+A_{t}^{(3)}}{f}\right)+\frac{i k}{r^{2}}\left(A_{x}^{(0)}+A_{x}^{(3)}\right)\right) \beta+\frac{A_{t}^{(0)} \Psi}{2 f} a_{t}^{(1)}-i \omega \frac{\Psi}{2 f} a_{t}^{(2)} \\
& -\frac{A_{x}^{(0)} \Psi}{2 r^{2}} a_{x}^{(1)}-\frac{i k \Psi}{2 r^{2}} a_{x}^{(2)} \\
& 0=f \beta^{\prime \prime}+\left(f^{\prime}+\frac{2 f}{r}\right) \beta^{\prime}+\left(\frac{\omega^{2}}{f}+\frac{\left(A_{t}^{(0)}+A_{t}^{(3)}\right)^{2}}{4 f}-\frac{\left(A_{x}^{(0)}+A_{x}^{(3)}\right)^{2}}{4 r^{2}}-\frac{k^{2}}{r^{2}}-m^{2}\right) \beta \\
& -\left(i \omega\left(\frac{A_{t}^{(0)}+A_{t}^{(3)}}{f}\right)+\frac{i k}{r^{2}}\left(A_{x}^{(0)}+A_{x}^{(3)}\right)\right) \alpha-\frac{A_{t}^{(0)} \Psi}{2 f} a_{t}^{(2)}-i \omega \frac{\Psi}{2 f} a_{t}^{(1)} \\
& +\frac{A_{x}^{(0)} \Psi}{2 r^{2}} a_{x}^{(2)}-\frac{i k \Psi}{2 r^{2}} a_{x}^{(1)}
\end{aligned}
$$


subject to the constraints

$$
\begin{aligned}
& 0=2 f\left(\Psi \beta^{\prime}-\Psi^{\prime} \beta\right)+a_{t}^{(2)} A_{t}^{\prime(3)}-a_{t}^{\prime(2)} A_{t}^{(3)}+\frac{f}{r^{2}}\left(A_{x}^{(3)} a_{x}^{\prime(2)}-a_{x}^{(2)} A_{x}^{\prime(3)}\right)-i \omega a_{t}^{\prime(1)}-\frac{i k f}{r^{2}} a_{x}^{\prime(1)}, \\
& 0=2 f\left(\Psi \alpha^{\prime}-\Psi^{\prime} \alpha\right)+a_{t}^{\prime(1)} A_{t}^{(3)}-a_{t}^{(1)} A_{t}^{\prime(3)}+\frac{f}{r^{2}}\left(a_{x}^{(1)} A_{x}^{\prime(3)}-A_{x}^{(3)} a_{x}^{\prime(1)}\right)-i \omega a_{t}^{\prime(2)}-\frac{i k f}{r^{2}} a_{x}^{\prime(2)},
\end{aligned}
$$

There are two pure gauge solutions in this sector,

$$
\begin{gathered}
\alpha=0, \quad \beta=i \lambda_{1} \Psi / 2, \quad a_{t}^{(1)}=\lambda_{1} \omega, \quad a_{t}^{(2)}=i \lambda_{1} A_{t}^{(3)}, \quad a_{x}^{(1)}=-\lambda_{1} k, \quad a_{x}^{(2)}=i \lambda_{1} A_{x}^{(3)}, \\
\alpha=i \lambda_{2} \Psi / 2, \quad \beta=0, \quad a_{t}^{(1)}=-i \lambda_{2} A_{t}^{(3)}, \quad a_{t}^{(2)}=\lambda_{2} \omega, \quad a_{x}^{(1)}=-i \lambda_{2} A_{x}^{(3)}, \quad a_{x}^{(2)}=-\lambda_{2} k,
\end{gathered}
$$

where $\lambda_{1}$ and $\lambda_{2}$ are arbitrary constants.

\subsection{Matrix valued Kramers-Kronig relation}

The generically matrix-valued spectral function is defined as

$$
\rho_{i j}(x)=\left\langle\left[\mathcal{O}_{i}(x), \mathcal{O}_{j}(0)\right]\right\rangle,
$$

where $\mathcal{O}_{i}$ are Hermitian operators. Its behavior under Hermitian conjugation is

$$
\rho(x)^{\dagger}=\rho(-x)=-\rho(x)^{t} .
$$

Correspondingly, the Fourier transform $\tilde{\rho}(k)=\int d^{4} x e^{-i k x} \rho(x)$ also satisfies a set of identities

$$
\tilde{\rho}(k)^{\dagger}=\tilde{\rho}(k)=-\tilde{\rho}(-k)^{t} .
$$

In particular this means that the diagonal components are real and antisymmetric under $k \rightarrow-k$. One may also be interested in the behavior under $\omega \rightarrow-\omega$. We take now $k=(\omega, \mathbf{q})$. For theories with rotational invariance the spectral function can depend only on $\mathbf{q}^{2}$. Consequently the diagonal components will also be real and odd in $\omega$

$$
\rho_{i i}\left(\omega, \mathbf{q}^{2}\right)=\rho_{i i}\left(\omega, \mathbf{q}^{2}\right)^{*}=-\rho_{i i}\left(-\omega, \mathbf{q}^{2}\right) .
$$

For the off-diagonal components however, only if one also imposes time reversal or parity symmetry can one prove that the off-diagonal entries must be either even or odd functions of the frequency. In the present case time reversal symmetry is broken by the presence of the chemical potential. Further constraints can however by obtained by supposing that the theory is invariant under $\mathbf{x} \rightarrow-\mathbf{x}$. For an odd number of spatial dimensions we could use the parity operators $P$ to take $\mathbf{x} \rightarrow-\mathbf{x}$. In the two spatial dimensions we study in this paper we can take $P$ to by a rotation by $\pi$ (for an arbitrary even number of spatial dimensions $D=2 n$ we could take the angle $\pi$ for all the rotations in the $i, i+1$-th plane for all $i \leq n)$. This P-operator acts as $P \mathcal{O}_{i}(t, \mathbf{x}) P^{-1}=\sigma_{i} \mathcal{O}_{i}(t,-\mathbf{x})$ with $\sigma_{i}= \pm 1$. In odd spatial dimensions $\sigma_{i}$ is the parity of the operator. In even spatial dimension $\sigma_{i}=-1$ if $\mathcal{O}_{i}$ is the component of a spatial vector. Hence

$$
P\left[\rho_{i j}(t, \mathbf{x})\right]=\sigma_{i} \sigma_{j} \rho_{i j}(t,-\mathbf{x}) .
$$


P-invariance implies $\rho_{i j}(t, \mathbf{x})=\sigma_{i} \sigma_{j} \rho_{i j}(t,-\mathbf{x})$, which for the Fourier transform implies that

$$
\tilde{\rho}_{i j}(\omega, \mathbf{q})=-\sigma_{i} \sigma_{j} \tilde{\rho}_{i j}(-\omega, \mathbf{q})^{*} .
$$

So the off-diagonal entries are either odd or even functions of $\omega$ depending on the signs $\sigma_{i}$. In the case where the fields transform in the same way under the parity operator this means that the real (imaginary) part of the off-diagonal components is an odd (even) function of the frequency.

From the spectral function, as defined in 11.3 .18 we can define two causal propagators, namely the retarded and advanced Green's functions

$$
\begin{aligned}
& G_{R}(x)=-i \Theta(t) \rho(x), \\
& G_{A}(x)=i \Theta(-t) \rho(x),
\end{aligned}
$$

where $x=(t, \mathbf{x})$. Using 11.3 .20$)$, one can prove the following relation among the Fourier transforms of these

$$
\tilde{G}_{R}(k)=\tilde{G}_{R}(-k)^{*}=\tilde{G}_{A}(k)^{\dagger} .
$$

From here, we see that the real (imaginary) part, $\operatorname{Re}\left(G_{R}\right)\left(\operatorname{Im}\left(G_{R}\right)\right)$, is even (odd) under $k \rightarrow-k$. We can compute the Fourier transform of the retarded Green's function, which is given by the convolution of the Fourier transform of the Heaviside step function $\tilde{\Theta}(\omega)$ with the Fourier transform of the spectral function $\tilde{\rho}(k)$,

$$
\tilde{G}_{R}(\omega, \mathbf{q})=-i \int_{-\infty}^{\infty} \tilde{\Theta}(\omega-\mu) \tilde{\rho}(\mu, \mathbf{q}) \frac{d \mu}{2 \pi} .
$$

Using the Fourier transform of the step function

$$
\tilde{\Theta}(\omega)=\frac{i}{\omega+i \epsilon}
$$

and the Sokhatsky-Weierstrass theorem we get

$$
\tilde{G}_{R}(\omega, \mathbf{q})=\mathcal{P} \int_{-\infty}^{\infty} \frac{\tilde{\rho}\left(\omega^{\prime}, \mathbf{q}\right)}{\omega-\omega^{\prime}} \frac{d \omega^{\prime}}{2 \pi}-\frac{i}{2} \tilde{\rho}(\omega, \mathbf{q})
$$

where $\mathcal{P}$ denotes the principle value. From the Hermiticity of $\tilde{\rho}(k)$ we see that we can regard 11.3.28 as a split of $\tilde{G}^{R}(k)$ into its Hermitian and anti-Hermitian parts, and find that the spectral function can be computed from the anti-Hermitian part of the Fourier transform of the retarded Green's function

$$
\tilde{\rho}(k)=i\left[\tilde{G}_{R}(k)-\tilde{G}_{R}(k)^{\dagger}\right] \equiv 2 i \tilde{G}_{R}^{(A)}(k),
$$

where the $(A)$ stands for anti-Hermitian 4 . Plugging this back into 11.3.28 and taking the Hermitian part $(H)$ on both sides we arrive at

$$
\tilde{G}_{R}^{(H)}(\omega)=\frac{i}{\pi} \mathcal{P} \int_{-\infty}^{\infty} \frac{G_{R}^{(A)}\left(\omega^{\prime}\right)}{\omega-\omega^{\prime}} d \omega^{\prime},
$$

which is nothing but the Kramers-Krönig relation for the matrix Green's function. It is complemented by the conjugate relation interchanging the Hermitian and anti-Hermitian

\footnotetext{
${ }^{4}$ Using 11.3 .26 we can always work with retarded Green's functions $G_{R}$.
} 
parts. Imposing P-invariance and using (11.3.23) and 11.3.28 if follows that the Green's function satisfies

$$
\tilde{G}_{i j}^{R}(\omega, \mathbf{q})=\sigma_{i} \sigma_{j} \tilde{G}_{i j}^{R}(-\omega, \mathbf{q})^{*} .
$$

This constrains the QNM spectrum. Taking for example a diagonal Green's function with $i=j$ and writing it as a sum over quasinormal frequencies [46, 153] one seems that the quasinormal frequencies have to come either in pairs obeying $\omega_{n}$ and $\tilde{\omega}_{n}=-\omega_{n}^{*}$ or are confined to lie on the imaginary axis. The residues of the pairs are related by complex conjugation and the purely imaginary ones have to have also purely imaginary residue.

\subsection{Solving the fluctuation equations}

The (1) - (2) sector of the gauged model in the broken phase consists of a system of coupled equations (8.2.70)-8.2.77). In order to extract the spectrum of quasinormal modes we made use of the techniques detailed in [2,51, where a method to compute the poles of the Green functions in terms of non-gauge invariant fields was developed. The quasinormal frequencies are given by the zeroes of the determinant of the field matrix spanned by a maximal set of linearly independent solutions satisfying infalling boundary conditions on the horizon evaluated at the boundary.

Imposing infalling boundary conditions, the near horizon behavior of the fields solving the mentioned equations reads

$$
\begin{aligned}
\alpha & =(\rho-1)^{\kappa}\left(\alpha_{(0)}+\alpha_{(1)}(\rho-1)+\ldots\right), \\
\beta & =(\rho-1)^{\kappa}\left(\beta_{(0)}+\beta_{(1)}(\rho-1)+\ldots\right), \\
a_{t}^{(i)} & =(\rho-1)^{\kappa+1}\left(a_{t(0)}^{(i)}+a_{t(1)}^{(i)}(\rho-1)+\ldots\right), \\
a_{x}^{(i)} & =(\rho-1)^{\kappa}\left(a_{x(0)}^{(i)}+a_{x(1)}^{(i)}(\rho-1)+\ldots\right),
\end{aligned}
$$

where $\kappa=-i \omega / 3$ and $i=1,2$. Since the system is subject to two constraints, we can only choose four of the six parameters at the horizon. Without loss of generality, solutions can be parametrized by $\left\{\alpha_{(0)}, \beta_{(0)}, a_{x(0)}^{(i)}\right\}$. In this way it is possible to construct four independent solutions to the field equations. We can label them as $I, I I, I I I, I V$.

Two additional solutions, $V, V I$, can be obtained by performing gauge transformations of the trivial solution,

$$
\begin{aligned}
& \alpha \rightarrow 0, \beta \rightarrow i \frac{\lambda_{1} \Psi}{2}, a_{x}^{(1)} \rightarrow-k \lambda_{1}, a_{x}^{(2)} \rightarrow 0, a_{t}^{(1)} \rightarrow \omega \lambda_{1}, a_{t}^{(2)} \rightarrow i \Theta \lambda_{1} \\
& \alpha \rightarrow i \frac{\lambda_{2} \Psi}{2}, \beta \rightarrow 0, a_{x}^{(1)} \rightarrow 0, a_{x}^{(2)} \rightarrow-k \lambda_{2}, a_{t}^{(1)} \rightarrow-i \Theta \lambda_{2}, a_{t}^{(2)} \rightarrow \omega \lambda_{2}(
\end{aligned}
$$

where $\lambda_{i}$ are arbitrary constants. Notice that these pure gauge solutions are not algebraic since they have a nontrivial dependence on the bulk coordinate $\rho$.

The most general solution for each field $\varphi_{i}=\left\{\tilde{\alpha}, \tilde{\beta}, a_{t}^{(i)}, a_{x}^{(i)}\right\}$ is given by a linear combination of the above solutions, including the pure gauge modes,

$$
\varphi_{i}=c_{I} \varphi_{i}^{I}+c_{I I} \varphi_{i}^{I I}+c_{I I I} \varphi_{i}^{I I I}+c_{I V} \varphi_{i}^{I V}+c_{V} \varphi_{i}^{V}+c_{V I} \varphi_{i}^{V I},
$$

where we have defined $\{\tilde{\alpha}(\rho), \tilde{\beta}(\rho)\}=\{\rho \alpha(\rho), \rho \beta(\rho)\}$. This convenient choice allows us to identify the asymptotic boundary values $\varphi_{i}$ with the sources of the gauge invariant operators of the dual field theory. 
As shown in [2], the poles of the retarded Green functions will be given by the values of the frequency for which the determinant of the matrix spanned by $\varphi_{i}^{N}$ vanishes asymptotically. Expanding the determinant and evaluating it at a cutoff $\rho=\Lambda$, it reads

$$
\begin{aligned}
& 0=\frac{1}{\lambda_{1} \lambda_{2}} \operatorname{det}\left(\begin{array}{cccccc}
\varphi_{\alpha}{ }^{I} & \varphi_{\alpha}{ }^{I I} & \varphi_{\alpha} I I I & \varphi_{\alpha}{ }^{I V} & \varphi_{\alpha}{ }^{V} & \varphi_{\alpha}{ }^{V I} \\
\varphi_{\beta}{ }^{I} & \varphi_{\beta}{ }^{I I} & \varphi_{\beta} I I & \varphi_{\beta}{ }^{I V} & \varphi_{\beta} V & \varphi_{\beta} V I \\
\varphi_{t(1)}{ }^{I} & \varphi_{t(1)} I I & \varphi_{t(1)} I I & \varphi_{t(1)} I V & \varphi_{t(1)} V & \varphi_{t(1)} V I \\
\varphi_{t(2)} I & \varphi_{t(2)} I I & \varphi_{t(2)} I I & \varphi_{t(2)} I V & \varphi_{t(2)} & \varphi_{t(2)} V I \\
\varphi_{x(1)} I & \varphi_{x(1)} I I & \varphi_{x(1)} I I & \varphi_{x(1)} I & \varphi_{x(1)} V & \varphi_{x(1)} V I \\
\varphi_{x(2)} I & \varphi_{x(2)} I I & \varphi_{x(2)} I I & \varphi_{x(2)} I V & \varphi_{x(2)} V & \varphi_{x(2)} V I
\end{array}\right) \\
& =\omega^{2} \operatorname{det}\left(\begin{array}{cccc}
\varphi_{\alpha}^{I} & \varphi_{\alpha}^{I I} & \varphi_{\alpha}^{I I I} & \varphi_{\alpha}^{I V} \\
\varphi_{\beta}^{I} & \varphi_{\beta}^{I I} & \varphi_{\beta}^{I I I} & \varphi_{\beta}^{I V} \\
\varphi_{x(1)}^{I} & \varphi_{x(1)}^{I I} & \varphi_{x(1)}^{I I I} & \varphi_{x(1)}^{I V} \\
\varphi_{x(2)}^{I} & \varphi_{x(2)}^{I I} & \varphi_{x(2)}^{I I I} & \varphi_{x(2)}^{I V}
\end{array}\right)+\omega k \operatorname{det}\left(\begin{array}{cccc}
\varphi_{\alpha}^{I} & \varphi_{\alpha}^{I I} & \varphi_{\alpha}^{I I I} & \varphi_{\alpha}^{I V} \\
\varphi_{\beta}^{I} & \varphi_{\beta}^{I I} & \varphi_{\beta}^{I I} & \varphi_{\beta}^{I V} \\
\varphi_{t(1)}^{I I} & \varphi_{t(1)}^{I I} & \varphi_{t(1)}^{I I I} & \varphi_{t(1)}^{I V} \\
\varphi_{x(2)}^{I} & \varphi_{x(2)}^{I I} & \varphi_{x(2)}^{I I I} & \varphi_{x(2)}^{I V}
\end{array}\right) \\
& -\omega k \operatorname{det}\left(\begin{array}{cccc}
\varphi_{\alpha}^{I} & \varphi_{\alpha}^{I I} & \varphi_{\alpha}^{I I I} & \varphi_{\alpha}^{I V} \\
\varphi_{\beta}^{I} & \varphi_{\beta}^{I I} & \varphi_{\beta}^{I I I} & \varphi_{\beta}^{I V} \\
\varphi_{t(2)}^{I I} & \varphi_{t(2)}^{I I} & \varphi_{t(2)}^{I I I} & \varphi_{t(2)}^{I V} \\
\varphi_{x(1)}^{I} & \varphi_{x(1)}^{I I} & \varphi_{x(1)}^{I I I} & \varphi_{x(1)}^{I V}
\end{array}\right)+k^{2} \operatorname{det}\left(\begin{array}{cccc}
\varphi_{\alpha}^{I} & \varphi_{\alpha}^{I I} & \varphi_{\alpha}^{I I I} & \varphi_{\alpha}^{I V} \\
\varphi_{\beta}^{I} & \varphi_{\beta}^{I I} & \varphi_{\beta}^{I I I} & \varphi_{\beta}^{I V} \\
\varphi_{t(1)}^{I V} & \varphi_{t(1)}^{I I} & \varphi_{t(1)}^{I I I} & \varphi_{t(1)}^{I V} \\
\varphi_{t(2)}^{I} & \varphi_{t(2)}^{I I} & \varphi_{t(2)}^{I I I} & \varphi_{t(2)}^{I V}
\end{array}\right)
\end{aligned}
$$

where the background boundary conditions $\Theta(\Lambda)=0$ and $\Lambda \Psi=0$ have been already imposed. This absence of background sources for the corresponding operators makes the point $(\omega, k)=(0,0)$ a trivial solution to the vanishing determinant condition, which ensures the existence of a hydrodynamic mode. Notice also that the point $(\omega, k)=(0,0)$ is a double solution to the previous determinant equation.

Solutions to the equations of motion and to the determinant condition $(11.4 .39)$ have been computed numerically. It has been checked that the election of solution basis, i.e. of initial values of the free parameters, does not affect the result. 
Part IV

\section{Anomalous transport Phenomena and superfluids}




\section{Chapter 12}

\section{Chiral transport in Holographic Superfluids}

It could be stated that the study of the interplay between anomalous transport and superfluids started a decade ago; the first approaches to chiral transport (concretely, the Chiral Separation Effect) where analyzed for high-density QCD, assuming for instance that baryon symmetry is spontaneously broken, see [58,63]. However, a systematic study of Chiral Superfluids has only been undertaken recently, using different techniques to obtain the hydrodynamic expansion, with particular emphasis on the anomalous response 96, 124,199 201.

The results indicate that the effect of the background condensate is two-fold. On the one hand, unlike the case of ordinary fluids (see Chapter 3 for an introduction), anomalous conductivities are not fully determined by anomaly coefficients anymore. On the other, in addition to the Chiral Vortical and Chiral Magnetic effects, there exist new types of transport phenomena driven by the anomalies. However, until now, we lack clear predictions for the anomalous response parameters in superfluids.

Remarkably, it has been recently pointed out that, for a certain class of holographic models of chiral superfluids [202] the zero-temperature behaviour of the CMC and CVC is universal and given by [4]

$$
\begin{aligned}
& \sigma_{55}^{\text {brok. }}(T \rightarrow 0)=\frac{\sigma_{55}^{\text {unbrok. }}}{3}, \\
& \sigma_{C V C}^{\text {brok. }}(T \rightarrow 0)=0,
\end{aligned}
$$

where "brok." and "unbrok." refer to broken and unbroken phases, respectively.

Here we address the possible corrections of the anomalous transport coefficients due to the presence of condensates, performing an explicit computation of them. We will focus on the strongly coupled regime and, to simplify the approach, we will stick to s-wave condensates. To that end, we use holographic methods.

Contrary to the usual approaches to transport in Chiral Superfluids, here we will rely on linear response theory to analyze the possible corrections. Kubo formulae (see the introduction) provide us with the response driven by a small external perturbation. These are powerful because they account automatically for all the corrections to the coefficients and sometimes prove the existence of new transport phenomena which is difficult to analyze by means of hydrodynamic expansions. Hence, we assume that it is possible to define the anomalous conductivities in terms of correlators in the broken phase, which is to say, that there exists a current due to an external magnetic field in both the unbroken and broken 
phase: $4^{1}$

$$
\begin{aligned}
\mathcal{J}^{i} & =\sigma_{\{C M E, C S E, 55\}} B^{i}, \\
\mathcal{J}^{i} & =\sigma_{C E E} \epsilon^{i j} E_{j} .
\end{aligned}
$$

Where $\mathcal{J}, B, E$ correspond to a generic $U(1)$ covariant current (defined by equation (3.0.16)), magnetic and electric field, respectively, whereas $\sigma$ denotes generic conductivities 2 , Equation (12.0.4) represents the Chiral Electric Effect (CEE), an anomalous transport phenomenon which is present only for Chiral Superfluids at finite superfluid velocity, see [124. We will propose a Kubo formula for the Chiral Electric Conductivity (CEC) and compute its value in our models.

In addition, we emphasize the existence of a type of transport phenomena in Chiral Superfluids that to our knowledge has been overlooked so far. We will call it Chiral Charge Generation Effect (CCGE). It establishes the presence of a charge density whenever the supervelocity is aligned with an external magnetic field

$$
\rho=\hat{\sigma} \vec{\xi} \cdot \vec{B}
$$

here $\vec{\xi}$ is the superfluid velocity and $\hat{\sigma}$ the corresponding conductivity (CCGC). We will provide a Kubo formula for it in Section 12 and compute its value, showing that it is generically different from zero. The response prescribed by 12.0 .5 is not formally new, even though we believe its physical importance has not been stressed before. It has appeared in the literature and for instance it can be mapped to the term $S_{1}$ of equation (2.31) of 96$]^{3}$. Such a term establishes the presence of a charge density whenever a transverse London-type-current $S_{1}=\epsilon^{i j k} \zeta_{i} \partial_{j} \zeta_{k}$ is acting on the system. Since $\zeta_{k}=-\partial_{k} \phi+A_{k}$ (see [96]) we propose that there is an effective response of the form (12.0.5) arising from $S_{1}=\epsilon^{i j k} \zeta_{i}^{0} \partial_{j} A_{k}+\ldots$. We believe that such a transport phenomenon leads to interesting phenomenological implications.

Notice that, for the above formulae to make sense, it is important in general that the background we are considering is stable in the presence of a (perturbatively small) magnetic field, i.e. that there exists a perturbative expansion in the amplitude of a external magnetic field. Given such a perturbative expansion, at zeroth order the holographic superfluid corresponds to the background considered here. This is consistent with the usage of Kubo Formulae to compute the transport coefficients. However, for finite external magnetic fields, the holographic superfluid gets affected and, in particular, it generates London-type currents 3 . Therefore, one could argue against the validity of our results beyond perturbatively small external sources. In order to avoid that potential issue, in Section 12.2 we study a $U(1) \times U(1)$ model, in which only one of the $U(1)$ 's undergoes a phase transition and thus we can study how the (unscreened) magnetic field associated to the unbroken symmetry enters the chiral transport properties.

In what follows we will work with global symmetries in the QFT, for they are very naturally accommodated within holography. This means that we can restrict ourselves to configurations which do not excite the anomaly. This is a pertinent remark, since having a dynamical photon would imply the existence of general loop corrections to the anomalous transport coefficients [123] which are important even in the hydrodynamic approximation.

\footnotetext{
${ }^{1}$ For a detailed analysis of some of the Kubo formulae applied to Chiral Superfluids, see 203 .

${ }^{2}$ CSE stands for Chiral Separation Effect.

${ }^{3}$ We thank Carlos Hoyos for pointing this out.
} 
Despite the fact that there is no photon here, in the broken phase the Goldstone boson could in general give important corrections at strong coupling. However, we expect our calculation not to capture all these contributions, for they are subleading in the classical gravity approximation.

A source of the corrections that we should be able to capture within holography is the one associated to the background scalar field. For instance, in 204 the Chiral Separation Conductivity (CSC) was indeed found to present corrections in the case of a linear sigma model (the background scalar field gives an effective mass to the fermions through the Yukawa coupling and contributes to the CSC).

Entropic arguments were used in [124] to extract the Hydrodynamics of Chiral Superfluids in the presence of external unbroken gauge fields. The Chiral Electric Effect was predicted and some possible generic corrections to the CMC and CVC were found. Moreover, in [4] it was argued that such corrections do not vanish but become universal (model independent) at low temperatures and the CMC and the CVC were computed at $T=0$, indeed finding a universal result. Our models are restricted to the probe limit and hence we will not be able to reach $T \rightarrow 0$; furthermore, we cannot induce metric perturbations and hence the CVC cannot be calculated. However, we observe that the chiral conductivities stabilize fast enough to be able to observe their $T=0$ behaviour even at temperatures close enough to $T_{c}$ where our computations are reliable.

In what follows we consider two models, one in which a $U(1)$ anomalous symmetry undergoes a phase transition and one in which we have two $U(1)$ symmetries and just one of them develops a condensate. In the absence of supervelocity the former case reduces to a truncation of the model of [4] and indeed we observe that $\sigma_{55}$ approaches the value prescribed by equation (12.0.1). In the latter model (not considered so far in the literature) at zero supervelocity we can define three non-vanishing anomalous conductivities 135; our results suggest that all of them approach universal values at low temperatures. Remarkably enough, the universal ratio is always different from $1 / 3$ and, in particular, the $\mathrm{CMC}$ vanishes as we increase the chemical potential.

\section{Remarks on the definition of the current}

At this point it is important to point out several remarks related to the definition of the currents. In principle, one could use the consistent currents to define the anomalous correlators. As pointed out in 205], one has to be careful in this case, for the gauge fields at infinity are not directly related to the chemical potential of the theory. In [135] an holographic calculation of the anomalous transport coefficients, taking the previous issue into account, was carried out; it was shown how one has to give up the condition that the background gauge field vanishes at the horizon in order to be able to distinguish the source from the chemical potential.

In the presence of a condensate, regularity imposes that the gauge field must be zero at the horizon. Hence, it is better to work from the start with the covariant definition of the current, as in Chapter 6. Notice that this amounts to neglecting the contribution to the current operator coming from the holographic Chern-Simons term. With this manipulation there is no trace of the sources in the correlators and one can perfectly work with a boundary condition such that the background gauge field vanishes at the horizon. The resulting correlators are the ones of [135] with $\alpha=\beta=0$. Physically, we thus will be working with the covariant current, and our computed retarded two-point functions 
contain therefore one covariant and one consistent current, namely

$$
\mathcal{G}_{\mathcal{R}} \sim\left\langle\mathcal{J}^{\text {cov }} \mathcal{J}^{\text {cons }}\right\rangle
$$

Notice that this in particular implies that, no matter the model under consideration, none of our (covariant) currents is conserved in general. However, this is not a problem at all since our background gauge field configurations are such that the anomaly is not excited.

\section{Remarks on the Kubo Formulae}

Let us point out some remarks on the Kubo formulae we are going to use. We lack formal derivation of the one corresponding to the CEE. However, assuming a constitutive relation of the form (12.0.4), we can derive a suitable Kubo formula for it. We point out that we do not intend to make contact with the hydrodynamic construction of [124](for example, our Kubo relations are associated to the laboratory frame, not the Landau frame). Instead, we will propose suitable Kubo formulae for the conductivities we aim to study, based on the fact that we know which the gauge-invariant sources are, as well as the type of response that we expect. Our Kubo formulae read

$$
\begin{aligned}
\sigma_{\{55, C S E, C M E\}} & =\lim _{k \rightarrow 0} \frac{i}{2 k}\left\langle J^{y} J^{z}\right\rangle_{\mathcal{R}}(\omega=0, k), \\
\sigma_{C E C} & =\lim _{k \rightarrow 0} \frac{i}{2 \omega}\left\langle J^{y} J^{z}\right\rangle_{\mathcal{R}}(\omega, k=0), \\
\sigma_{C C G E} & =\lim _{k \rightarrow 0} \frac{i}{2 k_{\perp}}\left\langle J^{0} J^{y}\right\rangle_{\mathcal{R}}(\omega=0, k) .
\end{aligned}
$$

Where $k_{\perp}$ means that the momentum points in a direction transverse to the supervelocity. All the conductivities in (12.0.7) are associated to similar correlators. The distinction between them comes from the nature of the currents inside the two point functions and it only makes sense in the presence of more than one $U(1)$. This will be made explicit in Section 12.2 . We believe the above provide suitable expressions due to the following

- All the above conductivities vanish in the absence of anomaly.

- For $\sigma_{\{55 ; C S E ; C M E\}}$ we rely on the fact that they are related to the response to an external magnetic field by definition. Moreover, as we will see, (12.0.7) is continuous through the phase transition, matching the value that $\sigma_{\{55, C S E, C M E\}}$ shows in the unbroken phase. In addition to this, our formula coincides with the one of 203.

- In the case of $\sigma_{C E C}$, we take into account that it corresponds to the effect of an external electric field, as in [124]. With this in mind, we choose a kinematic limit such that it can be drastically distinguished from the other anomalous transport coefficients. Moreover, we will observe that $\sigma_{C E C} \sim \xi$ at low temperatures.

- The formula $(12.0 .9)$ can be derived from the discussion of 203 (our notation is also taken from that reference). We start with the term $J^{0}=-T_{0} e^{\sigma} g_{1, \nu} S_{1}{ }^{4}$ and take the variation

$$
\frac{\delta S_{1}}{\delta A_{l}}=\left.2 i k_{j} \epsilon^{i j k} \zeta_{i}^{e q} \cdot \frac{\delta \zeta_{k}^{e q .}}{\delta A_{l}}\right|_{\text {sources }=0}
$$

\footnotetext{
${ }^{4} g_{1, \nu}$ is the derivative of the thermal coefficient $g_{1}$ with respect to $\nu \equiv \mu / T \quad 96$
} 
where the 2 comes from the fact that we have twice the same contribution $\epsilon^{i j k} \zeta_{i}^{e q \cdot} \partial_{j} \frac{\delta \zeta_{k}^{e q .}}{\delta A_{l}}$. For transverse momentum, we use equation (3.29) of 203, yielding

$$
\begin{aligned}
\frac{\delta \zeta_{i}^{e q .}}{\delta A_{l}} & =\delta_{i}^{l}-\frac{k_{i} k^{l}}{k^{2}}-2 i T_{0} c_{3} k_{i} \zeta_{0}^{l}, \\
\frac{\delta S_{1}}{\delta A_{l}} & =2 i k_{j} \epsilon^{i j k} \zeta_{i}^{0}\left(\delta_{k}^{l}-\frac{k_{k} k^{l}}{k^{2}}-2 i T_{0} c_{3} k_{k} \zeta_{0}^{l}\right) .
\end{aligned}
$$

Now, $k_{k} k_{j} \epsilon^{k j}=0$ and hence, to first order in $k$ we have

$$
\left\langle J^{0} J^{l}\right\rangle=-2 i T g_{1, \nu} k_{j} \epsilon^{i j l} \zeta_{i}+\mathcal{O}\left(k^{2}\right)
$$

where all the equilibrium super/subscripts "0" have been omitted. From here,

$$
\epsilon_{l m n} \mathcal{G}_{R}^{0 l}=-2 i T g_{1, \nu} k_{j} \zeta_{i}\left(\delta_{m}^{i} \delta_{n}^{j}-\delta_{m}^{j} \delta_{n}^{i}\right)
$$

where $\mathcal{G}_{R}^{0 l} \equiv\left\langle J^{0} J^{l}\right\rangle$. The formula 12.0 .5 can be recovered by assuming $m=z, n=$ $x$. In our notation $\zeta_{i} \equiv \xi_{i}$ and we get

$$
\sigma_{C C G E} \equiv T \xi_{z} g_{1, \nu}=\lim _{k_{x} \rightarrow 0} \frac{i}{2 k_{x}} \mathcal{G}_{R}^{0 y}(\omega=0)
$$

To avoid any possible confusion let us point out that, taking advantage of the fact that we work with a fixed component of the supervelocity, throughout this work we will usually absorb the supervelocity factors into the conductivities, as prescribed by equations 12.0.8) and (12.0.9). This can be seen explicitly in 12.0.15). Of course, in general one has to take into account that the CEE and CCGE are linear in the supervelocity (a vector) and write expressions like 12.0.5 instead.

\subsection{Broken Anomalous symmetry}

We want to analyze, from the holographic point of view, how the anomalous conductivities are altered due to the presence of an s-wave condensate. To this end we consider a holographic superconductor plus a Chern-Simons term that induces a $U(1)^{3}$ anomaly in the dual field theory.

From the point of view of the dual field theory we have a spontaneously broken $U(1)$ anomalous global symmetry. The action of the bottom-up model reads

$$
S=\int d^{5} x \sqrt{-g}\left(-\frac{1}{4} F_{M N} F^{M N}+\frac{\kappa}{3} \epsilon^{M A B C D} A_{M} F_{A B} F_{C D}-\overline{D_{M} \Psi} D^{M} \Psi-m^{2} \bar{\Psi} \Psi\right)
$$

This is the model of [4] with $V_{\psi}=1, V=m^{2} \bar{\Psi} \Psi$ and $\kappa=c / 8$. In what follows we will be working with the covariant definition of the current, meaning that we are neglecting the Chern-Simons contribution to the definition of $J^{\mu}$.

We take the Schwarzschild AdS Black Brane in 5 dimensions as our background metric in the bulk

$$
d s^{2}=-f(r) d t^{2}+\frac{d r^{2}}{f(r)}+\frac{r^{2}}{L^{2}}\left(d x^{2}+d y^{2}+d z^{2}\right)
$$


being $f(r)=\frac{r^{2}}{L^{2}}-\frac{r_{H}^{2}}{r^{2}}$. From now on we will work in adimensional units, rescaling all the $L^{2}$ factors to one. Our ansatz for the background fields consists of a non-vanishing temporal and spatial component of the gauge field and the real component of the scalar field. All of them with just radial dependence

$$
A=\phi(r) d t+V(r) d x ; \quad \Psi(r)=\psi(r)
$$

With this ansatz the background equations of motion reduce to

$$
\begin{aligned}
\phi^{\prime \prime}+\frac{3}{r} \phi^{\prime}-\frac{2 \psi^{2}}{f} \phi & =0 \\
\psi^{\prime \prime}+\left(\frac{f^{\prime}}{f}+\frac{3}{r}\right) \psi^{\prime}+\frac{\phi^{2}}{f^{2}} \psi-\frac{V^{2}}{r^{2} f} \psi-\frac{m^{2}}{f} \psi & =0 \\
V^{\prime \prime}+\left(\frac{f^{\prime}}{f}+\frac{1}{r}\right) V^{\prime}-\frac{2 \psi^{2}}{f} V & =0
\end{aligned}
$$

The equations boil down to the ones which govern the usual s-wave holographic superconductor in the presence of supervelocity (i.e. equations appeared in Chapter 9p. This could have been anticipated by noticing that the ansatz does not excite the Chern-Simons contribution $\kappa \epsilon^{M A B C D} F_{A B} F_{C D}$ to the gauge field equation, as expected. Hence, the anomaly is absent at the level of the background. However, it has important implications for the perturbations.

In our convention we choose to fix the temperature and interpret the adimensional quan-
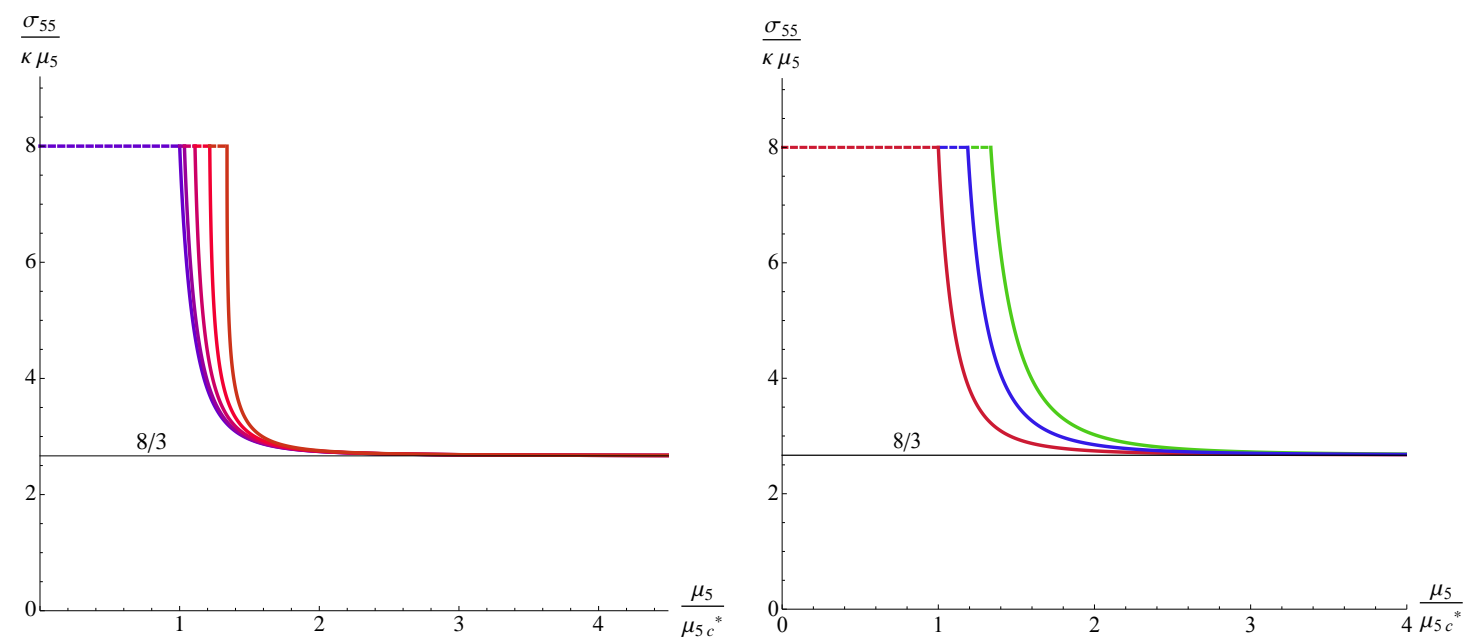

Figure 12.1: Axial conductivity divided by the chemical potential and the anomaly coefficient versus chemical potential. $\mu_{5 c}^{*}$ is the critical chemical potential at zero supervelocity and $m^{2}=-7 / 2$. (Left) Each line corresponds to a different superfluid velocity, from $\xi_{x} / T=0.1$ (blue) to $\xi_{x} / T=2.1$ (orange). The dashed horizontal line corresponds to the unbroken phase, where $\sigma_{55} \sim \mu_{5}$. In the broken phase this conductivity approaches $1 / 3$ of the unbroken phase value for large enough chemical potential. This is compatible with the results of [4]. (Right) Each line corresponds to a different value for the mass (red $m^{2}=-7 / 2$, blue $m^{2}=-3$, green $m^{2}=-5 / 2$ ) of the scalar field in the bulk. As one can see the $1 / 3$ factor is unaltered by the dimension of the operator that condenses. The conductivity depends linearly with $\kappa$.

tities

$$
\bar{\mu}=\frac{\mu}{T} ; \quad \overrightarrow{\vec{\xi}}=\frac{\vec{\xi}}{T}
$$


as the chemical potential and the supervelocity of the system (along this work we make some abuse of language and refer to the $\bar{\mu} \rightarrow \infty$ regime as the $T \rightarrow 0$ limit), which are determined by the boundary conditions of the fields to be imposed at spatial infinity:

$$
\phi(r)_{r \rightarrow \infty} \sim \mu_{5}
$$

By $\xi_{\{x, z\}}$ we mean that the supervelocity will be taken to be pointing either in the $x$ or the $z$-direction. In addition, we choose the standard quantization, by imposing the boundary conditions to the leading term in the asymtotic expansion of the scalar field

$$
\begin{aligned}
& \psi(r)_{r \rightarrow \infty} \sim \frac{\psi_{1}}{r^{\Delta_{-}}}+\frac{\psi_{2}}{r^{\Delta_{+}}}+\ldots \\
& \psi_{1}=0 \quad \psi_{2}=\langle O\rangle
\end{aligned}
$$

We solve equations (12.1.19)-12.1.21) with this boundary conditions numerically.

Before we proceed to discuss our results for the conductivities a comment is in order regarding the background we have constructed. In Section 9.0.9 we argued that the system presents instabilities at finite momentum close to the phase transition for a large range of supervelocities. The stable background in that region is not known. Although this analysis was made in $A d S_{3+1}$ we expect it to apply in $A d S_{4+1}$ as well. We do not discard those issues to have some influence, even though, as we will see later on, all of our results seem to be perfectly consistent for every value of the chemical potential. In any case, let us emphasize that our forthcoming main observations have to do with the behaviour of the conductivities far from the transition point, where the above potential issues are not expected to play any role.

\subsubsection{The Chiral conductivities in the broken phase: Axial conductiv- ity and CEC}

In order to compute the chiral conductivities from the Kubo formulae $12.0 .3-12.0 .5$ we study perturbations on top of the background we have built. We first want to explore the axial conductivity $5^{5}$ and the CEC, therefore we switch on the perturbations with nonvanishing frequency and momentum pointing in the direction parallel to the supervelocity (that we choose to be the $x$-direction). The sector we are interested in decouples from the rest of the field perturbations in this kinematic setup, leaving us with just the perturbations of the transverse gauge fields

$$
\delta A_{y}=a_{y}(r, t, x) ; \quad \delta A_{z}=a_{z}(r, t, x)
$$

\footnotetext{
${ }^{5}$ In some of the literature this conductivity has often been directly associated to the CMC, for the qualitative dependence of the three conductivities of 12.0 .3 on the axial/vector chemical potentials is the same in the absence of condensate. However, there are significant differences when a condensate distinguishing between axial and vector currents is present, as we will see. Thus, we will stick to the notation of 135] and denote as CMC the conductivity related to a vector-vector correlator when a AVV anomaly is switched on.
} 

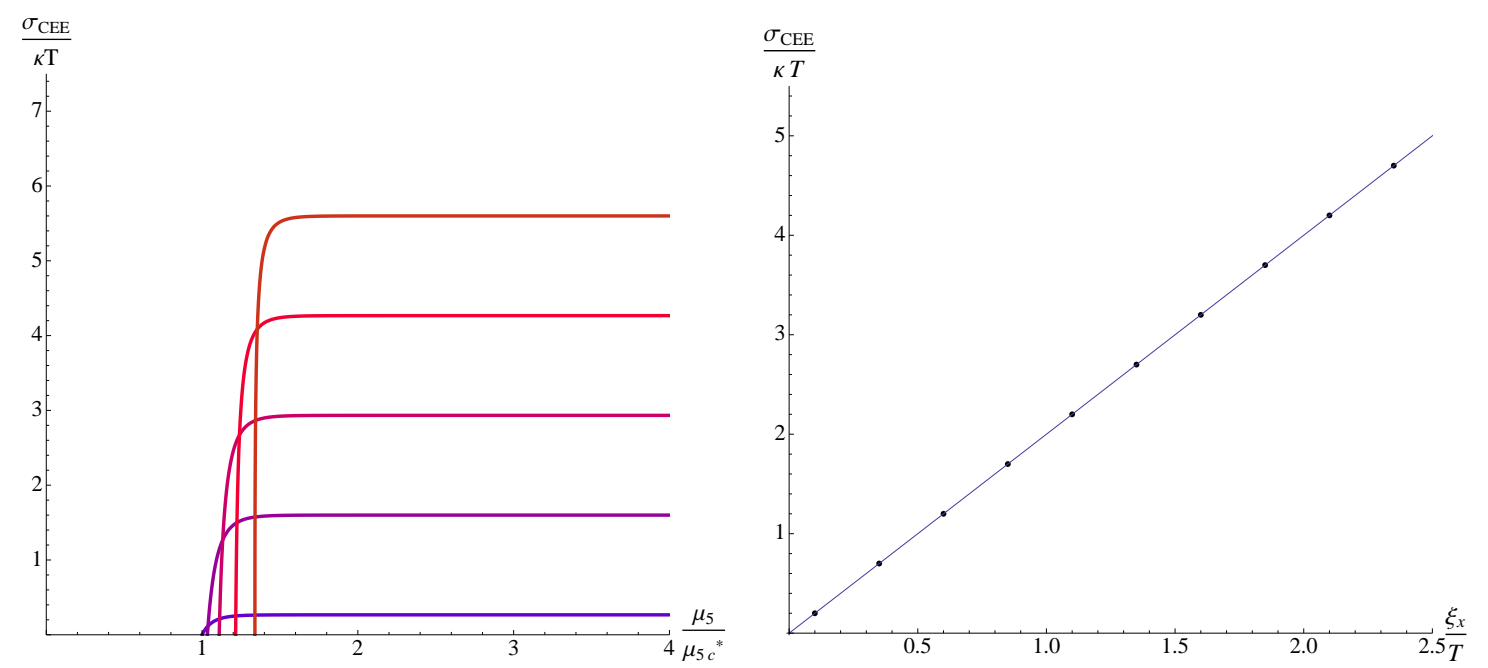

Figure 12.2: (Left) Chiral electric conductivity versus chemical potential. Each line corresponds to a different superfluid velocity, $\xi_{x} / T=0.1-2.1$. We observe that $\sigma_{C E E} / T=0$ at $\mu_{5 c}$ and it approaches a constant value at low temperatures/ large chemical potential. (Right) Dots correspond to $\sigma_{C E E} / \kappa T$ versus $\bar{\xi}_{x}$ in the region in which $\sigma_{C E E} / T$ is independent of $\mu_{5}$. The solid line corresponds to a linear fit; the slope is 2.667. The conductivity depends linearly with $\kappa$.

In momentum space the equations read

$$
\begin{aligned}
& a_{y}^{\prime \prime}+\left(\frac{f^{\prime}}{f}+\frac{1}{r}\right) a_{y}^{\prime}+\frac{1}{f}\left(\frac{\omega^{2}}{f}-\frac{k^{2} L^{2}}{r^{2}}-2 \psi^{2}\right) a_{y}+16 i k \frac{\kappa L}{r f} \phi^{\prime} a_{z}+16 i \omega \frac{\kappa L}{r f} V^{\prime} a_{z}=0 \\
& a_{z}^{\prime \prime}+\left(\frac{f^{\prime}}{f}+\frac{1}{r}\right) a_{z}^{\prime}+\frac{1}{f}\left(\frac{\omega^{2}}{f}-\frac{k^{2} L^{2}}{r^{2}}-2 \psi^{2}\right) a_{z}-16 i k \frac{\kappa L}{r f} \phi^{\prime} a_{y}-16 i \omega \frac{\kappa L}{r f} V^{\prime} a_{y}=0
\end{aligned}
$$

In the unbroken phase it is possible to find an analytic solution to the above system of equations in the kinematic limit $\omega=0$ and to first order in momentum $k_{x} \equiv k$. Recall that this is all that we need in order to compute the $\sigma_{55}$, making use of Kubo formulae 69]. However, in the case at hand the background has been computed numerically and therefore we will look directly for numerical solutions to the system $(12.1 .26)-(12.1 .27)$.

We are now ready to compute the Kubo formulae shown in 112.0 .3$)-(12.0 .5)$. The problem reduces to numerically compute the two retarded 2-point functions with the usual holographic prescription 51](see the Appendix for details on the computation).

A comment that applies to all figures is in order here. The critical value of the chemical potential depends on the value of the supervelocity and the mass of the scalar field. In our convention, $\mu_{c}^{*}$ is the critical value at zero supervelocity and $m^{2}=-7 / 2$.

Our results for $\sigma_{55}$ are depicted in Figure 12.1. We observe that $\sigma_{55}$ is proportional to the (axial) chemical potential even in the broken phase. However, the coefficient of proportionality decreases from 1 to $1 / 3$ in units of $e^{2} N_{c} / 4 \pi^{2}$. Numerically, in terms of $\kappa$ 

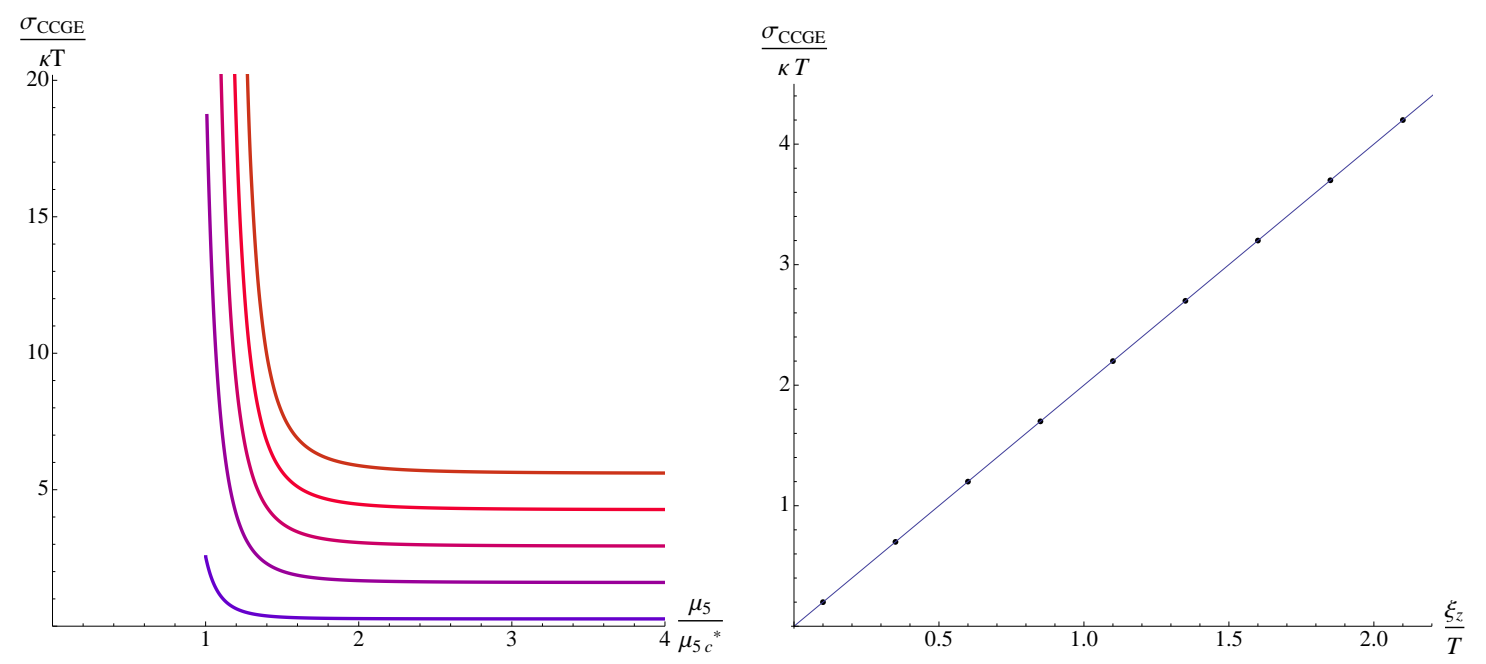

Figure 12.3: (Left) Chiral charge generation conductivity versus chemical potential, different lines correspond to different values of the supervelocity, $\xi_{x} / T=0.1-2.1$. (Right) Dots correspond to $\sigma_{C^{2} G E}$ versus supervelocity for large values of the chemical potential. The solid line corresponds to a linear fit; the slope is 2.667 . The conductivity depends linearly with $\kappa$.

we get 6

$$
\frac{\sigma_{55}\left(\frac{\bar{\mu}_{5}}{\bar{\mu}_{5 c}}>>1\right)}{\kappa \mu_{5}}=2.668 \approx \frac{8}{3} .
$$

This reduction has been predicted to be universal. In our model, we can check that this is independent from the mass of the bulk scalar field (right plot of Figure 12.1). Remarkably, finite supervelocity does not alter these conclusions, as depicted in Figure 12.1 (left); the correction to the transport coefficient is independent of the supervelocity. As a final remark, we find that the dependence of the axial conductivity with $\kappa$ is unaffected by the presence of the condensate and the supervelocity, namely $\sigma_{55} \sim \kappa$.

Moving to the CEC, we observe that it starts increasing but rapidly approaches a constant value, independent of $\mu_{5} / T$. On the contrary, it linearly increases with the superfluid velocity for large chemical potential, see Figure 12.2. Or results thus strongly suggest that, at low temperatures,

$$
\frac{\sigma_{C E E}\left(\frac{\bar{\mu}_{5}}{\bar{\mu}_{5 c}}>>1\right)}{\kappa T}=2.667 \frac{\xi_{x}}{T} \approx \frac{8}{3} \frac{\xi_{x}}{T} .
$$

Notice that this value is essentially the same as the observed for $\sigma_{55}$ at large axial chemical potential. Again the dependence with $\kappa$ is linear.

\subsubsection{The Chiral Charge Generation Effect}

Let us now induce a supervelocity in the $z$-direction, by turning on $A_{z}(r)$ instead of $A_{x}(r)$ in the bulk. This, as anticipated, influences the quasinormal modes, even though the

\footnotetext{
${ }^{6}$ In order to make contact with the computation in the unbroken phase of 69 , notice that we have set $16 \pi G \equiv 1$ in 12.1 .16 . Hence, their result $\sigma_{B}^{\text {unbrok. }}=8 \kappa \mu_{5} /(16 \pi G)$ corresponds to $\sigma_{55}^{\text {unbrok. }}=8 \mu_{5} \kappa$ with our conventions.
} 
background equations remain the same as in the previous subsection (due to the fact that, without superflow, the background is isotropic), with the replacement $A_{x} \leftrightarrow A_{z}$. On top of this we switch on the perturbations with non-vanishing frequency and momentum pointing in the $x$-direction (transverse to the supervelocity). The equations for the perturbations in the transverse sector are more involved now for they couple to all other perturbations. They can be found in Appendix 12.5.1.

As mentioned in the introduction, the CCGE corresponds to a "generation" of charge proportional to the scalar product of the supervelocity and the magnetic field

$$
\rho=\hat{\sigma} \vec{\xi} \cdot \vec{B} .
$$

As aforementioned, for convenience we will absorb the supervelocity component into the conductivity, i.e. $\sigma_{C C G E}=\hat{\sigma} \xi_{z}$. Note that the charge vanishes if the supervelocity is parallel to the external momentum. In order to observe such an effect, we will use (12.0.9). We proceed as before and present our result in Figure 12.3. We observe that indeed this phenomenon is not negligible in the presence of supervelocity. Moreover, it stabilizes at large enough chemical potential; in the region in which $\sigma_{C C G E}$ does not depend on $\bar{\mu}_{5}$, it presents a clear linear dependence on the superfluid velocity (right plot of Figure 12.3). We can perform a numerical quadratic fit, obtaining

$$
\frac{\sigma_{C C G E}\left(\frac{\bar{\mu}_{5}}{\bar{\mu}_{5 c}}>>1\right)}{\kappa T}=2.667 \frac{\xi_{z}}{T} \approx \frac{8}{3} \frac{\xi_{z}}{T}
$$

to a good approximation. Again, the slope has the same value as for the CEC. Let us emphasize that the behaviour of this transport coefficient at the phase transition is strange at first sight. Naively, we would have expected $\sigma_{C C G E}\left(\bar{\mu}_{c}\right)=0$ instead of the observed value. We comment on this issue in Section 12.3 .

\subsection{Model with axial and vector currents}
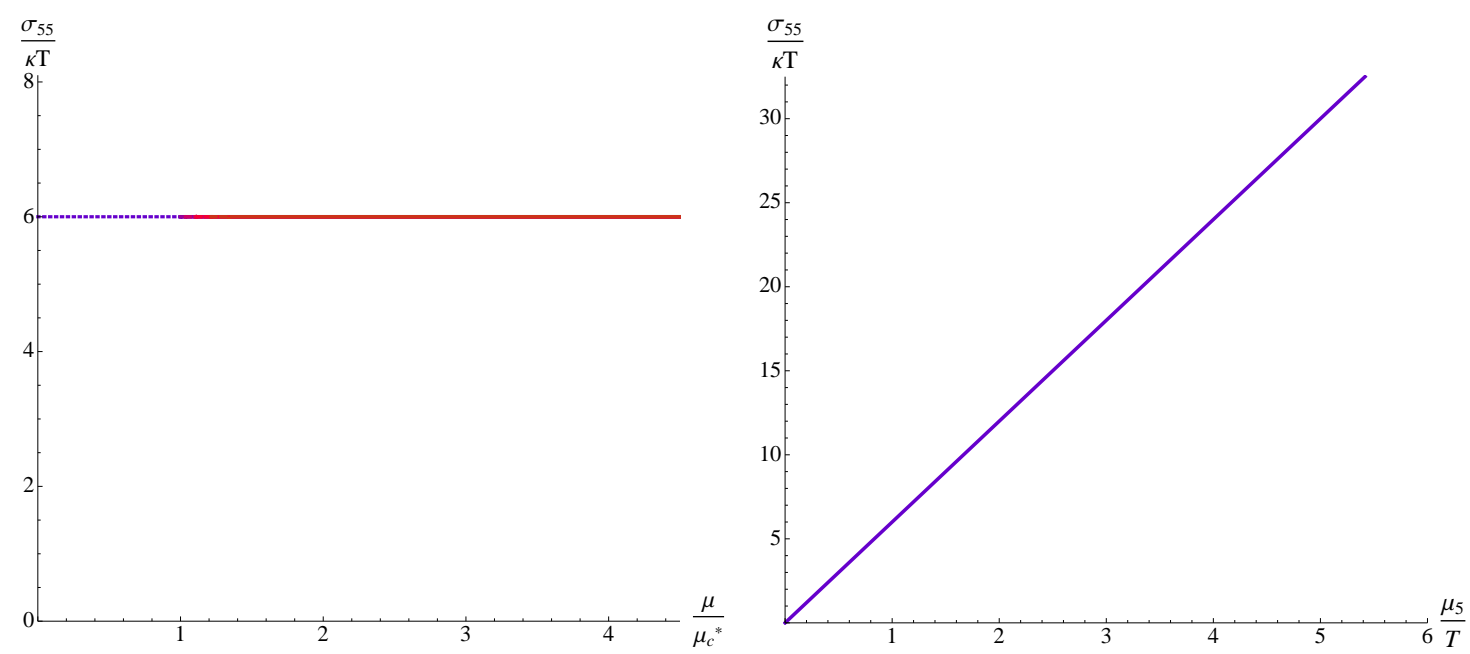

Figure 12.4: (Left) Axial conductivity versus vector chemical potential at $\bar{\mu}_{5}=1$ and $\xi_{x} / T=0.1-2.1$. We find that $\sigma_{55}$ is independent of both the vector chemical potential and the superfluid velocity. (Right) $\sigma_{55}$ versus axial chemical potential. The dependence is linear, as expected. The conductivity depends linearly with $\kappa$. 

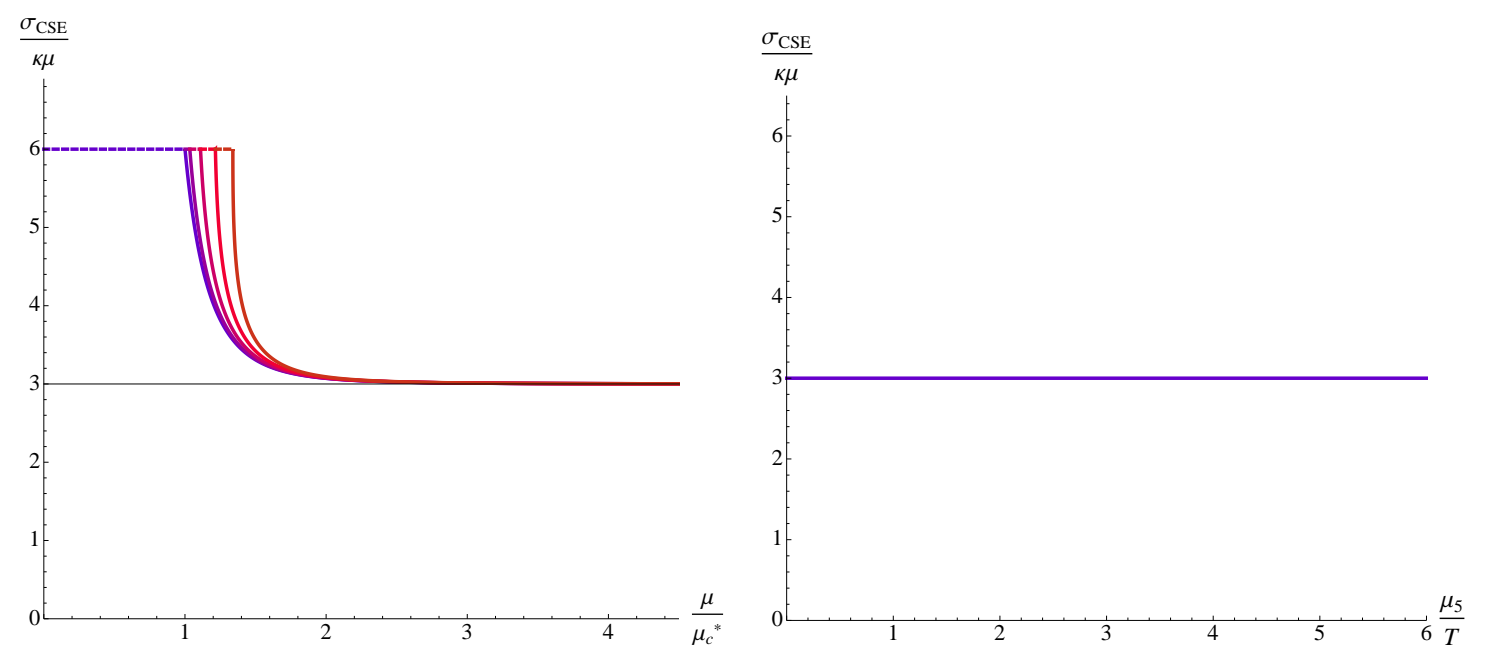

Figure 12.5: (Left) Chiral separation conductivity divided by vector chemical potential versus vector chemical potential, $\bar{\mu}_{5}=1$ and $\xi_{x} / T=0.1-2.1$. The conductivity now approaches $1 / 2$ of the value at $\bar{\mu}_{c}$, independently of $\xi_{x} / T$. (Right) The plot shows this conductivity against the axial chemical potential for generic values of $\mu$. $\sigma_{C S E}$ is independent of the axial chemical potential in both the broken and ubroken phases. The conductivity depends linearly with $\kappa$.

In this section we study the more realistic model, in which we consider two $U(1)$ bulk gauge fields, being only one of them spontaneously broken. There are two different interpretations of this model:

- We have axial and vector currents $U(1)_{V} \times U(1)_{A}$ and the condensate is coupled only to the vector part, whereas the axial symmetry is unbroken. This realizes the interplay between anomalous axial and vector currents, first considered in [135].

The fact that the axial current is not coupled to the scalar field means that the axial charge of the condensate is zero, so the axial chemical potential can be made constant through the phase transition and is not affected by the condensation whatsoever.

- The unbroken $U(1)$ is a generic field and the two $U(1)$ 's are intertwined in a particular way by the anomaly. With this second interpretation, crossed anomalous correlators can be related to the response of the (broken) current to an external unscreened magnetic field, associated to the unbroken symmetry. This avoids any possible problem with the physical realization external magnetic fields contained in the bulk of the system.

Despite of the two possible interpretations, we will use a notation adapted to the first one. The action of the model contains a complex scalar field coupled to the vector sector

$$
\begin{aligned}
& \mathcal{L}=-\frac{1}{4} F_{M N} F^{M N}-\frac{1}{4} G_{M N} G^{M N}+\frac{\kappa}{2} \epsilon^{M A B C D} A_{M}\left(3 F_{A B} F_{C D}+G_{A B} G_{C D}\right) \\
& -\overline{D_{M} \Psi} D^{M} \Psi-m^{2} \bar{\Psi} \Psi .
\end{aligned}
$$

Here $F$ is the field strength for the vector gauge field $V$ and $G$ is the analogue for the axial gauge field $A$. Moreover $D_{M} \Psi=\partial_{M} \Psi-i V_{M} \Psi$. We consider AAA and AVV anomalies. The equations of motion for the background are the same as 12.1.19)-12.1.21), with an additional equation for the background axial gauge field $A(r)=K(r) d t$

$$
K^{\prime \prime}+\frac{3}{r} K^{\prime}=0
$$



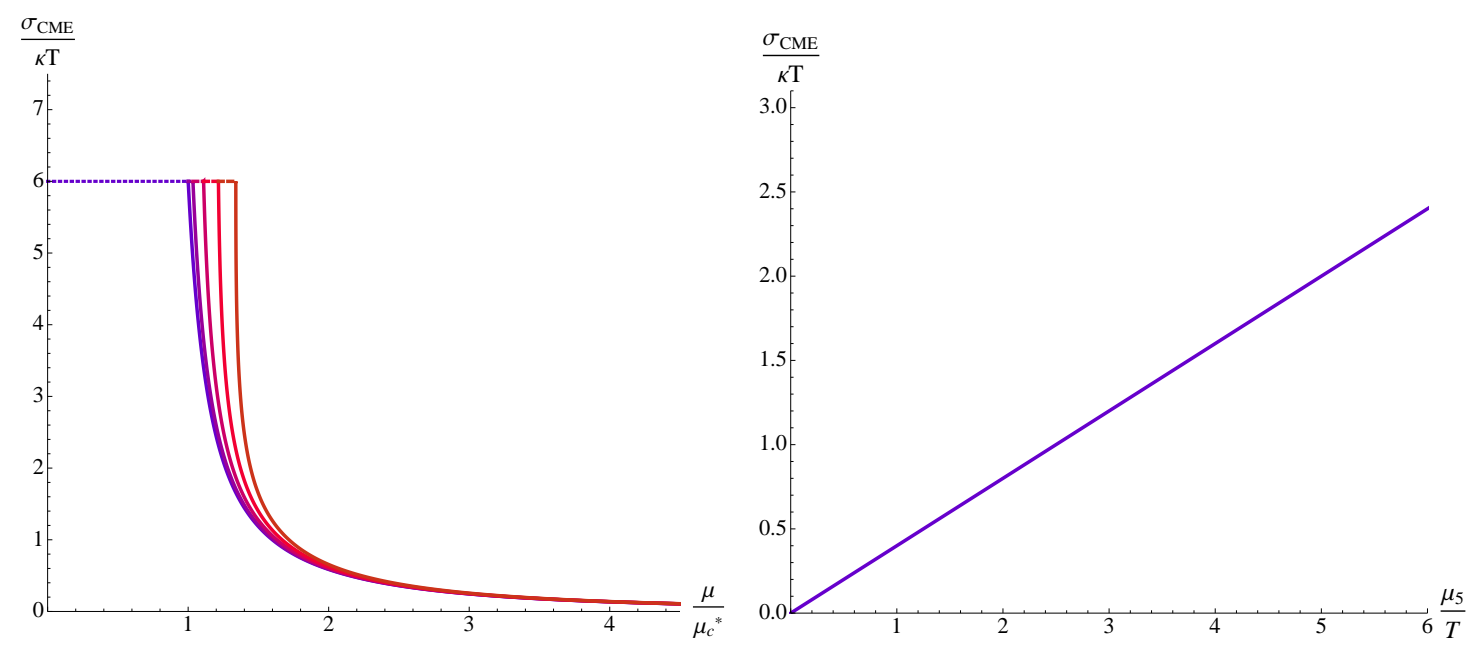

Figure 12.6: (Left) Chiral magnetic conductivity versus vector chemical potential with $\bar{\mu}_{5}=1$. Different lines correspond to different values of the superfluid velocity, with $\xi_{x} / T=0.1-2.1$. The best fit shows that for large enough values of $\bar{\mu}$ it decreases as $\sigma \sim 1 / \bar{\mu}^{2}$. (Right) This plot corresponds to $\sigma_{C M E} / T$ vs. axial chemical potential with $\mu / T=2.5$. The linear dependence with $\mu_{5}$, characteristic of the unbroken phase, remains unaltered. The conductivity depends linearly with $\kappa$.

which has a trivial analytic solution $K(r)=K_{0}-K_{1} / r^{2}$. The boundary conditions for the gauge fields are:

$$
\phi(r)_{r \rightarrow \infty} \sim \mu \quad V(r)_{r \rightarrow \infty} \sim \xi_{\{x, z\}} \quad K(r)_{r \rightarrow \infty} \sim \mu_{5}
$$

We impose again standard quantization for the scalar field. First we choose the supervelocity to point in the $x$-direction. On top of this we switch on the perturbations with non-vanishing frequency and momentum parallel to the supervelocity. The equations for the perturbations in the transverse sector can be found in Appendix 12.5.2. There is a wider set of correlators that we can study in this set up

$$
\begin{aligned}
\sigma_{55} & =\lim _{k \rightarrow 0} \frac{i}{2 k}\left\langle J_{A}^{y} J_{A}^{z}\right\rangle_{\mathcal{R}}(\omega=0, k) \\
\sigma_{C S E} & =\lim _{k \rightarrow 0} \frac{i}{2 k}\left\langle J_{V}^{y} J_{A}^{z}\right\rangle_{\mathcal{R}}(\omega=0, k) \\
\sigma_{C M E} & =\lim _{k \rightarrow 0} \frac{i}{2 k}\left\langle J_{V}^{y} J_{V}^{z}\right\rangle_{\mathcal{R}}(\omega=0, k)
\end{aligned}
$$

In the superfluid phase, after assuming that the supervelocity is transverse to the momentum, we can also consider the Kubo formulae related to the Chiral Electric Effect and the Chiral Charge Generation Effect

$$
\begin{array}{rlrl}
\sigma_{C C G E}^{A} & =\lim _{k \rightarrow 0} \frac{i}{2 k_{\perp}}\left\langle J_{A}^{0} J_{V}^{y}\right\rangle_{\mathcal{R}}(\omega=0, k) ; & \sigma_{C C G E}^{V}=\lim _{k \rightarrow 0} \frac{i}{2 k_{\perp}}\left\langle J_{V}^{0} J_{A}^{y}\right\rangle_{\mathcal{R}}(\omega=0, k) \\
\sigma_{C E E}^{A}=\lim _{k \rightarrow 0} \frac{i}{2 \omega}\left\langle J_{A}^{y} J_{V}^{z}\right\rangle_{\mathcal{R}}(\omega, k=0) ; & \sigma_{C E E}^{V}=\lim _{k \rightarrow 0} \frac{i}{2 \omega}\left\langle J_{V}^{y} J_{A}^{z}\right\rangle_{\mathcal{R}}(\omega, k=0)
\end{array}
$$

We expect them to receive different corrections due to the fact that the condensate distinguishes between the vector and the axial symmetry. Notice that our notation establishes 

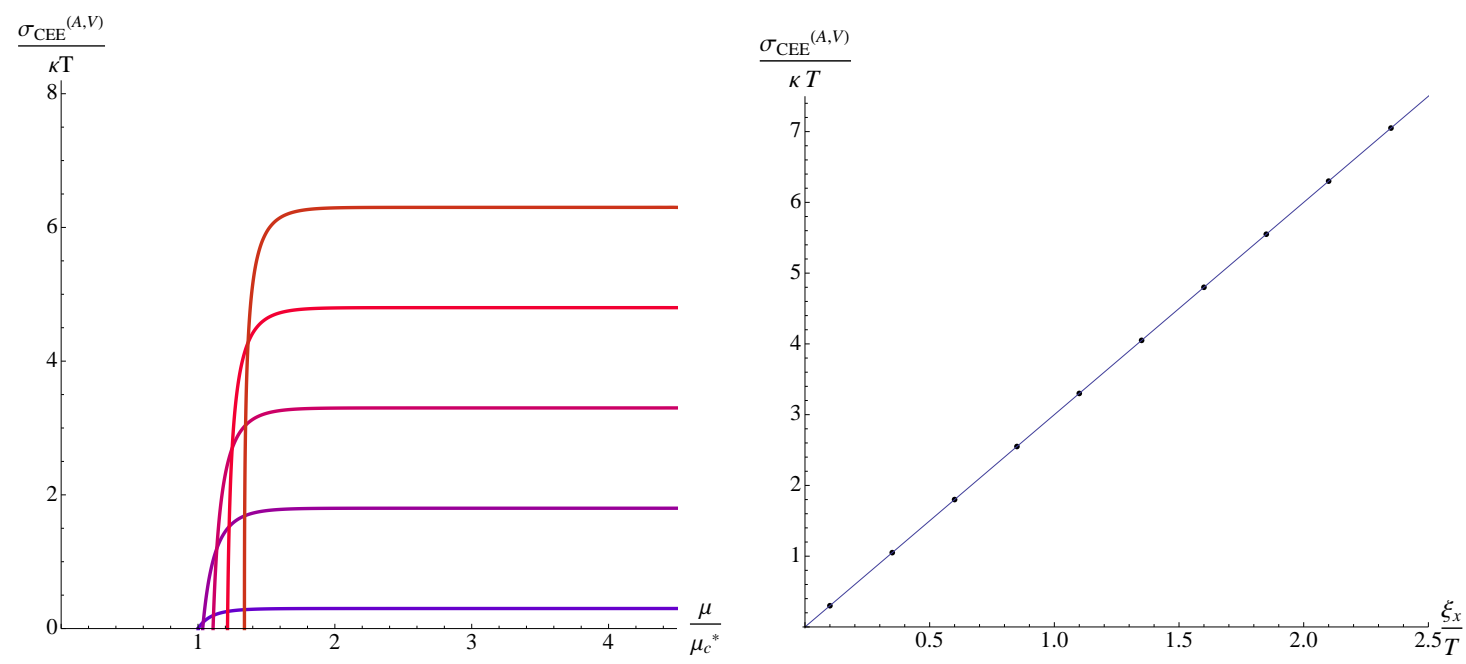

Figure 12.7: (Left) Chiral electric conductivities versus vector chemical potential at $\bar{\mu}_{5}=1$ and $\xi_{x} / T=0.1-2.1$ (bottom to top). Both $\sigma_{C E E}^{V} / T$ and $\sigma_{C E E}^{A} / T$ show the same behaviour . (Right) Chiral electric conductivities versus supervelocity at $\bar{\mu}_{5}=1$ in the region where they don't depend on $\mu$. The conductivity depends linearly with $\kappa$.

that, for example, $\rho_{A}=\sigma_{C C G E}^{A} B_{z}^{V}$ and $\rho_{V}=\sigma_{C C G E}^{V} B_{z}^{A}$.

Our results are as follows. On the one hand, the correlator $\left\langle J_{5}^{y} J_{5}^{z}\right\rangle$ does not get altered due to the condensate, and is linear in $\mu_{5}$, as depicted in Figure 12.4. The behavior could have been anticipated, since the on-shell action is diagonal in vector/axial sectors and it is clear that in the dynamical equations (12.5.78)-12.5.79) the mixing between $a_{y}$ and $a_{z}$ is independent of the condensate.

On the other hand, the results concerning $\sigma_{C S E}$ are summarized in Figure 12.5. This conductivity acts similarly to that encountered in the first section. This was expected by the form of the equations of motion: in this model, the correlator mixing between $a_{y}$ and $v_{z}$ is mediated by the same background fields as in the model with only axial symmetry. Remarkably, unlike the case with a $U(1)^{3}$ anomaly, at large values of $\bar{\mu}$ we obtain 7

$$
\frac{\sigma_{C S E}\left(\frac{\bar{\mu}}{\bar{\mu}_{c}}>>1\right)}{\kappa \mu}=2.998 \approx \frac{\sigma_{C S E}\left(\bar{\mu}_{c}\right)}{2 \kappa \mu},
$$

independently of the superfluid velocity. This result indicates that the $T \rightarrow 0$ behaviour is strongly dependent on the structure of the broken symmetries and the interplay of the anomalies. Moreover, the conductivity does not depend on the axial chemical potential (right plot).

Finally, let us comment on the $\sigma_{C M E}$. The results are displayed in Figure 12.6 . We find a linear dependence on $\bar{\mu}_{5}$, as expected. However, in the presence of the condensate we observe a new dependence on the vector chemical potential, which is absent in the unbroken phase. The chemical potential diminishes the value of the CMC strongly and it

\footnotetext{
${ }^{7}$ The numerical value $\sigma_{C S E}\left(T_{c}\right) /(\kappa \mu) \approx 6$ depends on the strength of the $\kappa$-term in the equations of motion and is not of fundamental importance, for it can be easily rescaled (compare to Section 12.1).
} 
tends to zero for large values of $\bar{\mu}$ as

$$
\frac{\sigma_{C M E}\left(\frac{\bar{\mu}}{\bar{\mu}_{c}}>>1\right)}{\kappa T} \approx g \frac{1}{\bar{\mu}^{2}}
$$

with a numerical value for $g \approx 2.15$. We elaborate on this in Section 12.3 .

For the chiral transport coefficients associated to the CEE, we observe that correlators of the form $\left\langle J_{A} J_{A}\right\rangle(k=0)$ and $\left\langle J_{V} J_{V}\right\rangle(k=0)$ vanish identically. Concerning the ones mixing axial and vector currents, we observe that $\sigma_{C E E}^{V}=\sigma_{C E E}^{A} \equiv \sigma_{C E E}^{(V, A)}$. The result is depicted in Figure 12.7. Fitting the right plot to a parabola, we get

$$
\frac{\sigma_{C E E}^{(V, A)}\left(\frac{\bar{\mu}}{\bar{\mu}_{c}}>>1\right)}{\kappa T}=3.003 \frac{\xi_{x}}{T} .
$$

with remarkable precision.

\subsection{1 $U(1) \times U(1)$ model with transverse supervelocity}

As we did in the previous model, in order to study the CCGE we switch on perturbations with non-zero frequency and momentum pointing in the $x$-direction, transverse to the superfluid velocity ( $z$-direction). The system of equations with transverse supervelocity can be found in Appendix 12.5.3. We report the results on the CCGC in Figure 12.8. As shown in there are now two different conductivities related to the CCGE, which we denote $\sigma_{C C G E}^{(V)}$ and $\sigma_{C C G E}^{(A)}$ They exhibit a very different behavior close to $\bar{\mu}_{c}$; the conductivity $\sigma_{C C G E}^{(V)}$ is similar to the one found in Section 12.1.2, whereas $\sigma_{C C G E}^{(A)}$ looks like the CEC, with a good continuous behavior at the phase transition. We comment on those differences in Section 12.3. At low temperatures, however, both $\sigma_{C C G E}^{(A)}$ and $\sigma_{C C G E}^{(V)}$ tend to the same value and the dependence with the supervelocity is linear (Figure 12.9). A quadratic fit yields

$$
\frac{\sigma_{C C G E}^{(V, A)}\left(\frac{\bar{\mu}}{\bar{\mu}_{c}}>>1\right)}{\kappa T}=3.003 \frac{\xi_{z}}{T} .
$$

Remarkably enough, we point out that the conductivity

$$
\sigma_{C C G E(V V)}=\lim _{k \rightarrow 0} \frac{i}{2 k_{\perp}}\left\langle J_{V}^{0} J_{V}^{y}\right\rangle_{\mathcal{R}}(\omega=0, k),
$$

depicted in Figure 12.10, is not negligible. In principle we could have anticipated it to vanish because of the structure of the anomalies included in the Lagrangian (12.2.32). As shown in the plot, this only occurs far enough form the phase transition. This effect points towards an "effective VVV anomaly" (see also the results concerning the CMC) that is present close to the phase transition.

\subsection{Conclusions, Educated guesses and Future directions}

We have analyzed the explicit form of the chiral conductivities in two holographic models with $U(1)$ and $U(1) \times U(1)$ symmetries that develop a scalar condensate at finite superfluid velocity. We have presented an explicit calculation of CEE by using a suitable Kubo formula, which allowed us to prove in a robust way that the CEC is in general not 

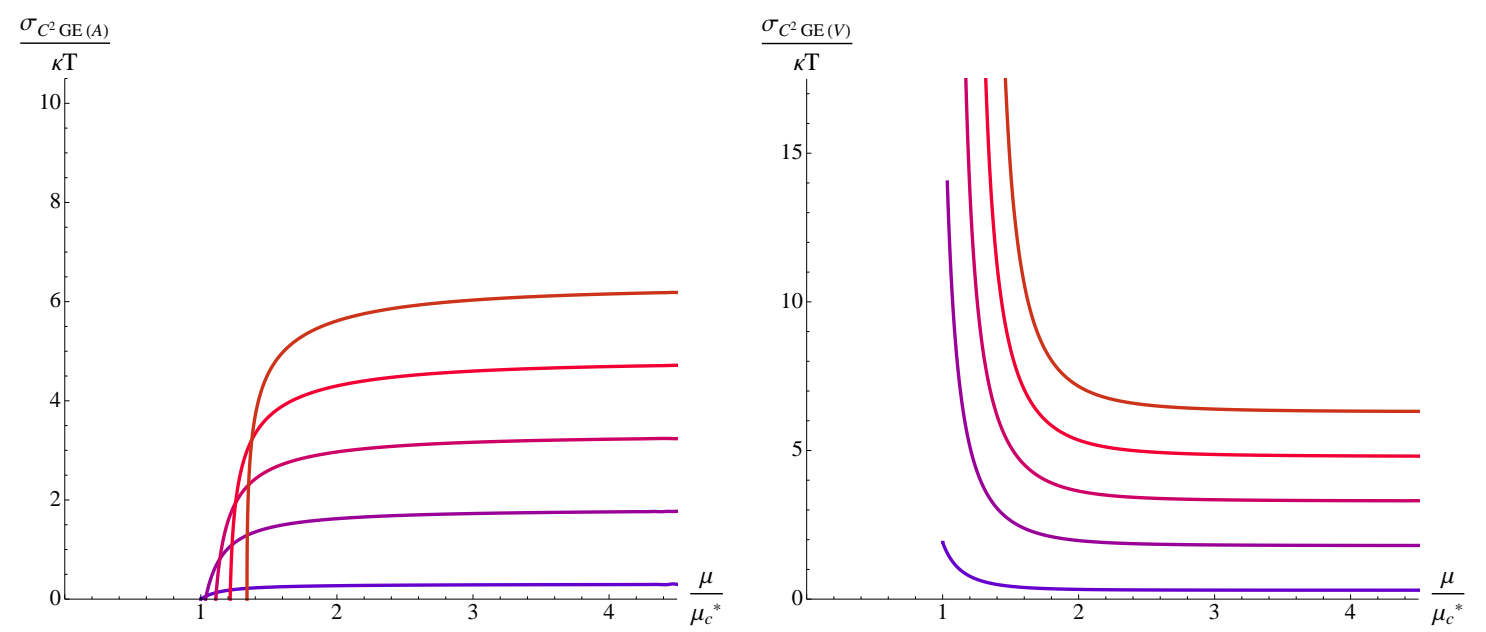

Figure 12.8: $\quad \sigma_{C^{2} G E}^{(A)} / T$ (Left) and $\sigma_{C^{2} G E}^{(V)} / T$ (Right) versus vector chemical potential at $\bar{\mu}_{5}=1$ and $\xi_{x} / T=0.1-2.1$ (bottom to top). For large enough values of the chemical potential both conductivities show the same behaviour. Both depend linearly with $\kappa$.

vanishing in superfluids.

Moreover, by means of the Kubo formulae we have found an effect whose existence, as far as we are aware, had not been emphasized before in the literature. This induces the "generation" of axial charge in the presence of supervelocity and a magnetic field

$$
\rho_{A} \propto \vec{\xi} \cdot \vec{B}
$$

Such a term has interesting consequences. For instance, the Chiral Magnetic Effect would be dynamically produced in a superfluid in the presence of an external magnetic field aligned with the supervelocity. We believe this term deserves more investigation in the future, in order to fully understand the mechanism by which charge is "generated", as well as to analyze the implications that it could lead to.

In addition, we have found generic corrections, due to the background condensate, to all of the anomalous conductivities. Such corrections seem to take a constant value as $T \rightarrow 0$ in all of the cases. We observe that such value is model-dependent, but seems to be strongly constrained by the number of broken symmetries and the interplay between the anomalies.

Section 12.1 is devoted to the study of the chiral transport of a broken anomalous $U(1)$ symmetry. At $\xi=0$, we found the result previously pointed out, namely, the value of the conductivity is $1 / 3$ of that in the unbroken phase, i.e,

$$
\sigma_{55}(T \rightarrow 0) \approx \frac{8 \kappa}{3} \mu_{5}=\frac{\sigma_{55}\left(T_{c}\right)}{3} .
$$

This fact turns out not to be affected when a supervelocity parallel to the momentum is considered. Moreover, as soon as supervelocity is considered, we have two new anomalous effects present: The Chiral Electric Effect and the Chiral Charge Generation Effect. We proposed suitable Kubo formulae for both the CEE and CCGE and computed their value, finding that both become independent from the chemical potential at sufficiently low temperatures. Moreover, their dependence with the superfluid velocity is linear, i.e.,

$$
\sigma_{C E E}(T \rightarrow 0) \approx \sigma_{C C G E}(T \rightarrow 0) \approx \frac{8 \kappa}{3} \xi_{x}
$$




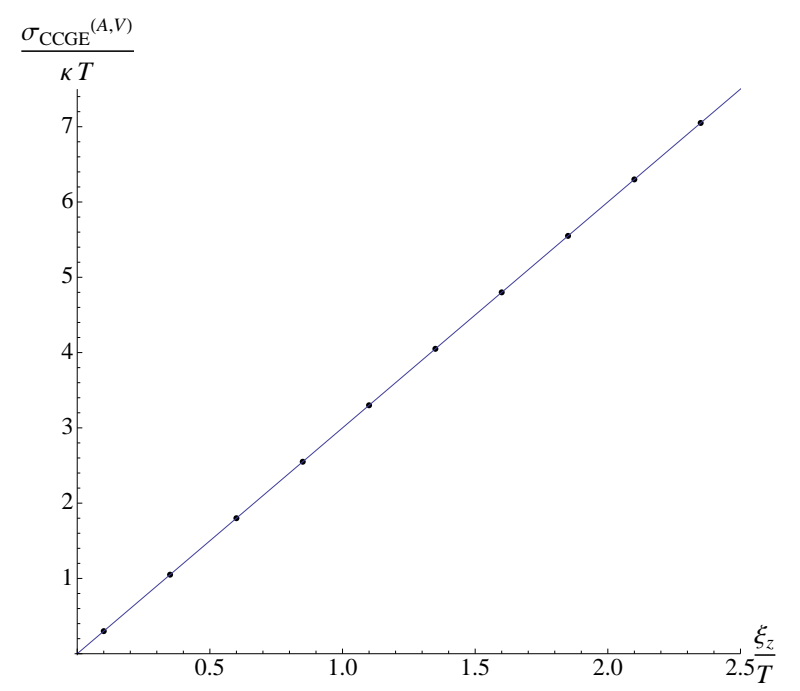

Figure 12.9: Both conductivities $\sigma_{C^{2} G E}^{(A, V)}$ show the same dependence on the vector chemical potential $\mu$ and the supervelocity $\xi_{z}$ for large enough values of $\mu$. The slope coincides with the slope for the CEC, despite the radically different behaviour close to the phase transition.

Section 12.2 deals with two $U(1)$ global symmetries, giving rise to a more rich set of anomalous conductivities with different behaviors once one of the $U(1)$ symmetries gets spontaneously broken. The transport coefficient $\sigma_{55}$ remains the same as in the unbroken phase, but the CMC now acquires a dependence of the vector chemical potential that makes it vanish as we lower the temperature. That result suggests that the charged particles stored in the condensate (forming "cooper pairs") do not contribute to the CMC, which hence vanishes at sufficiently low temperatures. The decrease of the CMC seems to be following a law of the form $\sigma_{C M E} / T \sim g / \bar{\mu}^{2}$, with $g \approx 2.15$. The scaling of $\sigma_{C M E}$ with the axial chemical potential is the usual one, namely $\sigma_{C M E} \sim \mu_{A}$. Finally, the CSE decreases up to $1 / 2$ the value that it presents in the unbroken phase, yielding

$$
\sigma_{C S E}(T \rightarrow 0) \approx \frac{6 \kappa}{2} \mu=\frac{\sigma_{C S E}\left(T_{c}\right)}{2} .
$$

These results do not get altered when inducing supervelocity. Furthermore, we observe $\sigma_{C E E}^{V}=\sigma_{C E E}^{A}$, both presenting a qualitative behavior that is similar to the one of Section 12.1 however the scaling with supervelocity is now

$$
\sigma_{C E E}^{(V, A)}(T \rightarrow 0) \approx 3 \kappa \xi_{x}
$$

Finally, $\sigma_{C C G E}^{V} \neq \sigma_{C C G E}^{A}$ close to the phase transition. At low temperatures both tend to the same value and the same dependence on supervelocity, namely

$$
\sigma_{C^{2} G E}^{(V, A)}(T \rightarrow 0) \approx 3 \kappa \xi_{z}
$$

\subsubsection{On the Low temperature behaviour of the Chiral Conductivities}

A simple argument by which the CCGE is expected to arise in superfluids is the following. Imagine that we have free Chiral fermions coupled to an electromagnetic field $A_{\mu}$.

$$
\mathcal{L}=\bar{\psi}\left(V_{\mu}-A_{\mu}\right) \gamma^{\mu} \psi
$$




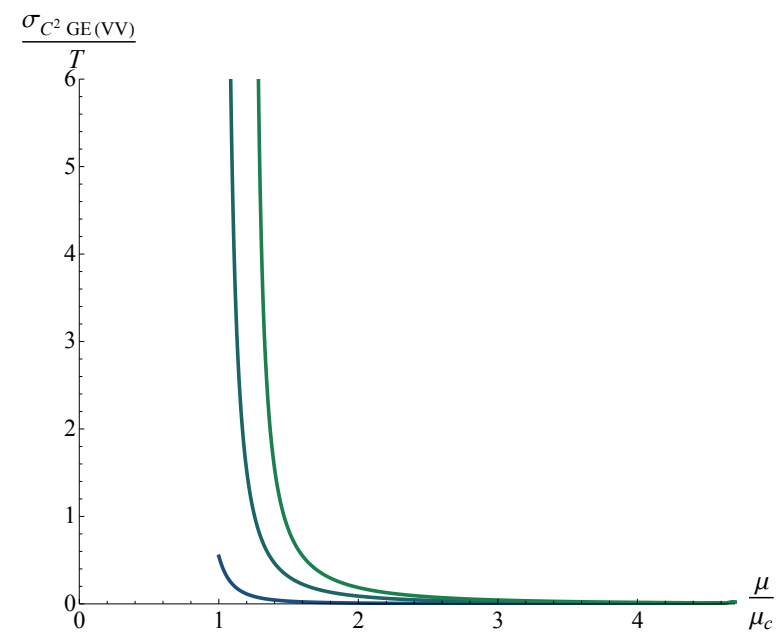

Figure 12.10: Plot of the $\sigma_{C^{2} G E(V V)}$ conductivity (defined in the text) versus vector chemical potential for several values of the supervelocity.

We also couple them to an external field $V_{\mu}$ associated to a $U(1)$ symmetry that gets spontaneously broken. The axial current $j_{\text {axial }}^{\mu}=\bar{\psi} \gamma^{\mu} \gamma^{5} \psi$ is anomalous. Hence, in general

$$
\partial_{\mu} j_{\text {axial }}^{\mu}=a F \wedge F+b G \wedge F+c G \wedge G
$$

where $a, b$ and $c$ are coefficients; $F$ and $G$ are the stress-tensors associated to $A_{\mu}$ and $V_{\mu}$ respectively. There is no external axial field. Let us concentrate on the term proportional to $b$; due to the Bianchi identities, it can be rewritten as $b \partial_{\mu}\left(\epsilon^{\mu \nu \rho \lambda} V_{\nu} F_{\rho \lambda}\right){ }^{8}$. At this point, we substitute the actual value of $V_{\mu}$, which, assuming that $\mu=0$, corresponds to $V_{\mu}=\left(0, \xi_{x}, 0,0\right)$, Assuming that $j_{\text {axial }}^{\mu}$ does not depend on the position, we find, in momentum space

$$
\omega j_{\text {axial }}^{\mu}=\omega b \epsilon^{\mu x \rho \lambda} \xi_{x} F_{\rho \lambda}+\ldots
$$

leading to both the Chiral Electric Effect and the Chiral Charge Generation Effect, i.e,

$$
\begin{aligned}
& j_{\text {axial }}^{y} \sim b \epsilon^{y x t z} \xi_{x} E_{z} \\
& j_{\text {axial }}^{t} \sim b \epsilon^{t x y z} \xi_{x} B_{x}
\end{aligned}
$$

The above argument "with the hands" leads us to some notion of covariantization of those effect: 10 This would imply that for the $U(1)^{3}$ anomaly, the anomalous contribution to the current can be recast in a covariant form

$$
J_{\text {anom }}^{\mu}(T \rightarrow 0)=\Sigma_{S C E}^{\mathcal{A}} \epsilon^{\mu \nu \rho \lambda} u_{\nu}^{S} F_{\rho \lambda}+\ldots
$$

\footnotetext{
${ }^{8}$ Notice that, since the symmetry is spontaneously broken, in principle we have to substitute $V_{\mu} \rightarrow$ $V_{\mu}-\partial_{\mu} \phi$, where $\phi$ is the Goldstone mode. However, for simplicity we stick to a gauge in which $\phi=0$. This will not influence our conclusions.

${ }^{9}$ One can consider $\xi \rightarrow \xi e^{-i \omega t}$ instead, to bring down the frequency in 12.3 .53 consistently. At the end of the calculation all the $\omega$ factors will cancel.

${ }^{10} \mathrm{~A}$ cautionary remark is in order here. It is not clear to us whether an argument such as the one presented here gives the complete answer, i.e. whether one can associate the parameter $b$ in 12.3 .55 to the actual $\sigma_{C C G E}$. Most likely one cannot. The reason for our concerns is that, for instance, the reasoning does not distinguish between covariant/consistent currents and overlooks the subtleties associated to the introduction of chemical potential/supervelocity in the presence of anomalous symmetries. However, we believe that it serves to ilustrate the kind of transport phenomena that we expect, for it works at the formal level.
} 
where $u^{S \mu}=-\mu u^{\mu}+\zeta_{\nu} P^{\nu \mu}$ is the (non-normalized) superfluid velocity and the "..." indicate possible corrections due to vorticity. This covariant form of the response can be analyzed numerically by establishing the numerical universality (up to the form of the interplay between the anomaly $\mathcal{A}$ and the broken symmetries) of the coefficient $\Sigma_{S C E}^{\mathcal{A}}$. Our results suggest that the superfluid component (the only one present at zero temperature) gives a contribution of the form 12.3.56 with

$$
\Sigma_{S C E}^{A A A}=\frac{C}{3}
$$

being $C$ a number that is fully determined by the anomaly coefficient.

For the $U(1) \times U(1)$ model the at zero temperatures there exists a subset of non-vanishing chiral conductivities for which (12.3.56) applies, with

$$
\Sigma_{S C E}^{A V V}=\frac{C}{2} .
$$

Equations 12.3.57) and (12.3.58) are very suggestive. The nature of the number in the denominator of $\Sigma_{S C E}^{A}$ appears to be determined by the spontaneously broken symmetries that are contained in the anomaly responsible for the chiral conductivity under consideration.

Furthermore, one could ask whether the conclusions presented here are universal, i.e. valid for all holographic s-wave superfluids or even beyond the holographic approach. If 12.3.57) and 12.3.58 held in general, it would imply that at zero temperature the anomalous conductivities have a robust value, entirely determined by anomaly coefficients plus the interplay between the broken symmetries and the anomalies.

We would also like to emphasize that formula 12.0 .15 allows us to extract the coefficient termed $g_{1}\left(T, \mu / T, \xi^{2} / T^{2}\right)$ in [96]. At low temperatures, our numerical results for the CCGC and $\sigma_{55}$ for the $U(1)^{3}$ anomaly are perfectly compatible with

$$
g_{1}(\bar{\mu}>>1)=-\frac{C}{3} \frac{\mu}{T}
$$

In the case of the AVV anomaly, the compatibility seems to be not that straightforward. In the notation of $203, \sigma_{55} \sim\left(2 T g_{1}+\mu C\right)$. The coefficient $g_{1}$ is continuous at the phase transition but its derivative is not (see Figure 12.1) and hence $\sigma_{C C G E} \sim g_{1, \nu}$ is not continuous at $\bar{\mu}_{c}$. This fact explains why we do not observe that the CCGC vanishes at the phase transition.

Finally, let us mention that the electric field $E_{x}=\partial_{[t} A_{x]}$ is a gauge invariant source in our setup, so assuming that $j^{y} \sim \sigma_{C E E} E_{x}$ only, we would have expected

$$
\left.\frac{i}{\xi_{z}} \lim _{\omega \rightarrow 0} \partial_{\omega} \mathcal{G}_{r a}^{y x}(\omega, k)\right|_{k_{y}=k_{x}=0}=\left.\frac{i}{\xi_{z}} \lim _{k_{x} \rightarrow 0} \partial_{k_{x}} \mathcal{G}_{r a}^{y t}(\omega, k)\right|_{k_{y}=k_{x}=0}
$$

to hold by gauge invariance. Here $\mathcal{G}_{r a}^{\mu \nu}$ are retarded correlators and the subindex "ra" represents the correct combination of time and anti-time ordered sources with respect to which we vary the generating functional.

Notice that the right hand side of equation 12.3 .60 is also the Kubo formula for $\sigma_{C C G E}$, and therefore $\sigma_{C C G E}=\sigma_{C E E}$ should be enforced by gauge invariance of the external sources. This is not what we observe, compare Figures 12.2 and 12.3 . The reason is that the constitutive relation of the current receives contributions from terms other than the one associated to the $\mathrm{CEE}$ and therefore the limits taken in 12.3 .60 capture the influence of gauge-invariant sources that are not the electric field. Remarkably, the effect of those 

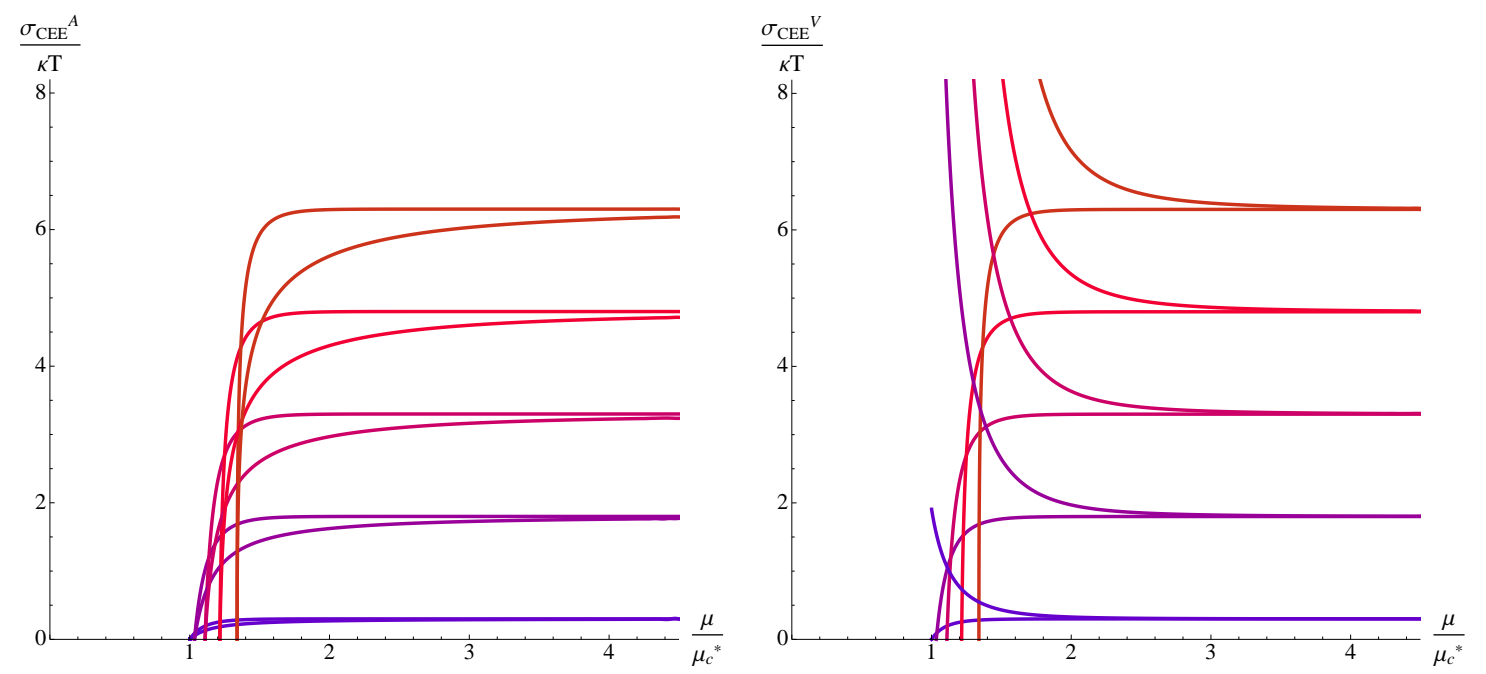

Figure 12.11: $\quad \sigma_{C E E}^{(A)} / T$ (Left) and $\sigma_{C E E}^{(V)} / T$ (Right) versus vector chemical potential at $\bar{\mu}_{5}=1$ and $\xi_{x} / T=0.1-2.1$ (bottom to top) computed in the two different kinematic limits allowed by gauge invariance. For large enough values of the chemical potential the lines overlap. Notice that one of the limits corresponds to the CCGC.

other sources seems to vanish at low temperatures, as shown in Figure 12.11 , for, at $T \rightarrow 0$, we recover (12.3.60). This supports the validity of the relation 12.3.56).

For future analysis, a possible direction concerns he computation of the Chiral Vortical Conductivity. This amounts to studying the system with backreaction. However, the complicated form of the holographic gauge-gravitational anomaly introduces important difficulties. Moreover, it would also be interesting to analyze the case in which the pattern of broken symmetries is $U(2) \rightarrow U(1)$, for in that case it is known that the spectrum of low-energy excitations is qualitatively different and this could affect the anomalous conductivities. 


\section{Appendix of Part IV}

\subsection{Computing the Conductivities}

To compute the conductivities we have followed the method developed in [51].

We rearrange the perturbations in a vector $\Phi\left(r, x^{\mu}\right)$ and work with the Fourier transformed quantity

$$
\Phi\left(r, x^{\mu}\right)=\int \frac{d^{d} k}{(2 \pi)^{d}} \Phi_{k}^{I}(r) e^{-i \omega t+i \vec{k} \vec{x}}
$$

with $\Phi_{k}(r)$ being

$$
\Phi_{k}^{\top}(u)=\left(A_{t}(r), A_{x}(r), A_{z}(r), \ldots\right)
$$

(the specific structure depends on the case at hand, the number of coupled fields, etc.). The general form of the boundary action is [51]

$$
\delta S^{(2)}=\int \frac{d^{d} k}{(2 \pi)^{d}}\left[\Phi_{-k}^{I} \mathcal{A}_{I J} \Phi_{k}^{\prime J}+\Phi_{-k}^{I} \mathcal{B}_{I J} \Phi_{k}^{J}\right]
$$

where the prime stands for $d / d r$. To calculate the retarded correlators we solve the equations for the perturbations with infalling boundary conditions, on the one hand, and boundary conditions $\Phi_{k}^{I}(r \rightarrow \infty)=\phi_{k}^{I}$ on the other. This procedure should give us the desired Green's functions, after taking the variation of 12.4.63 with respect to the fields at large values of $r$. Moreover, if

$$
\Phi_{k}^{I}(r)=F_{J}^{I}(k, r) \phi_{k}^{J}
$$

then $F_{J}^{I}(k, r \rightarrow \infty)=1$ is the bulk-to-boundary propagator. The retarded two-point functions, from which we are able to read directly the transport coefficients, are then computed as

$$
G_{I J}^{\mathcal{R}}(k, r \rightarrow \infty)=-2 \lim _{r \rightarrow \infty}\left(\mathcal{A}_{I M}\left(F_{J}^{M}(k, r)\right)^{\prime}+\mathcal{B}_{I J}\right)
$$

The $\mathcal{A}_{I J}$ and $\mathcal{B}_{I J}$ matrices depend only on the background and also upon the model under consideration. We provide their values below

\subsection{1 $U(1)$ model: $\mathcal{A}_{I J}$ and $\mathcal{B}_{I J}$ matrices}

The matrices turn out to be independent of the supervelocity and its direction, once we neglect the contribution of the Chern-Simons term to define the covariant currents. 
We get

$$
\begin{array}{r}
\mathcal{A}=-\frac{1}{2} r f(r) \operatorname{Diag}(1,1) \\
\mathcal{B}=0 \\
\mathcal{B}_{\mathrm{CT}}=\frac{\ln r}{4}\left(\frac{k^{2} \sqrt{f(r)}}{r}-\frac{\omega^{2} r}{\sqrt{f(r)}}\right) \operatorname{Diag}(1,1)
\end{array}
$$

Notice that the counterterms do not contribute to the anomalous transport coefficients, for $\mathcal{B}_{\mathrm{CT}}$ only has diagonal entries, which furthermore are of second order in $\omega$ and $k$.

\subsection{2 $U(1) \times U(1)$ model: $\mathcal{A}_{I J}$ and $\mathcal{B}_{I J}$ matrices}

In this case we get the same results as before, independently for the axial and vector fields, namely

$$
\begin{array}{r}
\mathcal{A}_{\text {axial }}=-\frac{1}{2} r f(r) \operatorname{Diag}(1,1) \\
\mathcal{B}_{\text {axial }}=0 \\
\mathcal{B}_{\mathrm{CT}}^{\text {axial }}=\frac{\ln r}{4}\left(\frac{k^{2} \sqrt{f(r)}}{r}-\frac{\omega^{2} r}{\sqrt{f(r)}}\right) \operatorname{Diag}(1,1)
\end{array}
$$

and

$$
\begin{array}{r}
\mathcal{A}_{\text {vector }}=-\frac{1}{2} r f(r) \operatorname{Diag}(1,1) \\
\mathcal{B}_{\text {vector }}=0 \\
\mathcal{B}_{\mathrm{CT}}^{\text {vector }}=\frac{\ln r}{4}\left(\frac{k^{2} \sqrt{f(r)}}{r}-\frac{\omega^{2} r}{\sqrt{f(r)}}\right) \operatorname{Diag}(1,1)
\end{array}
$$




\subsection{Equations of Motion}

\subsubsection{Momentum transverse to the supervelocity for the $U(1)$ model}

$$
\begin{array}{r}
0=f \rho^{\prime \prime}+\left(f^{\prime}+\frac{3 f}{r}\right) \rho^{\prime}+\left(\frac{\omega^{2}}{f}+\frac{\phi^{2}}{f}-\frac{V^{2}}{r^{2}}-\frac{k^{2}}{r^{2}}-m^{2}\right) \rho+\frac{2 i \omega \phi}{f} \delta+2 a_{t} \Psi \frac{\phi}{f}-2 \frac{a_{z}}{r^{2}} \Psi V \\
(12.5 .69) \\
0=f \delta^{\prime \prime}+\left(f^{\prime}+\frac{3 f}{r}\right) \delta^{\prime}+\left(\frac{\omega^{2}}{f}+\frac{\phi^{2}}{f}-\frac{V^{2}}{r^{2}}-\frac{k^{2}}{r^{2}}-m^{2}\right) \delta-\frac{2 i \omega \phi}{f} \rho-i \Psi \omega \frac{a_{t}}{f}-k \frac{i}{r^{2}} \Psi a_{x} \\
(12.5 .70) \\
0=f a_{t}^{\prime \prime}+\frac{3 f}{r} a_{t}^{\prime}-\left(\frac{k^{2}}{r^{2}}+2 \Psi^{2}\right) a_{t}-\frac{\omega k}{r^{2}} a_{x}-4 \Psi \phi \rho-2 i \omega \Psi \delta-16 i k \kappa \frac{f}{r^{3}} V^{\prime} a_{y} \\
(12.5 .71) \\
0=f a_{x}^{\prime \prime}+\left(f^{\prime}+\frac{f}{r}\right) a_{x}^{\prime}+\left(\frac{\omega^{2}}{f}-2 \Psi^{2}\right) a_{x}+\frac{\omega k}{f} a_{t}+2 i k \Psi \delta+\frac{16 i \kappa}{r} \omega V^{\prime} a_{y} \\
(12.5 .72) \\
f a_{y}^{\prime \prime}+\left(f^{\prime}+\frac{f}{r}\right) a_{y}^{\prime}+\left(\frac{\omega^{2}}{f}-\frac{k^{2}}{r^{2}}-2 \psi^{2}\right) a_{y}+16 i k \frac{\kappa}{r} \phi^{\prime} a_{z}-\frac{16 i \kappa}{r} V^{\prime}\left(\omega a_{x}+k a_{t}\right)=0 \\
(12.5 .73) \\
\left(f^{\prime}+\frac{f}{r}\right) a_{z}^{\prime}+\left(\frac{\omega^{2}}{f}-\frac{k^{2}}{r^{2}}-2 \psi^{2}\right) a_{z}-16 i k \frac{\kappa}{r} \phi^{\prime} a_{y}-4 V \Psi \rho=0
\end{array}
$$

and the constraint

$$
0=\frac{i \omega}{f} a_{t}^{\prime}+\frac{i k}{r^{2}} a_{x}^{\prime}+2 \Psi^{\prime} \delta-2 \Psi \delta^{\prime}
$$

Where $a_{i}$ are the perturbations of the axial gauge field. $\rho$ and $\delta$ are the real and imaginary parts of the perturbations of the scalar field, respectively. Momentum points in the $x$-direction, transverse to the superfluid velocity that points in the $z$-direction. We observe that now the equations become more complicated, with the perturbations of the scalar coupled to all the fields, including the transverse sector. This can imply that the Quasinormal Modes now get affected by the anomaly. 


\subsubsection{Momentum parallel to the supervelocity for the $U(1)$ model}

The equations for the relevant sector with momentum aligned to the supervelocity read

$$
\begin{array}{r}
v_{y}^{\prime \prime}+\left(\frac{f^{\prime}}{f}+\frac{1}{r}\right) v_{y}^{\prime}+\frac{1}{f}\left(\frac{\omega^{2}}{f}-\frac{k^{2} L^{2}}{r^{2}}-2 \psi^{2}\right) v_{y}+12 i k \frac{\kappa L}{r f} \phi^{\prime} a_{z}+12 i k \frac{\kappa L}{r f} K^{\prime} v_{z} \\
+12 i \omega \frac{\kappa L}{r f} V^{\prime} a_{z}=0 \\
(12.5 .76) \\
v_{z}^{\prime \prime}+\left(\frac{f^{\prime}}{f}+\frac{1}{r}\right) v_{z}^{\prime}+\frac{1}{f}\left(\frac{\omega^{2}}{f}-\frac{k^{2} L^{2}}{r^{2}}-2 \psi^{2}\right) v_{z}-12 i k \frac{\kappa L}{r f} \phi^{\prime} a_{y}-12 i k \frac{\kappa L}{r f} K^{\prime} v_{y} \\
-12 i \omega \frac{\kappa L}{r f} V^{\prime} a_{y}=0
\end{array}
$$

$a_{y}^{\prime \prime}+\left(\frac{f^{\prime}}{f}+\frac{1}{r}\right) a_{y}^{\prime}+\frac{1}{f}\left(\frac{\omega^{2}}{f}-\frac{k^{2} L^{2}}{r^{2}}\right) a_{y}+12 i k \frac{\kappa L}{r f} \phi^{\prime} v_{z}+12 i k \frac{\kappa L}{r f} K^{\prime} a_{z}+12 i \omega \frac{\kappa L}{r f} V^{\prime} v_{z}=0$

$a_{z}^{\prime \prime}+\left(\frac{f^{\prime}}{f}+\frac{1}{r}\right) a_{z}^{\prime}+\frac{1}{f}\left(\frac{\omega^{2}}{f}-\frac{k^{2} L^{2}}{r^{2}}\right) a_{z}-12 i k \frac{\kappa L}{r f} \phi^{\prime} v_{y}-12 i k \frac{\kappa L}{r f} K^{\prime} a_{y}-12 i \omega \frac{\kappa L}{r f} V^{\prime} v_{y}=0$

where $v_{\{y, z\}}$ and $a_{\{y, z\}}$ are the vector and axial perturbations respectively. Momentum points in the $x$-direction, parallel to the supervelocity. Note that only the vector component couples to the condensate, as could have been anticipated. This equations decouple from the equations for the rest of perturbations. 
12.5.3 Momentum transverse to the supervelocity for the $U(1) \times U(1)$ model

The equations read

$$
\begin{aligned}
& f \rho^{\prime \prime}+\left(f^{\prime}+\frac{3 f}{r}\right) \rho+\left(\frac{\omega^{2}+\phi^{2}}{f}-\frac{k^{2}+V^{2}}{r^{2}}-m^{2}\right) \rho-\frac{2}{r^{2}} \psi V v_{z}+\frac{2 \phi}{f}\left(\psi v_{t}+i \omega \delta\right)=0 \\
& f \delta^{\prime \prime}+\left(f^{\prime}+\frac{3 f}{r}\right) \delta+\left(\frac{\omega^{2}+\phi^{2}}{f}-\frac{k^{2}+V^{2}}{r^{2}}-m^{2}\right) \delta-\frac{i}{r^{2}} \psi k v_{x}-\frac{i \omega}{f}\left(\psi v_{t}+2 \phi \rho\right)=0 \\
& f v_{t}^{\prime \prime}+\frac{3 f}{r} v_{t}^{\prime}-\left(\frac{k^{2}}{r^{2}}+2 \psi^{2}\right) v_{t}-\frac{\omega k}{r^{2}} v_{x}-2 i \omega \psi \delta-4 \phi \psi \rho-12 i k \frac{\kappa f}{r^{3}} V^{\prime} a_{y}=0 \\
& f v_{x}^{\prime \prime}+\left(f^{\prime}+\frac{f}{r}\right) v_{x}^{\prime}+\left(\frac{\omega^{2}}{f}-2 \psi^{2}\right) v_{x}+\frac{\omega k}{f} v_{t}+2 i k \psi \delta+12 i \omega \frac{\kappa}{r} V^{\prime} a_{y}=0 \\
& f v_{y}^{\prime \prime}+\left(f^{\prime}+\frac{f}{r}\right) v_{y}^{\prime}+\left(\frac{\omega^{2}}{f}-\frac{k^{2}}{r^{2}}-2 \psi^{2}\right) v_{y}+12 i k \frac{\kappa}{r} \phi^{\prime} a_{z}+12 i k \frac{\kappa}{r} K^{\prime} v_{z}- \\
& 12 i \omega \frac{\kappa}{r} V^{\prime} a_{x}-24 i k \frac{\kappa}{r} V^{\prime} a_{t}=0 \\
& f v_{z}^{\prime \prime}+\left(f^{\prime}+\frac{f}{r}\right) v_{z}^{\prime}+\left(\frac{\omega^{2}}{f}-\frac{k^{2}}{r^{2}}-2 \psi^{2}\right) v_{z}-4 V \psi \rho-12 i k \frac{\kappa}{r} \phi^{\prime} a_{y}-12 i k \frac{\kappa}{r} K^{\prime} v_{y}=0 \\
& f a_{t}^{\prime \prime}+\frac{3 f}{r} a_{t}^{\prime}-\frac{k^{2}}{r^{2}} a_{t}-\frac{\omega k}{r^{2}} a_{x}-12 i k \frac{\kappa f}{r^{3}} V^{\prime} v_{y}=0 \\
& f a_{x}^{\prime \prime}+\left(f^{\prime}+\frac{f}{r}\right) a_{x}^{\prime}+\frac{\omega^{2}}{f} a_{x}+\frac{\omega k}{f} a_{t}+12 i \omega \frac{\kappa}{r} V^{\prime} v_{y}=0 \\
& f a_{y}^{\prime \prime}+\left(f^{\prime}+\frac{f}{r}\right) a_{y}^{\prime}+\left(\frac{\omega^{2}}{f}-\frac{k^{2}}{r^{2}}\right) a_{y}+12 i k \frac{\kappa}{r} \phi^{\prime} v_{z}+12 i k \frac{\kappa}{r} K^{\prime} a_{z}- \\
& 12 i \omega \frac{\kappa}{r} V^{\prime} v_{x}-12 i k \frac{\kappa}{r} V^{\prime} v_{t}=0 \\
& f a_{z}^{\prime \prime}+\left(f^{\prime}+\frac{f}{r}\right) a_{z}^{\prime}+\left(\frac{\omega^{2}}{f}-\frac{k^{2}}{r^{2}}\right) a_{z}-12 i k \frac{\kappa}{r} \phi^{\prime} v_{y}=0
\end{aligned}
$$

And the constraints

$$
\begin{aligned}
\frac{i \omega}{f} a_{t}^{\prime}+\frac{i k}{r^{2}} a_{x}^{\prime} & =0 \\
\frac{i \omega}{f} v_{t}^{\prime}+\frac{i k}{r^{2}} v_{x}^{\prime}+2 \psi^{\prime} \delta-2 \psi \delta^{\prime} & =0
\end{aligned}
$$

Where $a_{i}$ and $v_{i}$ are the perturbations of the axial and vector gauge fields respectively. $\rho$ and $\delta$ are the real and imaginary parts of the perturbations of the scalar field, respectively. Momentum points in the $x$-direction, whereas the superfluid velocity points in the $z$ direction. 


\section{Chapter 13}

\section{General conclusions and outlook of the work}

The original content of this thesis is presented in Parts 2, 3 and 4. Parts 2 and 3 have well differenciated topics that somehow merge to give rise to the discussion put forward in Part 4.

Summary of Part 2 We have analyzed anomalous transport coefficients at strong coupling by considering extensions to the bulk action that account for both the chiral and mixed gravitational anomaly. This implies that the boundary current satisfies the expected anomalous conservation law

$$
\nabla_{\mu} J_{a}^{\mu}=\epsilon^{\mu \nu \rho \lambda}\left(\frac{d_{a b c}}{32 p i^{2}} F_{\mu \nu}^{b} F_{\rho \lambda}^{c}+\frac{b_{a}}{768 \pi^{2}} R_{\beta \mu \nu}^{\alpha} R_{\alpha \rho \lambda}^{\beta}\right)
$$

being $d_{a b c}=\frac{1}{2} \operatorname{tr}\left(T_{a}\left\{T_{b}, T_{c}\right\}\right)_{L}-(L \leftrightarrow R)$ and $b_{a}=\operatorname{tr}\left(\left(T_{a}\right)_{L}-(L \leftrightarrow R)\right.$ the anomaly coefficients. These are implemented phenomenologically by $\kappa$ and $\lambda$ coefficients respectively, appearing in (5.1.6.).

Using the corresponding bottom-up model we have calculated all the anomalous conductivities in an ordinary (i.e. non-superconducting) medium at finite temperature and density, showing on the one hand that the mixed anomaly is indeed responsible for the appearance of the $T^{2}$ contribution to the CVC and, on the other, that chiral transport coefficients at strong coupling have the same value than their weakly coupling counterparts, namely

$$
\begin{array}{rlrl}
\sigma_{V V}^{\mathcal{B}} \equiv \sigma_{C M E} & =\frac{\mu_{5}}{2 \pi^{2}} ; & \sigma_{A V}^{\mathcal{B}} \equiv \sigma_{C S E}=\frac{\mu}{2 \pi^{2}}, \\
\sigma_{V}^{\mathcal{V}}=\frac{\mu \mu_{5}}{2 \pi^{2}} ; & \sigma_{A}^{\mathcal{V}}=\frac{\mu_{5}^{2}+\mu^{2}}{4 \pi^{2}}+\frac{T^{2}}{12} .
\end{array}
$$

This latter statement turns out to be true only because we have non-dynamical gauge fields on the boundary; quantum corrections are expected otherwise.

We have also studied the cutoff flow of chiral conductivities by defining several theories equipped with a cutoff $\Lambda$. In order to do so we developed several methods and showed the equivalence of one to another. Analyzing the flow in the presence of the gravitational anomaly becomes problematic because one has to work with a higher derivative theory. 
After addressing all technical issues, we have obtained a cutoff flow of the form

$$
\begin{aligned}
\left\langle J^{x} J^{z}\right\rangle & =\frac{i k \kappa \mu\left(1-u_{c}\right)}{2 G \pi}=-i k \frac{\mu(\Lambda)}{4 \pi^{2}} \\
\left\langle J^{x} T_{t}^{z}\right\rangle & =-\frac{i k \kappa\left(1-u_{c}\right)^{2} \mu^{2}}{4 G \pi}-\frac{i k \lambda(-2+a)^{2} r_{H}^{2}}{2 G L^{4} \pi}=i k\left(\frac{\mu^{2}\left(1-u_{c}\right)^{2}}{8 \pi^{2}}+\frac{T^{2}}{24}\right) \\
\left\langle T_{t}^{x} J^{z}\right\rangle & =-\frac{i k \kappa\left(1-u_{c}\right)^{2} \mu^{2}}{4 G \pi}-\frac{i k \lambda(-2+a)^{2} r_{H}^{2}}{2 G L^{4} \pi}=i k\left(\frac{\mu^{2}\left(1-u_{c}\right)^{2}}{8 \pi^{2}}+\frac{T^{2}}{24}\right) \\
\left\langle T_{t}^{x} T_{t}^{z}\right\rangle & =\frac{i k \kappa\left(1-u_{c}\right)^{3} \mu^{3}}{6 G \pi}+\left(1-u_{c}\right) \mu \frac{i k \lambda(-2+a)^{2} r_{H}^{2}}{G L^{4} \pi}=-i k\left(\frac{\mu^{3}\left(1-u_{c}\right)^{3}}{12 \pi^{2}}+\frac{\mu(\Lambda) T^{2}}{12}\right)
\end{aligned}
$$

with $u_{c}=r_{H}^{2} / \Lambda^{2}\left(r_{H}\right.$ is the horizon radius), so that there exists an effective chemical potential $\mu(\Lambda)=\mu\left(1-r_{H}^{2} / \Lambda^{2}\right)$ and a non-flowing effective temperature $T$. The results can be interpreted as follows. We have a flowing chemical potential, flow that is easily interpretable in terms of the energy necessary to bring a unit of charge from the horizon to the boundary.

Summary of Part 3 We have studied several extensions to usual superfluids by means of holography. Holographic techniques make it simple to compute certain finite temperature quantities, such as conductivities or decay rates, which in turn are deeply afected by the fact that the dual theory is in a strong coupling regime.

We presented a holographic bottom-up model in which a $U(2)$ symmetry gets spontaneously broken to $U(1)$. This has the peculiarity of giving rise to non-relativistic massless modes in its spectrum, known as type II Nambu-Goldstone bosons, whose dispersion relation reads

$$
\omega=\mathcal{B} k^{2}-i \mathcal{C} k^{2}
$$

In our model type II Nambu-Goldstone bosons are well-defined quasi-particles at low temperatures (i.e. $\mathcal{C}<<\mathcal{B}$ ) and, contrary to the case of type I Goldstone bosons, their corresponding velocity of sound does not seem to take any universal value at vanishing temperature. Moreover, it has been shown recently that type II Goldstone bosons are accompanied by a massive partner mode whose gap is universal and given by

$$
\omega=2 \mu+\mathcal{O}\left(k^{2}\right) .
$$

Even though the computation of this gap is computationally more demanding, we have been able to shown explicitly that even at strong coupling the value of such a mass corresponds to the one predicted theoretically. Within the spectrum of the usual $U(1)$ holographic superconductor there is only one, purely imaginary, diffusive mode that depends on temperature; this happens to have important consequences for the late-time response of the system to certain perturbations: the perturbation could end up being oscillatory or being exponentially suppressed at leading order, depending on temperature. In our $U(2)$ model, we observed that diffusive modes come in pairs have a real part in their dispersion relation. Hence the aforementioned behaviour at late times is always oscillatory. Conductivities in this model become infinite at low frequencies, signaling the presence of superconductivity. We also worked out a different model that features a $U(2)$ global symmetry in the bulk, which we call ungauged. The ungauged model does not have an operator realization of some of the charges in the boundary theory and therefore the Goldstone's 
theorem does not apply to it, which implies that we did not have an a-priori expectation on the spectrum of excitations. Remarkably, we show that the ungauged model still presents type II Goldstone mode in its spectrum.

According to the Landau Criterion of superfluidity, a system featuring type II NambuGoldstone bosons in its spectrum cannot superfluid. The reason is that its critical velocity, defined as

$$
v_{\max }=\min \frac{\epsilon(\vec{k})}{k},
$$

vanishes. This fact entails that any finite supervelocity will drive the system out of the superfluid phase. We studied the Landau Criterion at strong coupling by means of our gauged and ungauged models, finding that the system gets perturbatively unstable at certain critical velocity, in accordance with Landau's ideas. We observe that the modes that induce the instability are precisely the Nambu-Goldstone bosons. When the model only has type I Goldstone particles (whose dispersion relation is linear) the critical velocity is finite and depends on temperature. However, for the gauged and ungauged models we find that $v_{\max }=0$ for any superfluid velocity. Both behaviours are expected in view of the validity of 13.0.9). We conclude that the Landau Criterion is fulfilled at strong coupling, in our holographic model(s).

Finally, we have constructed a s+p-wave superfluid numerically in holography. This phase is characterized by the coexistence of two order parameters. One of the is a scalar (swave) and the other one is a vector (p-wave). These sort of superfluids are less frequent in nature, even though some modified version of the $\mathrm{s}+\mathrm{p}$ superfluid is expected to be realized in real systems. One can reach this phase from the gauged $U(2)$ model that we commented on before; we observed the $U(2)$ s-wave superfluid presents a further instability at low temperatures (around $0.4 T_{c}$ ) towards this $\mathrm{s}+\mathrm{p}$ superfluid. We have also turned on an aditional chemical potential (making two of them different from zero in total) and studied the phase diagram of the resulting unbalanced $\mathrm{s}+\mathrm{p}$-superfluid, reaching interesting conclusions, such as the possibility for the existence of a Quatum Phase Transition.

Summary of Part 4 This last part has been distinguished from the rest for it acts as a combination of Parts 2 and 3. In it we discuss the form of anomalous transport coefficients for a (usual $U(1)$ s-wave) superfluid at finite temperature and density. Here the analysis is restricted to the chiral anomaly for the implementation of the mixed anomaly induce serious complications. Apart from the Chiral Magnetic and Vortical Conductivities, present already in ordinary fluids, we compute the so-called Chiral Electric Conductivity and point out the physical existence of a charge "generation" in the presence of a supervelocity aligned with an external magnetic field

$$
\rho=\hat{\sigma} \vec{\xi} \cdot \vec{B}
$$

We call this effect Chiral Charge Generation Effect and Chiral Charge Generation Conductivity its corresponding transport coefficient, $\hat{\sigma}$.

We study two models. In the first one we implement a AAA anomaly and the anomalous $U(1)$ symmetry gets spontaneously broken. We show that all anomalous conductivities tend to universal values at zero temperature, even though at finite temperature their values are not universal. At zero temperature one gets

$$
\begin{array}{r}
\sigma_{55}(T \rightarrow 0) \approx \frac{8 \kappa}{3} \mu_{5}=\frac{\sigma_{55}\left(T_{c}\right)}{3}, \\
\sigma_{C E E}(T \rightarrow 0) \approx \sigma_{C C G E}(T \rightarrow 0) \approx \frac{8 \kappa}{3} \xi_{x}
\end{array}
$$


where $\kappa \propto C$ is the anomaly coefficient, $\mu_{5}$ the (axial) chemical potential and $\xi_{x}$ the supervelocity. Note that 13.0.11) is independent from the superfluid velocity.

We also analyze a more sophisticated situation in which we start with a $U(1)_{V} \times U(1)_{A}$ symmetry ( $V$ stands for "vector" and $A$ for "axial"). There is a wider set of chiral conductivities that one can study in this setup once the vector symmetry gets spontaneously broken, and we compute all of them. At zero temperature we observe again that their value becomes universal and given by

$$
\begin{aligned}
& \sigma_{C S E}(T \rightarrow 0) \approx \frac{6 \kappa}{2} \mu=\frac{\sigma_{C S E}\left(T_{c}\right)}{2}, \\
& \sigma_{C^{2} G E}^{(V, A)}(T \rightarrow 0) \approx 3 \kappa \xi_{z} ; \quad \sigma_{C E E}^{(V, A)}(T \rightarrow 0) \approx 3 \kappa \xi_{x} .
\end{aligned}
$$

Equations 13.0.11)-13.0.14 represent different types of chiral conductivities, but they all have in common the universality of the coefficient in front of the thermodynamic parameter. In fact, the value itself is not actually important for what we call $\kappa$ could be different in the AAA and AVV models. The important measure of the correction is the relation between the conductivities in the unbroken and unbroken phases at zero temperature. This is, schematically

$$
\begin{array}{ll}
A A A: & \text { Broken }=\frac{1}{3} \text { Unbroken }, \\
A V V: & \text { Broken }=\frac{1}{2} \text { Unbroken },
\end{array}
$$

as indicated in equations (13.0.11) and (13.0.13). So we conclude that chiral conductivities in superfluids become universal at zero temperature and they suffer a correction which depends solely on the structure of broken symmetries and its relation to the type of anomaly. 


\section{Chapter 14}

\section{Conclusiones generales del trabajo}

El contenido original de esta tesis se presenta en las partes 2,3 y 4 . Las partes 2 y 3 tienen temas bien diferenciadas que se unen de alguna manera para dar lugar a la discusión presentada en la Parte 4 .

Resumen de la Parte 1 Hemos explicado el contenido no original de este trabajo, que sirve como prefacio para entender los contenidos que se presentan en las Partes 2, 3 y 4.

Empezamos dando una visión global y bastante práctica de la dualidad AdS/CFT, poniendo énfasis en las aplicaciones a la física de la materia condensada. Se explican las nociones básicas y el diccionario holográfico. También se discute cómo implementar una densidad de carga finita y se hace una breve introducción al tema de superconductores holográficos, introducción que será ampliada en el capítulo correspondiente a ese tema. Se tratan también las técnicas teóricas disponibles para tratar sistemas físicos fuera del equilibrio mediante la correspondencia AdS/CFT, que se pueden resumir en dos aproximaciones: Hidrodinámica y Teoría de Respuesta Lineal. También se comenta brevemente acerca de la región en el espacio de parámetros en que ambas aproximaciones tienen validez.

Se pasa luego al estudio de los llamados coeficientes de transporte anómalos, haciendo hincapié en el régimen de acoplamiendo débil. Se presenta la manera en la que dichos coeficientes son entendidos tanto desde el punto de vista hidrodinámico como a través de las fórmulas de Kubo.

Por último se incluye una pequeña introducción al concepto de superfluido, al Criterio de Landau y al efecto Meissner, así como a la descripción hidrodinámica de un superfluido.

Resumen de la Parte 2 Hemos analizado los coeficientes de transporte anómalos en acoplamiento fuerte tras incluir en la acción en el bulk los términos que dan lugar tanto a la anomalía quiral y como la anomalía mixta. Esto implica que la corriente satisface la ley de (no) conservación esperada debido a la anomalía

$$
\nabla_{\mu} J_{a}^{\mu}=\epsilon^{\mu \nu \rho \lambda}\left(\frac{d_{a b c}}{32 p i^{2}} F_{\mu \nu}^{b} F_{\rho \lambda}^{c}+\frac{b_{a}}{768 \pi^{2}} R_{\beta \mu \nu}^{\alpha} R_{\alpha \rho \lambda}^{\beta}\right)
$$

siendo $d_{a b c}=\frac{1}{2} \operatorname{tr}\left(T_{a}\left\{T_{b}, T_{c}\right\}\right)_{L}-(L \leftrightarrow R)$ y $b_{a}=\operatorname{tr}\left(\left(T_{a}\right)_{L}-(L \leftrightarrow R)\right.$ los coeficientes de las anomalías. Éstos se implementan fenomenológicamente a través de los coeficientes $\kappa \mathrm{y}$ $\lambda$ respectivamente, que aparecen en (5.1.6). Usando el modelo bottom-up correspondiente hemos calculado todas las conductividades anómalas en un medio ordinario (es decir, no 
superconductor) a temperatura y la densidad finitas, lo que muestra por una parte que la anomalía mixta es de hecho responsable de la aparición de contributción del tipo $T^{2}$ a la Conductivitdad Quiral Vortical y ,por otro, que los coeficientes de transporte quiral en régimen de acoplamiento fuerte tienen el mismo valor que los correspondientes calculados en acoplamiento débil, a saber,

$$
\begin{array}{rlrl}
\sigma_{V V}^{\mathcal{B}} \equiv \sigma_{C M E} & =\frac{\mu_{5}}{2 \pi^{2}} ; & \sigma_{A V}^{\mathcal{B}} \equiv \sigma_{C S E}=\frac{\mu}{2 \pi^{2}}, \\
\sigma_{V}^{\mathcal{V}}=\frac{\mu \mu_{5}}{2 \pi^{2}} ; & \sigma_{A}^{\mathcal{V}}=\frac{\mu_{5}^{2}+\mu^{2}}{4 \pi^{2}}+\frac{T^{2}}{12} .
\end{array}
$$

Esta última afirmación resulta ser cierta sólo porque estamos considerando campos de gauge no dinámicos en la frontera; en el caso de que esto no fuese así, sería de esperar la aparición de correcciones cuánticas.

También hemos estudiado el flujo de conductividades quirales como función una escala de corte energética mediante la definición de varias teorías equipados con dicha escala que llamamos $\Lambda$. Para ello hemos desarrollado varios métodos y demostrado la equivalencia entre ellos. Analizar el flujo en presencia de la anomalía gravitacional se convierte en problemático debido a que es necesario trabajar con una teoría en derivadas superiores. Después de abordar todas las cuestiones técnicas, se ha obtenido un flujo en función de $\Lambda$ de la forma

$$
\begin{aligned}
\left\langle J^{x} J^{z}\right\rangle & =\frac{i k \kappa \mu\left(1-u_{c}\right)}{2 G \pi}=-i k \frac{\mu(\Lambda)}{4 \pi^{2}} \\
\left\langle J^{x} T_{t}^{z}\right\rangle & =-\frac{i k \kappa\left(1-u_{c}\right)^{2} \mu^{2}}{4 G \pi}-\frac{i k \lambda(-2+a)^{2} r_{H}^{2}}{2 G L^{4} \pi}=i k\left(\frac{\mu^{2}\left(1-u_{c}\right)^{2}}{8 \pi^{2}}+\frac{T^{2}}{24}\right) \\
\left\langle T_{t}^{x} J^{z}\right\rangle & =-\frac{i k \kappa\left(1-u_{c}\right)^{2} \mu^{2}}{4 G \pi}-\frac{i k \lambda(-2+a)^{2} r_{H}^{2}}{2 G L^{4} \pi}=i k\left(\frac{\mu^{2}\left(1-u_{c}\right)^{2}}{8 \pi^{2}}+\frac{T^{2}}{24}\right) \\
\left\langle T_{t}^{x} T_{t}^{z}\right\rangle & =\frac{i k \kappa\left(1-u_{c}\right)^{3} \mu^{3}}{6 G \pi}+\left(1-u_{c}\right) \mu \frac{i k \lambda(-2+a)^{2} r_{H}^{2}}{G L^{4} \pi}=-i k\left(\frac{\mu^{3}\left(1-u_{c}\right)^{3}}{12 \pi^{2}}+\frac{\mu(\Lambda) T^{2}}{12}\right)
\end{aligned}
$$

con $u_{c}=r_{H}^{2} / \Lambda^{2}\left(r_{H}\right.$ es el radio del horizonte), por lo que existe un potencial químico efectivo $\mu(\Lambda)=\mu\left(1-r_{H}^{2} / \Lambda^{2}\right)$ y una temperatura efectiva $T$ independiente de $\Lambda$. Los resultados se pueden interpretar como sigue: tenemos un potencial químico que fluye, flujo que es fácilmente interpretable en términos de la energía necesaria para llevar una unidad de carga desde el horizonte hasta la frontera del espacio-tiempo.

Resumen de la Parte 3 Hemos estudiado varios superfluidos no usuales por medio de las técnicas de Holografía. Éstas técnicas hacen sencillo calcular ciertas cantidades a temperatura finita, como conductividades o tasas de descomposición, las cuales a su vez están profundamente afectadas por el sector termalizado, que en la teoría dual se encuentra en un régimen de acoplamiento fuerte.

Se presenta un modelo bottom-up holográfico en el que una simetría $U(2)$ (modelo gauged en el bulk) se rompe espontáneamente a $U(1)$. Esto tiene la particularidad de dar lugar a modos sin masa no relativistas en su espectro conocidos como bosones de NambuGoldstone de tipo II, con una relación de dispersión

$$
\omega=\mathcal{B} k^{2}-i \mathcal{C} k^{2} .
$$

En nuestro modelo los bosones de Nambu-Goldstone de tipo II son cuasipartículas bien definidas a bajas temperaturas (es decir, $\mathcal{C}<<\mathcal{B}$ ) y, contrariamente a lo que ocurre para 
bosones de tipo I, su correspondiente velocidad del sonido no parece tener ningún valor universal a temperatura cero. Por otra parte, se ha demostrado recientemente que los bosones de Goldstone el tipo II aparecen junto a un modo compañero masivo cuya masa es universal y dada por ( $\mu$ es el potencial químico)

$$
\omega=2 \mu+\mathcal{O}\left(k^{2}\right) .
$$

A pesar de que el cálculo de esta masa es computacionalmente más exigente, hemos sido capaces de mostrar explícitamente que incluso en acoplamiento fuerte el valor de dicha masa se corresponde con la predicha teóricamente. En el superconductor holográfico $U(1)$ habitual sólo hay un modo difusivo, puramente imaginario, que depende de la temperatura; ésto tiene consecuencias importantes en la respuesta del sistema a ciertas perturbaciones a tiempos grandes: la perturbación podría acabar presentando un comportamiento oscilatorio o siendo exponencialmente suprimida, a primer orden, en función de la temperatura. En nuestro modelo $U(2)$ se observa que los modos difusivos vienen en pares y tienen una parte real en su relación de dispersión. Por lo tanto el comportamiento antes mencionado a tiempos largos siempre es oscilatorio en este modelo. Además, las conductividades se vuelven infinitas a frecuencias bajas, lo que indica la presencia de superconductividad. También hemos elaborado un modelo diferente que cuenta con una simetría global $U(2)$ en el bulk, el cual llamamos modelo ungauged. Este modelo hace que la teoría dual carezca de algunos operadores carga, por lo que el teorema de Goldstone no aplica. A su vez esto hace que no tengamos una expectativa a priori sobre el espectro de excitaciones. Sorprendentemente, obtenemos que el modelo ungauged presenta el modo Goldstone tipo II en su espectro.

De acuerdo con el criterio de superfluidez de Landau, un sistema con bosones de NambuGoldstone de tipo II en su espectro no puede superfluir. La razón es que su velocidad crítica, que se define como

$$
v_{\max }=\min \frac{\epsilon(\vec{k})}{k},
$$

es nula. Este hecho implica que cualquier supervelocidad no cero llevaría al sistema fuera de la fase de superfluido. En este trabajo hemos estudiado el Criterio de Landau en acoplamiento fuerte por medio de nuestros modelos $U(2)$ gauged y ungauged, encontrando que el sistema se vuelve inestable perturbativamente a cierta velocidad crítica, en conformidad con las ideas de Landau. Observamos que los modos que inducen la inestabilidad son, precisamente, los bosones de Nambu-Goldstone. Cuando el modelo sólo tiene el tipo I de partículas de Goldstone (cuya relación de dispersión es lineal) la velocidad crítica es no nula y depende de la temperatura. Sin embargo, para los modelos gauged y ungauged, que como se ha explicado contienen bosones de Goldstone de tipo II (con una relación de dispersión cuadrática 14.0.7) ),encontramos que $v_{\max }=0$ para cualquier supervelocidad. Ambos comportamientos son esperables en vista de la validez de (14.0.9). Llegamos así a la conclusión de que el criterio de Landau se cumple a acoplamiento fuerte en nuestro(s) modelo(s) holográfico(s).

Por último, hemos construido un superfluido $\mathrm{s}+\mathrm{p}$ numéricamente en Holografía. Esta fase se caracteriza por la coexistencia de dos parámetros de orden. Uno de los es un escalar (onda tipo $s$ ) y el otro es un vector (onda tipo $p$ ). Este tipo de superfluidos son menos frecuentes en la naturaleza, aunque alguna versión modificada del superfluido s+p se esperaría poder realizar en sistemas reales. Uno puede llegar a esta fase a partir de modelo $U(2)$ gauged sobre el que comentamos antes; observamos que el superfluido $U(2)$ de tipo $s$ presenta una inestabilidad adicional a bajas temperaturas (alrededor de $0.4 T_{c}$ ) en dirección a este $\mathrm{s}+\mathrm{p}$ superfluido. También hemos encendido un potencial químico adicional 
(haciendo en total dos de ellos diferentes de cero) y estudiado el diagrama de fases del resultante superfluido $\mathrm{s}+\mathrm{p}$ desequilibrado, llegando a conclusiones interesantes, como la posibilidad de la existencia de una transición de fase cuántica.

Resumen de la Parte 4 Esta última parte se ha distinguido del resto porque actúa como una combinación de las partes 2 y 3 . En ella se discute la forma de los coeficientes de transporte anómalos para un superfluido $(U(1)$ en onda $s$ ) a temperatura y densidad finitas. El análisis se restringe a la anomalía quiral ya que la inclusión de la anomalía mixta induce complicaciones importantes. Aparte de la Conductividad Quiral Magnética y Conductividad Quiral Vortical, presentes ya en líquidos ordinarios, calculamos la llamada Conductividad Quiral Eléctrica y señalamos la existencia física de una "generación" de carga en presencia de una supervelocidad alineada con un campo magnético externo

$$
\rho=\hat{\sigma} \vec{\xi} \cdot \vec{B}
$$

Llamamos a este efecto Efecto Quiral de Generación de carga y a su correspondiente conductividad Conductividad de Generación Quiral de Carga ( $\hat{\sigma}$ en la ecuación) .

Se estudian dos modelos. En el primero implementamos una anomalía AAA y la simetría $U(1)$ anómala se rompe espontáneamente. Se demuestra que todas las conductividades anómalas tienden a valores universales a temperatura cero, a pesar de que a temperatura finita sus valores no son universales. A temperatura cero se obtiene

$$
\begin{array}{r}
\sigma_{55}(T \rightarrow 0) \approx \frac{8 \kappa}{3} \mu_{5}=\frac{\sigma_{55}\left(T_{c}\right)}{3}, \\
\sigma_{C E E}(T \rightarrow 0) \approx \sigma_{C C G E}(T \rightarrow 0) \approx \frac{8 \kappa}{3} \xi_{x},
\end{array}
$$

donde $\kappa \propto C$ es el coeficiente de la anomalía , $\mu_{5}$ el potencial químico (axial) y $\xi_{x}$ la supervelocidad . Notar que (14.0.11) es independiente de la supervelocidad.

También analizamos una situación más sofisticada en la que comenzamos con una simetría $U(1)_{V} \times U(1)_{A}(V$ significa "vector" y $A$ significa "axial" $)$. Hay un conjunto más amplio de conductividades quirales que se pueden estudiar en esta situación una vez que la simetría vectorial se rompe espontáneamente, y calculamos todos ellos. A temperatura cero se observa una vez más que su valor se convierte en universal y dado por

$$
\begin{aligned}
& \sigma_{C S E}(T \rightarrow 0) \approx \frac{6 \kappa}{2} \mu=\frac{\sigma_{C S E}\left(T_{c}\right)}{2}, \\
& \sigma_{C^{2} G E}^{(V, A)}(T \rightarrow 0) \approx 3 \kappa \xi_{z} ; \quad \sigma_{C E E}^{(V, A)}(T \rightarrow 0) \approx 3 \kappa \xi_{x} .
\end{aligned}
$$

Las ecuaciones 14.0.11)-14.0.14 representan diferentes tipos de conductividades quirales, pero todas tienen en común la universalidad del coeficiente que multiplica al parámetro termodinámico. De hecho, el valor en sí mismo no es realmente importante porque lo que llamamos $\kappa$ podría ser diferente en los modelos AAA y AVV . La medida importante de la corrección es la relación entre la conductividad entre las fases ordinaria (no rota) y espontáneamente rota a temperatura cero. Esto es, esquemáticamente

$$
\begin{array}{ll}
A A A: & \sigma(\text { Rota })=\frac{1}{3} \sigma(\text { No rota }), \\
A V V: & \sigma(\text { Rota })=\frac{1}{2} \sigma(\text { No rota }),
\end{array}
$$

como se indica en las ecuaciones 14.0.11 y 14.0.13). Por lo tanto concluimos que las conductividades quirales en superfluidos se convierten en universales a temperatura cero y sufren una corrección que depende únicamente de la estructura de simetrías rotas y su relación con el tipo de anomalía. 


\section{Bibliography}

[1] V. E. Hubeny and M. Rangamani, A Holographic view on physics out of equilibrium, Adv.High Energy Phys. 2010 (2010) 297916, [1006.3675].

[2] I. Amado, M. Kaminski, and K. Landsteiner, Hydrodynamics of Holographic Superconductors, JHEP 0905 (2009) 021, arXiv:0903.2209.

[3] S. A. Hartnoll, C. P. Herzog, and G. T. Horowitz, Holographic Superconductors, JHEP 0812 (2008) 015, 0810.1563.

[4] I. Amado, N. Lisker, and A. Yarom, Universal chiral conductivities for low temperature holographic superfluids, 1401.5795.

[5] J. M. Maldacena, The large $N$ limit of superconformal field theories and supergravity, Adv. Theor. Math. Phys. 2 (1998) 231-252, hep-th/9711200.

[6] S. Gubser, I. R. Klebanov, and A. M. Polyakov, Gauge theory correlators from noncritical string theory, Phys.Lett. B428 (1998) 105-114, hep-th/9802109.

[7] E. Witten, Anti-de Sitter space and holography, Adv.Theor.Math.Phys. 2 (1998) 253-291, hep-th/9802150.

[8] G. 't Hooft, Dimensional reduction in quantum gravity, gr-qc/9310026.

[9] L. Susskind, The World as a hologram, J.Math.Phys. 36 (1995) 6377-6396, hep-th/9409089.

[10] G. 't Hooft, A Planar Diagram Theory for Strong Interactions, Nucl.Phys. B72 (1974) 461.

[11] J. M. Bardeen, B. Carter, and S. Hawking, The Four laws of black hole mechanics, Commun.Math.Phys. 31 (1973) 161-170.

[12] J. D. Brown and M. Henneaux, Central Charges in the Canonical Realization of Asymptotic Symmetries: An Example from Three-Dimensional Gravity, Commun.Math.Phys. 104 (1986) 207-226.

[13] A. Strominger, Black hole entropy from near horizon microstates, JHEP 9802 (1998) 009, hep-th/9712251.

[14] E. D'Hoker and D. Z. Freedman, Supersymmetric gauge theories and the AdS / CFT correspondence, hep-th/0201253.

[15] O. Aharony, S. S. Gubser, J. M. Maldacena, H. Ooguri, and Y. Oz, Large $N$ field theories, string theory and gravity, Phys. Rept. 323 (2000) 183-386, hep-th/9905111. 
[16] E. T. Akhmedov, Introduction to the AdS / CFT correspondence, hep-th/9911095.

[17] I. R. Klebanov, TASI lectures: Introduction to the AdS / CFT correspondence, hep-th/0009139.

[18] H. Nastase, Introduction to AdS-CFT, 0712.0689.

[19] J. Polchinski, Introduction to Gauge/Gravity Duality, 1010.6134

[20] K. Skenderis, Lecture notes on holographic renormalization, Class.Quant.Grav. 19 (2002) 5849-5876, hep-th/0209067.

[21] I. Papadimitriou and K. Skenderis, AdS / CFT correspondence and geometry, hep-th/0404176.

[22] I. Papadimitriou, Holographic renormalization as a canonical transformation, JHEP 1011 (2010) 014, 1007.4592.

[23] I. Papadimitriou, Multi-Trace Deformations in AdS/CFT: Exploring the Vacuum Structure of the Deformed CFT, JHEP 0705 (2007) 075, hep-th/0703152.

[24] J. McGreevy, Holographic duality with a view toward many-body physics, Adv.High Energy Phys. 2010 (2010) 723105, [0909.0518.

[25] A. G. Green, An Introduction to Gauge Gravity Duality and Its Application in Condensed Matter, Contemp.Phys. 54 (2013), no. 1 33, 1304.5908.

[26] S. A. Hartnoll, Lectures on holographic methods for condensed matter physics, Class.Quant.Grav. 26 (2009) 224002, 0903.3246.

[27] S. A. Hartnoll, Horizons, holography and condensed matter, 1106.4324.

[28] T. K. Misner, C.W. and J. Wheeler, Gravitation. Freeman, W.H., 1973.

[29] S. Hawking and D. N. Page, Thermodynamics of Black Holes in anti-De Sitter Space, Commun.Math.Phys. 87 (1983) 577.

[30] L. Landau and E. Lifshitz, Course of Theoretical Physcis, Vol. 6. Elsevier.

[31] N. Andersson and G. Comer, Relativistic fluid dynamics: Physics for many different scales, Living Rev.Rel. 10 (2007) 1, gr-qc/0605010.

[32] C. Eckart, The Thermodynamics of irreversible processes. 3.. Relativistic theory of the simple fluid, Phys.Rev. 58 (1940) 919-924.

[33] P. Kovtun, Lectures on hydrodynamic fluctuations in relativistic theories, J.Phys. A45 (2012) 473001, 1205.5040.

[34] D. Nickel and D. T. Son, Deconstructing holographic liquids, New J.Phys. 13 (2011) 075010, 1009.3094.

[35] S. Endlich, A. Nicolis, R. A. Porto, and J. Wang, Dissipation in the effective field theory for hydrodynamics: First order effects, Phys.Rev. D88 (2013) 105001, 1211.6461 .

[36] P. Kovtun, D. T. Son, and A. O. Starinets, Viscosity in strongly interacting quantum field theories from black hole physics, Phys.Rev.Lett. 94 (2005) 111601, hep-th/0405231. 
[37] K. S. Thorne, R. Price, and D. Macdonald, BLACK HOLES: THE MEMBRANE PARADIGM, .

[38] S. Bhattacharyya, V. E. Hubeny, S. Minwalla, and M. Rangamani, Nonlinear Fluid Dynamics from Gravity, JHEP 0802 (2008) 045, [0712.2456].

[39] M. Rangamani, Gravity and Hydrodynamics: Lectures on the fluid-gravity correspondence, Class.Quant.Grav. 26 (2009) 224003, 0905.4352.

[40] D. Forster, Hydrodynamic Fluctuations, Broken Symmetry, And Correlation Functions. Advanced Books Classics.

[41] D. E. Kharzeev and H.-U. Yee, Chiral Magnetic Wave, Phys.Rev. D83 (2011) 085007, 1012.6026.

[42] A. Das, Finite Temperature Field Theory. World Scientific.

[43] N. Landsman and C. van Weert, Real and Imaginary Time Field Theory at Finite Temperature and Density, Phys.Rept. 145 (1987) 141.

[44] D. T. Son and A. O. Starinets, Minkowski space correlators in AdS / CFT correspondence: Recipe and applications, JHEP 0209 (2002) 042, hep-th/0205051.

[45] C. P. Herzog and D. T. Son, Schwinger-Keldysh propagators from AdS/CFT correspondence, JHEP 03 (2003) 046, hep-th/0212072.

[46] M. Stephanov and Y. Yin, Conductivity and quasinormal modes in holographic theories, JHEP 1202 (2012) 017, 1111.5303.

[47] G. T. Horowitz and V. E. Hubeny, Quasinormal modes of AdS black holes and the approach to thermal equilibrium, Phys.Rev. D62 (2000) 024027, hep-th/9909056.

[48] D. Birmingham, I. Sachs, and S. N. Solodukhin, Conformal field theory interpretation of black hole quasinormal modes, Phys.Rev.Lett. 88 (2002) 151301, hep-th/0112055.

[49] E. Berti, V. Cardoso, and A. O. Starinets, Quasinormal modes of black holes and black branes, Class.Quant.Grav. 26 (2009) 163001, 0905.2975.

[50] K. Landsteiner, The Sound of Strongly Coupled Field Theories: Quasinormal Modes In AdS, AIP Conf.Proc. 1458 (2011) 174-189, 1202.3550.

[51] M. Kaminski, K. Landsteiner, J. Mas, J. P. Shock, and J. Tarrio, Holographic Operator Mixing and Quasinormal Modes on the Brane, JHEP 1002 (2010) 021, 0911.3610 .

[52] P. K. Kovtun and A. O. Starinets, Quasinormal modes and holography, Phys.Rev. D72 (2005) 086009, hep-th/0506184.

[53] S. L. Adler, Axial vector vertex in spinor electrodynamics, Phys.Rev. 177 (1969) 2426-2438.

[54] R. Delbourgo and A. Salam, The gravitational correction to pcac, Phys.Lett. B40 (1972) 381-382. 
[55] R. A. Bertlmann, Anomalies in quantum field theory, . Oxford, UK: Clarendon (1996) 566 p. (International series of monographs on physics: 91).

[56] A. Bilal, Lectures on Anomalies, 0802.0634.

[57] J. A. Harvey, TASI 2003 lectures on anomalies, hep-th/0509097.

[58] D. T. Son and A. R. Zhitnitsky, Quantum anomalies in dense matter, Phys. Rev. D70 (2004) 074018, hep-ph/0405216.

[59] A. Vilenkin, Parity nonconservation and neutrino transport in magnetic fields, Astrophys.J. 451 (1995) 700-702.

[60] A. Vilenkin, Cancellation of equilibrium parity violating currents, Phys.Rev. D22 (1980) 3067-3079.

[61] A. Vilenkin, Quntum field theory at finite temperature in a rotating system, Phys.Rev. D21 (1980) 2260-2269.

[62] A. Vilenkin, Parity Violating Currents in Thermal Radiation, Phys.Lett. B80 (1978) 150-152.

[63] M. A. Metlitski and A. R. Zhitnitsky, Anomalous axion interactions and topological currents in dense matter, Phys. Rev. D72 (2005) 045011, hep-ph/0505072.

[64] D. E. Kharzeev, L. D. McLerran, and H. J. Warringa, The effects of topological charge change in heavy ion collisions: 'Event by event $P$ and CP violation', Nucl. Phys. A803 (2008) 227-253, 0711.0950.

[65] D. Kharzeev, Parity violation in hot QCD: Why it can happen, and how to look for it, Phys. Lett. B633 (2006) 260-264, hep-ph/0406125.

[66] D. Kharzeev and A. Zhitnitsky, Charge separation induced by P-odd bubbles in QCD matter, Nucl. Phys. A797 (2007) 67-79, 0706.1026.

[67] D. E. Kharzeev and H. J. Warringa, Chiral Magnetic conductivity, Phys. Rev. D80 (2009) 034028, 0907.5007.

[68] K. Fukushima, D. E. Kharzeev, and H. J. Warringa, The Chiral Magnetic Effect, Phys. Rev. D78 (2008) 074033, 0808.3382.

[69] I. Amado, K. Landsteiner, and F. Pena-Benitez, Anomalous transport coefficients from Kubo formulas in Holography, JHEP 1105 (2011) 081, 1102.4577.

[70] K. Landsteiner, E. Megias, and F. Pena-Benitez, Gravitational Anomaly and Transport, Phys. Rev. Lett. 107 (2011) 021601, 1103.5006.

[71] F. Pena-Benitez, Anomaly induced transport coefficients, from weak to strong coupling, 1307.0540 .

[72] W. A. Bardeen and B. Zumino, Consistent and Covariant Anomalies in Gauge and Gravitational Theories, Nucl.Phys. B244 (1984) 421.

[73] J. Wess and B. Zumino, Consequences of anomalous Ward identities, Phys.Lett. B37 (1971) 95. 
[74] D. E. Kharzeev and H.-U. Yee, Anomalies and time reversal invariance in relativistic hydrodynamics: the second order and higher dimensional formulations, 1105.6360 .

[75] D. T. Son and P. Surowka, Hydrodynamics with Triangle Anomalies, Phys. Rev. Lett. 103 (2009) 191601, 0906.5044.

[76] Y. Neiman and Y. Oz, Relativistic Hydrodynamics with General Anomalous Charges, JHEP 03 (2011) 023, 1011.5107.

[77] K. Jensen, Triangle Anomalies, Thermodynamics, and Hydrodynamics, Phys.Rev. D85 (2012) 125017, 1203.3599.

[78] N. Banerjee, J. Bhattacharya, S. Bhattacharyya, S. Jain, S. Minwalla, et. al., Constraints on Fluid Dynamics from Equilibrium Partition Functions, JHEP 1209 (2012) 046, 1203.3544.

[79] A. V. Sadofyev and M. V. Isachenkov, The chiral magnetic effect in hydrodynamical approach, Phys. Lett. B697 (2011) 404-406, [1010.1550].

[80] F. M. Haehl, R. Loganayagam, and M. Rangamani, Effective actions for anomalous hydrodynamics, JHEP 1403 (2014) 034, 1312.0610.

[81] K. Jensen, R. Loganayagam, and A. Yarom, Thermodynamics, gravitational anomalies and cones, JHEP 1302 (2013) 088, 1207.5824.

[82] E. Megias and F. Pena-Benitez, Holographic Gravitational Anomaly in First and Second Order Hydrodynamics, JHEP 1305 (2013) 115, 1304.5529.

[83] S. Bhattacharyya, J. R. David, and S. Thakur, Second order transport from anomalies, JHEP 1401 (2014) 010, 1305.0340.

[84] G. M. Newman, Anomalous hydrodynamics, JHEP 01 (2006) 158, hep-ph/0511236.

[85] H.-U. Yee, Holographic Chiral Magnetic Conductivity, JHEP 11 (2009) 085, 0908.4189 .

[86] R. Loganayagam and P. Surowka, Anomaly/Transport in an Ideal Weyl gas, JHEP 1204 (2012) 097, 1201.2812.

[87] J. Erdmenger, M. Haack, M. Kaminski, and A. Yarom, Fluid dynamics of $R$-charged black holes, JHEP 01 (2009) 055, 0809.2488.

[88] N. Banerjee et. al., Hydrodynamics from charged black branes, JHEP 01 (2011) 094, 0809.2596.

[89] R. Loganayagam, Anomaly Induced Transport in Arbitrary Dimensions, 1106.0277.

[90] K. Jensen, Chiral anomalies and AdS/CMT in two dimensions, JHEP 1101 (2011) 109, 1012.4831.

[91] S. Jain and T. Sharma, Anomalous charged fluids in 1+1d from equilibrium partition function, JHEP 1301 (2013) 039, 1203.5308. 
[92] N. Banerjee, S. Dutta, S. Jain, R. Loganayagam, and T. Sharma, Constraints on Anomalous Fluid in Arbitrary Dimensions, JHEP 1303 (2013) 048, 1206.6499.

[93] L. Landau and E. M. Lifshitz, Course of Theoretical Physics: Volume 5. Elsevier 2008.

[94] D. Son, Low-energy quantum effective action for relativistic superfluids, hep-ph/0204199.

[95] A. Nicolis, Low-energy effective field theory for finite-temperature relativistic superfluids, 1108.2513 .

[96] S. Bhattacharyya, S. Jain, S. Minwalla, and T. Sharma, Constraints on Superfluid Hydrodynamics from Equilibrium Partition Functions, JHEP 1301 (2013) 040, 1206.6106.

[97] T. Albash and C. V. Johnson, A Holographic Superconductor in an External Magnetic Field, JHEP 0809 (2008) 121, 0804.3466.

[98] L. Tisza, Transport Phenomena in Helium II, Nature. 141 (3577) (1938) 913.

[99] I. Khalatnikov and V. Lebedev, Relativistic hydrodynamics of a superfluid liquid, Physics Letters A 91 (1982) 70.

[100] M. G. Alford, S. K. Mallavarapu, A. Schmitt, and S. Stetina, From a complex scalar field to the two-fluid picture of superfluidity, Phys.Rev. D87 (2013), no. 6 065001, 1212.0670.

[101] K. R. Atkins, Third and Fourth Sound in Liquid Helium II, Phys. Rev. 113 (Feb, 1959) 962-965.

[102] A. Yarom, Fourth sound of holographic superfluids, JHEP 0907 (2009) 070, 0903.1353 .

[103] C. Hoyos, B. S. Kim, and Y. Oz, Odd Parity Transport In Non-Abelian Superfluids From Symmetry Locking, 1404.7507.

[104] V. Gusynin, V. Miransky, and I. A. Shovkovy, Spontaneous rotational symmetry breaking and roton - like excitations in gauged sigma model at finite density, Phys.Lett. B581 (2004) 82-92, hep-ph/0311025.

[105] A. Nicolis, R. Rattazzi, and E. Trincherini, The Galileon as a local modification of gravity, Phys.Rev. D79 (2009) 064036, 0811.2197.

[106] T. Griffin, K. T. Grosvenor, P. Horava, and Z. Yan, Multicritical Symmetry Breaking and Naturalness of Slow Nambu-Goldstone Bosons, Phys.Rev. D88 (2013) 101701, 1308.5967.

[107] B. Halperin, Dynamic properties of the multicomponent Bose fluid, Phys. Rev. B 11 (1975) 178190.

[108] T. Schfer, D. Son, M. A. Stephanov, D. Toublan, and J. Verbaarschot, Kaon condensation and Goldstone's theorem, Phys.Lett. B522 (2001) 67-75, hep-ph/0108210. 
[109] V. Miransky and I. Shovkovy, Spontaneous symmetry breaking with abnormal number of Nambu-Goldstone bosons and kaon condensate, Phys.Rev.Lett. $\mathbf{8 8}$ (2002) 111601, hep-ph/0108178.

[110] T. Brauner, Spontaneous Symmetry Breaking and Nambu-Goldstone Bosons in Quantum Many-Body Systems, Symmetry 2 (2010) 609-657, 1001.5212.

[111] H. B. Nielsen and S. Chadha, On How to Count Goldstone Bosons, Nucl.Phys. B105 (1976) 445.

[112] H. Watanabe and T. Brauner, On the number of Nambu-Goldstone bosons and its relation to charge densities, Phys.Rev. D84 (2011) 125013, 1109.6327.

[113] H. Watanabe and H. Murayama, Unified Description of Nambu-Goldstone Bosons without Lorentz Invariance, Phys.Rev.Lett. 108 (2012) 251602, 1203.0609.

[114] Y. Hidaka, Counting rule for Nambu-Goldstone modes in nonrelativistic systems, Phys.Rev.Lett. 110 (2013) 091601, 1203.1494.

[115] A. Kapustin, Remarks on nonrelativistic Goldstone bosons, 1207.0457.

[116] H. Watanabe and H. Murayama, Redundancies in Nambu-Goldstone Bosons, Phys.Rev.Lett. 110 (2013) 181601, 1302.4800].

[117] T. Brauner, Spontaneous symmetry breaking in the linear sigma model at finite chemical potential: One-loop corrections, Phys.Rev. D74 (2006) 085010, hep-ph/0607102.

[118] A. Nicolis and F. Piazza, A relativistic non-relativistic Goldstone theorem: gapped Goldstones at finite charge density, Phys.Rev.Lett. 110 (2013) 011602, 1204.1570.

[119] R. Jackiw and S. Pi, Chern-Simons modification of general relativity, Phys.Rev. D68 (2003) 104012, gr-qc/0308071.

[120] S. Alexander and N. Yunes, Chern-Simons Modified General Relativity, Phys.Rept. 480 (2009) 1-55, 0907.2562.

[121] S. Golkar and D. T. Son, Non-Renormalization of the Chiral Vortical Effect Coefficient, 1207.5806 .

[122] D.-F. Hou, H. Liu, and H.-c. Ren, A Possible Higher Order Correction to the Vortical Conductivity in a Gauge Field Plasma, Phys.Rev. D86 (2012) 121703, 1210.0969.

[123] K. Jensen, P. Kovtun, and A. Ritz, Chiral conductivities and effective field theory, JHEP 1310 (2013) 186, [1307.3234].

[124] Y. Neiman and Y. Oz, Anomalies in Superfluids and a Chiral Electric Effect, 1106.3576 .

[125] L. Bonora, M. Cvitan, P. D. Prester, S. Pallua, and I. Smolic, Gravitational Chern-Simons Lagrangian terms and spherically symmetric spacetimes, 1105.4792.

[126] T. Delsate, V. Cardoso, and P. Pani, Anti de Sitter black holes and branes in dynamical Chern-Simons gravity: perturbations, stability and the hydrodynamic modes, JHEPA,1106,055.2011 1106 (2011) 055, [1103.5756. 
[127] V. Balasubramanian and P. Kraus, Space-time and the holographic renormalization group, Phys.Rev.Lett. 83 (1999) 3605-3608, hep-th/9903190.

[128] N. Iqbal and H. Liu, Universality of the hydrodynamic limit in AdS/CFT and the membrane paradigm, Phys.Rev. D79 (2009) 025023, 0809.3808.

[129] Y. Matsuo, S.-J. Sin, and Y. Zhou, Mixed RG Flows and Hydrodynamics at Finite Holographic Screen, JHEP 1201 (2012) 130, 1109.2698.

[130] B.-H. Lee, S. S. Pal, and S.-J. Sin, RG flow of transport quantities, Int.J.Mod.Phys. A27 (2012) 1250071, [1108.5577].

[131] X.-H. Ge, Y. Ling, Y. Tian, and X.-N. Wu, Holographic RG Flows and Transport Coefficients in Einstein-Gauss-Bonnet-Maxwell Theory, JHEP 1201 (2012) 117, 1112.0627.

[132] T. Faulkner, H. Liu, and M. Rangamani, Integrating out geometry: Holographic Wilsonian RG and the membrane paradigm, JHEP 1108 (2011) 051, 1010.4036.

[133] S. Grozdanov, Wilsonian Renormalisation and the Exact Cut-Off Scale from Holographic Duality, JHEP 1206 (2012) 079, 1112.3356.

[134] A. Rebhan, A. Schmitt, and S. A. Stricker, Anomalies and the chiral magnetic effect in the Sakai- Sugimoto model, JHEP 01 (2010) 026, 0909.4782.

[135] A. Gynther, K. Landsteiner, F. Pena-Benitez, and A. Rebhan, Holographic Anomalous Conductivities and the Chiral Magnetic Effect, JHEP 02 (2011) 110, 1005.2587.

[136] I. Heemskerk and J. Polchinski, Holographic and Wilsonian Renormalization Groups, JHEP 1106 (2011) 031, [1010.1264].

[137] I. Bredberg, C. Keeler, V. Lysov, and A. Strominger, Wilsonian Approach to Fluid/Gravity Duality, JHEP 1103 (2011) 141, 1006.1902.

[138] D. Martelli and W. Mueck, Holographic renormalization and Ward identities with the Hamilton-Jacobi method, Nucl.Phys. B654 (2003) 248-276, hep-th/0205061.

[139] O. Saremi and D. T. Son, Hall viscosity from gauge/gravity duality, 1103.4851.

[140] H.-U. Yee and I. Zahed, Holographic two dimensional QCD and Chern-Simons term, 1103.6286 .

[141] P. Kraus and F. Larsen, Holographic gravitational anomalies, JHEP 0601 (2006) 022, hep-th/0508218.

[142] D. Grumiller, R. B. Mann, and R. McNees, Dirichlet boundary value problem for Chern-Simons modified gravity, Phys.Rev. D78 (2008) 081502, 0803.1485.

[143] T. Clark, S. Love, and T. ter Veldhuis, Holographic Currents and Chern-Simons Terms, Phys.Rev. D82 (2010) 106004, 1006.2400.

[144] S. A. Hartnoll, C. P. Herzog, and G. T. Horowitz, Building a Holographic Superconductor, Phys.Rev.Lett. 101 (2008) 031601, 0803.3295.

[145] S. S. Gubser and S. S. Pufu, The Gravity dual of a p-wave superconductor, JHEP 0811 (2008) 033, 0805.2960]. 
[146] F. Benini, C. P. Herzog, R. Rahman, and A. Yarom, Gauge gravity duality for d-wave superconductors: prospects and challenges, JHEP 1011 (2010) 137, 1007.1981 .

[147] J.-W. Chen, Y.-J. Kao, D. Maity, W.-Y. Wen, and C.-P. Yeh, Towards A Holographic Model of D-Wave Superconductors, Phys.Rev. D81 (2010) 106008, 1003.2991.

[148] G. T. Horowitz, Introduction to Holographic Superconductors, Lect.Notes Phys. 828 (2011) 313-347, 1002.1722.

[149] C. P. Herzog, Lectures on Holographic Superfluidity and Superconductivity, J.Phys. A42 (2009) 343001, 0904.1975.

[150] I. R. Klebanov and E. Witten, AdS / CFT correspondence and symmetry breaking, Nucl.Phys. B556 (1999) 89-114, hep-th/9905104.

[151] S. S. Gubser, Breaking an Abelian gauge symmetry near a black hole horizon, Phys.Rev. D78 (2008) 065034, [0801.2977].

[152] M. Bhaseen, J. P. Gauntlett, B. Simons, J. Sonner, and T. Wiseman, Holographic Superfluids and the Dynamics of Symmetry Breaking, Phys.Rev.Lett. 110 (2013) 015301, 1207.4194.

[153] I. Amado, C. Hoyos-Badajoz, K. Landsteiner, and S. Montero, Hydrodynamics and beyond in the strongly coupled N=4 plasma, JHEP 0807 (2008) 133, 0805.2570.

[154] R. A. Davison and A. O. Starinets, Holographic zero sound at finite temperature, Phys.Rev. D85 (2012) 026004, [1109.6343].

[155] F. Bigazzi, A. L. Cotrone, D. Musso, N. P. Fokeeva, and D. Seminara, Unbalanced Holographic Superconductors and Spintronics, JHEP 1202 (2012) 078, 1111.6601.

[156] Z. J. Z. H. M. C. M. P. K. H. L. S. Z. Q. Li, E. A. Henriksen and D. N. Basov, Dirac charge dynamics in graphene by infrared spectroscopy, Nature Physics 4 (2008) $532-535$.

[157] I. Khalatnikov, An Introduction to the Theory of Superfluidity. Advanced Books Classics, Westview Press.

[158] D. Pines and P. Nozires, The Theory of Quantum Liquids. Advanced Book Classics. Perseus Books, 1999.

[159] C. Herzog, P. Kovtun, and D. Son, Holographic model of superfluidity, Phys.Rev. D79 (2009) 066002, 0809.4870.

[160] P. Basu, A. Mukherjee, and H.-H. Shieh, Supercurrent: Vector Hair for an AdS Black Hole, Phys.Rev. D79 (2009) 045010, 0809.4494.

[161] D. Arean, P. Basu, and C. Krishnan, The Many Phases of Holographic Superfluids, JHEP 1010 (2010) 006, 1006.5165.

[162] D. Arean, M. Bertolini, J. Evslin, and T. Prochazka, On Holographic Superconductors with DC Current, JHEP 1007 (2010) 060, 1003.5661. 
[163] P. Basu, J. He, A. Mukherjee, M. Rozali, and H.-H. Shieh, Competing Holographic Orders, JHEP 1010 (2010) 092, 1007.3480.

[164] R.-G. Cai, L. Li, L.-F. Li, and Y.-Q. Wang, Competition and Coexistence of Order Parameters in Holographic Multi-Band Superconductors, JHEP 1309 (2013) 074, 1307.2768.

[165] D. Musso, Competition/Enhancement of Two Probe Order Parameters in the Unbalanced Holographic Superconductor, JHEP 1306 (2013) 083, [1302.7205.

[166] M. G. Alford, S. K. Mallavarapu, A. Schmitt, and S. Stetina, Role reversal in first and second sound in a relativistic superfluid, Phys.Rev. D89 (2014) 085005, 1310.5953 .

[167] S. Nakamura, H. Ooguri, and C.-S. Park, Gravity Dual of Spatially Modulated Phase, Phys.Rev. D81 (2010) 044018, 0911.0679.

[168] A. Donos and J. P. Gauntlett, Holographic striped phases, JHEP 1108 (2011) 140, 1106.2004.

[169] C. B. Bayona, K. Peeters, and M. Zamaklar, A Non-homogeneous ground state of the low-temperature Sakai-Sugimoto model, JHEP 1106 (2011) 092, 1104.2291.

[170] S.-C. Zhang, A Unified Theory Based on SO(5) Symmetry of Superconductivity and Antiferromagnetism, Science 275 (5303) (1997) 1089-1096.

[171] D. Vollhardt and P. Wolfle, The Superfluid Phases of Helium 3. Taylor and Francis, London, 1990.

[172] P. Goswami and B. Roy, Axionic superconductivity in three dimensional doped narrow gap semiconductors, 1307.3240 .

[173] A. F. A. W. W. L. A. P. Schnyder, S. Ryu, Classification of topological insulators and superconductors in three spatial dimensions, Phys. Rev. B 78 (2008) 195125.

[174] L. A. Pando Zayas and D. Reichmann, A Holographic Chiral $p_{x}+i p_{y}$ Superconductor, Phys.Rev. D85 (2012) 106012, 1108.4022.

[175] W.-Y. Wen, M.-S. Wu, and S.-Y. Wu, A Holographic Model of Two-Band Superconductor, Phys.Rev. D89 (2014) 066005, 1309.0488.

[176] Z.-Y. Nie, R.-G. Cai, X. Gao, and H. Zeng, Competition between the s-wave and p-wave superconductivity phases in a holographic model, JHEP 1311 (2013) 087, 1309.2204.

[177] A. Amoretti, A. Braggio, N. Maggiore, N. Magnoli, and D. Musso, Coexistence of two vector order parameters: a holographic model for ferromagnetic superconductivity, JHEP 1401 (2014) 054, 1309.5093.

[178] A. Donos, J. P. Gauntlett, and C. Pantelidou, Competing p-wave orders, Class.Quant.Grav. 31 (2014) 055007, 1310.5741.

[179] A. S. Y.-i. Shin, C. H. Schunck and W. Ketterle, Phase diagram of a two-component Fermi gas with resonant interactions, Nature 451 (2008) 689693. 
[180] L.-y. He, M. Jin, and P.-f. Zhuang, Pion superfluidity and meson properties at finite isospin density, Phys.Rev. D71 (2005) 116001, hep-ph/0503272.

[181] M. Chernodub and A. Nedelin, Phase diagram of chirally imbalanced QCD matter, Phys.Rev. D83 (2011) 105008, 1102.0188.

[182] R. Combescot, Introduction to FFLO phases and collective mode in the BEC-BCS crossover, 0702399v1.

[183] P. Fulde and R. A. Ferrell, Superconductivity in a Strong Spin-Exchange Field, Phys. Rev. 135 (1964) A550.

[184] A. larkin and Y. Ovchinnikov, Nonuniform state of superconductors, Zh.Eksp.Teor.Fiz. 47 (1964) 1136-1146.

[185] A. I. Larkin and Y. N. Ovchinnikov, Inhomogeneous state of superconductors, Sov. Phys. JETP 20 (1965) 762.

[186] J. Erdmenger, V. Grass, P. Kerner, and T. H. Ngo, Holographic Superfluidity in Imbalanced Mixtures, JHEP 1108 (2011) 037, 1103.4145.

[187] M. Ammon, J. Erdmenger, M. Kaminski, and P. Kerner, Superconductivity from gauge/gravity duality with flavor, Phys.Lett. B680 (2009) 516-520, 0810.2316.

[188] A. Krikun, V. Kirilin, and A. Sadofyev, Holographic model of the $S^{ \pm}$multiband superconductor, JHEP 1307 (2013) 136, 1210.6074.

[189] M. Ammon, J. Erdmenger, V. Grass, P. Kerner, and A. O'Bannon, On Holographic p-wave Superfluids with Back-reaction, Phys.Lett. B686 (2010) 192-198, 0912.3515.

[190] R. E. Arias and I. S. Landea, Backreacting p-wave Superconductors, JHEP 1301 (2013) 157, 1210.6823.

[191] S. Uchino, M. Kobayashi, and M. Ueda, Bogoliubov Theory and Lee-Huang-Yang Correction in Spin-1 and Spin-2 Bose-Einstein Condensates in the Presence of the Quadratic Zeeman Effect, Phys.Rev. A81 (2010) 063632, 0912.0355].

[192] L. Landau and E. Lifshitz, Course of Theoretical Physcis, Vol. 9, Statistical Physics. Pergamon Press.

[193] C. Hoyos-Badajoz, K. Landsteiner, and S. Montero, Holographic meson melting, JHEP 0704 (2007) 031, hep-th/0612169.

[194] M. Kaminski, K. Landsteiner, F. Pena-Benitez, J. Erdmenger, C. Greubel, et. al., Quasinormal modes of massive charged flavor branes, JHEP 1003 (2010) 117, 0911.3544 .

[195] J. P. Gauntlett, J. Sonner, and T. Wiseman, Holographic superconductivity in M-Theory, Phys.Rev.Lett. 103 (2009) 151601, 0907.3796.

[196] S. S. Gubser, C. P. Herzog, S. S. Pufu, and T. Tesileanu, Superconductors from Superstrings, Phys.Rev.Lett. 103 (2009) 141601, [0907.3510].

[197] N. Bobev, N. Halmagyi, K. Pilch, and N. P. Warner, Supergravity Instabilities of Non-Supersymmetric Quantum Critical Points, Class.Quant.Grav. 27 (2010) 235013, 1006.2546. 
[198] N. Bobev, A. Kundu, K. Pilch, and N. P. Warner, Minimal Holographic Superconductors from Maximal Supergravity, JHEP 1203 (2012) 064, 1110.3454.

[199] M. Lublinsky and I. Zahed, Anomalous Chiral Superfluidity, Phys.Lett. B684 (2010) 119-122, 0910.1373.

[200] S. Lin, An anomalous hydrodynamics for chiral superfluid, Phys.Rev. D85 (2012) 045015, 1112.3215.

[201] S. Lin, On the anomalous superfluid hydrodynamics, 1104.5245.

[202] J. Bhattacharya, S. Bhattacharyya, S. Minwalla, and A. Yarom, A Theory of first order dissipative superfluid dynamics, 1105.3733 .

[203] S. Chapman, C. Hoyos, and Y. Oz, Superfluid Kubo Formulas from Partition Function, 1310.2247 .

[204] G. M. Newman and D. T. Son, Response of strongly-interacting matter to magnetic field: Some exact results, Phys. Rev. D73 (2006) 045006, hep-ph/0510049.

[205] V. A. Rubakov, On chiral magnetic effect and holography, 1005.1888.

$[206]$ 